\title{
A SEARCH FOR THE HIGGS BOSON IN THE ZH CHANNEL WITH THE D-ZERO DETECTOR AT THE FERMILAB TEVATRON COLLIDER
}

\author{
BY \\ JAMES MATTHEW HEINMILLER \\ B.S. (University of Illinois at Chicago) 1999 \\ M.S. (University of Illinois at Chicago) 2002
}

\section{THESIS}

Submitted in partial fulfillment of the requirements for the degree of Doctor of Philosophy in Physics in the Graduate College of the University of Illinois at Chicago, 2006

Chicago, Illinois 
To Roberta, Kyle, and Aaron 


\section{TABLE OF CONTENTS}

CHAPTER

PAGE

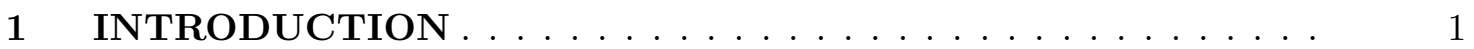

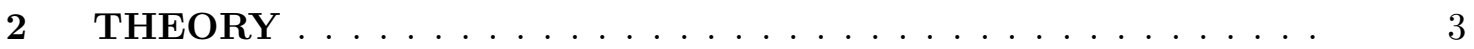

2.1 The Standard Model . . . . . . . . . . . . . . . . 4

2.1.1 Quarks and Leptons . . . . . . . . . . . . . . 4

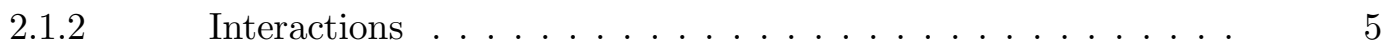

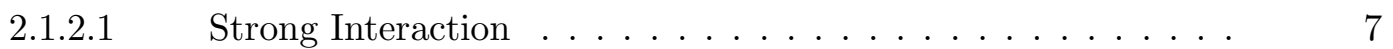

2.1.2.2 Electroweak Interaction $\ldots \ldots \ldots \ldots$

2.1.3 The Higgs Mechanism . . . . . . . . . . . . . . . . . . . 10

2.1.4 Constraints on the Higgs Mass . . . . . . . . . . . . . 12

$2.2 \quad$ Search for the Higgs at the Tevatron . . . . . . . . . . . 15

2.2.1 Higgs Sensitivity Studies . . . . . . . . . . . . . 16

$2.2 .2 \quad$ Overview of the ZH Search . . . . . . . . . . . . 17

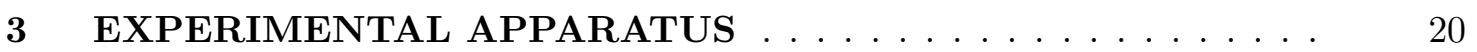

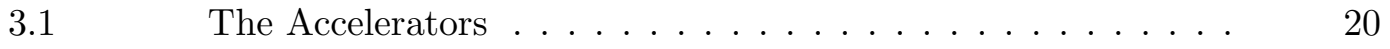

3.1.1 The Cockcroft Walton . . . . . . . . . . . . 20

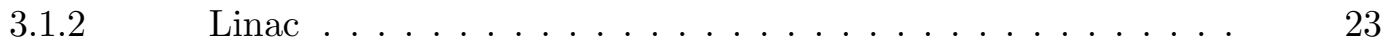

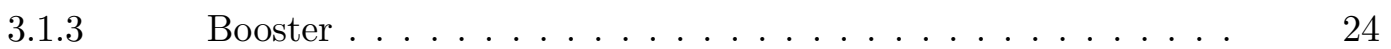

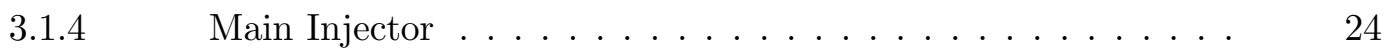

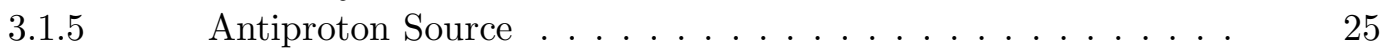

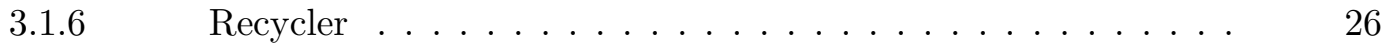

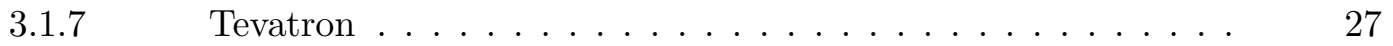

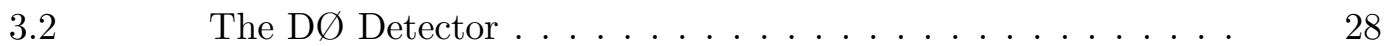

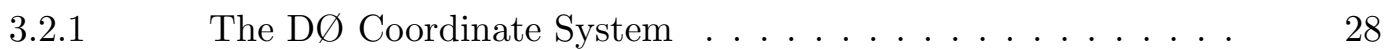

3.2.2 Luminosity Monitor . . . . . . . . . . . . . . . . . . 32

3.2.3 The Central Tracking System . . . . . . . . . . . . . 36

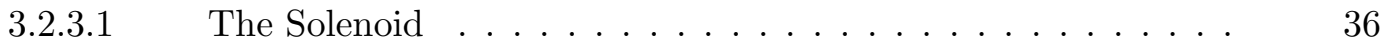

3.2.3.2 The Silicon Microstrip Tracker Detector . . . . . . . . . . . 37

3.2.3.3 The Central Fiber Tracker Detector . . . . . . . . . . . . 42

3.2.4 The Preshower Detectors . . . . . . . . . . . . . 45

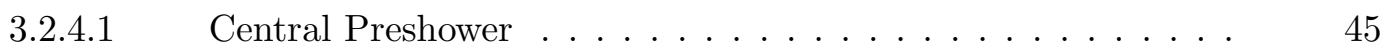

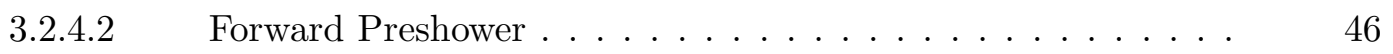

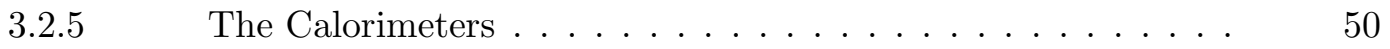

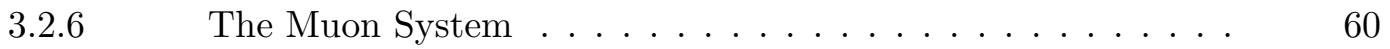

4 THE DØ TRIGGER AND DATA ACQUISITION SYSTEMS . 65 


\section{TABLE OF CONTENTS (Continued)}

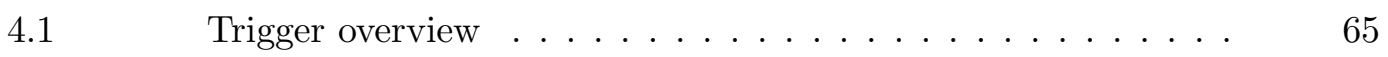

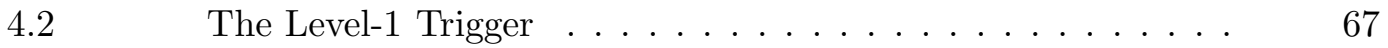

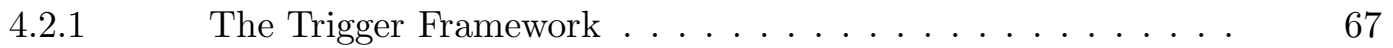

4.2.2 The Level-1 Calorimeter Trigger . . . . . . . . . . . . 67

4.2.3 The Level-1 Central Track Trigger . . . . . . . . . . . . 68

4.2.4 The Level-1 Muon Trigger . . . . . . . . . . . . . . . . 68

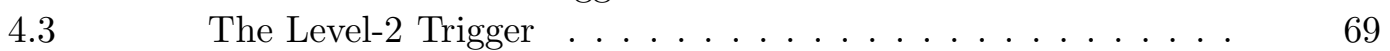

4.3.1 Level-2 Global Processor . . . . . . . . . . . . . 69

4.3.2 Level-2 Calorimeter Preprocessor . . . . . . . . . . . . 70

4.3.3 Level-2 Silicon Track Trigger Preprocessors . . . . . . . . . . 71

4.3.4 Level-2 Central Track Trigger Preprocessor . . . . . . . . . . 71

4.3.5 Level-2 Preshower Preprocessor . . . . . . . . . . . . . . . . . 72

4.3.6 Level-2 Muon Preprocessor . . . . . . . . . . . . . . . . . . 72

$4.4 \quad$ Level-3 Trigger and Data Acquisition . . . . . . . . . . 72

5 OFFLINE EVENT RECONSTRUCTION AND OBJECT IDEN-

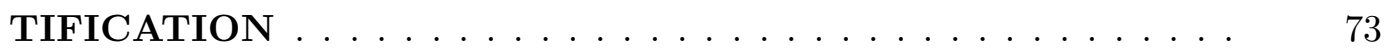

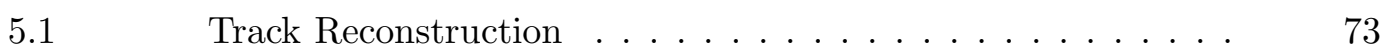

$5.2 \quad$ Primary and Secondary Vertex Reconstruction . . . . . . . . 76

$5.3 \quad$ Electromagnetic Object Reconstruction . . . . . . . . . . . 77

$5.4 \quad$ Jet Reconstruction and Identification . . . . . . . . . . . 80

5.4.1 The Jet Reconstruction Algorithm . . . . . . . . . . . 80

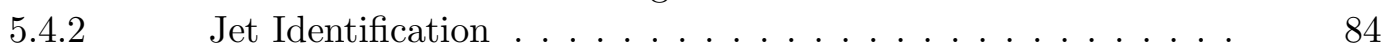

$5.4 .3 \quad$ Jet Energy Scale . . . . . . . . . . . . . . . . . . . . 85

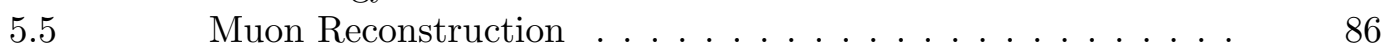

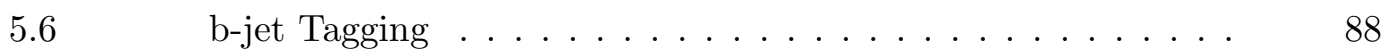

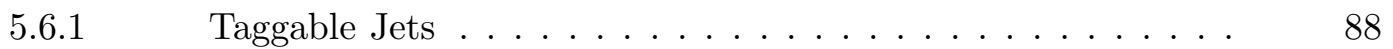

5.6.1.1 Jet LIfetime Probability (JLIP) $\ldots \ldots \ldots \ldots \ldots \ldots$

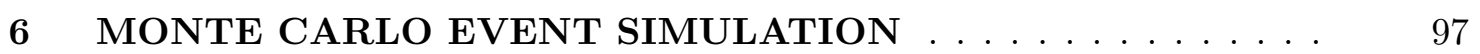

6.1 The PYTHIA Event Generator . . . . . . . . . . . . . . 97

6.2 The ALPGEN Event Generator . . . . . . . . . . . . . . . 99

$6.3 \quad$ MCFM Simulation . . . . . . . . . . . . . . 99

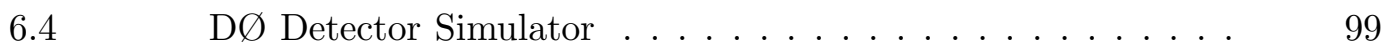

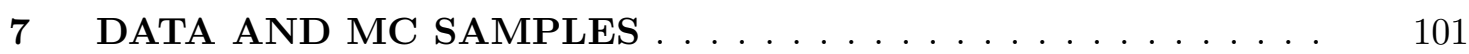

$7.1 \quad$ Data Sample. . . . . . . . . . . . . . . . . . 101

7.2 Monte Carlo Samples . . . . . . . . . . . . . . 105

$7.3 \quad$ Event Selection . . . . . . . . . . . . . . . . . 110

7.3.1 Primary Vertex . . . . . . . . . . . . . . . . 110

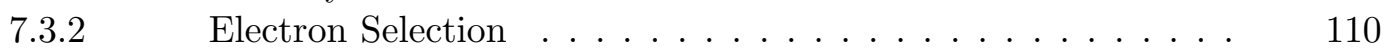

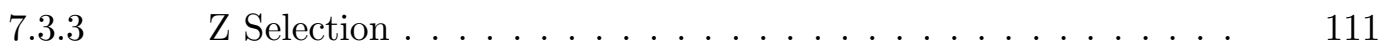




\section{TABLE OF CONTENTS (Continued)}

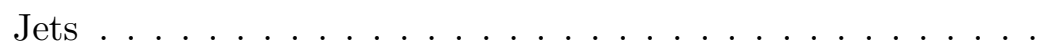

8 OBJECT RECONSTRUCTION AND SELECTION EFFICIENCIES

8.2 Electron Reconstruction and Identification Efficiency . . . . . 130

$8.3 \quad$ Electron-Track Match Efficiency . . . . . . . . . . . . . 137

$8.4 \quad$ Jet Reconstruction and Identification Efficiency . . . . . . . . 143

8.4.1 "Z $p_{T}$ balance" scale factor . . . . . . . . . . . . . 143

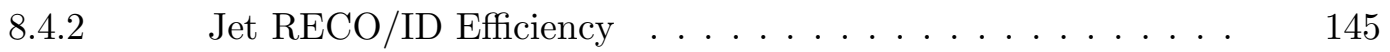

8.5 Jet Taggability Efficiencies . . . . . . . . . . . . . . . . . . 155

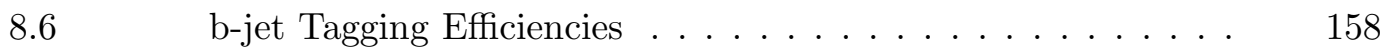

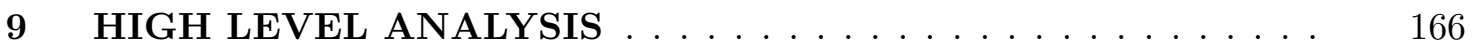

$9.1 \quad$ High Level Analysis Cuts . . . . . . . . . . . . . . . 166

$9.2 \quad$ Background Processes to the Higgs Signal . . . . . . . . . . . . . 167

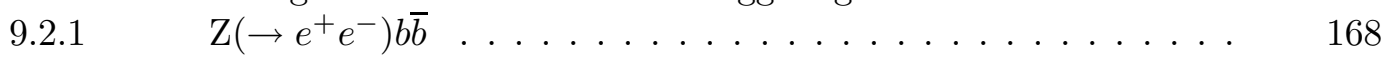

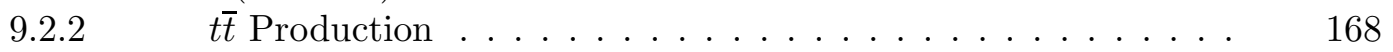

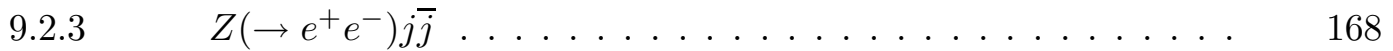

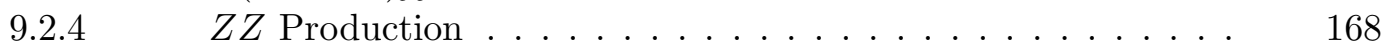

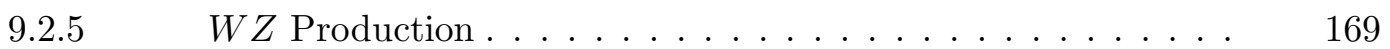

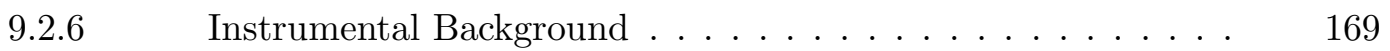

$9.3 \quad$ Data and MC Comparisons . . . . . . . . . . . . . . 174

$9.4 \quad$ Higgs Mass Search Windows . . . . . . . . . . . . . . . . . . . 182

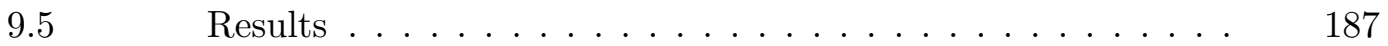

10 ZH CROSS SECTION LIMITS . . . . . . . . . . . . . . . 195

$10.1 \quad$ Background Uncertainties . . . . . . . . . . . . . . 195

10.1.1 Scale Factor Uncertainties . . . . . . . . . . . . . . 196

10.1.1.1 Trigger Efficiency Uncertainty . . . . . . . . . . . . . . . 196

10.1.1.2 Electron RECO/ID Scale Factor Uncertainty . . . . . . . . . . 196

10.1.1.3 Track Match Scale Factor Uncertainty . . . . . . . . . . . . . . 196

10.1.1.4 Jet RECO/ID Scale Factor Uncertainty . . . . . . . . . . . . . . 196

10.1.1.5 Jet Taggability Scale Factor Uncertainty . . . . . . . . . . . . . . 196

10.1.1.6 JLIP Scale Factor Uncertainty . . . . . . . . . . . . . . . . . . . 197

10.1.2 Energy Uncertainty . . . . . . . . . . . . . . . . . . . . 197

10.1.2.1 Jet Energy Scale Uncertainty . . . . . . . . . . . . . . . . . . . 197

10.1.2.2 Electron Energy Smear Uncertainty . . . . . . . . . . . . . . . . 197

10.1.2.3 Jet Energy Smear Uncertainty . . . . . . . . . . . . . . . . . . . . 197

10.1.3 Overall Experimental Uncertainties . . . . . . . . . . . . 197 


\section{TABLE OF CONTENTS (Continued)}

10.1.4 Physics Background Uncertainty . . . . . . . . . . . . . . . 198

10.1.4 $t \bar{t} \ldots \ldots \ldots \ldots \ldots \ldots \ldots \ldots \ldots$

10.1.4.2 ZZ and $\mathrm{WZ} \ldots \ldots \ldots \ldots \ldots \ldots \ldots$

$10.1 .4 .3 \quad \mathrm{Zjj} \ldots \ldots \ldots \ldots \ldots \ldots \ldots \ldots$

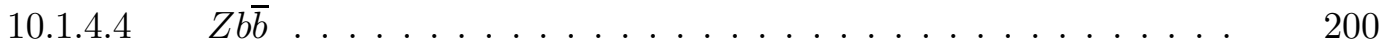

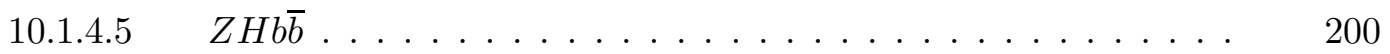

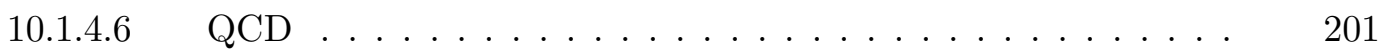

10.1.5 Statistical Uncertainty of the MC samples . . . . . . . . . . . . 201

10.1.6 Overall Uncertainties . . . . . . . . . . . . . . . . 201

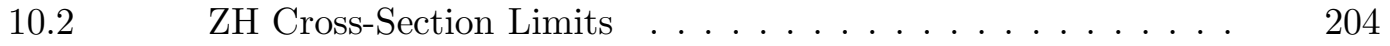

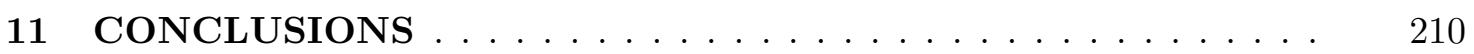

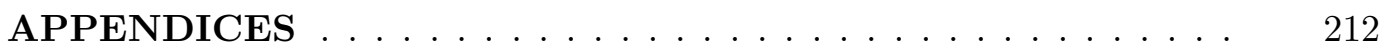

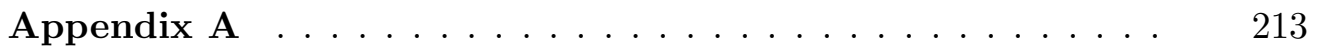

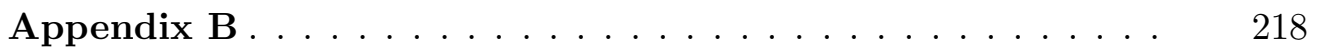

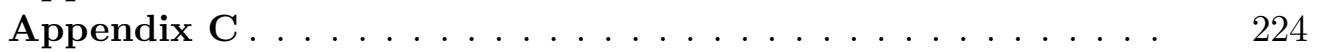

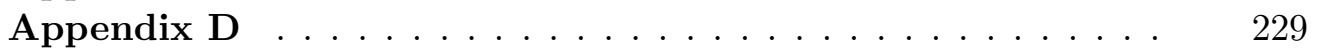

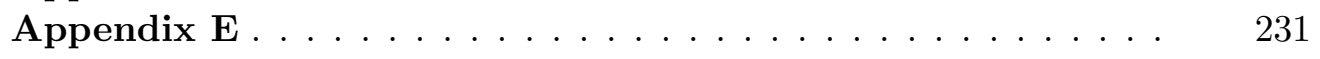

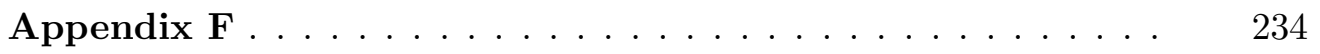

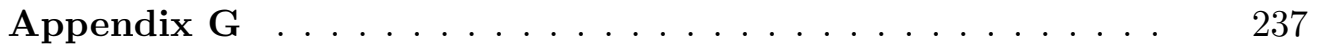

CITED LITERATURE . . . . . . . . . . . . . . . 243 


\section{LIST OF TABLES}

\section{TABLE}

PAGE

I FUNDAMENTAL FERMION BUILDING BLOCKS OF MATTER. THE FORCES ARE MEDIATED BY THE PHOTON (P), WEAK BOSONS $(\mathrm{W})$, AND GLUONS $(\mathrm{G}) \ldots \ldots \ldots \ldots \ldots$

II $\quad$ FUNDAMENTAL INTERACTIONS OF NATURE IN THE SM. . 6

III LOCATION OF SILICON MICROSTRIP TRACKER COMPONENTS ALONG THE Z DIRECTION STARTING FROM THE CENTER OF THE DETECTOR. . . . . . . . . . . . . . . . 40

IV SILICON MICROSTRIP TRACKER DETECTOR COMPONENT RADII (MEASURED FROM THE CENTER OF THE BEAM PIPE). 41

V CENTRAL CALORIMETER PARAMETERS . . . . . . . . . 52

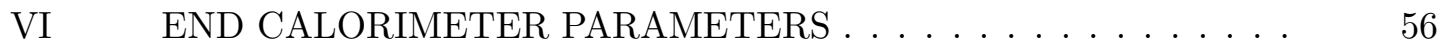

VII TRACK CATEGORIES USED FOR THE PARAMETERIZATION OF THE IMPACT PARAMETER RESOLUTION FUNCTIONS. . 90

VIII TRIGGERS USED IN THE ANALYSIS. . . . . . . . . . . . . . 103

IX THE TRIGGER NAMES BROKEN UP INTO THEIR CORRESPONDING L1, L2, AND L3 TRIGGER TERMS. . . . . . . . . . . 103

X L1, L2, AND L3 TRIGGERS USED IN THIS ANALYSIS. . . . . . 104

XI SUMMARY OF MONTE CARLO SAMPLES USED IN THIS ANAL-

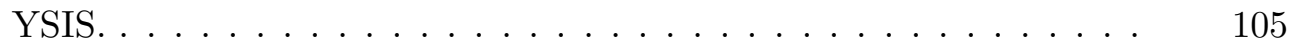

XII JET RESOLUTION PARAMETERS IN DATA AND MC. . . . . . 107

XIII AVERAGE TRIGGER EFFICIENCIES (\%) WITH RESPECT TO TRIGGER VERSION AND JET MULTIPLICITY. THE UNCERTAINTIES GIVEN ARE STATISTICAL. . . . . . . . . . . 128 


\section{LIST OF TABLES (Continued)}

$\underline{\text { TABLE }}$

PAGE

XIV BACKGROUND SUBTRACTED ELECTRON RECO/ID EFFICIENCIES VS JET MULTIPLICITY FOR DATA AND MC. THE UNCERTAINTIES ARE STATISTICAL. . . . . . . . . . . . 135

XV ELECTRON RECO/ID UNCERTAINTIES. . . . . . . . . . 136

XVI BACKGROUND SUBTRACTED ELECTRON-TRACK MATCH EFFICIENCIES VS JET MULTIPLICITY FOR DATA AND MC. THE UNCERTAINTIES ARE STATISTICAL. . . . . . . . . . . 141

XVII ELECTRON-TRACK MATCH UNCERTAINTIES. . . . . . . . . . . 142

XVIII THE MC AND "DATA" JET RECO/ID EFFICIENCY AS A FUNCTION OF THE PARTICLE JET $P_{T}$ SMEARED WITH THE JET ENERGY RESOLUTIONS FROM DATA. . . . . . . . . . . . .

XIX THE MC AND "DATA" JET RECO/ID EFFICIENCY ERRORS AS A FUNCTION OF THE PARTICLE JET $P_{T}$ SMEARED WITH THE JET ENERGY RESOLUTIONS FROM DATA .................

XX SUMMARY OF THE DATA, SIGNAL, AND BACKGROUND PROCESSES. THE UNCERTAINTIES INCLUDE THE STATISTICAL AND SYSTEMATIC EFFECTS. . . . . . . . . . . . . . . .

XXI INVARIANT MASS RESOLUTIONS OBTAINED BY A GAUSSIAN FIT. THE AVERAGE RELATIVE RESOLUTION IS FOUND TO BE 18\%. THE HIGGS SEARCH WINDOWS ARE CENTERED ON THE MEAN AND SPAN $\pm 1.5 \sigma . \ldots \ldots \ldots \ldots$

XXIII SIGNIFICANCE STUDY, NUMBER OF ZH EVENTS DIVIDED BY THE SQUARE ROOT OF THE NUMBER OF EVENTS FROM ALL OF THE BACKGROUND PROCESSES FOR 1 B TAG AND 2 B TAGS USING THE SIX CERTIFIED JLIP CUTS. . . . . . . .

XXIV THE EFFICIENCIES OF THE FIVE MC SIGNAL SAMPLES (IN

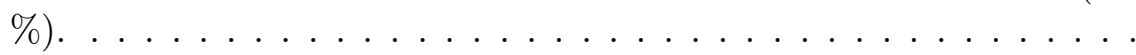

XXV THE EFFICIENCIES OF THE FIVE MC SIGNAL SAMPLES AND THE MC BACKGROUNDS (IN \%). THE $Z H$ SAMPLE IS FOR A HIGGS MASS OF 115 GEV. 


\section{LIST OF TABLES (Continued)}

XXVI NUMBER OF EVENTS IN DATA WITH THE SM MC BACKGROUND PROCESSES AT FOUR DIFFERENT SELECTION CUTS. THE $Z H$ SAMPLE IS FOR A HIGGS MASS OF 115 GEV. THE $\pm 1 \sigma$ UNCERTAINTIES ARE STATISTICAL AND SYSTEMATIC.

XXVII NUMBER OF EVENTS WITH EXCLUSIVE SINGLE B TAGGING. EXPECTED NUMBER OF ZH EVENTS AND BACKGROUND CONTRIBUTIONS WITHIN $M_{H}=105,115,125,135$, AND 145 GEV SEARCH WINDOWS. THE $\pm 1 \sigma$ UNCERTAINTIES ARE STATISTICAL AND SYSTEMATIC. . . . . . . . . . . . . .

XXVIII NUMBER OF EVENTS WITH DOUBLE B TAGGING. EXPECTED NUMBER OF ZH EVENTS AND BACKGROUND CONTRIBUTIONS WITHIN $M_{H}=105,115,125,135$, AND 145 GEV SEARCH WINDOWS. THE $\pm 1 \sigma$ ERRORS ARE STATISTICAL AND SYSTEMATIC. . . . . . . . . . . . . . . . .

XXIX A SUMMARY OF THE SOURCES OF EXPERIMENTAL UNCERTAINTY IN THE NUMBER OF EVENTS AFTER THE FINAL SELECTION CUT. . . . . . . . . . . . . . . 198

XXX MONTE CARLO CROSS SECTION UNCERTAINTIES. . . . . . . 199

XXXI THE RELATIVE STATISTICAL UNCERTAINTIES FOR THE MC SAMPLES $\ldots \ldots \cdot \cdots \cdot \cdots \cdot \cdots \cdot \cdots \cdot \cdots \cdot \cdots \cdot \cdots$

XXXII OVERALL UNCERTAINTIES FOR THE SIGNAL $\left(Z H_{115}\right)$ AND THE BACKGROUND PROCESSES. . . . . . . . . . . . . .

XXXIII THE EFFICIENCIES OF THE FIVE MC SIGNAL SAMPLES (IN

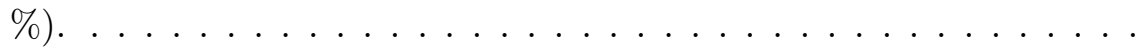

XXXV SUMMARY OF THE ZH ANALYSIS WITH EXCLUSIVE SINGLE B TAGGING. EXPECTED NUMBER OF ZH EVENTS AND BACKGROUND CONTRIBUTIONS FOR $M_{H}=105,115,125,135$, AND 145 GEV, ALONG WITH 95\% CL CROSS-SECTION UPPER

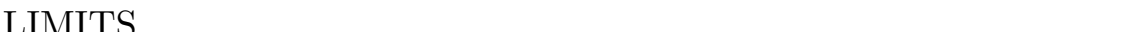

XXXVII SUMMARY OF THE ZH ANALYSIS WITH DOUBLE B TAGGING. EXPECTED NUMBER OF ZH EVENTS AND BACKGROUND CONTRIBUTIONS FOR $M_{H}=105,115,125,135$, AND $145 \mathrm{GEV}$, ALONG WITH 95\% CL CROSS-SECTION UPPER LIMITS. . . . 


\section{LIST OF TABLES (Continued)}

TABLE

PAGE

XXXVIIIEM OUTPUT TO L2GLOBAL. . . . . . . . . . . . .

225

XXXIX EM OUTPUT TO L2GLOBAL FOR TRIGGER LISTS GREATER

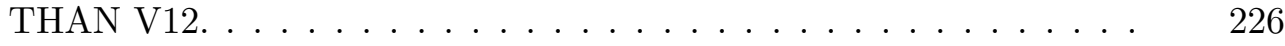

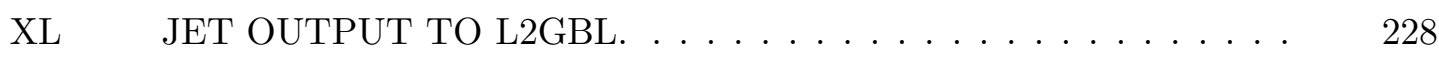




\section{LIST OF FIGURES}

1 Limits on the Higgs boson mass from electroweak measurements. The figure on the left shows the relationship between the $\mathrm{M}_{H}$ to the mass of the $W^{ \pm}$and the top quark. The figure on the right uses high precision electroweak data to constrain the mass of the Higgs. The $M_{H}$ is constrained to be greater than $114.4 \mathrm{GeV}$ from LEP experiments (shown in

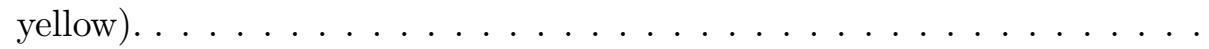

2 Production cross section of the Higgs boson (left plot) at the Tevatron and the Higgs boson branching ratio (right plot). . . . . . . . .

3 Projected Tevatron limits on the SM Higgs boson mass (in GeV) as a function of integrated luminosity per experiment with 95\% CL exclusion, $3 \sigma$ evidence, and $5 \sigma$ discovery. . . . . . . . . . . . .

4 A Feynman diagram or the $\mathrm{Z}\left(\rightarrow e^{+} e^{-}\right) \mathrm{H}(\rightarrow b \bar{b})$ channel. $\ldots \ldots \ldots$

$5 \quad$ Feynman diagrams of physics processes that contribute to the background.

6 The schematic view of the accelerator chain at Fermilab. The Recycler is housed above the Main Injector. The Debuncher and Accumulator are in the Antiproton Source ring. . . . . . . . . . . . .

7 Hand drawing of the $\mathrm{H}^{-}$ion source or magnetron. . . . . . . .

8 A drawing of the Linac RF cavity. . . . . . . . . . .

9 A schematic of the antiproton generating nickel target. . . . . . . .

10 A diagram showing the nickel target and lithium lens. . . . . . . .

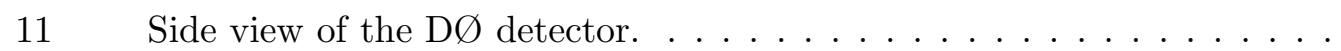

12 The $\mathrm{D} \varnothing$ detector coordinate system. . . . . . . . . . .

13 A diagram of the D $\varnothing$ luminosity monitors. The $\mathrm{r}-\phi$ view is shown on the left, while the $\mathrm{r}-\mathrm{z}$ view of the two monitors is shown on the right. The photomultiplier tubes are represented by small circles on each tile. 


\section{LIST OF FIGURES (Continued)}

FIGURE

PAGE

14 The $\mathrm{z}$ vertex distribution from inelastic $p \bar{p}$ collisions. . . . . . . . .

15 The total amount of luminosity delivered to and recorded by the $\mathrm{D} \varnothing$

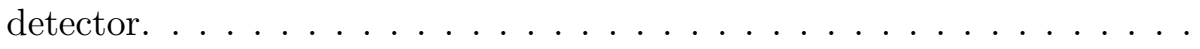

16 Side view of the Central Tracking detectors. . . . . . . . . . .

17 The magnetic field lines extended out to the magnetic toroid. The strength of the magnetic field is given in kiloGauss $(10 \mathrm{kG}=1 \mathrm{~T}) \ldots$

18 A 3D layout of the Silicon Mircostrip Tracker. . . . . . . . . . . .

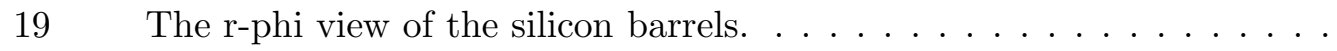

20 The r-phi view of the silicon wedge detectors of the F-disks. . . . . . . .

21 a) The Central Fiber Tracker located between the Silicon Microstrip Tracker and the Solenoid. b) The cross section blow up of the fibers from two layers. . . . . . . . . . . . . . . . . . .

22 a) The r-z diagram of the Central Fiber Tracker. b) A cross section of view of the fibers. . . . . . . . . . . . . . .

23 Schematic r-z side view of the central preshower detector. . . . . . . .

24 Schematic r-phi view of the central preshower detector. . . . . . . . .

25 The cross section of the central and forward preshower scintillating triangular strips. . . . . . . . . . . . . . . .

26 The layout of three layers of scintillating strips - one axial and two at stereo angles. . . . . . . . . . . . . . . . .

27 The forward preshower detectors attached to each of the calorimeter end

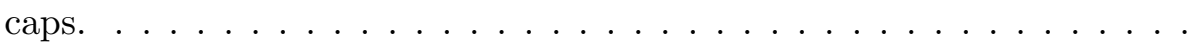

28 A view of the central and end cap calorimeters. . . . . . . . . .

29 Schematic view of a unit cell in the calorimeter. . . . . . . . . . . . .

30 One quarter view of the calorimeter showing the segmented readout. . . 


\section{LIST OF FIGURES (Continued)}

FIGURE

PAGE

31 The calorimeter readout towers in $\eta$ versus the layers distributed along the shower development. . . . . . . . . . . .

32 The shape of the signals detected and processed through the electronics in the calorimeter. . . . . . . . . . . . . . . . . . . .

33 The electronic readout of the unit cell to the analog digital converter. .

34 Cross section through proportional drift chambers. . . . . . . . .

$35 \quad$ A cross section of the mini-drift tubes. . . . . . . . . .

36 Simulated muon trajectory through the $\mathrm{D} \varnothing$ detector. . . . . . . .

37 The flow of the data from the $\varnothing \varnothing$ detector to storage onto magnetic tapes.

38 The flow of data from the L1 inputs to the Level-2 trigger. . . . . . . . .

39 The decay path of quarks and gluons. Quarks and gluons after final state radiation form parton jets. Hadrons and leptons after hadronization form particle jets. Energy deposits in the calorimeter form calorimeter

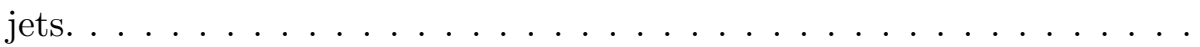

40 The jet energy scale corrections, left, and uncertainties, right, for reconstructed calorimeter jets measured in data. . . . . . . . . . . . . .

41 Impact parameter significance $\frac{I P}{\sigma}$ for tracks measured in data using four different track selections based on the number of hits in the SMT and CFT detectors. The peak is fitted with a Gaussian function while the tails are fitted with an exponential function. . . . . . . . . . .

42 Resolution $\mathrm{R}(\mathrm{s})$ distributions using the negative side of the IP significance curves. There are 29 resolution fits. Four of the fits are shown above. The resolution distributions are fit by adding four Gaussian func-

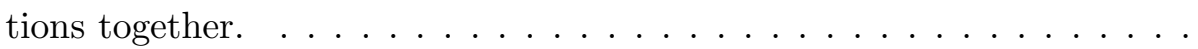

43 Using the resolution functions from the previous figure, a track probability is calculated using only positive sign IP significance tracks. . . . . 


\section{LIST OF FIGURES (Continued)}

\section{FIGURE}

44 Combining all the positive sign IP significance tracks generate the JLIP probability. The upper left plot shows that using the negative signed $S_{I P}$ tracks gives a flat distribution for a b-tagged enhanced data sample. The upper right plot shows a flat probability distribution for light jets from $\mathrm{MC}$. The bottom right plot shows that $\mathrm{b}$ jets in $\mathrm{MC}$ are highly peaked at low JLIP probabilities. . . . . . . . . . . . . . . . . . .

45 Jet $p_{T}$ resolutions for different $\eta_{\text {det }}$ regions in data. The points below $\sim 50 \mathrm{GeV}$ are obtained using $\gamma+$ jet events, whereas for $p_{T} \geq 50 \mathrm{GeV}$ resolutions are measured using di-jet events. Bands of $\pm 1 \sigma$ statistical error are also shown. . . . . . . . . . . . . . . . . .

46 Jet $p_{T}$ resolutions for different $\eta_{\text {det }}$ regions in MC. The points below $\sim 50 \mathrm{GeV}$ are obtained using $\gamma+$ jet events, whereas for $p_{T} \geq 50 \mathrm{GeV}$ resolutions are measured using di-jet events. Bands of $\pm 1 \sigma$ statistical error are also shown. . . . . . . . . . . . . . . . .

47 The primary vertex after the di-em invariant mass cut of $75 \mathrm{GeV}<$ $M_{e e}<105 \mathrm{GeV}$ has been applied. The MC sample is normalized to the number of events in data. . . . . . . . . . . . . . . . . . . . .

48 The $\eta$ distribution of the two electrons from the $Z$ boson after the di-em invariant mass cut of $75 \mathrm{GeV}<M_{e e}<105 \mathrm{GeV}$ has been applied. The MC sample is normalized to the number of events in data. . . . . . . .

The $\phi$ distribution of the two electrons from the $Z$ boson after the di-em invariant mass cut of $75 \mathrm{GeV}<M_{e e}<105 \mathrm{GeV}$ has been applied. The MC sample is normalized to the number of events in data. . . . . . . . .

50 The $p_{T}$ distribution of the two electrons from the $Z$ boson after the diem invariant mass cut of $75 \mathrm{GeV}<M_{e e}<105 \mathrm{GeV}$ has been applied. The MC sample is normalized to the number of events in data. . . . . .

51 The $\Delta \phi$ distribution between the two electrons from the $Z$ boson after the di-em invariant mass cut of $75 \mathrm{GeV}<M_{e e}<105 \mathrm{GeV}$ has been applied. The MC sample is normalized to the number of events in data.

52 The $\Delta R$ distribution between the two electrons from the $Z$ boson after the di-em invariant mass cut of $75 \mathrm{GeV}<M_{e e}<105 \mathrm{GeV}$ has been applied. The MC sample is normalized to the number of events in data. 


\section{LIST OF FIGURES (Continued)}

FIGURE

PAGE

53 The di-em invariant mass distribution using two electron objects with track matches. PYTHIA is normalized to have the same number of events in the window of $80 \mathrm{GeV}<\mathrm{M}_{e e}<100 \mathrm{GeV}$ as data. . . . . . . . .

54 The di-em invariant mass distribution using an electron with a track match and an opposite sign track. The momentum vectors of the opposite sign tracks in the MC are smeared to have the same momentum resolution as the probe tracks in data. PYTHIA is normalized to have the same number of events in the window of $75 \mathrm{GeV}<\mathrm{M}_{e e}<105 \mathrm{GeV}$ as data. . . . . . . . . . . . . . . . .

$55 \quad Z p_{T}$ distribution for the $Z$ inclusive sample after the di-em invariant mass cut of $75 \mathrm{GeV}<M_{e e}<105 \mathrm{GeV}$ has been applied. The PyтнiA MC sample is normalized to the number of events in data. . . . . . . .

56 Jet $p_{T}$ distribution in the $Z$ inclusive sample after the di-em invariant mass cut of $75 \mathrm{GeV}<M_{e e}<105 \mathrm{GeV}$ has been applied. The PYTHIA MC sample is normalized to the number of events in data. . . . . . . .

57 Jet $\eta$ distribution for the $Z$ inclusive sample after the di-em invariant mass cut of $75 \mathrm{GeV}<M_{e e}<105 \mathrm{GeV}$ has been applied. The PYTHIA MC sample is normalized to the number of events in data. . . . . . . . .

58 Jet $\phi$ distribution for the $Z$ inclusive sample after the di-em invariant mass cut of $75 \mathrm{GeV}<M_{e e}<105 \mathrm{GeV}$ has been applied. The PYTHIA MC sample is normalized to the number of events in data. . . . . . . . .

59 Taggable jet $p_{T}$ distributions for events that have a $\mathrm{Z}$ boson and at least one calorimeter jet. The ALPGEN+PYTHIA sample is normalized to the number of events in data. . . . . . . . . . . . . . . . . . . .

60 Taggable jet $\eta$ distributions for events that have a $\mathrm{Z}$ boson and at least one calorimeter jet. The ALPGEN+PYTHIA sample is normalized to the number of events in data. . . . . . . . . . . . . .

61 Taggable jet $\phi$ distributions for events that have a $\mathrm{Z}$ boson and at least one calorimeter jet. The ALPGEN+PYTHIA sample is normalized to the number of events in data. . . . . . . . . . . . . . .

62 Trigger efficiencies vs electron $p_{T}$ with zero or more jets in each event for different trigger list versions. . . . . . . . . . . . . 


\section{LIST OF FIGURES (Continued)}

FIGURE

PAGE

63 Trigger efficiencies vs electron $\eta_{\text {det }}$ with zero or more jets in each event for different trigger list versions. . . . . . . . . . . .

64 Trigger efficiencies vs electron $\phi_{\text {det }}$ with zero or more jets in each event for different trigger list versions. . . . . . . . . . . . .

65 Trigger efficiencies as a function of jet multiplicity. . . . . . . .

66 Trigger efficiencies as a function of jet multiplicity for the combined data sample. . . . . . . . . . . . . . . . . .

67 Electron RECO/ID efficiencies in data and MC from events using the $Z$ inclusive sample vs probe track $p_{T}$ without background subtraction. The MC sample is PYTHIA generated $Z \rightarrow e \bar{e}+X$. The probe track is in the central region $\left|\eta_{\text {det }}\right|<1.1$ of the calorimeter. . . . . . . . .

68 Electron RECO/ID efficiencies in data and MC from events using the $Z$ inclusive sample vs probe track $p_{T}$ without background subtraction. The MC sample is PYTHIA generated $Z \rightarrow e \bar{e}+X$. The probe track is in the forward region $1.5<\left|\eta_{\text {det }}\right|<2.5$ of the calorimeter. . . . . . .

69 Electron RECO/ID efficiencies in data and MC from events using the $Z$ inclusive sample vs probe track $\eta_{\text {det }}$ without background subtraction. The MC sample is PYTHIA generated $Z \rightarrow e \bar{e}+X \ldots \ldots \ldots$

70 Electron RECO/ID efficiencies in data and MC from events using the $Z$ inclusive sample vs probe track $\phi+2 \pi$ floating point remainder of $2 \pi / 32$ without background subtraction. The MC sample is PYTHIA generated $Z \rightarrow e \bar{e}+X \ldots \ldots \ldots \ldots \ldots \ldots \ldots \ldots$

71 Electron RECO/ID efficiency vs jet multiplicity for data and MC after background subtraction. The sidebands of the invariant mass peak are used for the background estimation. . . . . . . . . . . .

72 Electron-track match efficiencies in data and MC for the $Z$ inclusive sample vs probe electron $p_{T}$ without background subtraction. The MC sample is PYтHIA generated $Z \rightarrow e \bar{e}+X$. The probe electron is in the central region $\left|\eta_{\text {det }}\right|<1.1$ of the calorimeter. . . . . . . . . 


\section{LIST OF FIGURES (Continued)}

FIGURE

PAGE

73 Electron-track match efficiencies in data and $\mathrm{MC}$ for the $Z$ inclusive sample vs probe electron $p_{T}$ without background subtraction. The MC sample is PYTHIA generated $Z \rightarrow e \bar{e}+X$. The probe electron is in the forward region $1.5<\left|\eta_{\text {det }}\right|<2.5$ of the calorimeter. . . . . . . .

74 Electron-track match efficiencies in data and $\mathrm{MC}$ for the $Z$ inclusive sample vs probe electron $\eta_{\text {det }}$ without background subtraction. The MC sample is PYTHIA generated $Z \rightarrow e \bar{e}+X \ldots \ldots \ldots \ldots$

75 Electron-track match efficiencies vs jet multiplicity for data and MC. The background in data has been removed by using the sidebands of the invariant mass peak. . . . . . . . . . . . . . . . .

76 Scale factor (solid black line) vs $Z p_{T}$ overlaid with direct photon scale factor (in dashed line). $\pm 1 \sigma$ uncertainty (thinner blue lines) bound the

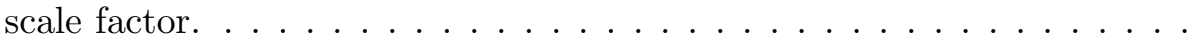

77 The efficiency to find a calorimeter jet given a particle jet in three separate MC simulations. . . . . . . . . . . . . . . . .

78 Jet RECO/ID efficiency in MC for $|\eta|<0.7$ vs smeared particle jet $p_{T}$. The three lines represent the fit to the data (central line) and the $\pm 1 \sigma$

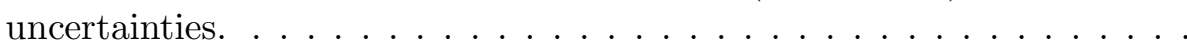

79 Jet RECO/ID efficiency in "data" for $|\eta|<0.7$ vs smeared particle jet $p_{T}$. The three lines represent the fit to the data (central line) and the $\pm 1 \sigma$ uncertainties. . . . . . . . . . . . . . .

80 Jet RECO/ID efficiency in MC for $0.7<|\eta|<1.5$ vs smeared particle jet $p_{T}$. The three lines represent the fit to the data (central line) and the $\pm 1 \sigma$ uncertainties. . . . . . . . . . . . . . . .

81 Jet RECO/ID efficiency in "data" for $0.7<|\eta|<1.5$ vs smeared particle jet $p_{T}$. The three lines represent the fit to the data (central line) and the $\pm 1 \sigma$ uncertainties. . . . . . . . . . . . . . . . .

82 Jet RECO/ID efficiency in MC for $1.5<|\eta|<2.5$ vs smeared particle jet $p_{T}$. The three lines represent the fit to the data (central line) and the $\pm 1 \sigma$ uncertainties. . . . . . . . . . . . . . . . 


\section{LIST OF FIGURES (Continued)}

FIGURE

PAGE

83 Jet RECO/ID efficiency in "data" for $1.5<|\eta|<2.5$ vs smeared particle jet $p_{T}$. The three lines represent the fit to the data (central line) and the $\pm 1 \sigma$ uncertainties. . . . . . . . . . . . . . . .

84 The data points with $\pm 1 \sigma$ uncertainty bands for the jet RECO/ID efficiency in "data" for $|\eta|<0.7$ without smearing the particle jets to the energy resolution of the calorimeter jets in data. The inverted triangle shows efficiencies derived with the photon balance technique measured in data. . . . . . . . . . . . . . . . . . . .

85 The data points with $\pm 1 \sigma$ uncertainty bands for the jet RECO/ID efficiency in "data" for $0.7<|\eta|<1.5$ without smearing the particle jets to the energy resolution of the calorimeter jets in data. The inverted triangle shows efficiencies derived with the photon balance technique measured in data. . . . . . . . . . . . . . . . . . .

86 The data points with $\pm 1 \sigma$ uncertainty bands for the jet RECO/ID efficiency in "data" for $1.5<|\eta|<2.5$ without smearing the particle jets to the energy resolution of the calorimeter jets in data. The inverted triangle shows efficiencies derived with the photon balance technique measured in data. . . . . . . . . . . . . . . . . . . . .

87 Taggable jet efficiency for data and MC for $|\eta|<2.5$ as a function of

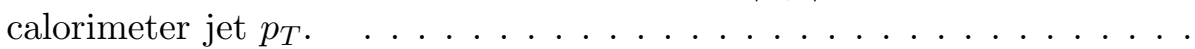

88 Taggable jet efficiency for data and MC within $|\eta|<2.5$ as a function of calorimeter jet $|\eta| . \ldots \ldots \ldots \ldots \ldots \ldots \ldots \ldots \ldots \ldots \ldots \ldots \ldots$

89 Average taggable jet efficiency of data and various MC samples. . . . .

90 Tag Rate Function $T R F_{b}$ for simulated inclusive b-tag jets, versus $E_{T}$

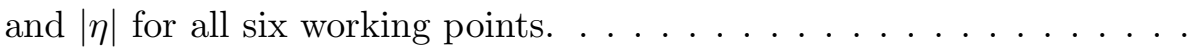

91 Tag Rate Function $T R F_{c}$ for simulated inclusive c-tag jets versus $E_{T}$ and $|\eta|$ for all six working points. . . . . . . . . . . .

92 Light quark tagging efficiency for all six working points versus jet $E_{T}$

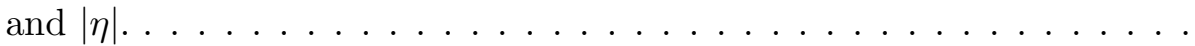

$93 \quad S F_{b}$ scale factor, ratio of the b-tagging efficiencies measured in muonin-jet data and simulation versus the jet $E_{T}$ and $|\eta|$ for all six working

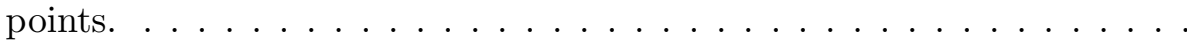




\section{LIST OF FIGURES (Continued)}

FIGURE

PAGE

94 Overall relative uncertainties on $T R F_{b}, T R F_{c}$, and $T R F_{\text {light }}$ versus jet $E_{T}$ and $|\eta|$ for all working points. The statistical uncertainties and systematics are summed in quadrature. . . . . . . . . . . .

95 JLIP efficiencies for the six working points as a function of jet $p_{T}$. The solid lines use the MC JLIP probability and then the JLIP scale factor. The square points use the JLIP TRFs for $\mathrm{b}$ jets. The MC simulation is ALPGEN+PYTHIA $Z b \bar{b} . \ldots \ldots \ldots \ldots \ldots$

96 JLIP efficiencies for the six working points as a function of $|\eta|$. The solid lines use the MC JLIP probability and then the JLIP scale factor. The square points use the JLIP TRFs for $\mathrm{b}$ jets. The MC simulation is ALPGEN+PYTHIA $Z b \bar{b}$. . . . . . . . . . . . . . . .

97 Di-electron invariant mass distribution for events with 2 or more jets in data. The fit is a convoluted Gaussian and Breit-Wigner with an exponential curve for the background. . . . . . . . . .

98 Di-electron invariant mass distribution for events with 2 or more jets in ALPGEN+PYThia MC simulated $Z j j \rightarrow e \bar{e}+j j$. The fit is a convoluted Gaussian and Breit-Wigner with an exponential curve for the background.

99 Di-electron object (inverted H-matrix 7 cuts) invariant mass distribution for events for the $Z$ inclusive sample in the QCD enhanced sample. The fit is dominated by the exponential background with a convoluted Gaussian and Breit-Wigner fit in the absence of a $Z$ boson signature. .

100 Di-electron object (inverted H-matrix 7 cuts) invariant mass distribution for 2 or more jets in the QCD enhanced sample. The fit is dominated by the exponential background with a convoluted Gaussian and BreitWigner trying to fit in the absence of a $Z$ boson signature. . . . . . . .

101 Kinematic distributions of the two electrons in the $Z+\geq 2$ jet sample: top left) $p_{T}$, top right) $\eta$, bottom left) $\phi$, bottom right) invariant mass. The simulation is normalized to the integrated luminosity of the data sample using the expected cross sections (absolute normalization). The $Z j j$ simulation is normalized so that the total number of simulated events equals the number of $Z+\geq 2$ jet events in data. . . . . . . 


\section{LIST OF FIGURES (Continued)}

FIGURE

PAGE

102 Kinematic distributions of the $Z$ boson in the $Z+\geq 2$ jet sample: top left) $p_{T}$, top right) $\Delta \phi$ of the 2 electrons, bottom left) Rapidity, bottom right) $\phi$. The simulation is normalized to the integrated luminosity of the data sample using the expected cross sections (absolute normalization). The $Z j j$ simulation is normalized so that the total number of simulated events equals the number of $Z+\geq 2$ jet events in data. . . . . . . .

103 Distributions of jets in the $Z+\geq 2$ jet sample: top left) number of jets, top right) $p_{T}$, bottom left) number of jets (log scale), bottom right) $p_{T}$ of the jets (log scale). The simulation is normalized to the integrated luminosity of the data sample using the expected cross sections (absolute normalization). The $Z j j$ simulation is normalized so that the total number of simulated events equals the number of $Z+\geq 2$ jet events in data . . . . . . . . . . . . . . . . . . . .

104 Distributions of jets in the $Z+\geq 2$ jet sample: top left) $p_{T}$ of leading jet, top right) $p_{T}$ of the second jet, bottom left) $p_{T}$ of the third jet (if applicable), bottom right) $\eta$ of all the jets. The simulation is normalized to the integrated luminosity of the data sample using the expected cross sections (absolute normalization). The $Z j j$ simulation is normalized so that the total number of simulated events equals the number of $Z+\geq 2$ jet events in data. . . . . . . . . . . . . . .

105 Distributions of the two leading jets (in $p_{T}$ ) in the $Z+\geq 2$ jet sample: top left) leading jet $\eta$, top right) leading jet $\phi$, bottom left) second jet $\eta$, bottom right) second jet $\phi$. The simulation is normalized to the integrated luminosity of the data sample using the expected cross sections (absolute normalization). The $Z j j$ simulation is normalized so that the total number of simulated events equals the number of $Z+\geq 2$ jet events in data. . . . . . . . . . . . . . . . . . . . .

106 Distributions of jets in the $Z+\geq 2$ jet sample: top left) $p_{T}$, top right) $H_{T}$, bottom left) $\Delta \mathrm{R}$ between the two leading jets, bottom right) invariant mass of the two leading jets. The simulation is normalized to the integrated luminosity of the data sample using the expected cross sections (absolute normalization). The $Z j j$ simulation is normalized so that the total number of simulated events equals the number of $Z+\geq 2$ jet events in data. . . . . . . . . . . . . . . 


\section{LIST OF FIGURES (Continued)}

\section{FIGURE}

PAGE

107 The resolutions of the Higgs mass given two b-tag jets. The distributions on the left have a Gaussian fit for each ZH sample. The invariant mass distributions on the right compare double b-tag jet events overlaid with exclusive single b-tag jet events. . . . . . . . . . . . .

108 Distributions of jets in the $Z+\geq 2 j$ sample with 1 exclusive b-tag jet: top left) $p_{T}$, top right) $H_{T}$, bottom left) $\Delta \mathrm{R}$ between the two leading jets, bottom right) invariant mass of the two leading jets. The simulation is normalized to the integrated luminosity of the data sample using the expected cross sections (absolute normalization). The $Z j j$ simulation is normalized so that the total number of simulated events equals the number of $Z+\geq 2$ jet events in data. . . . . . . . . . . . . .

109 Distributions of jets in the $Z+\geq 2 j$ sample with at least 2 b-tag jets: top left) $p_{T}$, top right) $H_{T}$, bottom left) $\Delta \mathrm{R}$ between the two leading jets, bottom right) invariant mass of the two leading jets. The simulation is normalized to the integrated luminosity of the data sample using the expected cross sections (absolute normalization). The $Z j j$ simulation is normalized so that the total number of simulated events equals the number of $Z+\geq 2$ jet events in data. . . . . . . . . . . . .

110 Distributions of jets in the $Z+\geq 2 j$ sample with exclusive 1 b-tag jet in the log scale: top left) $p_{T}$, top right) $H_{T}$, bottom left) $\Delta \mathrm{R}$ between the two leading jets, bottom right) invariant mass of the two leading jets. The simulation is normalized to the integrated luminosity of the data sample using the expected cross sections (absolute normalization). The $Z j j$ simulation is normalized so that the total number of simulated events equals the number of $Z+\geq 2$ jet events in data. . . . . . . .

111 Distributions of jets in the $Z+\geq 2 j$ sample with at least 2 b-tag jets: top left) $p_{T}$, top right) $H_{T}$, bottom left) $\Delta \mathrm{R}$ between the two leading jets, bottom right) invariant mass of the two leading jets. The simulation is normalized to the integrated luminosity of the data sample using the expected cross sections (absolute normalization). The $Z j j$ simulation is normalized so that the total number of simulated events equals the number of $Z+\geq 2$ jet events in data. . . . . . . . . . . .

$11295 \%$ CL cross-section upper limit for exclusive single b-tag and double b-tag samples. The observed and expected confidence levels are compared. 


\section{LIST OF FIGURES (Continued)}

\section{FIGURE}

PAGE

113 The observed 95\% CL cross-section upper limit for exclusive single b-tag and double b-tag samples. These cross-section limits are compared to standard model expectations and a CDF Run I combined lepton result.

114 Upper cross section exclusion limits divided by the SM Higgs cross section for a variety of Higgs masses. . . . . . . . . . . . . . . . . .

115 The flow of data from the inputs of the Level-2 calorimeter worker to the Level-3 system. . . . . . . . . . . . . . . . . . . . . . . . . .

$116 \quad$ FIC (Fiber Input Converter) $\ldots \ldots \ldots \ldots \ldots$

117 Level-2 alpha board. . . . . . . . . . . . . . . . .

118 Level-2 beta with $6 \mathrm{U}$ SBC and $9 \mathrm{U}$ adapter card. . . . . . . . . . . .

119 First of the five double b-tagged events.

Di-electron Inv. Mass $=100.7$; Double b-jet Inv. Mass $=59.3$;

Electron1: $p_{T}=74.2, \eta_{\text {detector }}=0.20, \phi_{\text {detector }}=4.82$, no track match;

Electron2: $p_{T}=30.4, \eta_{\text {detector }}=1.94, \phi_{\text {detector }}=4.77$, track $p_{T}=33.01$;

Jet1: $p_{T}=54.9, \eta_{\text {detector }}=0.17, \phi_{\text {detector }}=1.99$, JLIP prob. $=0.001$;

Jet2: $p_{T}=49.8, \eta_{\text {detector }}=-1.92, \phi_{\text {detector }}=2.14$, not taggable;

Jet3: $p_{T}=33.6, \eta_{\text {detector }}=-0.91, \phi_{\text {detector }}=1.16$, JLIP prob. $=0.006$; $E_{T}^{\text {miss }}=18.4, E_{T}^{\text {miss }} \phi=1.67, P V_{Z}=17.1 \ldots \ldots \ldots$

120 Second of the five double b-tagged events.

Di-electron Inv. Mass=87.0; Double b-jet Inv. Mass=165.4;

Electron1: $p_{T}=29.3, \eta_{\text {detector }}=-0.08, \phi_{\text {detector }}=4.40$, track $p_{T}=$ 20.4 ;

Electron2: $p_{T}=22.8, \eta_{\text {detector }}=2.37, \phi_{\text {detector }}=2.37$, track $p_{T}=4.88$;

Jet1: $p_{T}=120.0, \eta_{\text {detector }}=-1.09, \phi_{\text {detector }}=5.99$, not taggable;

Jet2: $p_{T}=86.9, \eta_{\text {detector }}=0.50, \phi_{\text {detector }}=3.23$, JLIP prob. $=0.001$;

Jet3: $p_{T}=41.1, \eta_{\text {detector }}=-1.18, \phi_{\text {detector }}=0.91, \mathrm{JLIP}$ prob. $=0.001$;

$E_{T}^{\text {miss }}=21.7, E_{T}^{\text {miss }} \phi=2.15, P V_{Z}=-25.6 \ldots \ldots$ 


\section{LIST OF FIGURES (Continued)}

\section{FIGURE}

PAGE

121 Third of the five double b-tagged events.

Di-electron Inv. Mass=88.7; Double b-jet Inv. Mass $=83.8$

Electron1: $p_{T}=53.2, \eta_{\text {detector }}=0.10, \phi_{\text {detector }}=5.72$, track $p_{T}=35.5$;

Electron2: $p_{T}=31.6, \eta_{\text {detector }}=-0.83, \phi_{\text {detector }}=2.31$, track $p_{T}=$ 23.9

Jet1: $p_{T}=43.3, \eta_{\text {detector }}=-0.72, \phi_{\text {detector }}=1.21$, JLIP prob. $=0.002$;

Jet2: $p_{T}=28.9, \eta_{\text {detector }}=0.48, \phi_{\text {detector }}=4.25$, JLIP prob. $=0.026$;

$E_{T}^{\text {miss }}=10.4, E_{T}^{\text {miss }} \phi=3.01, P V_{Z}=29.9 \ldots \ldots \ldots$

122 Four of the five double b-tagged events.

Di-electron Inv. Mass=85.3; Double b-jet Inv. Mass=223;

Electron1: $p_{T}=52.5, \eta_{\text {detector }}=1.55, \phi_{\text {detector }}=5.81$, tracks $p_{T}=34.4$;

Electron2: $p_{T}=34.8, \eta_{\text {detector }}=1.00, \phi_{\text {detector }}=3.28$, track $p_{T}=26.3$;

Jet1: $p_{T}=63.0, \eta_{\text {detector }}=0.69, \phi_{\text {detector }}=1.29$, JLIP prob. $=0.006$;

Jet2: $p_{T}=44.8, \eta_{\text {detector }}=-2.01, \phi_{\text {detector }}=4.17$, JLIP prob. $=0.036$;

$E_{T}^{\text {miss }}=14.7, E_{T}^{\text {miss }} \phi=2.07, P V_{Z}=-3.2 \ldots \ldots \ldots$

123 Fifth of the five double b-tagged events.

Di-electron Inv. Mass=89.6; Double b-jet Inv. Mass=65.4;

Electron1: $p_{T}=62.7, \eta_{\text {detector }}=2.02, \phi_{\text {detector }}=3.49$, track $p_{T}=433$;

Electron2: $p_{T}=20.0, \eta_{\text {detector }}=0.55, \phi_{\text {detector }}=1.13$, track $p_{T}=19.2$;

Jet1: $p_{T}=26.2, \eta_{\text {detector }}=-0.52, \phi_{\text {detector }}=0.61$, JLIP prob. $=0.020$;

Jet2: $p_{T}=20.7, \eta_{\text {detector }}=1.51, \phi_{\text {detector }}=6.03$, JLIP prob. $=0.026$;

$E_{T}^{m i s s}=18.6, E_{T}^{m i s s} \phi=0.96, P V_{Z}=12.4 \ldots \ldots \ldots$ 


\section{LIST OF ABBREVIATIONS}

$\begin{array}{ll}\text { AA } & \text { Alternative Algorithm } \\ \text { BR } & \text { Branching Ratio } \\ \text { CC } & \text { Central Calorimeter } \\ \text { CellNN } & \text { Cell Nearest Neighbor } \\ \text { CDF } & \text { Collider Detector at Fermilab } \\ \text { CERN } & \text { European Organization for Nuclear Research } \\ \text { CFT } & \text { Central Fiber Tracker } \\ \text { CH(F) } & \text { Coarse Hadronic (Fraction) } \\ \text { CIA } & \text { Control, Input/Output, Address } \\ \text { CKKW } & \text { Contrani, Krauss, Kuhn, Webber } \\ \text { COOR } & \text { Central Coordination Process } \\ & \\ \text { CPLD } & \text { Contex Programmable Logic Device } \\ & \end{array}$




\title{
LIST OF ABBREVIATIONS (Continued)
}

\author{
DOGSTAR \\ D0 GEANT Simulation of the Total Apparatus \\ Response \\ DAQ \\ Data Acquisition \\ DEC \\ Digital Equipment Corporation \\ DGLAP \\ Dokshitzer-Gribov-Lipatov-Altarelli-Parisi \\ DIS \\ Deep Inelastic Scattering \\ DMA \\ Direct Memory Access \\ DPM \\ Dual Port Memory \\ DRAM \\ Dynamic Random Access Memory \\ DSW \\ Data SWitch \\ DY \\ Drell-Yan \\ EC \\ End Calorimeter \\ EM \\ Electro Magnetic \\ EMF \\ Electro Magnetic Fraction \\ FAMUS \\ Forward Angle Muon System \\ FIC \\ Fiber Input Converter \\ FH \\ Fine Hadronic \\ FIFO \\ First In, First Out \\ Fermilab \\ Fermi National Accelerator Laboratory \\ xxvi
}




\section{LIST OF ABBREVIATIONS (Continued)}

\begin{tabular}{|c|c|}
\hline FNAL & Fermi National Accelerator Laboratory \\
\hline FPGA & Field Programmable Gate Array \\
\hline FPS & Forward PreShower \\
\hline FSR & Final State Radiation \\
\hline GEANT & GEometry ANd Tracking \\
\hline GWS & Glashow, Weinberg, Salam \\
\hline $\mathrm{HAD}$ & Hadronic \\
\hline HotF & Hot Fraction \\
\hline HTF & Histogramming Track Finder \\
\hline ICD & Inter Cryostat Detector \\
\hline ICR & Inter Cryostat Region \\
\hline ISR & Initial State Radiation \\
\hline IDE & Integrated Drive Electronics \\
\hline $\mathrm{I} / \mathrm{O}$ & Input/Output \\
\hline ISA & Industry Standard Architecture \\
\hline JES & Jet Energy Scale \\
\hline L1 & Level-1 \\
\hline L1Cal & Level-1 Calorimeter \\
\hline
\end{tabular}

xxvii 


\section{LIST OF ABBREVIATIONS (Continued)}

\begin{tabular}{|c|c|}
\hline L1CTT & Level-1 Central Track Trigger \\
\hline L1Muon & Level-1 Muon \\
\hline L2 & Level-2 \\
\hline L2Cal & Level-2 Calorimeter \\
\hline L2CTT & Level-2 Central Track Trigger \\
\hline L2GBL & Level-2 Global \\
\hline L2MUC & Level-2 Muon Central \\
\hline L2MUF & Level-2 Muon Forward \\
\hline L2Muon & Level-2 Muon \\
\hline L2PS & Level-2 PreShower \\
\hline L2STT & Level-2 Silicon Track Trigger \\
\hline L3 & Level-3 \\
\hline L3DAQ & Level-3 Data Acquisition \\
\hline $\mathrm{LBN}$ & Luminosity Block Number \\
\hline Linac & Linear Accelerator \\
\hline $\mathrm{LL}$ & Leading Logarithmic \\
\hline LLA & Leading Log Approximation \\
\hline MB & Minimum Bias \\
\hline
\end{tabular}

xxviii 


\section{LIST OF ABBREVIATIONS (Continued)}

\begin{tabular}{|c|c|}
\hline MBT & Magic Bus Transceiver \\
\hline MBus & Magic Bus \\
\hline $\mathrm{MC}$ & Monte Carlo \\
\hline MCFM & Monte Carlo for FeMtobarn processes \\
\hline MDT & Mini Drift Tube \\
\hline MG & Massless Gap \\
\hline NLO & Next-to-Leading Order \\
\hline PCI & Peripheral Component Interconnect \\
\hline $\mathrm{PDF}$ & Parton Distribution Function \\
\hline PDT & Proportional Drift Tube \\
\hline $\mathrm{PIO}$ & Programmed Input Output \\
\hline PLD & Programmable Logic Device \\
\hline PV & Primary Vertex \\
\hline $\mathrm{RF}$ & Radio Frequency \\
\hline RISC & Reduced Instruction Set Computing \\
\hline $\mathrm{ROM}$ & Read Only Memory \\
\hline QCD & Quantum Chromo Dynamics \\
\hline QED & Quantum Electro Dynamics \\
\hline
\end{tabular}

xxix 


\section{LIST OF ABBREVIATIONS (Continued)}

\begin{tabular}{|c|c|}
\hline QFT & Quantum Field Theory \\
\hline SBC & Single Board Computer \\
\hline SCL(init) & Serial Command Link (Initialize) \\
\hline Scone & Simple Cone \\
\hline SIMM & Single In-line Memory Module \\
\hline SLIC & Second Level Input Computer \\
\hline SM & Standard Model \\
\hline SMT & Silicon Microstrip Tracker \\
\hline SROM & Serial Read Only Memory \\
\hline TCC & Trigger Control Computer \\
\hline TDR & Technical Design Report \\
\hline TFW & Trigger FrameWork \\
\hline $\mathrm{TT}$ & Trigger Tower \\
\hline VBD & VME Buffer Driver \\
\hline VITA & VMEbus International Trade Association \\
\hline VLPC & Visible Light Photon Counter \\
\hline VME & VERSA Module Eurocard \\
\hline WAMUS & Wide Angle Muon System \\
\hline
\end{tabular}




\section{SUMMARY}

This analysis describes a search for a standard model Higgs boson produced in association with a $Z$ boson through the decay mode $Z H \rightarrow e^{+} e^{-} b \bar{b}$ in $p \bar{p}$ collisions at $\sqrt{s}=1.96 \mathrm{TeV}$ at the Fermilab Tevatron Collider. The data sample used in this analysis corresponds to 452 $\mathrm{pb}^{-1}$ of integrated luminosity accumulated with the $\mathrm{D} \varnothing$ detector. Agreement between data and standard model predictions is observed. A 95\% confidence level upper exclusion limit for the $\sigma(p \bar{p} \rightarrow Z H) \times \mathrm{BR}(H \rightarrow b \bar{b})$ channel is set between 3.2-8.2 pb for Higgs masses of 105 to $145 \mathrm{GeV}$. 


\section{CHAPTER 1}

\section{INTRODUCTION}

The second most sensitive production channel $^{1}$ at the Tevatron for a Higgs mass below $140 \mathrm{GeV}$ is the associated production of a Higgs boson with a $Z$ boson, which has a hadronic branching ratio of $70 \%$, but the four jet event (assuming $H \rightarrow b \bar{b}$ ) is overwhelmed by quantum chromodynamics (QCD) multi-jet production. This analysis has the benefit of using electrons to identify the $\mathrm{Z}$ boson which provide a clear signature with minimum background contributions. The electrons from the Z-boson decays have high transverse momenta allowing the events to be selected online with nearly $100 \%$ efficiency.

The first results for a Higgs search in the $Z H \rightarrow e^{+} e^{-} b \bar{b}$ channel using a data sample of 452 $\mathrm{pb}^{-1}$ collected with the $\mathrm{D} \varnothing$ detector are presented. Two electrons from $Z$ boson decays and two jets with at least one b-tag are required. The dominant backgrounds to $Z H$ production are due to associated production of a $Z$ boson with two jets, and top-quark production, and di-boson production. No excess of events is observed in data. Therefore, cross section exclusion limits are derived.

This thesis is organized as follows:

- A theoretical introduction to the analysis is given in Chapter 2.

\footnotetext{
${ }^{1}$ The associated production of the Higgs boson with a $W$ boson is the most sensitive production channel for electron decays.
} 
- The Experimental Apparatus describing the Tevatron collider and DØ detector is presented in Chapter 3.

- The DØ Trigger and Data Acquisition Systems describes the collection of data online during high energy collisions in Chapter 4.

- The Offline Event Reconstruction and Object Identification is explained in Chapter 5.

- The Monte Carlo Event Simulations that are used for this analysis are discussed in Chapter 6 .

- Data and MC Samples are explained and general comparison plots are shown in Chapter 7.

- Object Reconstruction and Selection Efficiencies are given in Chapter 8.

- Details of the High Level Analysis, the search for the Higgs boson, are described Chapter 9.

- Uncertainties in the search and an upper ZH Cross-Section Limit and are described in Chapter 10 .

- A Summary and Conclusion is given in Chapter 11.

Throughout this dissertation (unless stated otherwise), units of $\hbar=\mathrm{c}=1$ are used. 


\section{CHAPTER 2}

\section{THEORY}

The Standard Model $(\mathrm{SM})(1 ; 2)$ is a theoretical description of natural phenomena of the subatomic world. The SM is a quantum field theory (QFT) where all particles are described by quantum fields. QFT combines quantum mechanics with special relativity. In QFT, interactions manifest themselves by imposing symmetry conditions on the quantum fields. The SM is built on the $\mathrm{SU}(3)^{1} \times \mathrm{SU}(2) \times \mathrm{U}(1)$ symmetry of three gauge groups. The $\mathrm{SU}(3)$ group describes quantum chromodynamics, the strong interaction. The SU(2) group describes quantum flavor dynamics, the weak interaction. The U(1) group describes quantum electrodynamics, the electromagnetic (EM) interaction.

The SM has undergone great scrutiny in the last twenty years. It has held up well as a model, but the last remaining piece, called the Higgs boson, has not been experimentally detected. The SM predicts a Higgs field which permeates the vacuum and interacts with particles giving them their mass after electroweak symmetry breaking. Although, the SM predicts the manifestation of a Higgs particle, it does not predict its mass.

\footnotetext{
${ }^{1} \mathrm{~S}$ - simple - the determinant of the matrix is equal to 1 , $\mathrm{U}$ - unitary $-U^{-1}=U^{\dagger}, 3-3 \times 3$ matrix
} 


\subsection{The Standard Model}

\subsubsection{Quarks and Leptons}

The SM has two groups of particles, leptons and quarks $(3 ; 4)$, as fundamental constituents. Each group contains six spin- $\frac{1}{2}$ particles grouped into three distinct doublets, or families:

$$
\begin{gathered}
\text { Leptons : }\left(\begin{array}{c}
\nu_{e} \\
e
\end{array}\right),\left(\begin{array}{c}
\nu_{\mu} \\
\mu
\end{array}\right),\left(\begin{array}{c}
\nu_{\tau} \\
\tau
\end{array}\right) \\
\text { Quarks : }\left(\begin{array}{c}
\mathrm{u} \\
\mathrm{d}
\end{array}\right),\left(\begin{array}{l}
\mathrm{c} \\
\mathrm{s}
\end{array}\right),\left(\begin{array}{c}
\mathrm{t} \\
\mathrm{b}
\end{array}\right)
\end{gathered}
$$

The lepton group consists of the $e$ (electron), $\mu$ (muon), $\tau$ (tau) leptons with an electric charge of $-\mathrm{e}^{1}$, and their associated neutrinos, with zero electric charge. The quark group consists of six flavors, u (up), d (down), c (charm), s (strange), t (top), and b (bottom). They carry fractional electric charges of $+\frac{2}{3} \mathrm{e}\left(\mathrm{u}, \mathrm{c}\right.$, and t quarks) or $-\frac{1}{3} \mathrm{e}(\mathrm{d}, \mathrm{s}$, and b quarks). Table I shows some of the properties of the three families of quarks and leptons. For each particle, there exists an associated anti-particle which has the same mass but opposite charge.

The quarks form bound states called hadrons by combining three quarks into a half-integral spin baryon or by combining a quark-antiquark pair into an integral spin meson. Protons (made up of two u-quarks and one d-quark) and neutrons (made up of two d-quarks and one u-quark) are the most common example of baryons. Neutral pions (made up of one u-quark

$$
{ }^{1} \mathrm{e}=1.602 \times 10^{-19} \text { Coulombs }
$$


and one anti-u quark) and charged pions (made up of one u-quark and one anti-down quark (for a positive one electric charge)) are the most common example of mesons.

\begin{tabular}{|cc|cc|cc|c|c|}
\hline Family 1 & & Family 2 & & Family 3 & Electric & \\
\hline Quark & Mass $(\mathrm{GeV})$ & Quark & Mass $(\mathrm{GeV})$ & Quark & Mass $(\mathrm{GeV})$ & Charge & Forces \\
\hline up & $3 \times 10^{-3}$ & charm & 1.3 & top & 175 & $+\frac{2}{3}$ & PWG \\
down & $6 \times 10^{-3}$ & strange & 0.1 & bottom & 4.3 & $-\frac{1}{3}$ & PWG \\
\hline electron & $5.1 \times 10^{-4}$ & muon & 0.106 & tau & 1.8 & -1 & PW \\
$\nu_{e}$ & $\leq 10^{-8}$ & $\nu_{\mu}$ & $\leq 2 \times 10^{-4}$ & $\nu_{\tau}$ & $\leq 0.02$ & 0 & W \\
\hline
\end{tabular}

TABLE I

FUNDAMENTAL FERMION BUILDING BLOCKS OF MATTER. THE FORCES ARE MEDIATED BY THE PHOTON (P), WEAK BOSONS (W), AND GLUONS (G).

\subsubsection{Interactions}

There are four fundamental interactions in nature: strong, electromagnetic, weak, and gravitational which are mediated by bosons. The photon is the carrier of the electromagnetic interaction which, holds electrons around the nucleus of an atom. The eight gluons are the carriers of the strong interaction which hold the protons and neutrons together in a nucleus. The $\mathrm{W}^{ \pm}$and $\mathrm{Z}$ bosons carry the weak interaction which is responsible for the $\beta$ decay. The graviton boson mediates the gravitational interaction. The SM does not include gravity in its mathematical formalism. Table II summarizes the three interactions described by the SM. 
Normalizing the strength of strong interaction to one, the electromagnetic interaction is a hundred times weaker and the weak interaction is a million times weaker.

The fundamental interactions in nature manifest themselves by imposing symmetry conditions on the quantum fields representing the respective interactions. The SM Lagrangian is required to be invariant under certain types of symmetry transformations, such as local gauge transformations. The application of an arbitrary gauge to the fields is called a gauge transformation. A local gauge transformation depends on space and time in an completely arbitrary way. In order to ensure local gauge invariance in the Lagrangian, gauge fields are introduced. These gauge fields lead to spin-1 bosons, the carriers of the three fundamental interactions.

For the $\mathrm{U}(1)$ group, demanding that there is no observable change arising from changing the complex phase of the field at each point in space-time independently (local gauge transformation), creates what we observe as electromagnetism. Local gauge invariance under an $\mathrm{SU}(2)$ group transformation introduces three spin-1 gauge bosons $W^{1}, W^{2}$ and $W^{o}$.

\begin{tabular}{|c|c|c|c|c|c|c|c|}
\hline Force & Boson & Group & Mass & $\begin{array}{c}\text { EM } \\
\text { Charge }\end{array}$ & $\mathrm{V}(\mathrm{r})$ & $\begin{array}{c}\text { Effective } \\
\text { Range }(\mathrm{m})\end{array}$ & $\begin{array}{c}\text { Relative } \\
\text { Strength }\end{array}$ \\
\hline strong & gluon & $\mathrm{SU}(3)$ & 0 & 0 & $\mathrm{r}$ & $10^{-15}$ & 1 \\
weak & $\mathrm{W}^{ \pm}, \mathrm{Z}$ & $\mathrm{SU}(2)$ & $80.4,91.2$ & $\pm 1,0$ & $\frac{e^{-M r}}{r}$ & $10^{-12}$ & $10^{-6}$ \\
EM & photon & $\mathrm{U}(1)$ & 0 & 0 & $1 / r$ & $\infty$ & $10^{-2}$ \\
\hline
\end{tabular}

TABLE II

FUNDAMENTAL INTERACTIONS OF NATURE IN THE SM. 


\subsubsection{Strong Interaction}

Quantum Chromo Dynamics (QCD) $(5 ; 6)$ is the QFT describing strong interactions. It is based on a $\mathrm{SU}(3)(7 ; 8)$ gauge field, which leads to eight mediating massless gauge bosons called gluons. Quarks carry a different type of "charge" called color. Each (anti)quark can carry (anti)red, (anti)green, or (anti)blue color charge. Gluons carry a combination of color and anticolor charge. As carriers of the color charge, gluons can couple to each other. This derives from the non-Abelian ${ }^{1}$ character of the gauge theory. Quarks and gluons are collectively referred to as partons.

An important property of QCD is that the strength of the strong coupling constant increases with decreasing energy scale. At low energies and long distances the interaction becomes too strong to be treated within the framework of perturbation theory. This leads to confinement, which assumes that all objects which carry color can never be found as free particles in nature and that they are confined into color-neutral composite hadrons.

Experimentally, quarks and gluons are observed as jets of color-neutral hadrons. When a parton emerges from a hard scattering, gluons are radiated along its path, and subsequently produce quark-antiquark pairs, forming a parton shower. Ultimately, the partons combine into a jet of hadrons moving in the direction close to that of the original parton. The final step is called hadronization.

\footnotetext{
${ }^{1}$ The term non-Abelien refers to the non-commutative generators of the SU(3) symmetry group.
} 
Another important property of QCD is asymptotic freedom $(9 ; 10)$. The property of asymptotic freedom describes the weakening of the effective quark-gluon coupling at short-distances (or large momentum transfers). This feature of asymptotic freedom allows the perturbative treatment of strong interactions in obtaining predictions for processes that are dominated by short-distance interactions (11).

The strong coupling constant, $\alpha_{s}$ can be expressed to leading-log ${ }^{1}$ in $Q^{2}$ by:

$$
\alpha_{s}\left(Q^{2}\right)=\frac{12 \pi}{\left(11 c-2 n_{f}\right) \log \left(\frac{Q^{2}}{\lambda_{Q C D}^{2}}\right)}
$$

$\mathrm{Q}$ is the magnitude of the momentum transfered in the interaction. $\mathrm{n}_{f}$ is the number of flavors of quarks (6 in the $\mathrm{SM})$, $\mathrm{c}$ is the number colors (3 in the $\mathrm{SM}) . \lambda_{Q C D}$ is a $\mathrm{QCD}$ scale parameter and is parameterized in Equation 2.2.

$$
\lambda_{Q C D}^{2}=\mu_{r}^{2} \exp \frac{-12 \pi}{\left(11 c-2 n_{f}\right) \alpha_{s}\left(\mu_{r}^{2}\right)}
$$

$\mu_{r}^{2}$ is an arbitrary renormalization scale used to regulate divergences in the perturbative calculation of $\alpha_{s}$. Equation 2.1 shows that the strength of the strong coupling decreases with increasing momentum transfer $Q^{2}$. Therefore, quarks and gluons are asymptotically free when probed at high energies. Theoretical work on asymptotic freedom by Gross, Politzer, and

\footnotetext{
${ }^{1}$ The term "leading-log" is used to indicate an all-orders calculation in which only the leading logarithm terms are retained.
} 
Wilczek was rewarded with the 2004 Nobel Prize. On the other hand, as $Q^{2}$ approaches $\lambda_{Q C D}$, the coupling becomes large and perturbative calculations are no longer possible.

\subsubsection{Electroweak Interaction}

The weak gauge bosons are heavy particles (12), therefore, fermions only experience the weak interaction at very short ranges.

$$
\begin{gathered}
m_{W^{ \pm}}=80.403 \pm 0.029 \mathrm{GeV} \\
m_{Z^{\circ}}=91.1876 \pm 0.0021 \mathrm{GeV}
\end{gathered}
$$

Neutrinos can only interact weakly since they do not carry charge or color. The $Z$ bosons allow for neutral current interactions between two neutrinos. $W^{ \pm}$bosons only allow for weak flavor changing charged currents. Therefore, quarks can change flavor $\mathrm{t} \rightarrow \mathrm{b}, \mathrm{c} \rightarrow \mathrm{s}, \mathrm{u} \rightarrow \mathrm{d}$, but not $\mathrm{t} \nrightarrow \mathrm{c}$ or $\mathrm{c} \nrightarrow \mathrm{u}$. The photon couples to all particles that carry electric charge.

A QFT combining the electromagnetic with the weak interaction was first developed by Glashow, Weinberg, and Salam (GWS theory, Noble Prize in 1979). Later, 't Hooft and Veltman were able to prove that the theory is renormalizable (Noble Prize in 1999). The $\mathrm{SU}(2)_{L} \times \mathrm{U}(1)_{Y}{ }^{1}$ group combines a $\mathrm{U}(1)$ group with an $\mathrm{SU}(2)$ group. Local gauge invariance under $\mathrm{SU}(2)_{L}$ transformations introduces three massless spin- 1 gauge bosons $\mathrm{W}^{1}, \mathrm{~W}^{2}$, and $\mathrm{W}^{o}$. Adding the

\footnotetext{
${ }^{1} \mathrm{~L}$ for left handed chirality and $\mathrm{Y}$ for hypercharge $Q=T^{3}+\frac{Y}{2}$, T for isospin.
} 
$\mathrm{U}(1)_{Y}$ group introduces another gauge boson $\mathrm{B}^{o}$. Experimental observables $\mathrm{W}^{ \pm}, \mathrm{Z}^{o}$, and $\gamma$ are linear combinations of the $\mathrm{W}^{1}, \mathrm{~W}^{2}, \mathrm{~W}^{o}$, and $\mathrm{B}^{o}$ fields:

$$
\begin{array}{r}
\gamma=W^{o} \sin \theta_{W}+B^{o} \cos \theta_{W} \\
Z^{o}=W^{o} \cos \theta_{W}-B^{o} \sin \theta_{W} \\
W^{ \pm}=\sqrt{\frac{1}{2}}\left(W^{1} \pm i W^{2}\right)
\end{array}
$$

Where $\theta_{W}$ is the weak mixing angle or Weinberg angle. The underlying group of the weak theory is non-Abelian.

Up to this point the electroweak theory is incomplete since all the particles of the theory are massless. Mass terms cannot be introduced into the Lagrangian describing the system, since this would destroy the local gauge invariance of the Lagrangian. This problem is resolved by the Higgs mechanism, which introduces spontaneous symmetry breaking, thereby giving mass to the gauge bosons $\left(W^{ \pm}\right.$and $\left.\mathrm{Z}\right)$, the quarks, and the leptons.

\subsubsection{The Higgs Mechanism}

The SM suggests that mass can not be viewed as an intrinsic property of a particle, but rather that mass comes from some interaction. The SM Lagrangian gives mass to the fermions and the weak vector bosons by introducing the Higgs field which interacts with the fermions and weak vector bosons. The mass terms manifest themselves in the SM by applying spontaneous symmetry breaking with local gauge invariance to the Higgs field and the electroweak fields. This process is called the Higgs mechanism $(13 ; 14 ; 15)$. 
The SM Higgs field is a scalar complex doublet field with four degrees of freedom.

$$
\phi=\left(\begin{array}{c}
\phi^{+} \\
\phi^{o}
\end{array}\right)
$$

The Higgs couples to the massless gauge fields:

$$
\mathcal{D}_{\mu}=\partial_{\mu}+i \frac{g^{\prime}}{2} \mathcal{B}_{\mu} Y+i \frac{g}{2} \vec{\tau} \cdot \overrightarrow{W_{\mu}}
$$

The Higgs field includes a local gauge invariant potential added to the Lagrangian:

$$
\mathcal{L}_{\text {scalar }}=\left(\mathcal{D}^{\mu} \phi\right)^{\dagger}\left(\mathcal{D}_{\mu} \phi\right)-\mu^{2}\left(\phi^{\dagger} \phi\right)-\lambda\left(\phi^{\dagger} \phi\right)^{2}
$$

When $\lambda>0$ and $\mu^{2}>0$ there is the trivial minimum at the center of the potential, $\phi^{\dagger} \phi=$ 0. If $\lambda>0$ and $\mu^{2}<0$ then the Higgs field potential has infinite minima, all satisfying the condition $\phi^{\dagger} \phi=\frac{\mu^{2}}{2 \lambda}$. Expanding around a minimum of the Higgs field potential (Equation 2.9) the Lagrangian can be written in such a way that it gives origin to the SM gauge boson mass terms.

$$
\phi=\left(\begin{array}{c}
\phi^{+} \\
\phi^{o}
\end{array}\right) \rightarrow \frac{1}{\sqrt{2}}\left(\begin{array}{c}
0 \\
\nu+\eta
\end{array}\right) \quad \nu^{2}=\frac{-\mu^{2}}{\lambda}
$$


This choice of vacuum spontaneously breaks the symmetry and the Lagrangian is no longer

locally gauge invariant. The $W^{ \pm}$and $Z$ bosons each absorb a degree of freedom and acquire mass. The mass terms of the bosons are:

$$
M_{W^{ \pm}}=\frac{g \nu}{2} \quad M_{Z}=\frac{g \nu}{2 \cos \theta_{W}} \quad M_{H}=\sqrt{-2 \mu^{2}}
$$

Fermion masses are generated through the introduction of Yukawa couplings in Higgs-fermion vertices as shown in Equation 2.11.

$$
\mathcal{L}_{Y u k a w a}=-G_{e}\left(\bar{R}\left(\phi^{\dagger} L\right)+(\bar{L} \phi) R\right)
$$

\subsubsection{Constraints on the Higgs Mass}

The search for the neutral scalar Higgs particle is the topic of this dissertation. The SM does not predict a mass for the Higgs boson, but an upper limit of about $700 \mathrm{GeV}$ can be inferred from unitarity constraints from di-boson scattering (i.e. $V_{L} V_{L} \rightarrow V_{L} V_{L}\left(V=W^{ \pm}, Z\right)$ ), since the scattering amplitudes grow as the square of the Higgs mass.

Another upper bound on the mass of the Higgs boson can be derived from the requirement that the SM is valid up to a scale $\Lambda$, before perturbation theory breaks down and new nonperturbative phenomena dominate the predictions of the theory. If the SM is required to be valid up to the Planck scale of $10^{16} \mathrm{GeV}$, the Higgs mass has to be $134<M_{H}<177 \mathrm{GeV}$. For a minimal cut-off $\Lambda$ about $1 \mathrm{TeV}$, an upper bound of about $700 \mathrm{GeV}$ can be obtained from renormalization group analysis and lattice simulations of the SM Higgs sector. 
The current best limit on the mass of a SM Higgs boson is $M_{H}>114.4 \mathrm{GeV}$ at $95 \% \mathrm{CL}$ from the LEP experiments (17). The $W^{ \pm}$mass is sensitive through higher loop corrections to the mass of the top quark and the mass of the Higgs boson. The $W^{ \pm}$mass corrections depend logarithmically with the Higgs mass and quadratically with the top-quark mass. The $W^{ \pm}$mass and the top-quark mass measurements provide constraints for the SM Higgs boson mass as shown in Figure $1(16)$. The $(\Delta \chi)^{2}=\chi^{2}-\chi_{\min }^{2}$ plot of global fits to several precision measurements of $\mathrm{EW}$ parameters, include the $\mathrm{M}_{W^{ \pm}}$and $\mathrm{M}_{t o p}$, shows that the expectation of the Higgs mass (within the SM) is $85_{-28}^{+39}$ at the $68 \% \mathrm{CL}$ and $<199 \mathrm{GeV}$ at the $95 \% \mathrm{CL}$. 

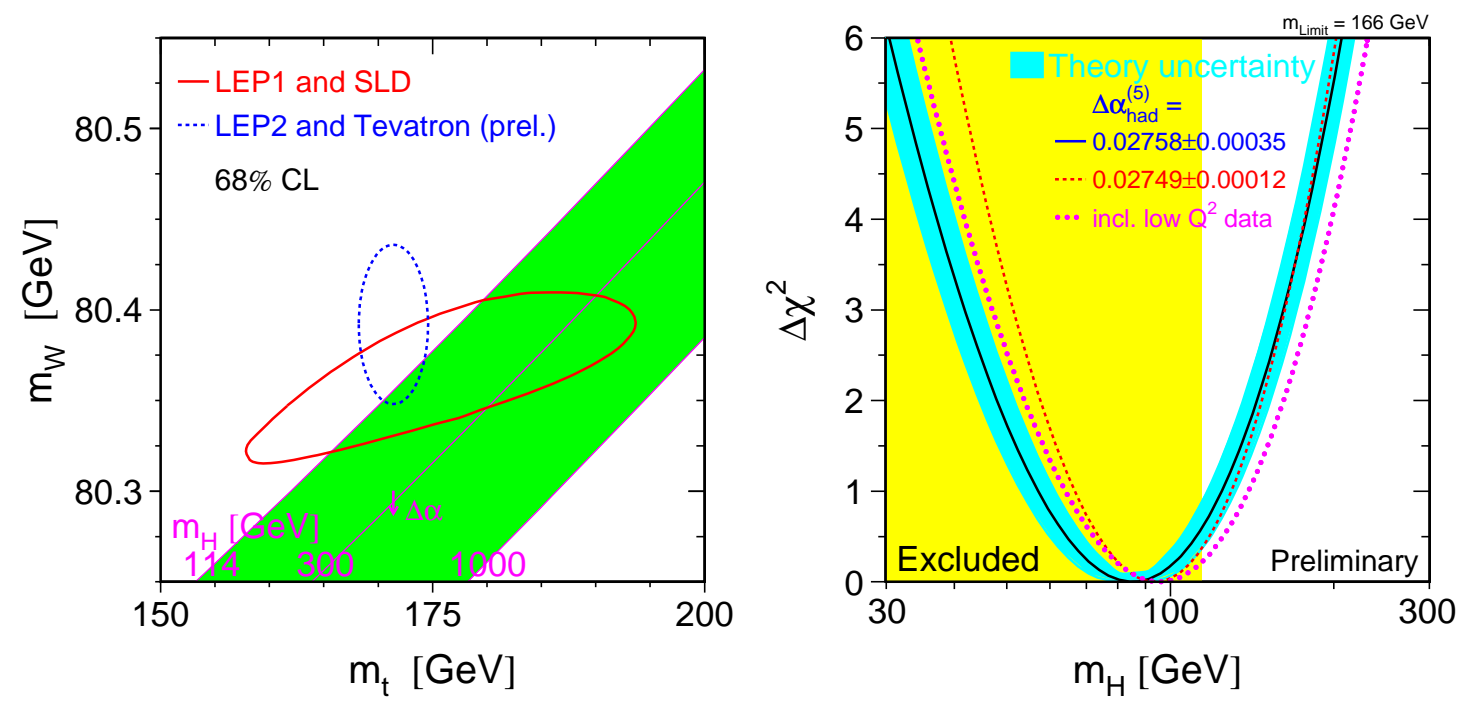

Figure 1. Limits on the Higgs boson mass from electroweak measurements. The figure on the left shows the relationship between the $\mathrm{M}_{H}$ to the mass of the $W^{ \pm}$and the top quark. The figure on the right uses high precision electroweak data to constrain the mass of the Higgs.

The $M_{H}$ is constrained to be greater than $114.4 \mathrm{GeV}$ from LEP experiments (shown in yellow). 


\subsection{Search for the Higgs at the Tevatron}
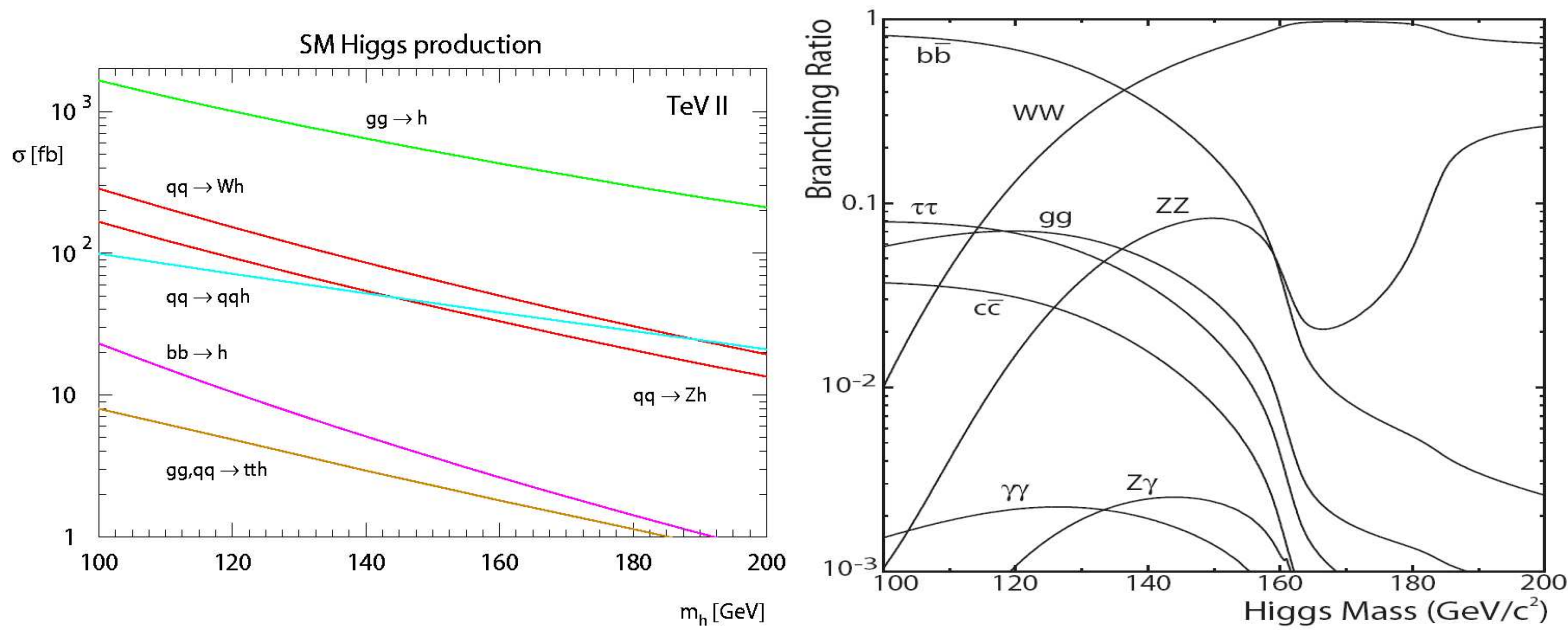

Figure 2. Production cross section of the Higgs boson (left plot) at the Tevatron and the Higgs boson branching ratio (right plot).

Shown in Figure 2, the most probable production of the Higgs boson at the Tevatron is through gluon fusion (18), followed by the associated production with a $W^{ \pm}$boson, and associated production with a $Z$ boson. For a Higgs mass below $135 \mathrm{GeV}$, the most probable decay of the Higgs boson is into $b \bar{b}$ quarks. For a Higgs mass above $135 \mathrm{GeV}$, the most probable decay of the Higgs boson is into a pair of $W^{ \pm 1}$

${ }^{1}$ one $W^{ \pm}$boson is virtual until $M_{H}>2 M_{W^{ \pm}}$bosons. 
For a Higgs mass below $135 \mathrm{GeV}$, the gluon fusion production channel is hopeless due to the large backgrounds of $b \bar{b}$ production from other sources. The most promising modes for a light Higgs discovery at the Tevatron are those where the Higgs is produced in association with a $W^{ \pm}$or $Z$ boson, with $(W / Z) \rightarrow$ leptons and $H \rightarrow b \bar{b}$.

\subsubsection{Higgs Sensitivity Studies}

The expected status of the Higgs search at the end of the Tevatron run should yield a total integrated luminosity of $8 \mathrm{fb}^{-1}$ per experiment (Figure 3). If no Higgs signal is seen, then the 95\% CL lower limit on the SM Higgs mass is projected to be $M_{H}>138 \mathrm{GeV}$. In order to have evidence of the Higgs boson at the $3 \sigma$ level, one must have $M_{H}<129 \mathrm{GeV}$. In order to discover the Higgs boson at the $5 \sigma$ level, one must have $M_{H}<117 \mathrm{GeV}$. This analysis uses a data sample of $\sim 0.5 \mathrm{fb}^{-1}$ of integrated luminosity.

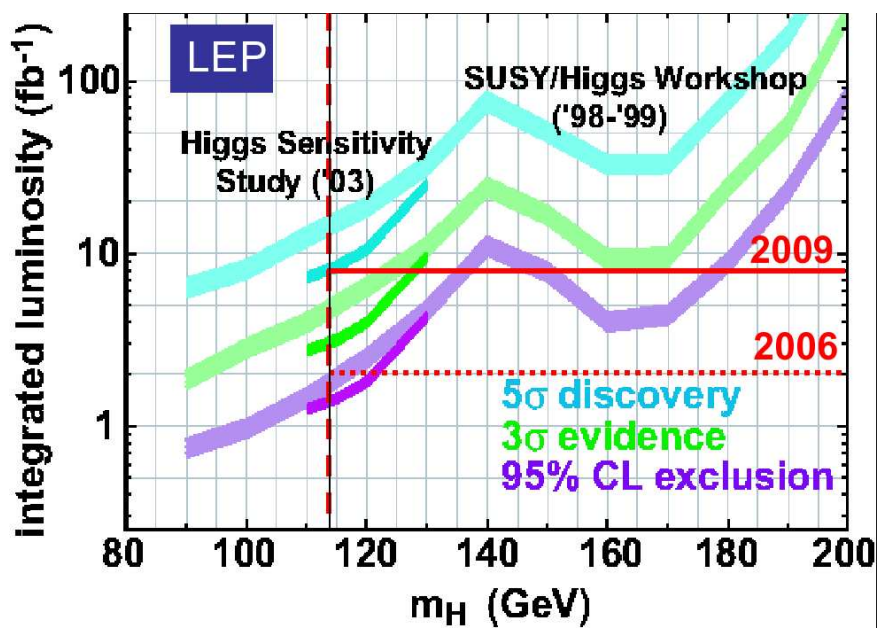

Figure 3. Projected Tevatron limits on the SM Higgs boson mass (in GeV) as a function of integrated luminosity per experiment with $95 \%$ CL exclusion, $3 \sigma$ evidence, and $5 \sigma$ discovery. 


\subsubsection{Overview of the $\mathrm{ZH}$ Search}

This analysis searches for a light mass Higgs boson decaying into $b \bar{b}$, in a mass range between 105 and $145 \mathrm{GeV}$, produced in association with a $\mathrm{Z}$ boson decaying into an electron and positron pair. This channel benefits from using electrons to identify the $Z$ bosons which provide a clear signature with minimum background contributions. The electrons from the $Z$ boson decays have high transverse momenta allowing the events to be selected online with nearly $100 \%$ efficiency.

One of the leading-order Feynman diagrams for the Higgs signal in the ZH channel is shown in Figure 4. There are many physics processes with similar signature of two electrons and two b jets which contribute to the background for this channel. The three main processes $\mathrm{Z}\left(\rightarrow e^{+} e^{-}\right) b \bar{b}, \mathrm{t}\left(\rightarrow b W^{+}\left(\rightarrow e^{+} \nu\right)\right) \bar{t}\left(\rightarrow \bar{b} W^{-}\left(\rightarrow e^{-} \bar{\nu}\right)\right)$, and $\mathrm{Z}\left(\rightarrow e^{+} e^{-}\right) \mathrm{Z}(\rightarrow b \bar{b})$ are shown in Figure 5. Another source of background to the $\mathrm{ZH}$ signal comes from $Z+$ jets processes where the light-quark jets have been misidentified as b jets in data. Such processes include $\mathrm{Z}\left(\rightarrow e^{+} e^{-}\right) \mathrm{qq}$, $\mathrm{Z}\left(\rightarrow e^{+} e^{-}\right) \mathrm{Z}(\rightarrow q \bar{q})$, and $\mathrm{Z}\left(\rightarrow e^{+} e^{-}\right) \mathrm{W}\left(\rightarrow q \overline{q^{\prime}}\right)$ where q can be any quark flavor other than the b quark. Finally, the $\mathrm{t}\left(\rightarrow b W^{+}\left(\rightarrow e^{+} \nu\right)\right) \mathrm{t}\left(\rightarrow \bar{b} W^{-}\left(\rightarrow q \overline{q^{\prime}}\right)\right)$ production could give a final state that fakes the ZH signal, hence contributing to the background. 


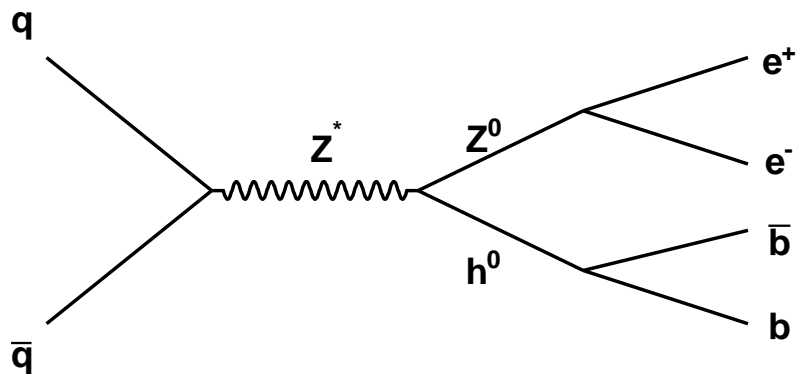

Figure 4. A Feynman diagram or the $\mathrm{Z}\left(\rightarrow e^{+} e^{-}\right) \mathrm{H}(\rightarrow b \bar{b})$ channel. 

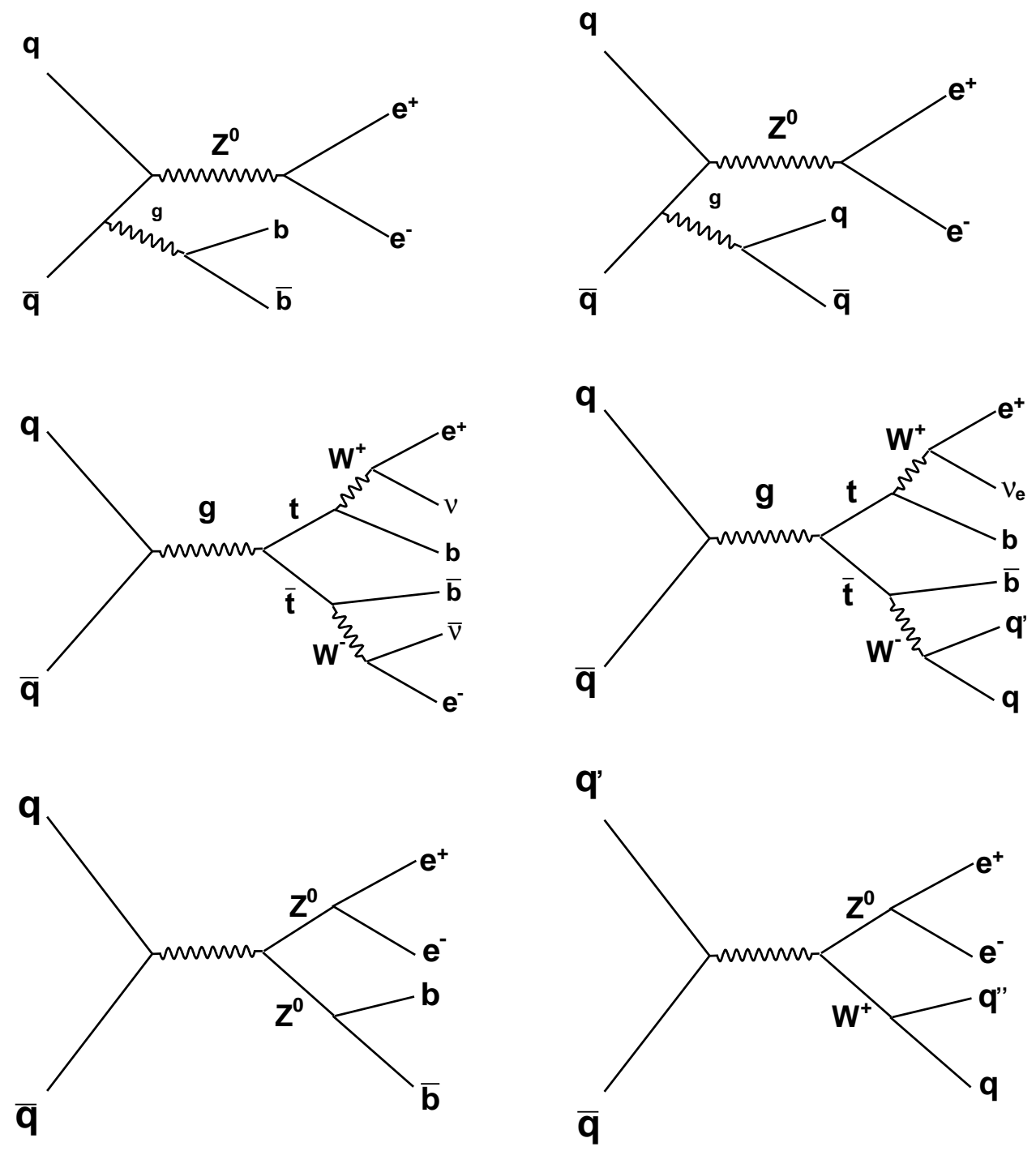

Figure 5. Feynman diagrams of physics processes that contribute to the background. 


\section{CHAPTER 3}

\section{EXPERIMENTAL APPARATUS}

The Fermi National Accelerator Laboratory (Fermilab) (19) operates the world's highest energy proton-antiproton collider, the Tevatron. In this chapter, the chain of accelerators that achieve a center-of-mass collision energy of $1.96 \mathrm{TeV}$ is described. An overview of the D $\varnothing$ detector, which is built around one of the interaction regions where proton and antiprotons collide, is given.

The data used in this analysis were recorded with the $\mathrm{D} \varnothing$ detector during the data taking period known as Run II, which officially began in March 2001.

\subsection{The Accelerators}

The Fermilab particle accelerators $(20 ; 21)$ consist of seven separate devices, as shown in Figure 6: the Cockcroft-Walton pre-accelerator; the Linac linear accelerator; the Booster synchrotron; the Main Injector; the Antiproton Source; the antiproton Recycler; and the Tevatron. The Antiproton Source has three components: an antiproton target; a Debuncher; and an Accumulator. An overview of each device is given in the following sections.

\subsubsection{The Cockcroft Walton}

The Cockcroft-Walton pre-accelerator starts by turning hydrogen gas $\left(\mathrm{H}_{2}\right)$ into hydrogen ions $\left(\mathrm{H}^{-}\right)$. The hydrogen gas is pulsed at $15 \mathrm{~Hz}$ with a pulse duration of $80 \mu s$. The $\mathrm{H}^{-}$ ions are produced by a Magnetron Surface Plasma Source (MSPS). The MSPS (Figure 7) is 


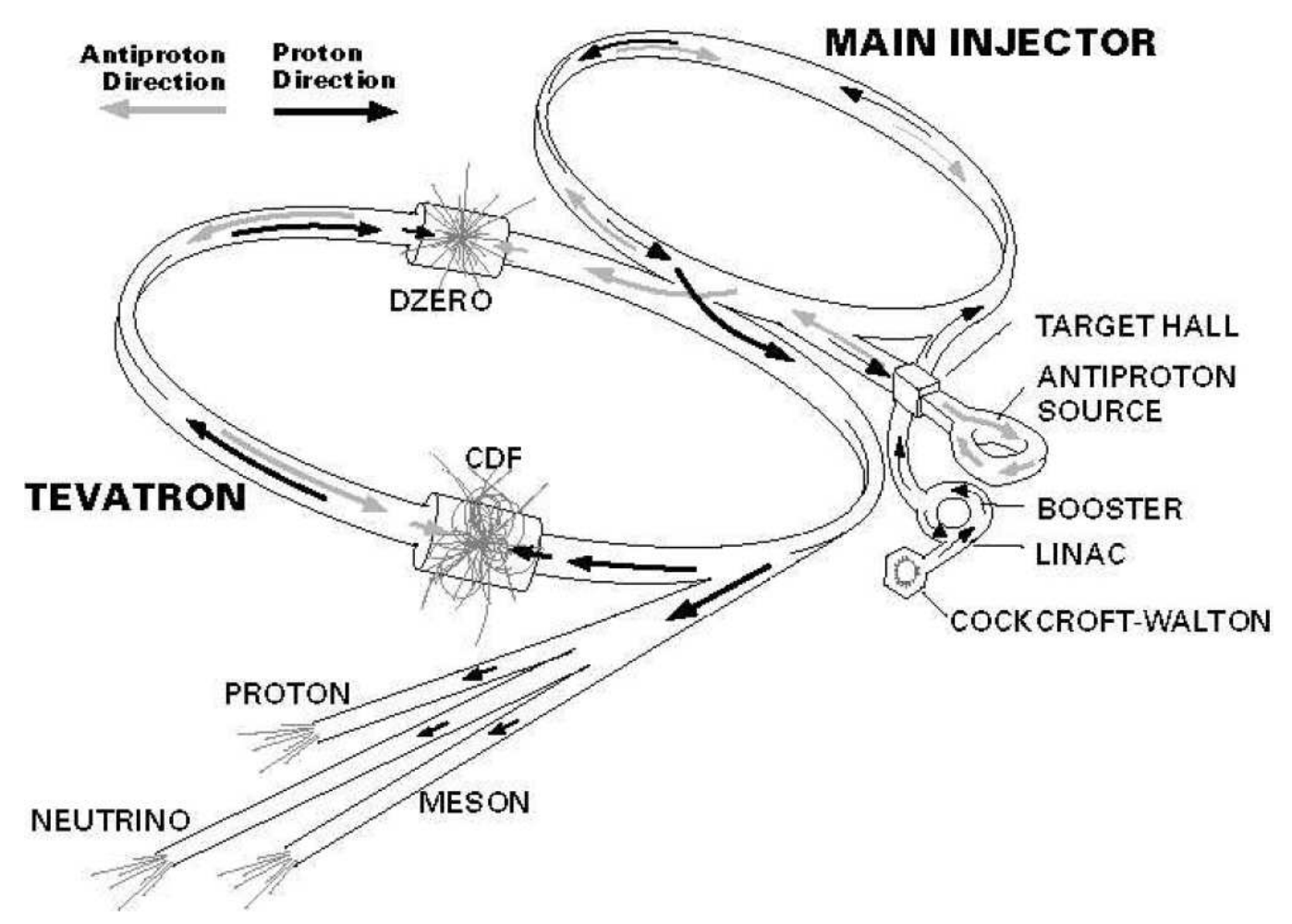

Figure 6. The schematic view of the accelerator chain at Fermilab. The Recycler is housed above the Main Injector. The Debuncher and Accumulator are in the Antiproton Source ring.

an oval shaped cathode surrounded by an anode with a uniform magnetic field passing through it. Cesium vapor coats the surface of the cathode allowing electrons to be pulled away from the cathode. The electric field strips the electrons from the hydrogen gas while the magnetic field causes the charged particles to move in helical paths. The $\mathrm{H}^{+}$ions accumulate on the cathode. Eventually, a charged particle will remove the $\mathrm{H}^{+}$ion from the cathode. Some of these $\mathrm{H}^{+}$ions release two electrons from the cesium coated surface. The $\mathrm{H}^{-}$ions migrate out of the enclosing anode and are accelerated by a $18 \mathrm{keV}$ extractor plate. The accelerated particles 
are passed through a bending magnet which extracts the $\mathrm{H}^{-}$ions. The $18 \mathrm{keV}$ hydrogen ion beam then enters the Cockcroft-Walton. The Cockcroft-Walton uses static electric fields to accelerate the ions. The electric fields are generated by charging capacitors in parallel and discharging them in series. The $\mathrm{H}^{-}$ions leave the Cockcroft-Walton pre-accelerator after being accelerated to $750 \mathrm{keV}$. Before entering the next accelerator, the Linac, the $80 \mu s$ continuous beam is subdivided into a $201.24 \mathrm{MHz}$ "bunched" beam using an electrostatic chopper and a single gap radio frequency $(\mathrm{RF})$ cavity.

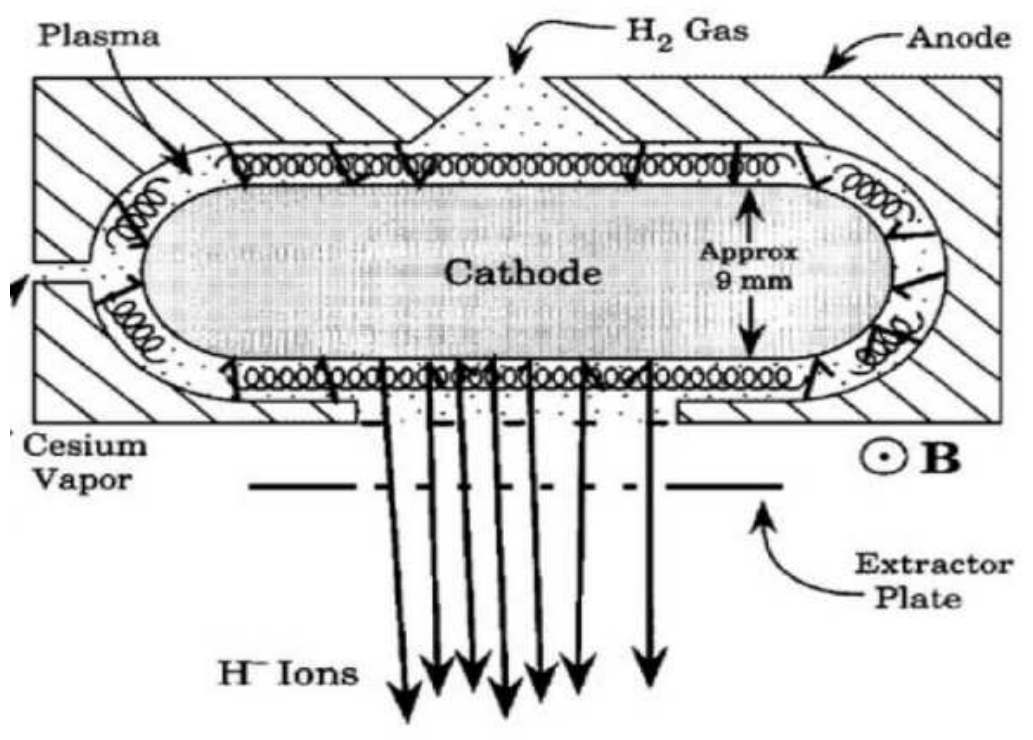

Figure 7. Hand drawing of the $\mathrm{H}^{-}$ion source or magnetron. 


\subsubsection{Linac}

The Linac is a linear accelerator broken into two sections: a series of $201.24 \mathrm{MHz} \mathrm{RF}$ accelerating cavities, (Figure 8), and a series of $804.96 \mathrm{MHz}$ RF accelerating cavities. As the beam passes through a series of RF cavities, the resonant cells become progressively longer. Each RF cavity has a resonant RF of $201.24 \mathrm{MHz}$ or $804.96 \mathrm{MHz}$ and contains a drift tube. The beam stays together via alternating focusing and defocusing quadrapole magnets embedded within the drift tubes. The drift tubes shield the $\mathrm{H}^{-}$ions from the decelerating electromagnetic field. Between the drift tubes, the $\mathrm{H}^{-}$ions are accelerated. The $\mathrm{H}^{-}$ions then leave the Linac at energies of $400 \mathrm{MeV}$ and are transfered to the Booster.

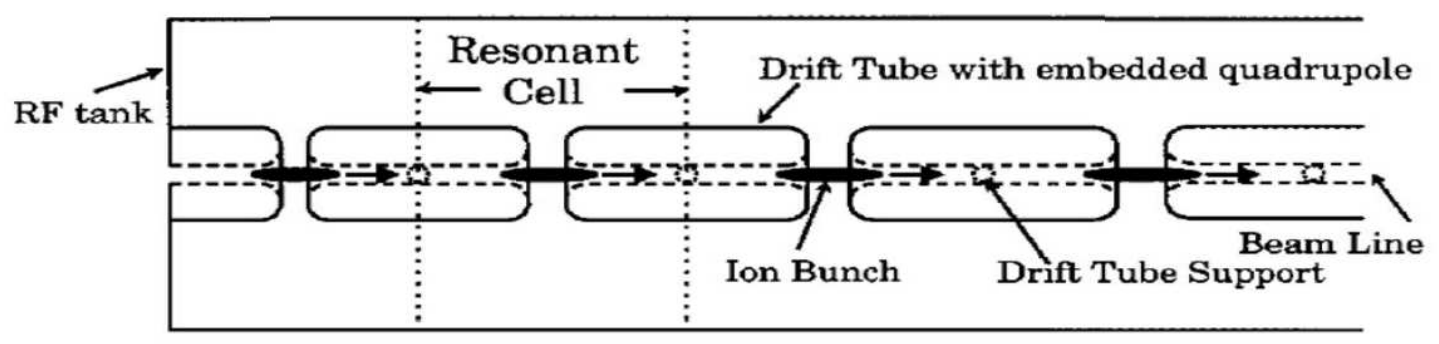

Figure 8. A drawing of the Linac RF cavity. 


\subsubsection{Booster}

The Booster is the first synchrotron accelerator in the Fermilab accelerator complex. The Booster operates in three modes: fill, accelerate, and dump. During the fill mode, the $\mathrm{H}^{-}$ions pass through a RF debuncher which keeps the beam energy fixed at $400 \mathrm{MeV}$ while reducing the momentum spread and removing the $804.96 \mathrm{MHz}$ RF structure. The incoming $\mathrm{H}^{-}$ions are brought into close proximity to the protons already circulating in the Booster ring. The protons are deflected from their orbital paths during filling just before a straight section in the beam line. Two opposite polarity dipole magnets merge the $\mathrm{H}^{-}$and the $\mathrm{H}^{+}$ions into a single beam. The combined beam is then passed through a carbon foil to strip the electrons away from the $\mathrm{H}^{-}$ions. The carbon foil causes some beam loss due to scattering. The resulting proton beam is deflected back to the proper orbital path. After accumulating $\sim 3 \times 10^{12}$ protons in the Booster, the fill process stops, and the magnets which deflected the protons from their orbit are turned off. The beam is then accelerated to $8 \mathrm{GeV}$ by increasing the magnetic fields to maintain the protons at the same radius, and increasing the RF. After acceleration, the Booster switches to dump mode and bunched protons are sent to the Main Injector.

\subsubsection{Main Injector}

The Main Injector is the second synchrotron accelerator in the chain of accelerators. The Main Injector receives protons from the Booster and performs the following tasks:

- acceleration of $8 \mathrm{GeV}$ protons from the Booster to $120 \mathrm{GeV}$ and delivery to the antiproton source; 
- acceleration of $8 \mathrm{GeV}$ protons from the Booster to $120 \mathrm{GeV}$ and delivery to the fixed target lines;

- acceleration of $8 \mathrm{GeV}$ protons from the Booster to $150 \mathrm{GeV}$ and delivery to the Tevatron;

- acceleration of $8 \mathrm{GeV}$ antiprotons from the Accumulator to $150 \mathrm{GeV}$ and delivery to the Tevatron;

- acceleration of $8 \mathrm{GeV}$ antiprotons from the Recycler to $150 \mathrm{GeV}$ and delivery to the Tevatron.

\subsubsection{Antiproton Source}

The antiproton source has three components: the fixed target; the Debuncher; and the Accumulator. $120 \mathrm{GeV}$ protons from the Main Injector strike the nickel target (Figure 9) and are collected using a lithium lens (Figure 10) and a dipole magnet for momentum selection. The limited ability to capture $8 \mathrm{GeV}$ antiprotons inside a small acceptance area yields only 12-24 antiprotons per million protons which hit the nickel target. Since the antiproton generation efficiency is so low, it takes more than twelve hours to generate enough antiprotons to begin $p \bar{p}$ collisions. Using a process called stochastic cooling, the transverse and longitudinal dispersion of the $8 \mathrm{GeV}$ antiprotons is minimized in the Debuncher before the antiprotons are transfered to the Accumulator. The Accumulator stores antiprotons until enough antiprotons have been collected to either start high energy physics collisions or to transfer the antiprotons to the Recycler. The Accumulator uses transverse and longitudinal adjustment techniques to minimize the dispersion while more antiprotons are stored. 


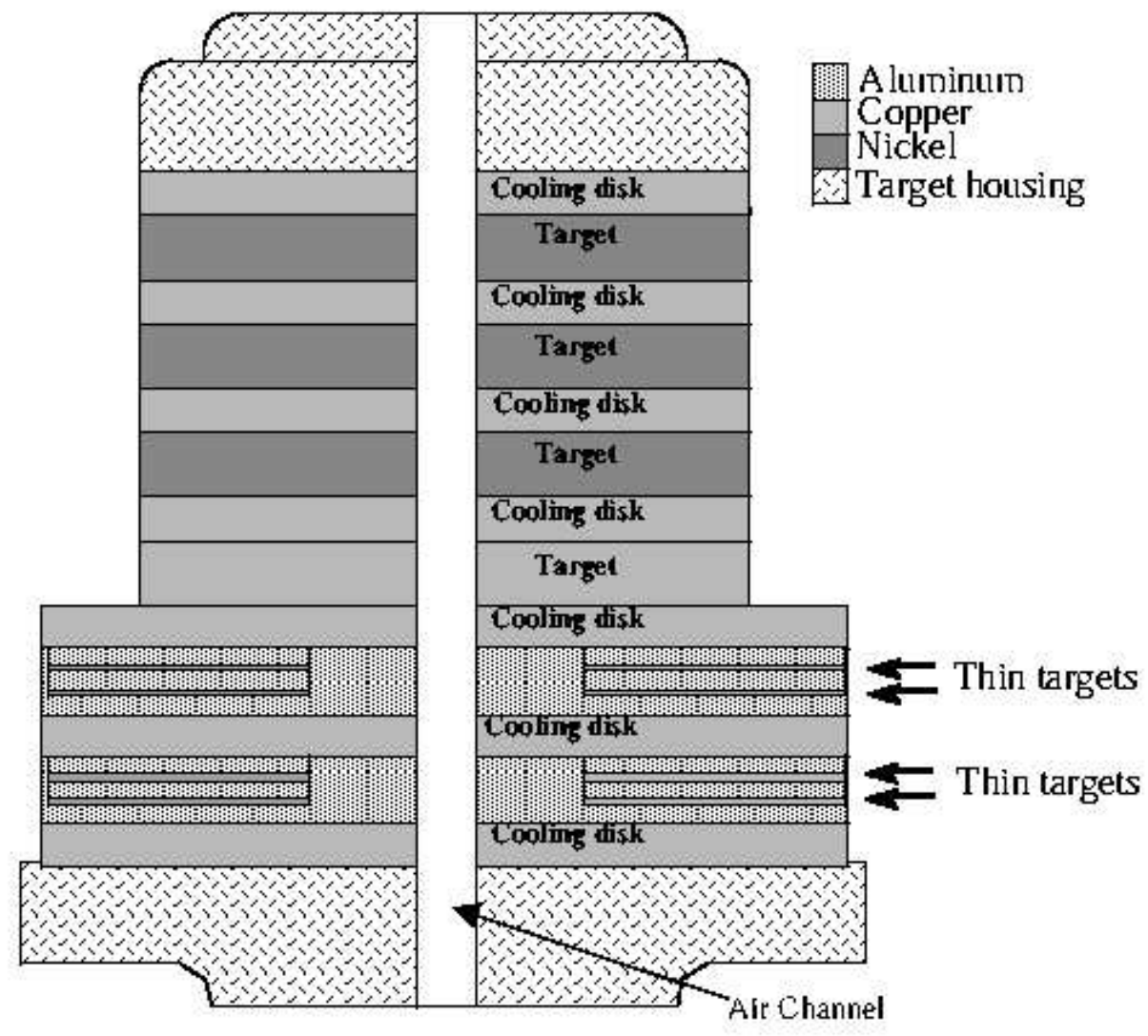

Figure 9. A schematic of the antiproton generating nickel target.

\subsubsection{Recycler}

The Recycler is an antiproton storage ring located above the Main Injector designed to operate in two ways: holding of $8 \mathrm{GeV}$ antiprotons from the Accumulator or storing of the remnants of a colliding beam. The efficiency of the Accumulator to accept more antiprotons from the Debuncher decreases as the number of antiprotons stored in the Accumulator increases. 


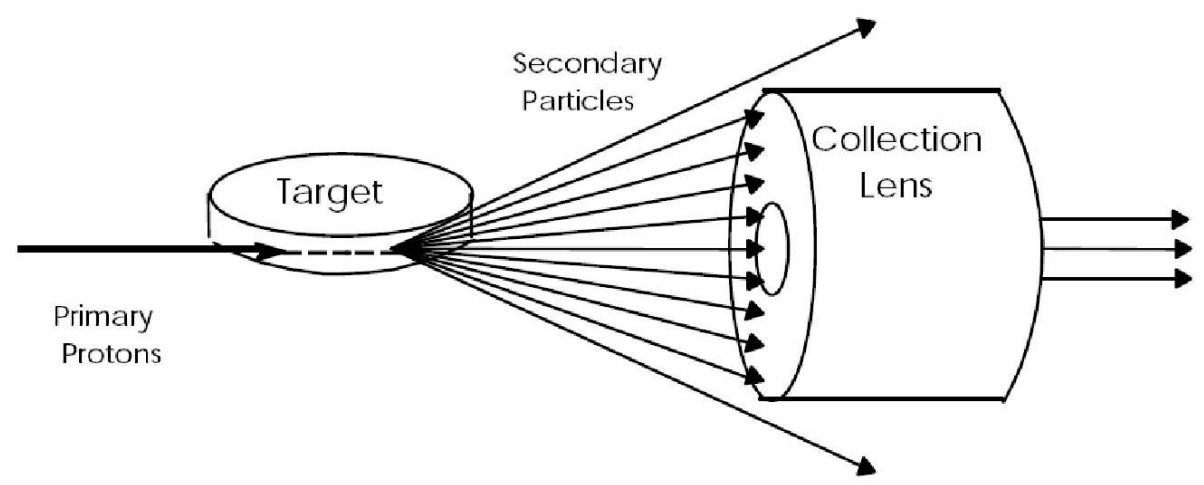

Figure 10. A diagram showing the nickel target and lithium lens.

\subsubsection{Tevatron}

The Tevatron is a synchrotron accelerator which uses superconducting magnets (up to $\sim 4$ T) to generate magnetic fields strong enough to allow the RF cavities to accelerate the protons and antiprotons to $980 \mathrm{GeV}$ within a $1 \mathrm{~km}$ radius circular ring.

The Main Injector sends 36 bunches of protons into the Tevatron, one bunch at a time. After all the protons have been loaded into the Tevatron, the Main Injector sends 36 bunches of antiprotons into the Tevatron, four bunches at a time. The two beams follow the same orbital paths, but in opposite directions, and the beams do not collide since they are forced to travel in helical paths. The 36 bunches are separated into three super bunches separated by $2.64 \mu s$. Within a super bunch, the twelve bunches are separated by $396 \mathrm{~ns}$.

The two beams are magnetically squeezed and forced to pass through each other at two separate interaction points were the $\mathrm{CDF}$ and $\mathrm{D} \varnothing$ detectors are located. A store is defined as 
the period of time in which the two beams are colliding. A typical store lasts twenty-four hours or more based on the initial density per bunch of antiprotons in the accelerator.

\subsection{The DØ Detector}

The D $\varnothing$ detector $(22)$ was built to study high mass states and large $p_{T}$ phenomena. The D detector is built using a variety of particle detectors installed in layers as shown in Figure 11. The inner most layer includes the track finding detectors for charged particles surrounded by a sampling calorimeter, which is surrounded by a muon spectrometer. The central tracking system starts with a silicon microstrip tracker, a central scintillating fiber tracker, and a solenoid producing an internal $2 \mathrm{~T}$ magnetic field. A preshower scintillating fiber detector is located between the solenoid magnet and the calorimeter. Scintillating tile luminosity planes surround the beam pipe at two ends of the detector. The sampling calorimeter is segmented into three separate detectors (one central and two end detectors). The three calorimeter detectors have absorbers made of depleted uranium, copper, and stainless steel. Liquid argon is the active medium inside the calorimeters. The muon detector system has a $1.8 \mathrm{~T}$ toroid with scintillator counters, proportion drift tubes, and mini drift tubes.

\subsubsection{The DØ Coordinate System}

The D $\varnothing$ coordinate system uses a mix of cylindrical coordinates and spherical coordinates. There are three variables as shown in Figure $12(\mathrm{r}, \phi$, and $\theta)$. The $\mathrm{z}$ axis is taken along the beam pipe in the direction of the protons. $\phi$ and $\theta$ have the same meaning as in the spherical

coordinate system but $\mathrm{r}$ is defined by the cylindrical coordinates system. $\mathrm{r}, \phi$, and $\theta$ in terms of $\mathrm{x}, \mathrm{y}$, and $\mathrm{z}$ are defined as: 


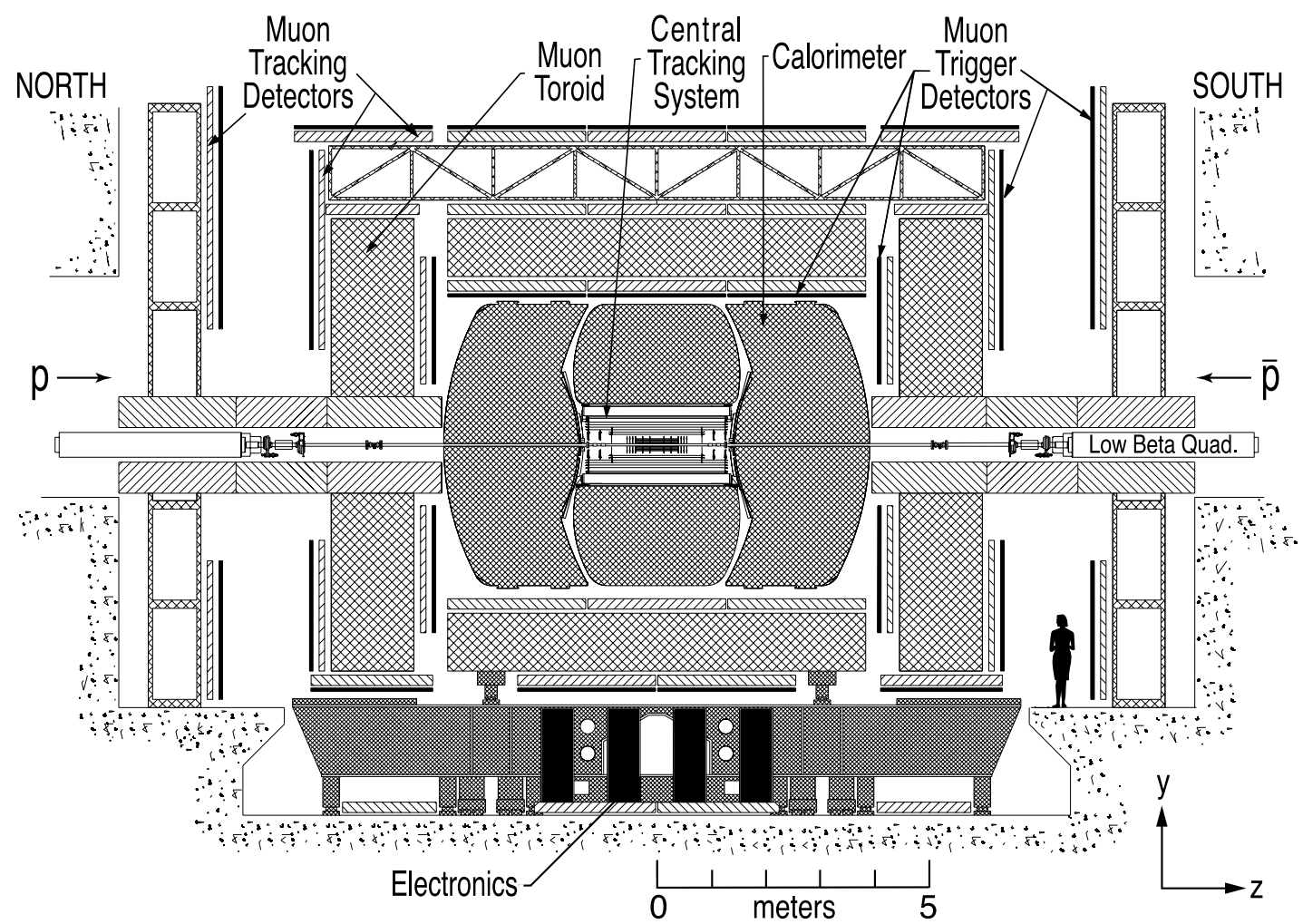

Figure 11. Side view of the $\mathrm{D} \varnothing$ detector.

$$
\begin{gathered}
r^{2}=x^{2}+y^{2} ; \\
\phi=\arctan \left(\frac{y}{x}\right) ; \\
\theta=\arctan \left(\frac{\left(x^{2}+y^{2}\right)^{\frac{1}{2}}}{z}\right) .
\end{gathered}
$$




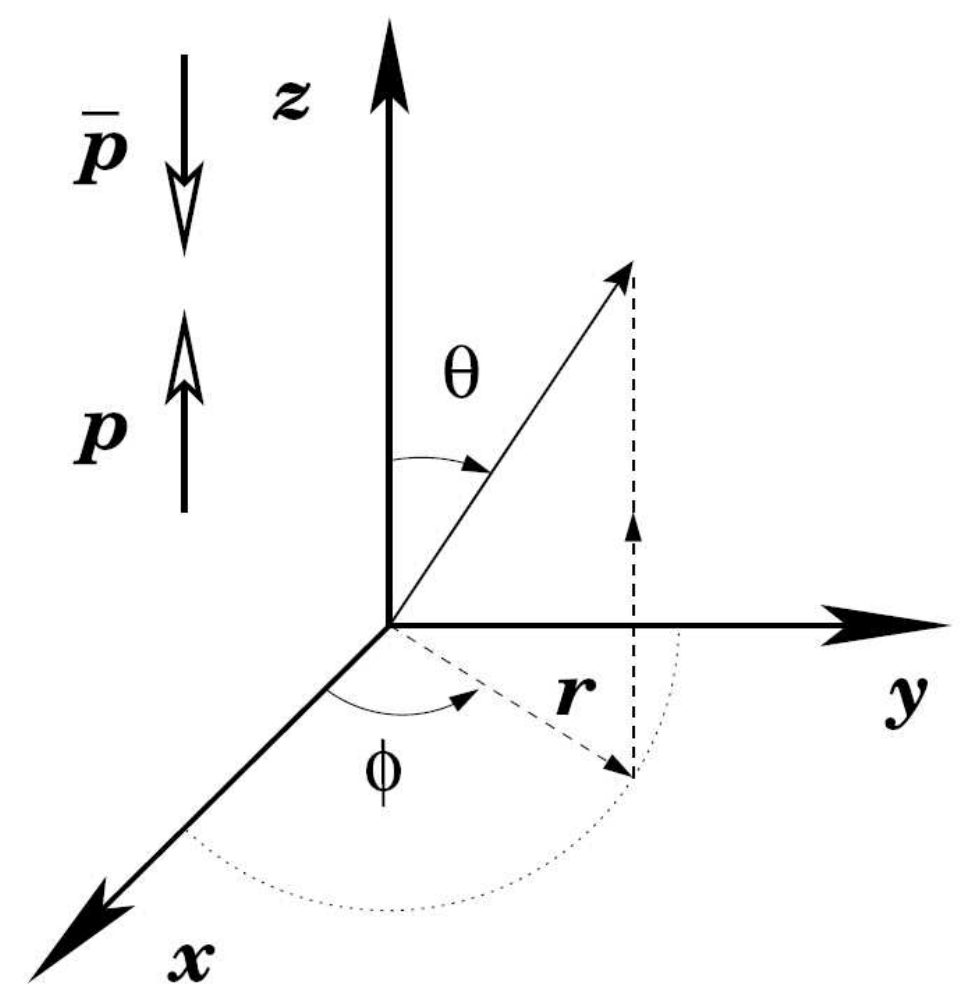

Figure 12. The D $\varnothing$ detector coordinate system.

Instead of using the scattering angle $\theta$, pseudorapidity, $\eta$, is used which has the property that the number of particles produced in a given range of $\eta$ is invariant with respect to boosts along the $\mathrm{z}$ direction when the mass of the particles is zero. If the mass of the particles is not equal to zero then the proper angle to use is rapidity, y. y, $\eta$, and $\theta$ are defined as:

$$
y=\frac{1}{2} \ln \left(\frac{E+p_{z}}{E-p_{z}}\right) \quad y=\eta \text { when } m=0 \text { or } \frac{m}{E} \rightarrow 0
$$




$$
\begin{gathered}
\eta=-\ln \left(\tan \left(\frac{\theta}{2}\right)\right)=\operatorname{arccosh}\left(\frac{1}{\sin (\theta)}\right) \\
\theta=2 \arctan \left(e^{-\eta}\right)=\arcsin \left(\frac{1}{\cosh (\eta)}\right) .
\end{gathered}
$$

The energy and momentum of a particle using rapidity is given by:

$$
E=m_{T} \cosh (y)
$$

$$
p_{z}=m_{T} \sinh (y)
$$

where the transverse mass, $m_{T}$, is defined as:

$$
m_{T}^{2}=m^{2}+p_{x}^{2}+p_{y}^{2}
$$

The transverse momentum is the projection of the momentum vector to the $(\mathrm{x}, \mathrm{y})$ plane:

$$
p_{T}=p \sin (\theta)=\frac{p}{\cosh (\eta)}
$$

The limit of $p_{T} \rightarrow E_{T}$ as $m \rightarrow 0$ :

$$
E_{T}=E \sin (\theta)=\frac{E}{\cosh (\eta)}
$$


Finally $p_{x}, p_{y}$, and $p_{z}$ are calculated using $\mathrm{p}, \phi$, and $\eta$ via:

$$
\begin{gathered}
p_{x}=p_{T} \cos (\phi)=\frac{p}{\cosh (\eta)} \cos (\phi) ; \\
p_{y}=p_{T} \sin (\phi)=\frac{p}{\cosh (\eta)} \sin (\phi) ; \\
p_{z}=p \cos (\theta)=p \cos \left(\arcsin \left(\frac{1}{\cosh (\eta)}\right)\right) .
\end{gathered}
$$

\subsubsection{Luminosity Monitor}

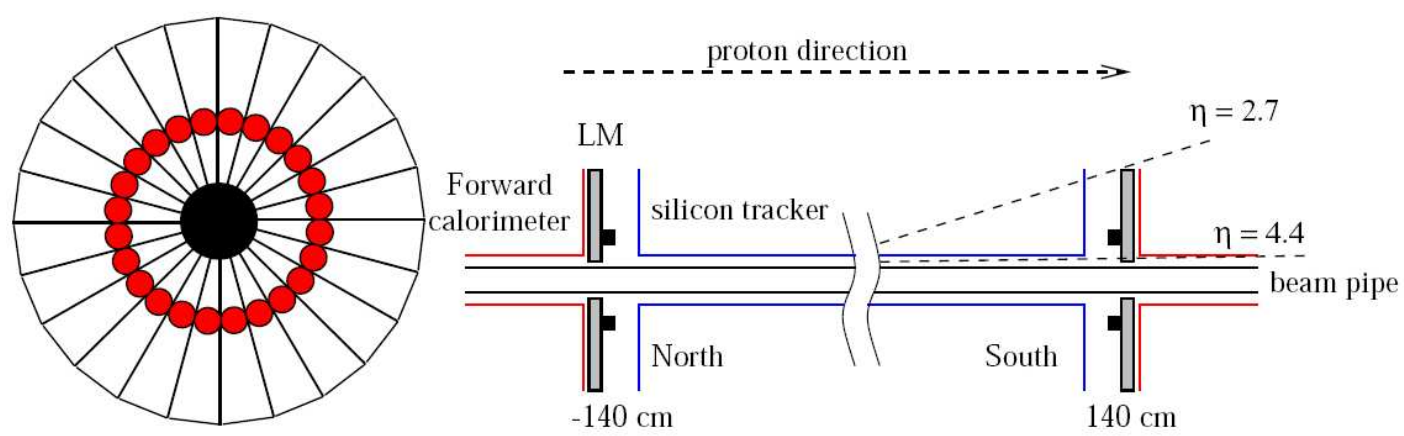

Figure 13. A diagram of the D $\varnothing$ luminosity monitors. The $r-\phi$ view is shown on the left, while the $\mathrm{r}-\mathrm{z}$ view of the two monitors is shown on the right. The photomultiplier tubes are represented by small circles on each tile. 


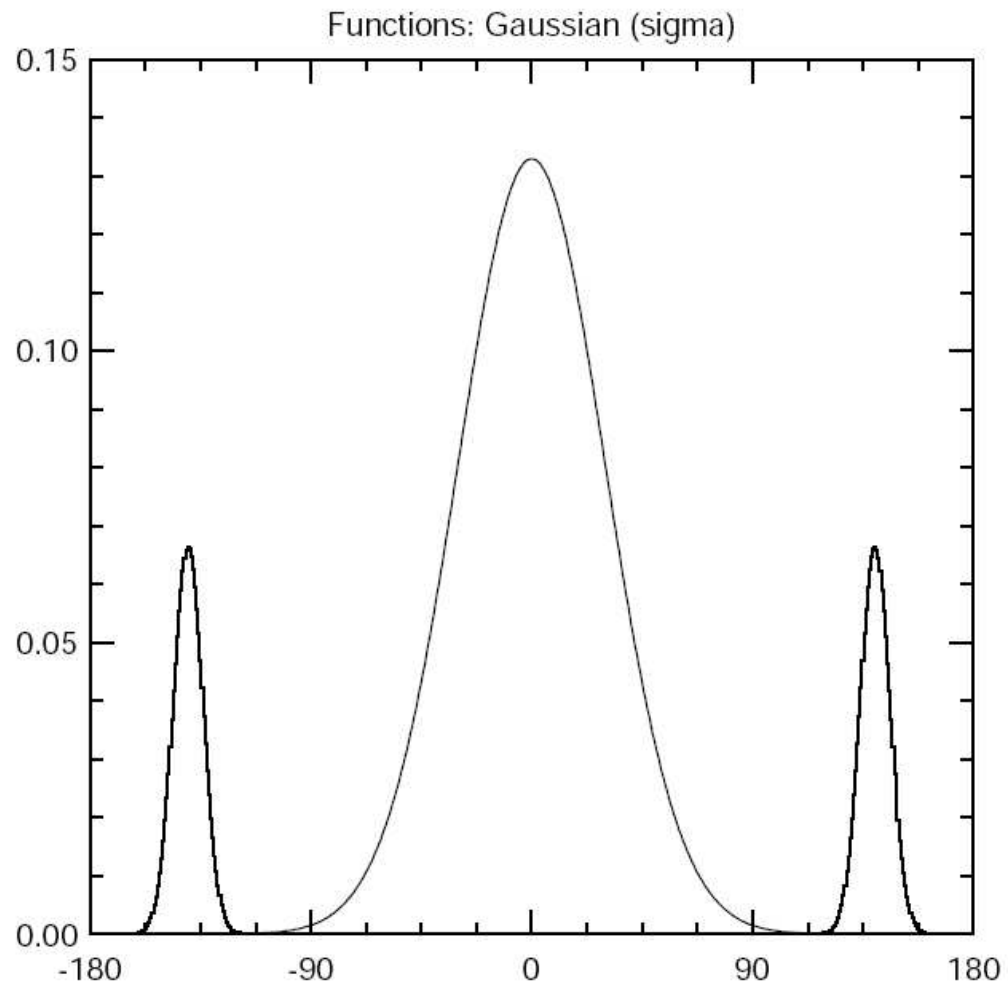

Figure 14. The z vertex distribution from inelastic $p \bar{p}$ collisions.

The number of $p \bar{p}$ collisions, over a given time and area, is calculated by the luminosity detector (23). The two luminosity detectors are located at $\pm 140 \mathrm{~cm}$ from the center of the $\mathrm{D} \varnothing$ detector along the $\mathrm{z}$ direction. The luminosity detectors cover the $\eta$ range of $2.7<|\eta|<$ 4.4 in a $\phi$ ring (Figure 13). Each detector consists of twenty-four wedge shaped scintillating tiles which are $15 \mathrm{~cm}$ long and $1.6 \mathrm{~cm}$ thick. The scintillating tiles are read out by high gain photomultiplier tubes attached perpendicularly to each tile. Using the time of flight equation $z_{v}=\frac{c}{2}\left(t_{-}-t_{+}\right)$, where $t_{-}$and $t_{+}$are the times when the decay products hit the downstream 
and upstream luminosity monitors respectively, a quick location of the z position of the primary vertex is determined, as shown in Figure 14. Only events with $\left|z_{v}\right|<100 \mathrm{~cm}$ are used to count the number of $p \bar{p}$ collisions. The instantaneous luminosity is a measure of the particle flux per unit area and per unit time $\left(\mathrm{cm}^{-2} \mathrm{~s}^{-1}\right)$, and is defined as:

$$
L=\frac{f \bar{N}_{L M}}{\sigma_{L M}}
$$

$\mathrm{f}$ is the beam crossing frequency, $\bar{N}_{L M}$ is the average number of $p \bar{p}$ inelastic collisions for one rotation, and $\sigma_{L M}$ is the effective cross section (taking into account the acceptance and efficiency of the luminosity monitor). The effective cross section is the measure of the interaction probability per unit flux ${ }^{1}$. The luminosity is averaged and reported in sixty second increments luminosity block numbers (LBN). Figure 15 shows the total luminosity delivered by the Tevatron and recorded with the $\mathrm{D} \varnothing$ detector, covering the data taking period of April 2002 to October 2006. This analysis used the data from the period of April 2002 to August 2004.

\footnotetext{
${ }^{1}$ Cross section are usually expressed in barns, where 1 barn $=10^{-24} \mathrm{~cm}^{2}$. Integrated luminosity is expressed in $b^{-1}$.
} 


\section{B. Run II Integrated Luminosity 19 April 2002 - 31 october 2006}

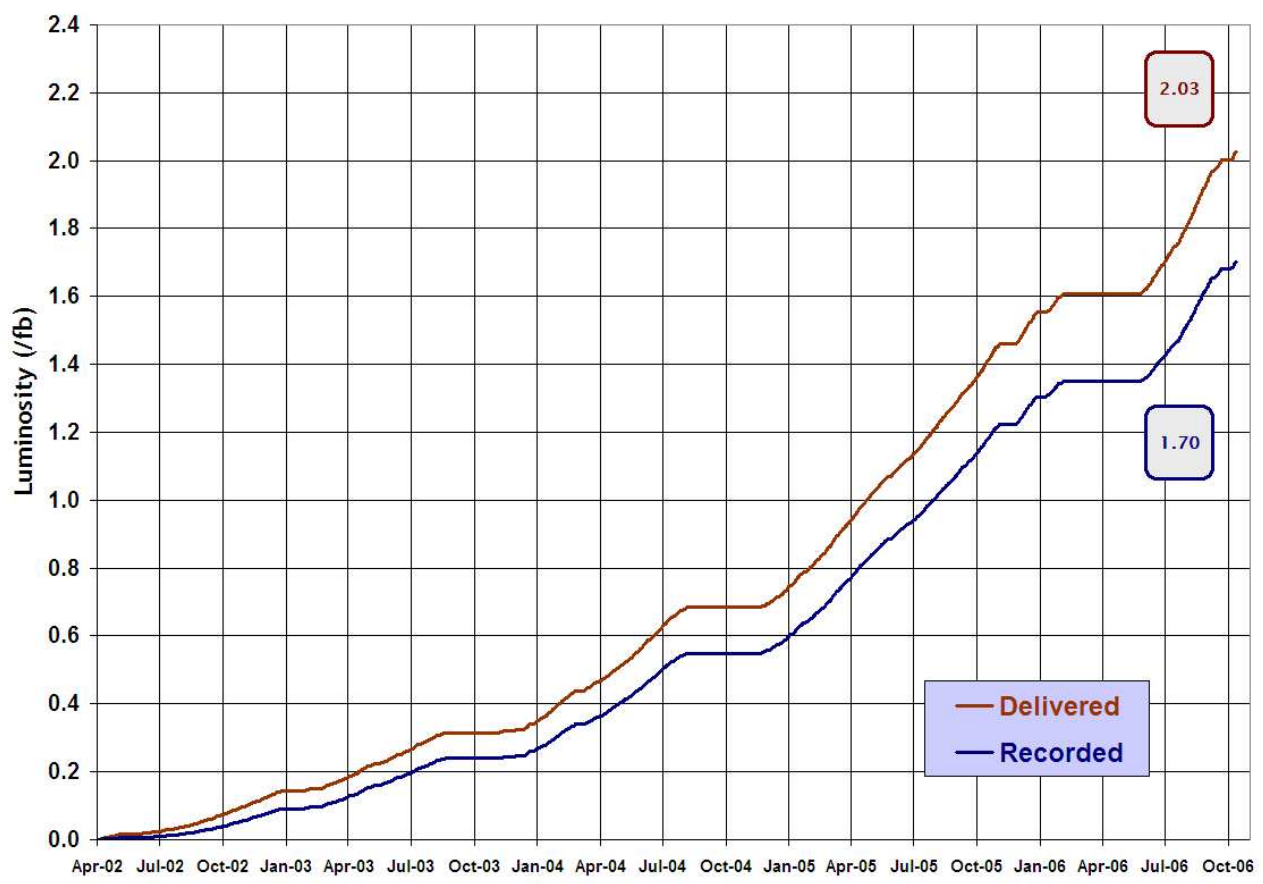

Figure 15. The total amount of luminosity delivered to and recorded by the $\mathrm{D} \varnothing$ detector. 


\subsubsection{The Central Tracking System}

The central tracking system (Figure 16) measures the sign of the charge, the position, and the momentum of charged particles transversing the following set of detectors:

- the 2 Tesla solenoid;

- the silicon microstrip tracker;

- the central fiber tracker.

The central tracking system depends on the Lorentz force $(\vec{F}=q \vec{v} \times \vec{B})$ to bend the path of particles with charge (q) based on the strength of the magnetic field $(\vec{B})$ and velocity $(\vec{v})$ of the particles. The solenoid generates a uniform $2 \mathrm{~T}$ magnetic field along the $\mathrm{z}$ direction causing the charged particles to travel in helical paths characterized by the radius of curvature $r=\frac{p_{T}}{q B}$ and $p_{z}$. The charge of a particle is measured by the direction of the deflection. The momentum is measured by the curvature of the charged particle. The charged particles deposit small amounts of ionization energy into the silicon and scintillating fibers. A collection of small energy deposits (hits) are used to reconstruct the path or track of the particles.

\subsubsection{The Solenoid}

The superconducting solenoid is based on superconducting $\mathrm{Cu}$ NbTi multi-filamentary cable strands which are stabilized by aluminum. The solenoid is $2.73 \mathrm{~m}$ in length and $1.42 \mathrm{~m}$ in diameter. The $2 \mathrm{~T}$ magnetic field is generated using a current of $4749 \mathrm{~A}$, and cooled using liquid Helium at 4.6 K. Figure 17 shows the magnetic field lines from the solenoid. The polarity 


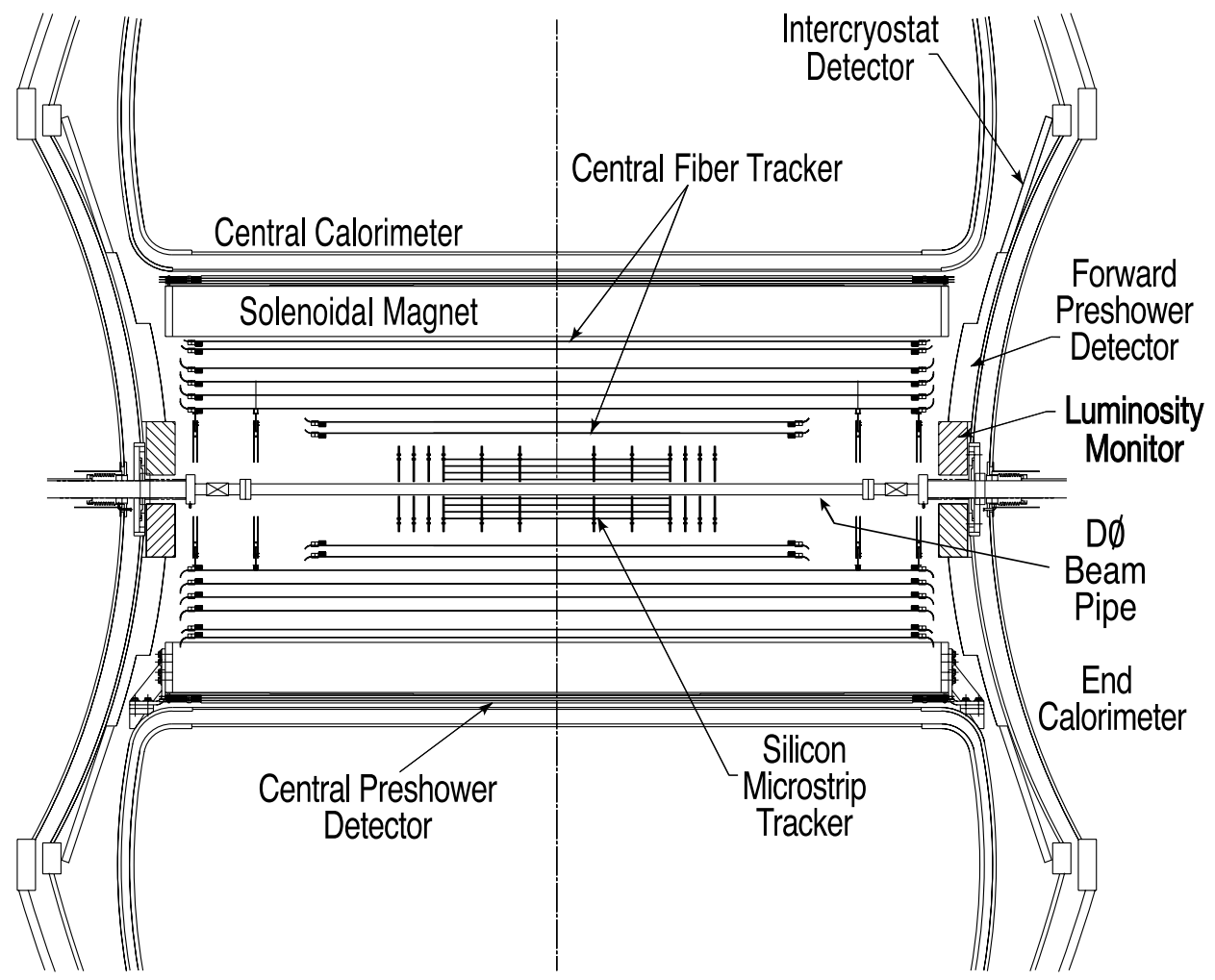

Figure 16. Side view of the Central Tracking detectors.

of the magnetic field is reversed periodically, but remains constant during a period of data taking (a store).

\subsubsection{The Silicon Microstrip Tracker Detector}

The silicon microstrip tracker (SMT) (24) is located just outside of the beryllium beam pipe, as shown in Figure 18. The SMT allows for track finding and vertex identification out to $|\eta|<3.0$. The tracker consists of doped silicon to produce $\mathrm{p}-\mathrm{n}$ junction diodes operating at reverse bias. The p-n junctions form a depleted zone where there are no electrons in the 


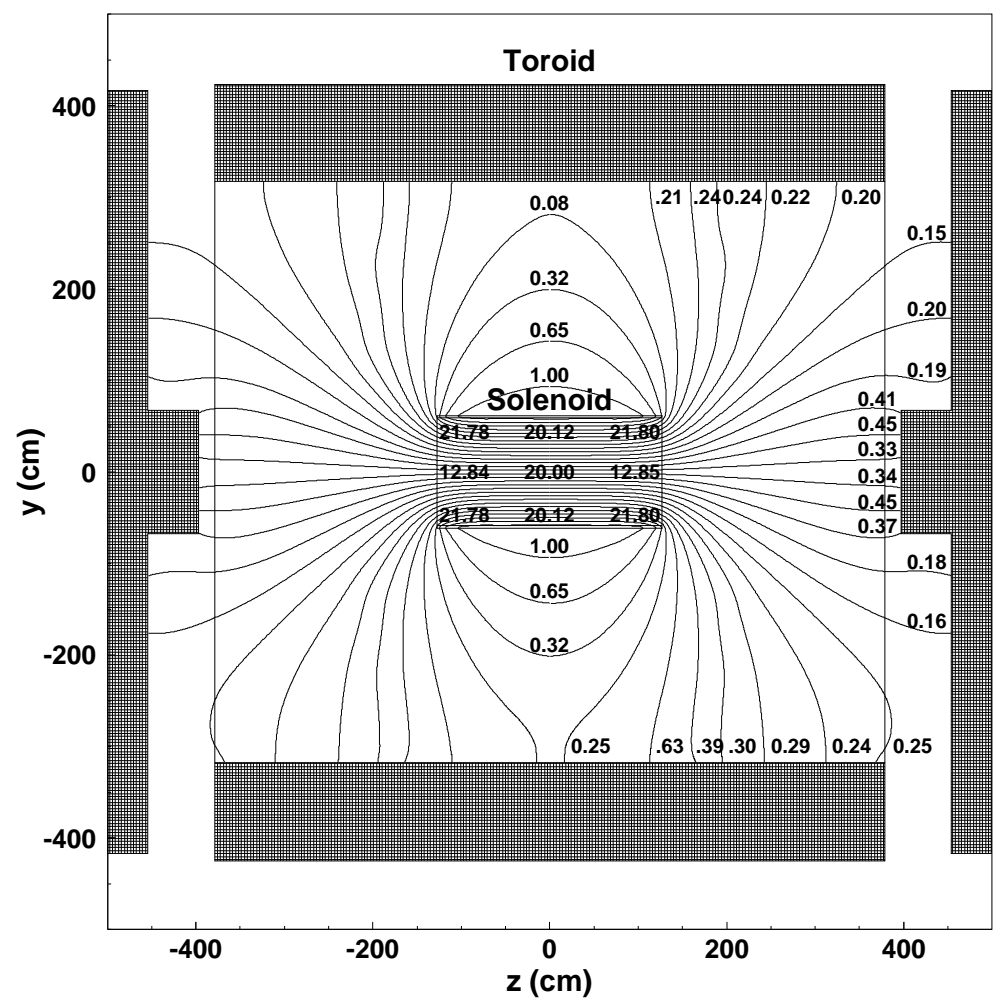

Figure 17. The magnetic field lines extended out to the magnetic toroid. The strength of the magnetic field is given in kiloGauss $(10 \mathrm{kG}=1 \mathrm{~T})$

conduction band. An applied reverse bias enhances the electric field across the depleted zone. Charged particles transversing the silicon will create electron hole pairs in the depleted zone which will be collected and produce a charge pulse. The charge is stored in capacitors and then read out and digitized. The SMT provides a precise measurement of the trajectory of charged particles. The axial hit resolution is $10 \mu \mathrm{m}$ and the $\mathrm{z}$ hit resolution (for barrels) can be within $35 \mu \mathrm{m}$. 


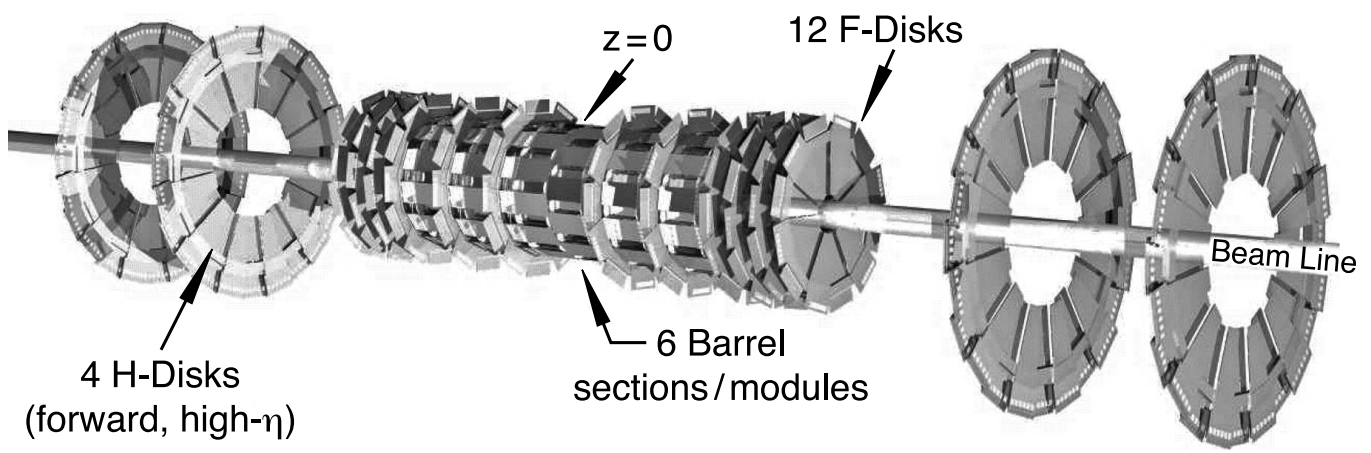

Figure 18. A 3D layout of the Silicon Mircostrip Tracker.

The silicon wafers are formed into disks and barrels. The barrels are cylinders encompassing the beam pipe and measure the $(\mathrm{r}, \phi)$ coordinates of charged particle, but have a large uncertainty in the $\mathrm{z}$ direction. The disks are perpendicular to the beam pipe and can measure the $(\mathrm{r}, \phi$, and $\mathrm{z})$ coordinates of the trajectory of a charged particle. There are six barrels centered symmetrically around $\mathrm{z}=0$. At the end of each barrel there is an $\mathrm{F}$ disk. Each barrel consists of four readout layers, and each readout layer contains two staggered and overlapping sublayers, as shown in Figure 19. The F disks contain twelve double-sided wedge detectors (Figure 20). The third barrel is capped off with a F disk followed by three more F disks. Following the three F disks, there are two large diameter $\mathrm{H}$ disks which contain twenty-four pairs of single-sided detectors glued back to back. 3.2.3.2 shows the $|z|$ location of the center of each silicon detector. 3.2.3.2 shows the radii of the silicon detector components from the center of the beam pipe. The silicon microstrip tracker has a total of 792,567 readout channels. 


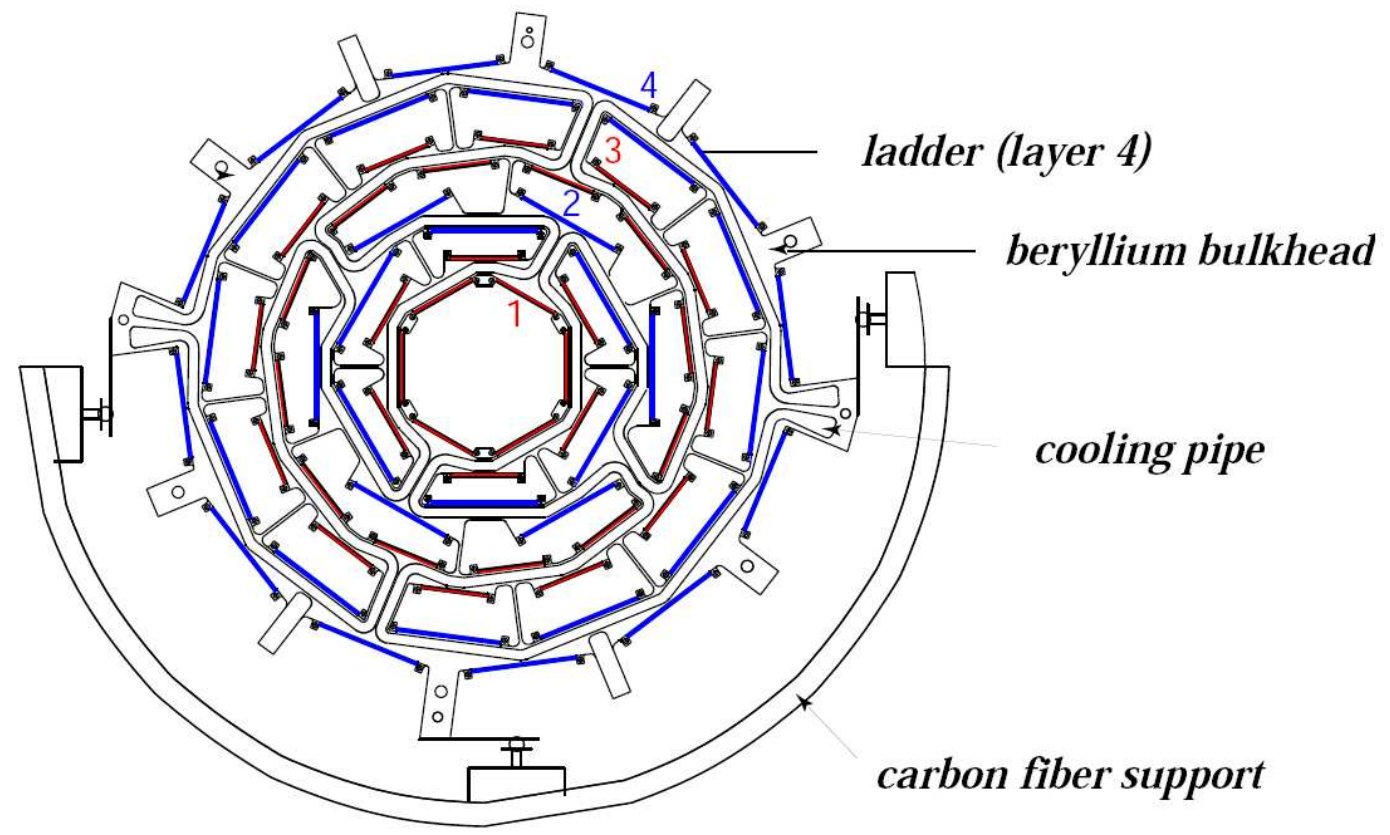

Figure 19. The r-phi view of the silicon barrels.

\begin{tabular}{|lc|}
\hline unit & $|z|$ location $(\mathrm{cm})$ \\
\hline Barrel & $6.2,19.0,31.8$ \\
Fdisk & $12.5,25.3,38.2,43.1,48.1,53.1$ \\
Hdisk & $100.4,121.0$ \\
\hline
\end{tabular}

TABLE III

LOCATION OF SILICON MICROSTRIP TRACKER COMPONENTS ALONG THE Z DIRECTION STARTING FROM THE CENTER OF THE DETECTOR. 


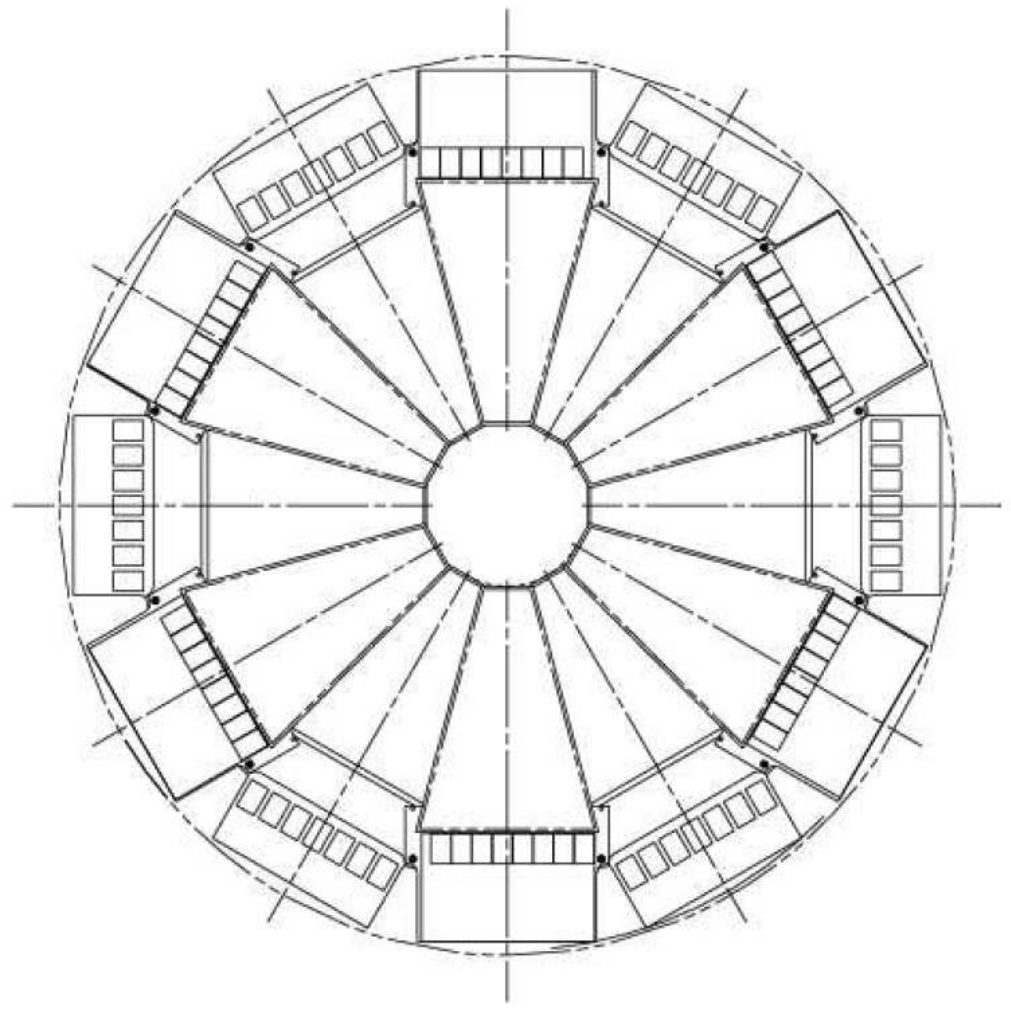

Figure 20. The r-phi view of the silicon wedge detectors of the F-disks.

\begin{tabular}{|lc|}
\hline unit & radius $(\mathrm{cm})$ \\
\hline 4 Barrels & $2.7,4.6,7.6,10.5$ \\
Fdisk & 2.6 to 10.0 \\
Hdisk & 9.5 to 26 \\
\hline
\end{tabular}

TABLE IV 


\subsubsection{The Central Fiber Tracker Detector}

The central fiber tracker detector (CFT) (25) measures the position of charged particles using scintillating fibers. The ionizing particles excite the atoms in the scintillating material which then release photons of light with wavelengths around $340 \mathrm{~nm}$. The scintillating fibers have a dye which absorbs $340 \mathrm{~nm}$ light and emits $530 \mathrm{~nm}$ light that is detected by the visible light photon counters (VLPC).

The pseudo-rapidity coverage of the CFT is $|\eta|<2.0$. The CFT has an inner radius of $19.5 \mathrm{~cm}$ and extends out to $51.5 \mathrm{~cm}$. It is composed of 76,800 scintillating fibers which form eight superlayers with increasing radius (Figure 21). Each superlayer is broken down into one axial layer (along the $\mathrm{z}$ axis) and one stereo layer $\left( \pm 3^{\circ}\right)$. Each axial and stereo layer is further broken down into two layers (Figure 22). The inner layer (closest to the beam pipe) lies in a drilled out molding which separates the fibers by 926-990 $\mu m$ (depending on the superlayer). The outer layer fibers fit on top of two adjacent inner layer fibers. Two out of the eight super layers are made of $166 \mathrm{~cm}$ long scintillating fibers while the other six superlayers are made out of $252 \mathrm{~cm}$ scintillating fibers. Each superlayer can identify the $\eta$ and $\phi$ position of a charged particle within $100 \mu \mathrm{m}$. Each scintillating fiber is $835 \mu \mathrm{m}$ thick. The light leaving the scintillating fibers enters clear fiber waveguides and is turned into charge via impurity band silicon avalanche photodetectors. The Level-1 and Level-2 triggers (described in Chapter 4) use hits from the axial layers. The Level-3 trigger uses axial and stereo information. 


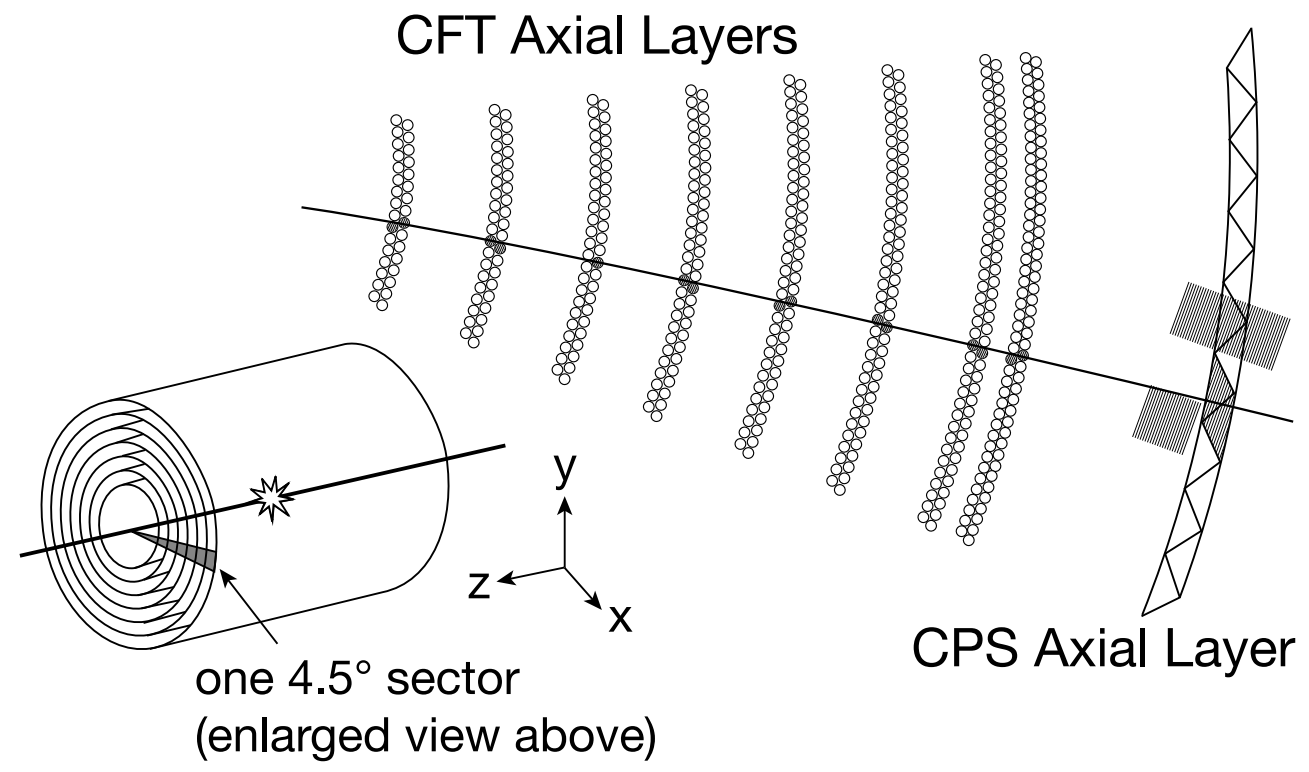

Figure 21. a) The Central Fiber Tracker located between the Silicon Microstrip Tracker and the Solenoid. b) The cross section blow up of the fibers from two layers. 


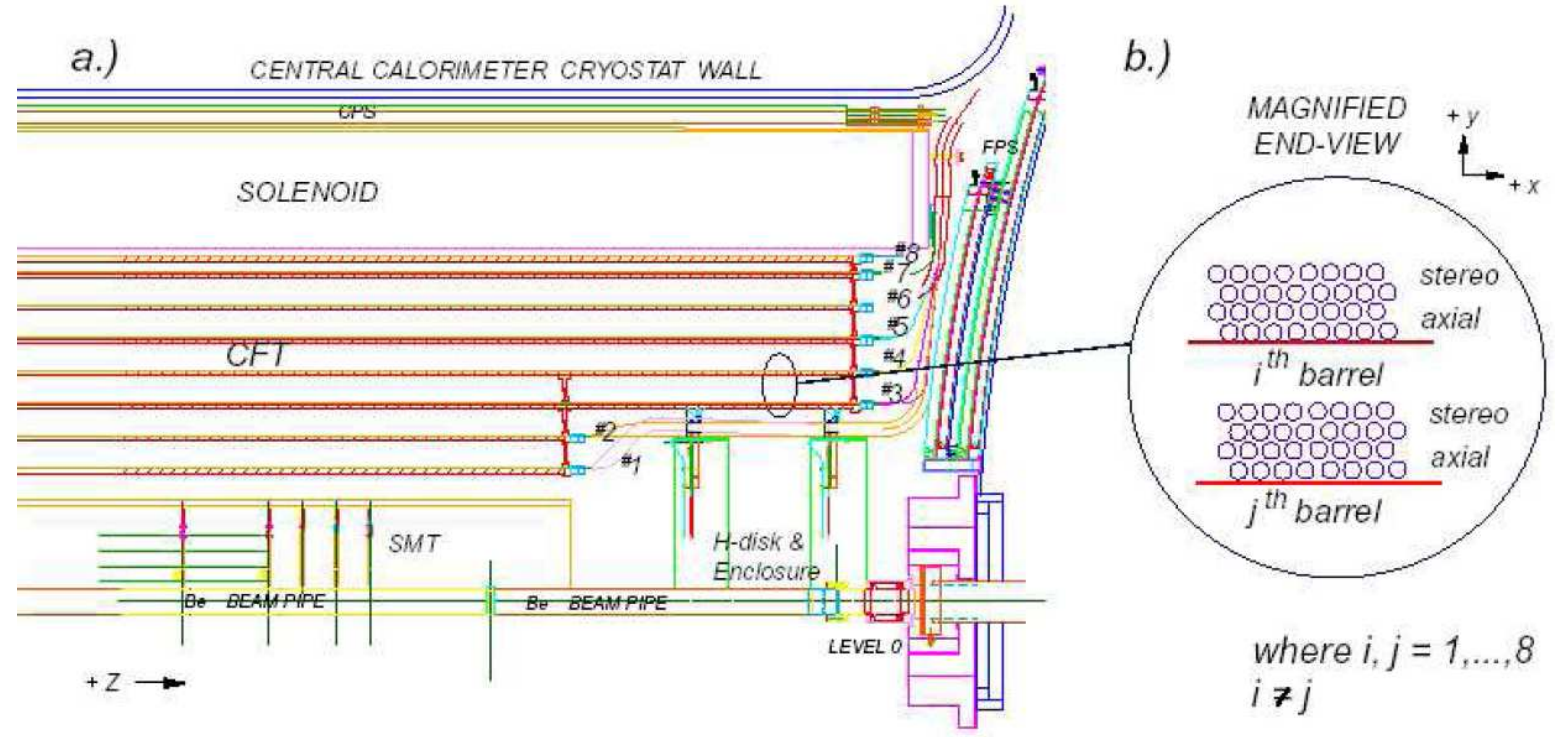

Figure 22. a) The r-z diagram of the Central Fiber Tracker. b) A cross section of view of the fibers. 


\subsubsection{The Preshower Detectors}

The scintillating fiber preshower detectors $(26 ; 27)$ are located in between the solenoid and the calorimeter, shown in Figure 23 and Figure 24. They are designed to improve the identification and energy measurement of electrons and photons. There are two preshower detectors: a central detector covering $|\eta|<1.3$ and two forward detectors covering $1.5<|\eta|$ $<2.5$. The scintillating fibers are triangular strips, individually wrapped in aluminized mylar, epoxied, and interlocked together as shown in Figure 25. This arranges scintillator layers without creating any dead space and thereby improves the accuracy of position measurements. The center of each strip contains a $835 \mu \mathrm{m}$ diameter wavelength shifting fiber which collects and carries the light to the clear fiber light-guide, which is connected to the VLPCs. The other end of the triangle strip is coated with silver. The visible light leaves the clear fiber waveguides and is turned into electric charge by a $2 \times 4$ array of $1 \mathrm{~mm}$ diameter pixel impurity band silicon avalanche photodetectors. There are 22,564 readout channels for the preshower detectors.

\subsubsection{Central Preshower}

The central preshower detector (CPS) is sandwiched between the solenoid and the central calorimeter and covers $|\eta|<1.3$. After the solenoid, there is a lead radiator (covered by a stainless steel skin) which has a thickness of one radiation length $\left(\mathrm{X}_{o}\right)^{1}$. The CPS is broken up into two separate units, north and south of $\mathrm{z}=0$. Each detector has three layers of triangular

\footnotetext{
${ }^{1}$ The mean distance over which a high-energy electron losses all but $1 / e$ of its energy by Bremsstrahlung, and equivalently it is the $\frac{7}{9}$ of the mean free path for pair production by high energy photons.
} 


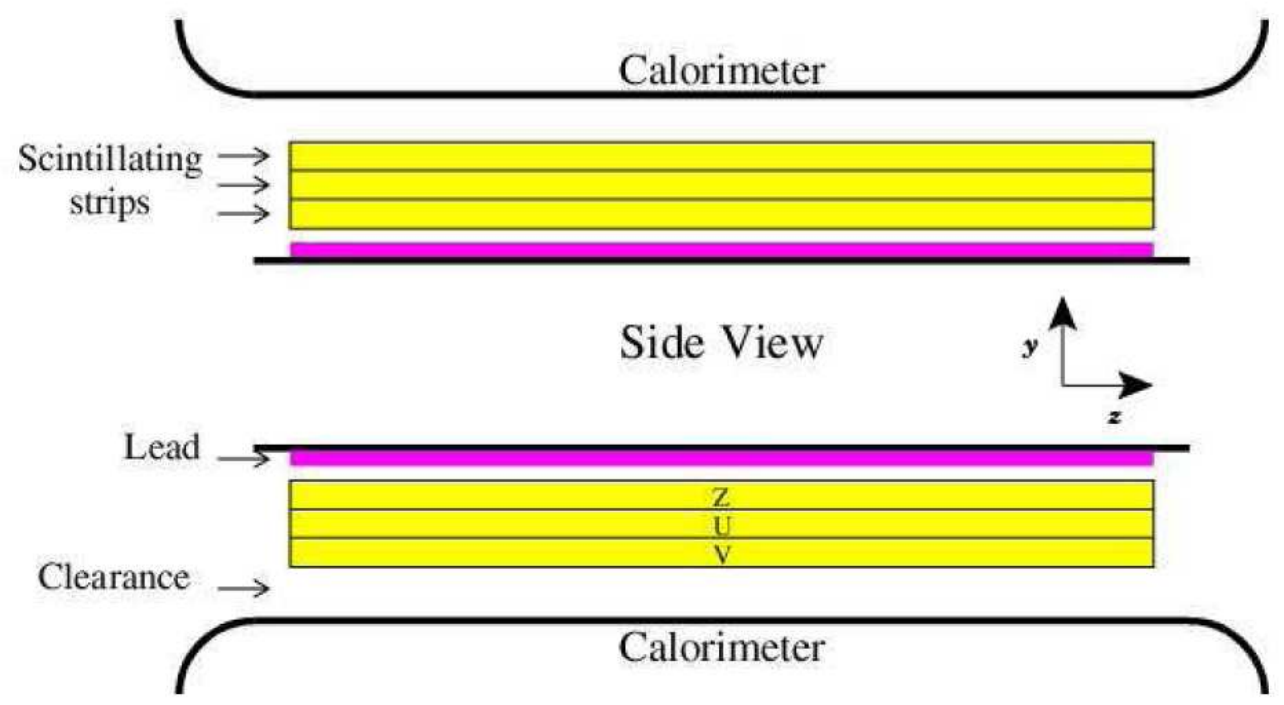

Figure 23. Schematic r-z side view of the central preshower detector.

shaped fibers. The inner most layer lies along the $\mathrm{z}$ axis, the next layer lies along the $\mathrm{z}$ axis at a stereo angle of $+23.8^{\circ}(\mathrm{u})$, and the final layer lies along the $\mathrm{z}$ axis at a stereo angle of $-24.0^{0}$ (v), as shown in Figure 26. Each of the three layers, axial, u, and v consist of 1280 strips. The central preshower detector is subdivided into $80 \phi$ sectors for Level-1 triggering, therefore 16 fibers (per layer) make up one $\phi$ sector. Only the axial layers are used for Level-1 triggering, but all three layers are used for Level-2 triggering.

\subsubsection{Forward Preshower}

The forward preshower detectors (FPS) are broken up into two units which are attached to the two end calorimeters covering $1.5<|\eta|<2.5$. There are four layers of triangular shaped scintillating strips in the detector which are broken up into two regions: minimum ionizing 


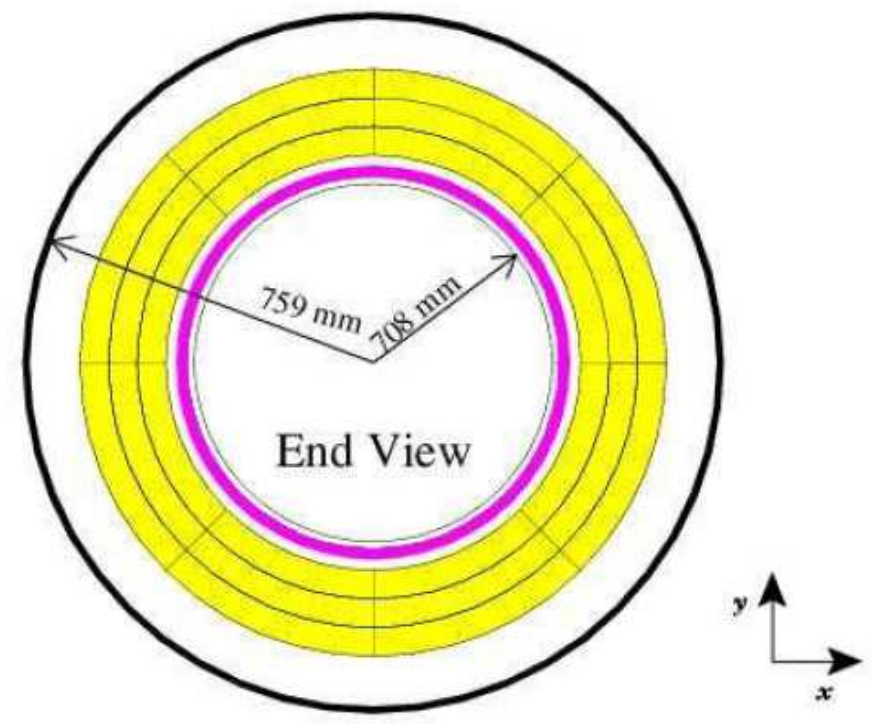

Figure 24. Schematic r-phi view of the central preshower detector.

particle (MIP) and shower. Sandwiched between the MIP and the shower regions is a $2 \mathrm{X}_{o}$ thick lead absorber plate covered in stainless steel. The MIP region locates the $\eta, \phi$, and $\mathrm{z}$ position of the trajectory of the charged particle while the shower layer measures the energy of the particle. A photon will not deposit energy in the MIP region but it may interact with the lead absorber depositing energy in the shower region. The two MIP layers have $\mathrm{u}$ and $\mathrm{v}$ stereo fibers covering $\Delta \phi=22.5^{\circ}$. The shower layers have a similar configuration. The detector is broken up into sixteen $22.5^{\circ}$ wedges, as shown in Figure 27. One $22.5^{\circ}$ wedge contains $1 u$ MIP layer, an absorber, and $1 \mathrm{v}$ shower layer. The next $22.5^{\circ}$ wedge contains $1 \mathrm{v}$ MIP layer, an absorber, and $1 \mathrm{u}$ shower layer. There are 7442 channels on each of the two forward preshower detectors. 


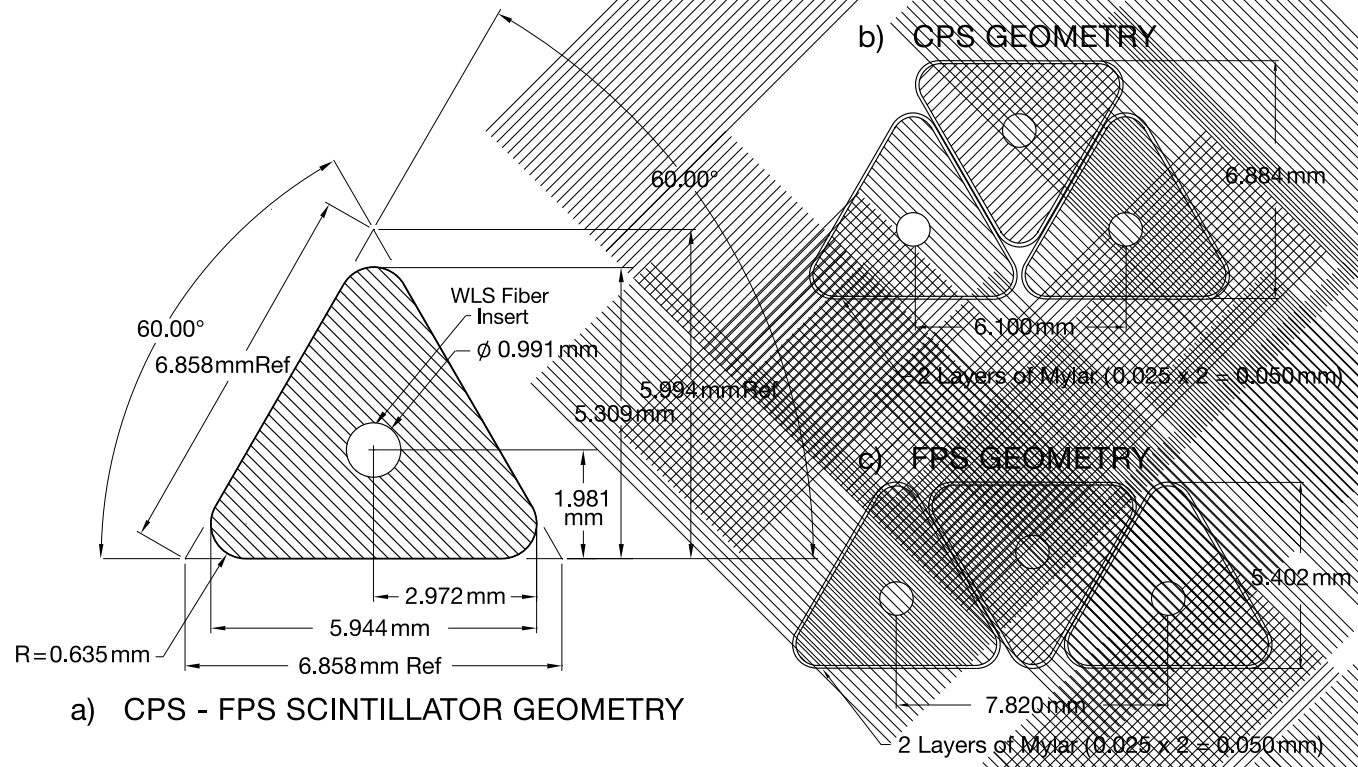

Figure 25. The cross section of the central and forward preshower scintillating triangular strips.

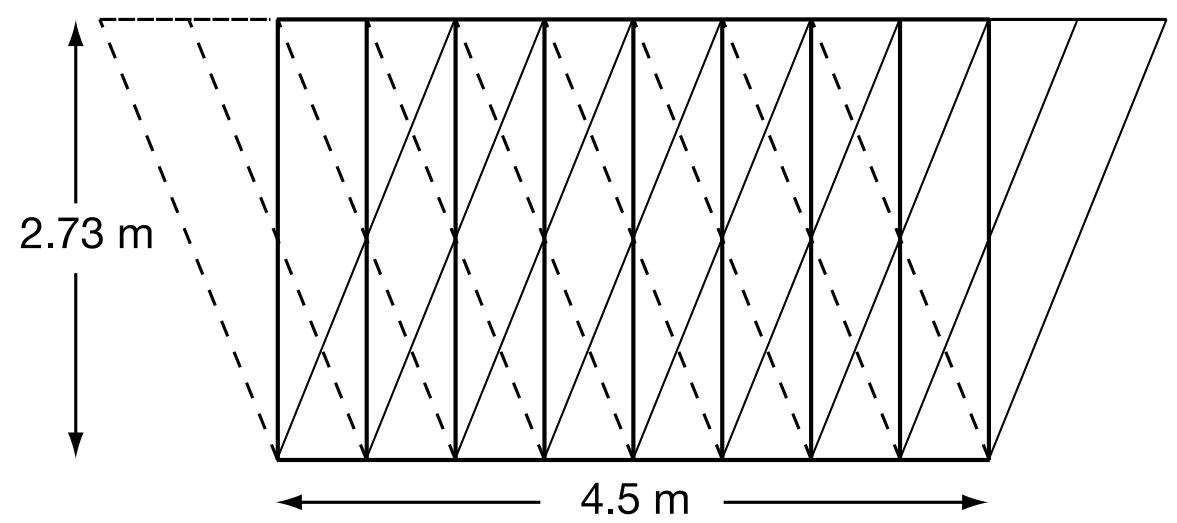

Figure 26. The layout of three layers of scintillating strips - one axial and two at stereo angles. 


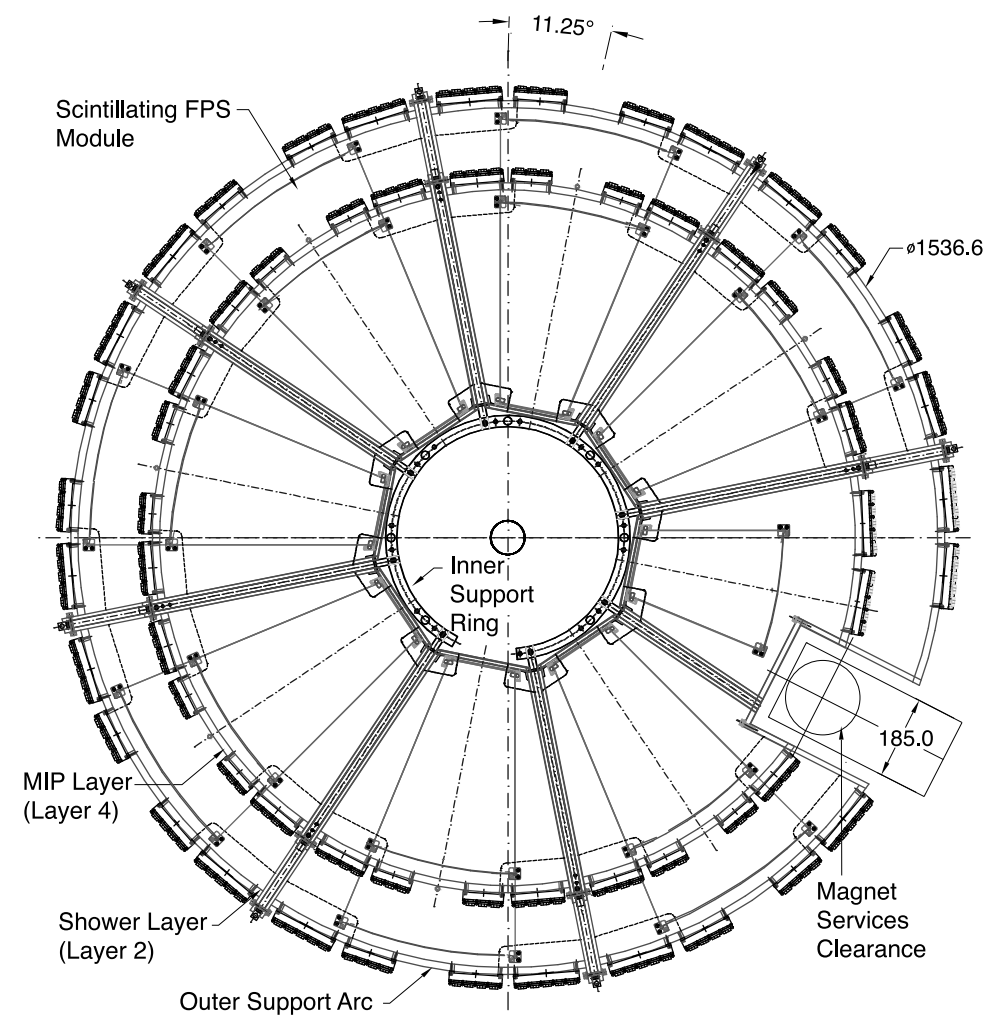

Figure 27. The forward preshower detectors attached to each of the calorimeter end caps. 


\subsubsection{The Calorimeters}

A calorimeter is a detector which intercepts the primary particle, and is of sufficient thickness to cause it to interact and deposit all its energy inside the detector volume in a subsequent cascade or "shower" of increasingly lower-energy particles (28). The main purpose of the calorimeter is to measure the energy and position of electrons, photons, and jets from $p \bar{p}$ collisions. The electrons/photons will shower in the calorimeter into lower energy electrons and photons with a transverse width of about $1-2 \mathrm{~cm}$ (Moliere radius). Hadrons interact with the nucleus of the absorber material creating a cascade of secondary particles. Half of the incident hadron energy is carried by low $p_{T}$ particles with the remainder of the energy carried by a few fast particles continuing the cascade. The transverse width of a hadron shower is typically 10 $\mathrm{cm}$.

The calorimeter (Figure 28) covers the pseudo-rapidity range of $|\eta|<4.0$. The calorimeter is broken up into three different detectors, the central calorimeter (CC) and two end calorimeters (EC). Each detector is housed within its own cryostat and maintained at $90^{\circ} \mathrm{K}$. The CC covers $|\eta|<1$.1. Each calorimeter is broken up into two sections: EM and hadronic. The hadronic section is further broken down into degrees of coarseness of the absorber plates. The central calorimeter has a fine hadronic region where the absorber plates (depleted uranium and $2 \%$ niobium) are $6 \mathrm{~mm}$ thick and a course hadronic region where the absorber plates (Copper) are 46.5 mm thick. The end calorimeters have an EM absorber section and three hadronic absorber regions: inner, middle, and outer. 


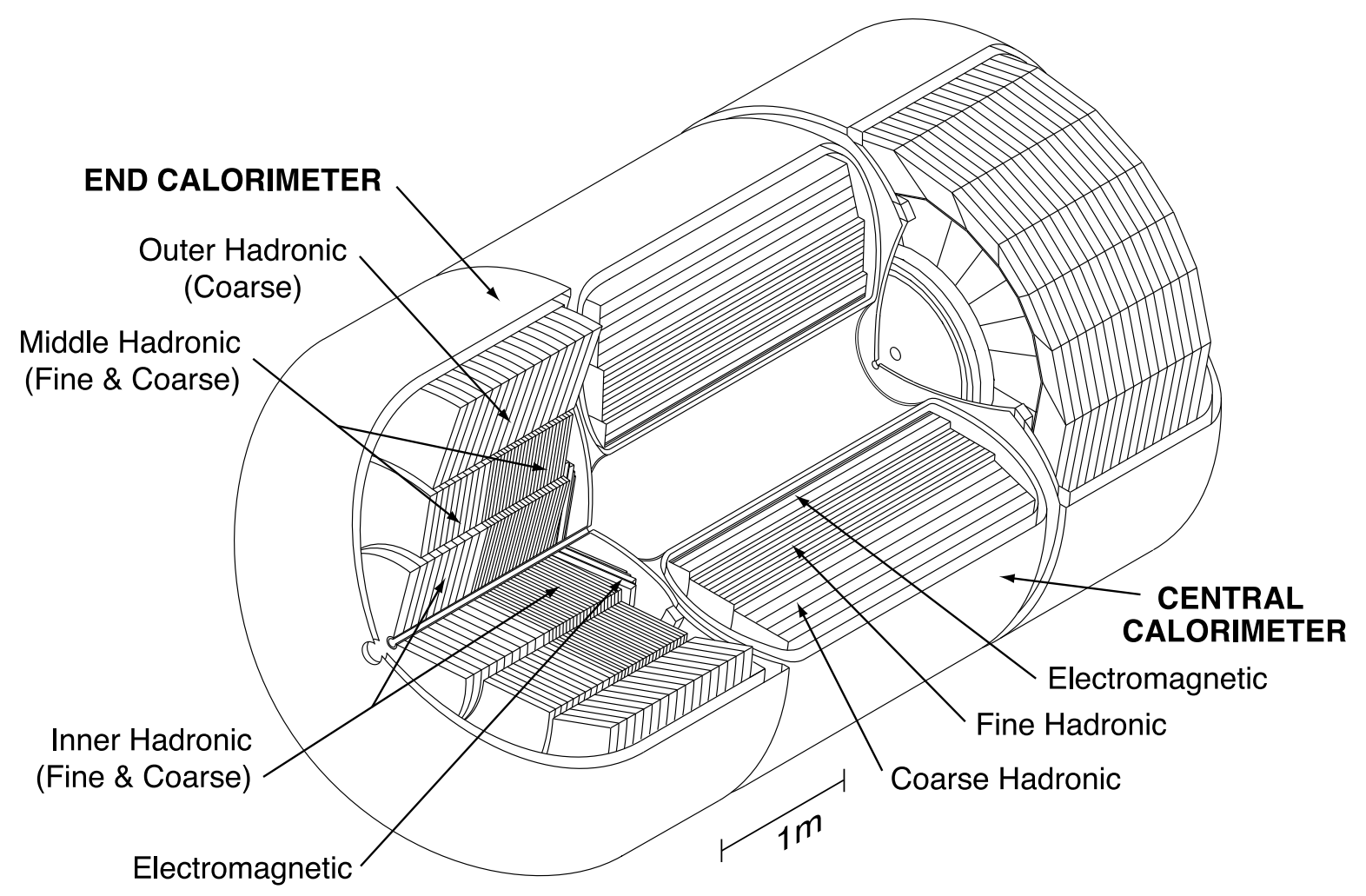

Figure 28. A view of the central and end cap calorimeters.

A typical unit cell is depicted in Figure 29. The unit cell contains an absorber plate, liquid argon gap, a signal board, followed by a liquid argon gap. The absorber plates are $3-46.5$ mm thick and held at zero voltage. The signal board is made of two copper-clad G10 sheets laminated together with a resistive epoxy coating covering the two surfaces that are exposed to the liquid argon. The two epoxy covered surfaces are held at around $2 \mathrm{kV}$. One G10 sheet has the inner copper clad coating removed, while the other G10 sheet has its inner copper clad coating milled for segmented readout. Liquid argon fills the $2.3 \mathrm{~mm}$ gap between the absorber 
and signal boards. Charged particles passing through the liquid argon form ions causing current to flow across the potential difference. A group of unit cells along the same trajectory form a layer. All layers along the same trajectory form a calorimeter readout tower of $0.1 \times 0.1$ in $\eta \times \phi($ Figure 30 and Figure 31).

3.2.5 shows the parameters for the CC. The EM section of the CC has four readout layers. The first two readout layers are made out of two unit cells each. The third readout layer has seven unit cells while the fourth layer has ten unit cells. The fine hadronic section for the CC has three readout layers with twenty, sixteen, and fourteen unit cells, respectively. The coarse hadronic section has one readout layer consisting of nine unit cells. 3.2.5 shows the detectors parameters for the EC.

\begin{tabular}{|lccc|}
\hline & $\mathrm{EM}$ & $\mathrm{FH}$ & $\mathrm{CH}$ \\
\hline Number of modules & 32 & 16 & 16 \\
Absorber $^{a}$ & $\mathrm{U}$ & $\mathrm{U}-\mathrm{Nb}$ & $\mathrm{Cu}$ \\
Absorber thickness $(\mathrm{mm})$ & 3 & 6 & 46.5 \\
Argon Gap $(\mathrm{mm})$ & 2.3 & 2.3 & 2.3 \\
Number of readout layers & 4 & 3 & 1 \\
Cells per readout layer & $2,2,7,10$ & $20,16,14$ & 9 \\
Total radiation lengths $\left(X_{o}\right)$ & 20.5 & 96.0 & 32.9 \\
Total nuclear absorption lengths $(\lambda)$ & 0.76 & 3.2 & 3.2 \\
Total readout cells & 10368 & 3000 & 1224 \\
\hline
\end{tabular}

${ }^{a}$ Depleted uranium (U), depleted uranium with $2 \%$ niobium (U-Nb), or copper $(\mathrm{Cu})$.

TABLE V

CENTRAL CALORIMETER PARAMETERS 


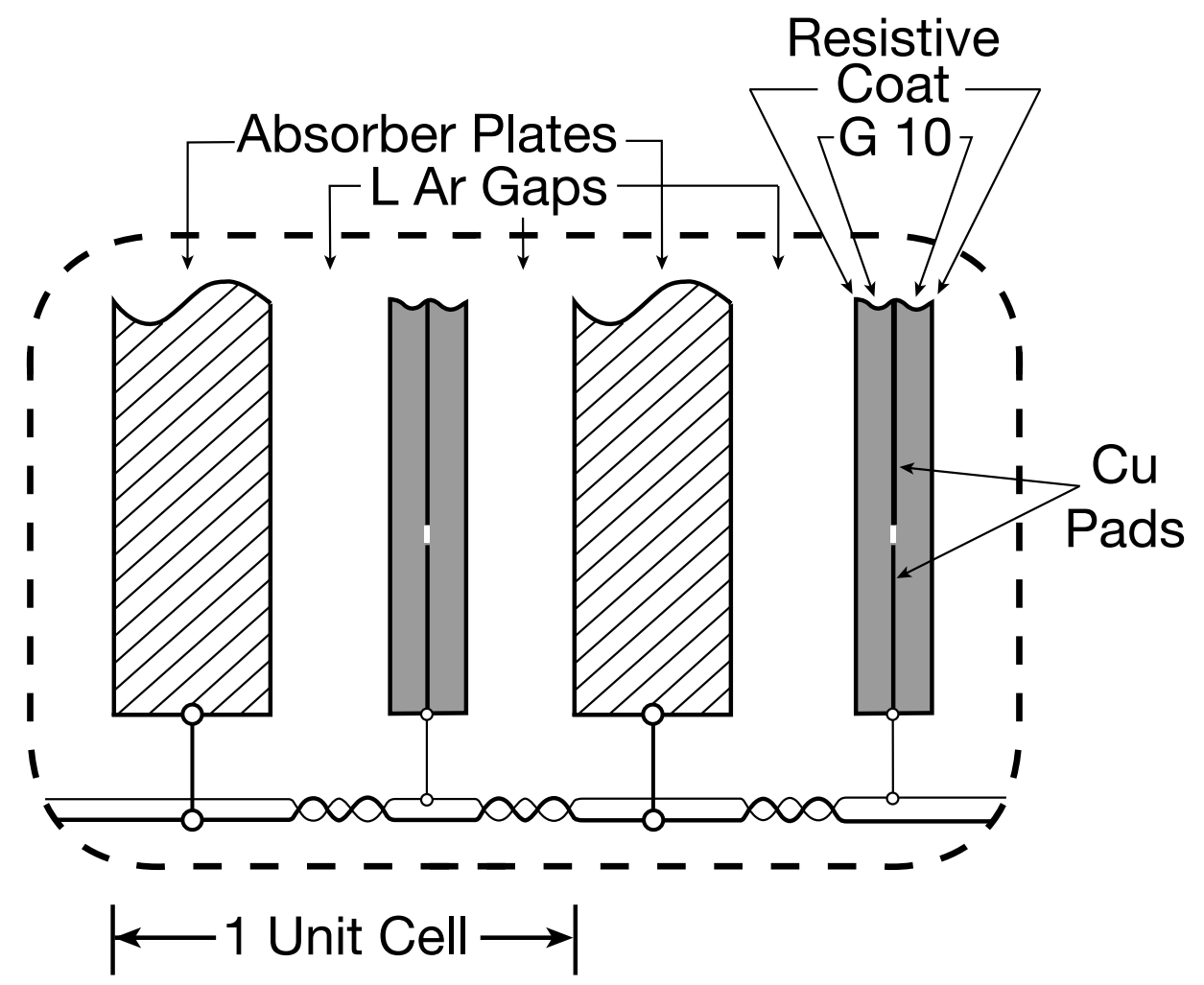

Figure 29. Schematic view of a unit cell in the calorimeter. 


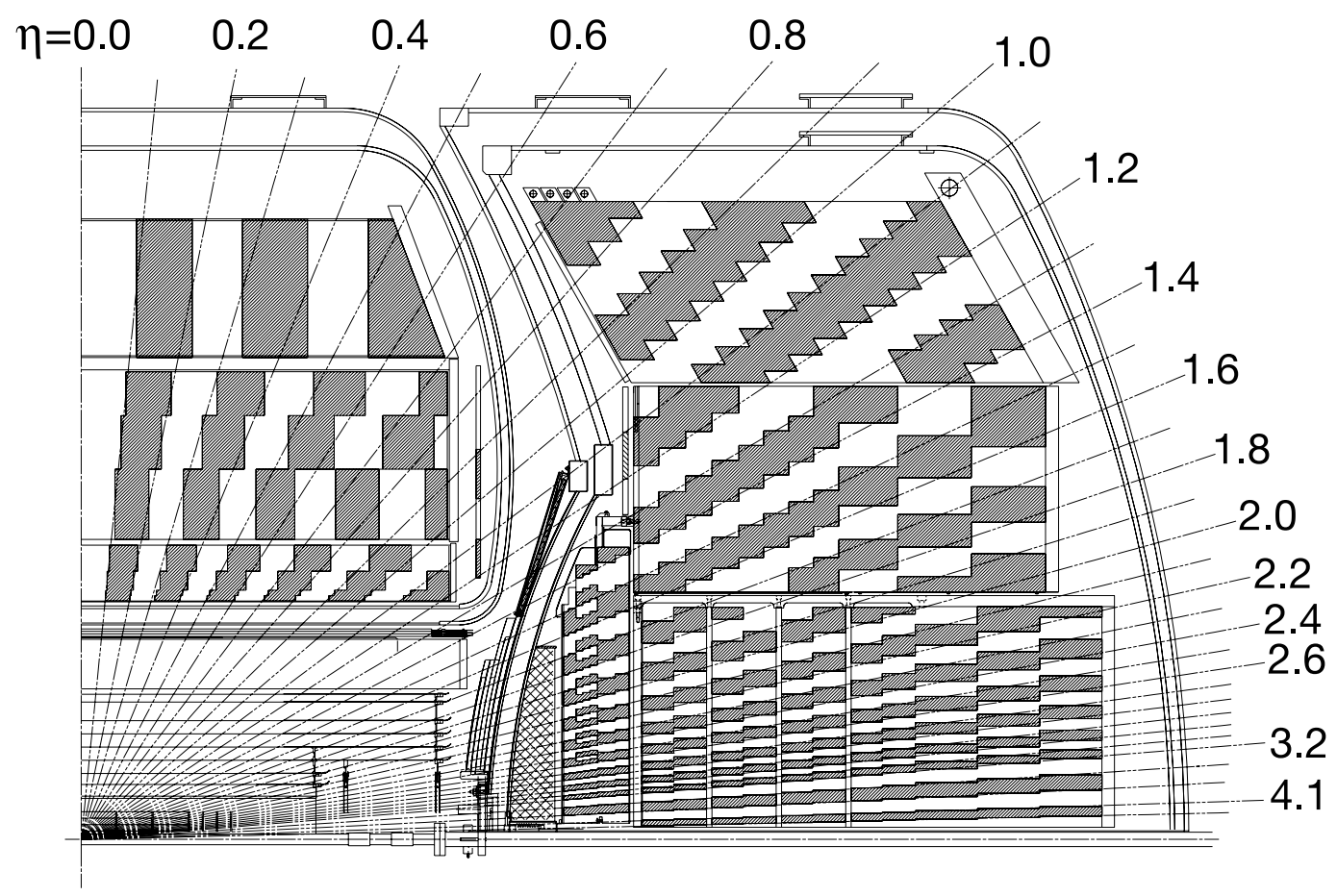

Figure 30. One quarter view of the calorimeter showing the segmented readout. 

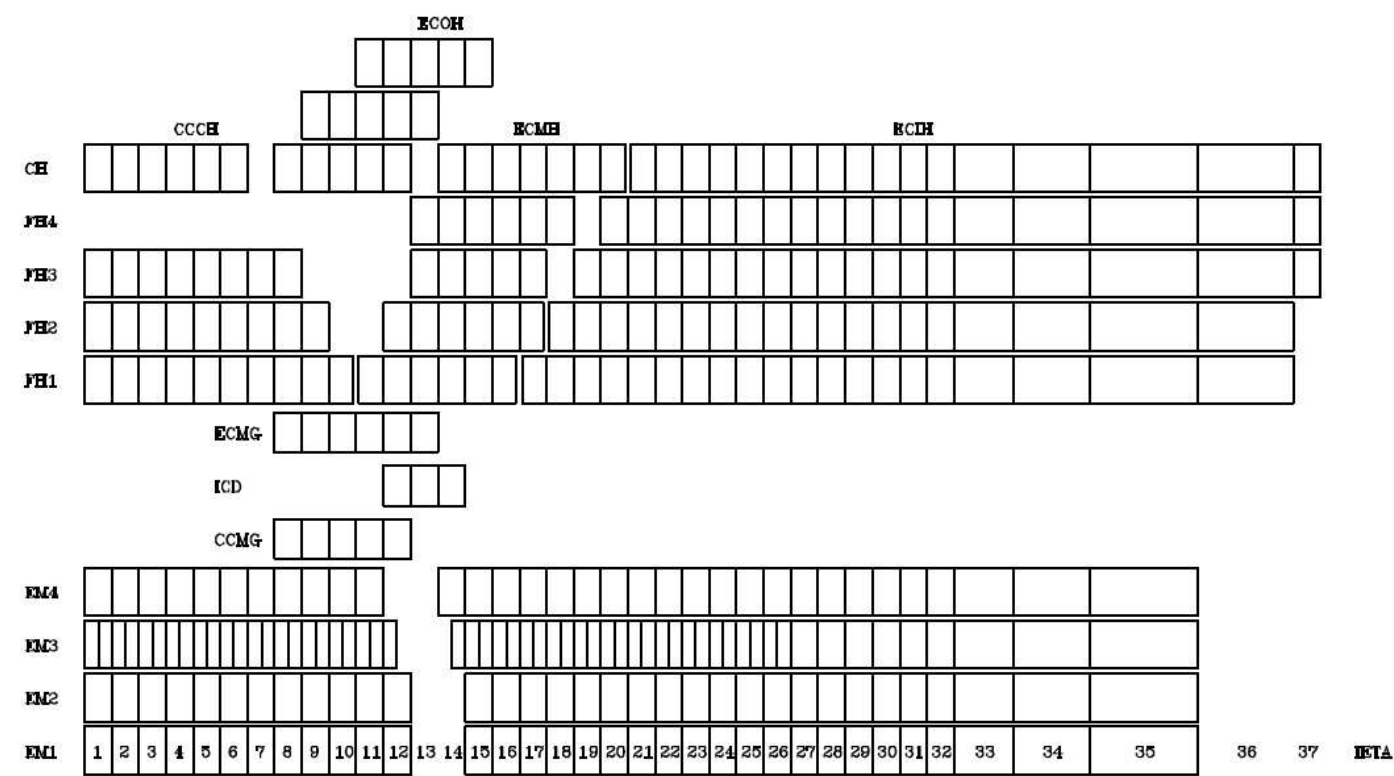

$$
\begin{array}{ccccccccccccccccccccccc}
\mid & 1 & 1 & 1 & 1 & \mid & 1 & 1 & 1 & 1 & \mid & 1 & 1 & 1 & 1 & \mid & 1 & 1 & 1 & 1 & \mid & 1 & 1 \\
0 & & & & 10 & & & & & & & & & & & 10 & & & & & & &
\end{array}
$$

Figure 31. The calorimeter readout towers in $\eta$ versus the layers distributed along the shower development. 


\begin{tabular}{|lcccccc|}
\hline & EM & IFH & ICH & MFH & MCH & OH \\
\hline Number of modules & 1 & 1 & 1 & 16 & 16 & 16 \\
Absorber $^{a}$ & $\mathrm{U}$ & $\mathrm{U}-\mathrm{Nb}$ & $\mathrm{SS}$ & $\mathrm{U}-\mathrm{Nb}$ & $\mathrm{SS}$ & $\mathrm{SS}$ \\
Absorber thickness $(\mathrm{mm})$ & 4 & 6 & 46.5 & 6 & 46.5 & 46.5 \\
Argon Gap $(\mathrm{mm})$ & 2.3 & 2.1 & 2.1 & 2.2 & 2.2 & 2.2 \\
Number of readout layers & 4 & 4 & 1 & 4 & 1 & 3 \\
Cells per readout & $2,2,6,8$ & 16 & 14 & 15 & 12 & 8 \\
Total radiation lengths $\left(\mathrm{X}_{o}\right)$ & 20.5 & 121.8 & 32.8 & 115.5 & 37.9 & 65.1 \\
Total nuclear absorption lengths $(\lambda)$ & 0.97 & 4.9 & 3.6 & 4.0 & 4.1 & 7.0 \\
Total readout cells & 7488 & 4288 & 928 & 1472 & $384+64$ & +896 \\
\hline
\end{tabular}

${ }^{a}$ Depleted uranium (U), depleted uranium with $2 \%$ niobium (U-Nb), or stainless steel (SS).

TABLE VI

END CALORIMETER PARAMETERS 
The EM detector for the central calorimeter is made up of thirty-two modules along $\phi$. The EM module boundaries, called " $\phi$ cracks", are uninstrumented regions. Electrons and photons passing through the $\phi$ cracks only deposit a fraction of their energy amongst the two adjacent $\phi$ modules. The fine hadronic section is broken up into sixteen modules along $\phi$ and centered on the EM module boundaries.

The four EM layers at $\eta=0$ have radiation lengths $\left(\mathrm{X}_{0}\right)$ of $1.4,2.6,6.8$, and 9.8 , respectively, totaling $20 \mathrm{X}_{o}$ and 0.76 interaction lengths $\left(\lambda_{I}\right)$. The hadronic layers at $\eta=0$ have a total of $129 \mathrm{X}_{o}$ and $6.4 \lambda_{I}$ of material. The $\mathrm{D} \varnothing$ calorimeter was designed to separate EM and hadronic showers by using a high:

$$
\frac{\lambda_{I}\left(\mathrm{~g} / \mathrm{cm}^{2}\right)}{X_{o}\left(\mathrm{~g} / \mathrm{cm}^{2}\right)} \sim \frac{35 A^{\frac{1}{3}} Z^{2}}{180 A} \sim 0.12 Z^{\frac{4}{3}}
$$

absorber material (depleted uranium).

The three calorimeter detectors have under-instrumented regions called the inter cryostat regions (ICR) caused by the physical boundaries of the cryostats. To improve the energy measurement, two additional types of detectors are added to the calorimeters. The first is a ring in $\phi$ of massless gap detectors covering $0.6<|\eta|<1.3$. The massless gap detectors are located inside the cryostats but outside of the absorber plate region. The massless gap detectors are similar to the unit cell detectors but they do not have absorber plates. The second ICR detector is a ring in $\phi$ of scintillator tiles covering $1.1<|\eta|<1.5$. The tiles are arranged to give segmented readout comparable to match a unit cell.

The readout of the calorimeter layers is characterized by a pre-amplifier, a Level-1 trigger pick-off, signal shaping, storage circuits, and analog to digital converter (ADC). The calorimeter 


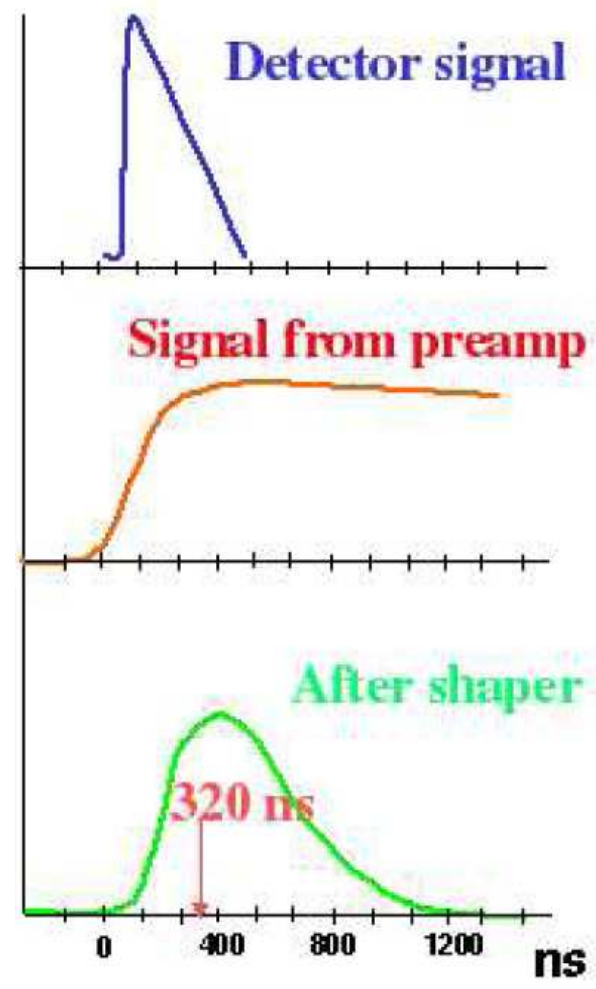

Figure 32. The shape of the signals detected and processed through the electronics in the calorimeter.

electronics samples the shaped signal every $132 \mathrm{~ns}$. The electron drift velocity across the 2.3 $\mathrm{mm}$ gap at $2 \mathrm{kV}$ is approximately $450 \mathrm{~ns}$. The pre-amplifier has a rise time of $450 \mathrm{~ns}$ and a decay time of $15 \mu \mathrm{s}$. The pre-amplifier signal does not fall quickly enough for the voltage to be at zero for the next collision. This signal tail produces a baseline DC offset for the next interaction, Figure 32. The pre-amplifier signal is sent to the Base Line Subtracter (BLS) board which can hold the signal for $4 \mu s$, waiting for a Level-1 trigger decision (Figure 33). The BLS boards provide base line subtraction of the pre-amplifier signal, and fast shaped analog sums of 


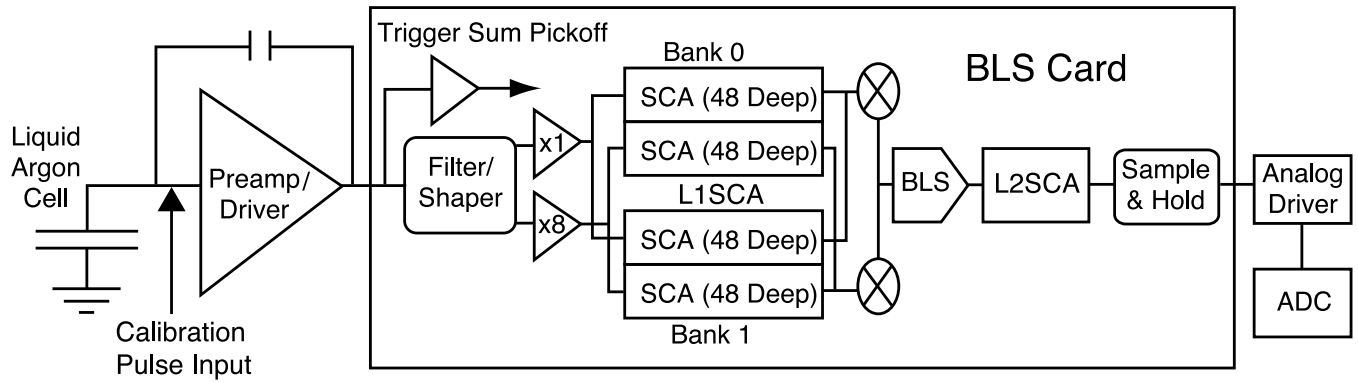

Figure 33. The electronic readout of the unit cell to the analog digital converter.

the signal for Level-1 and Level-2 trigger decisions. The fast pick-off analog sums arrange EM and EM+hadronic energies in towers of $0.2 \times 0.2$ in $\Delta \eta \times \Delta \phi$. Only $2 / 3$ of the pre-amplifier signal or $260 \mathrm{~ns}$ is used for the signal shaper circuit. The shaped signal has a peak at $320 \mathrm{~ns}$ with a $1.2 \mu \mathrm{s}$ decay. After a Level-1 accept ${ }^{1}$ the signal is transfered into a forty event deep buffer before transfer to Level-3.

${ }^{1}$ Described in Chapter 4. 


\subsubsection{The Muon System}

The muon spectrometer $(29 ; 30)$ is designed to detect muons, which are minimum ionizing particles at transverse momenta greater than $1 \mathrm{MeV}$. Therefore, the muons penetrate through all of the detectors and only leave small energy deposits in the tracking system, calorimeter, and muon spectrometer. The muon spectrometer consists of proportional drift tubes, mini drift tubes, scintillator counters, and a $1.8 \mathrm{~T}$ iron toroid magnet. The muon-detector system has three layers: A, B, and C. The A layer is in between the calorimeter and the toroid. The B and C layers are located outside of the toroid (Figure 11). The scintillator counters mounted inside (layer A) and outside (layers B and C).

The proportional drift tubes (PDT) detect the ionization charge when muons interact with the gas inside the tubes. The $10.1 \mathrm{~cm}$ wide tubes are rectangular in shape and have a $50 \mu \mathrm{m}$ gold plated tungsten wire parallel to the toroid magnetic field (Figure 34). An anode wire is located at the center of each tube and held at $4.7 \mathrm{kV}$. Along the inner walls of the tube are vernier cathode pads which are placed above and below the wire and held at $2.3 \mathrm{kV}$. The gas $\left(84 \%, 8 \% \mathrm{CF}_{4}, 8 \% \mathrm{CH}_{4}\right)$ is allowed to flow through the tubes. The maximum electron drift time is $500 \mu \mathrm{s}$. The hit resolution of the PDTs is $5 \mathrm{~mm}$. The A layer consists of four layers of drift tubes, while the B and C layers consists of three layers of drift tubes.

The mini drift tubes (MDT) are similar to the proportional drift tubes. The MDTs are

smaller, $9.4 \times 9.4 \mathrm{~mm}^{2}$, do not have vernier cathode pads, and contain a slightly different mixture of gas $\left(90 \% \mathrm{CF}_{4}, 10 \% \mathrm{CH}_{4}\right)$. The MDTs are in the forward muon system covering 1.1 $<|\eta|<2.2$. The tubes contain a $50 \mu \mathrm{m}$ gold plated tungsten wire held at ground (Figure 35). 


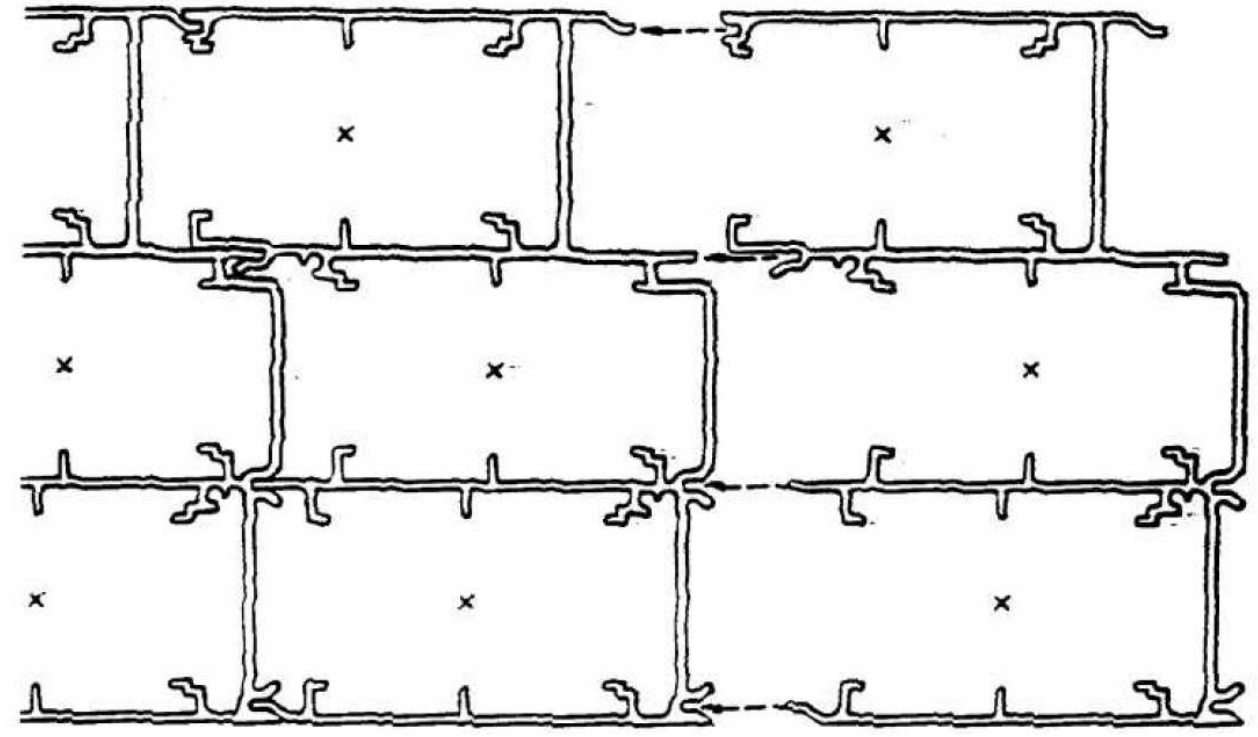

Figure 34. Cross section through proportional drift chambers.

A negative voltage $3.2 \mathrm{kV}$ is applied to the cathode. The electron drift time is $132 \mathrm{~ns}$, and the hit resolution of the MDTs is $0.7 \mathrm{~mm}$. 


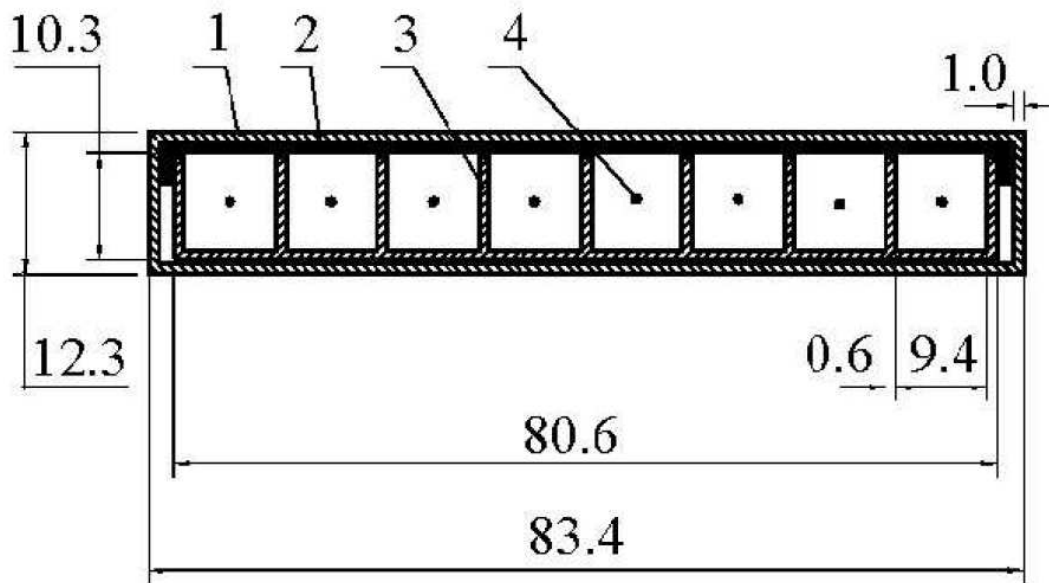

Figure 35. A cross section of the mini-drift tubes. 
The scintillation counters are used for position measurements and a precise measurement of the muon arrival times. Cosmic ray muons are rejected using the arrival times of the muons. The scintillators are a half inch thick and are made of Bicron 404A scintillator with a wave-shifting fiber placed in a milled out groove on the scintillator. Photomultiplier tubes are attached to the ends of the wave-shifter fibers for readout. The fast response and readout of the scintillation counters are used for Level-1 trigger decisions. The scintillators are segmented into regions of 4.5 $5^{\circ}$ matching the CFT segmentation. The CFT tracks and muon scintillator hits are matched for Level-1 trigger decisions.

The toroid magnet is broken down into six separate sections forming a cube around the calorimeter. The toroid is a square annulus of $109 \mathrm{~cm}$ thickness. The inner surface is $318 \mathrm{~cm}$ from the beam line. The iron magnet is wound using coils of ten turns each. A current of 1500 A is applied to the coils and the resulting magnetic field is $1.8 \mathrm{~T}$. Figure 36 shows a simulated deflection of a muon through the magnetic field produced by the toroid. 


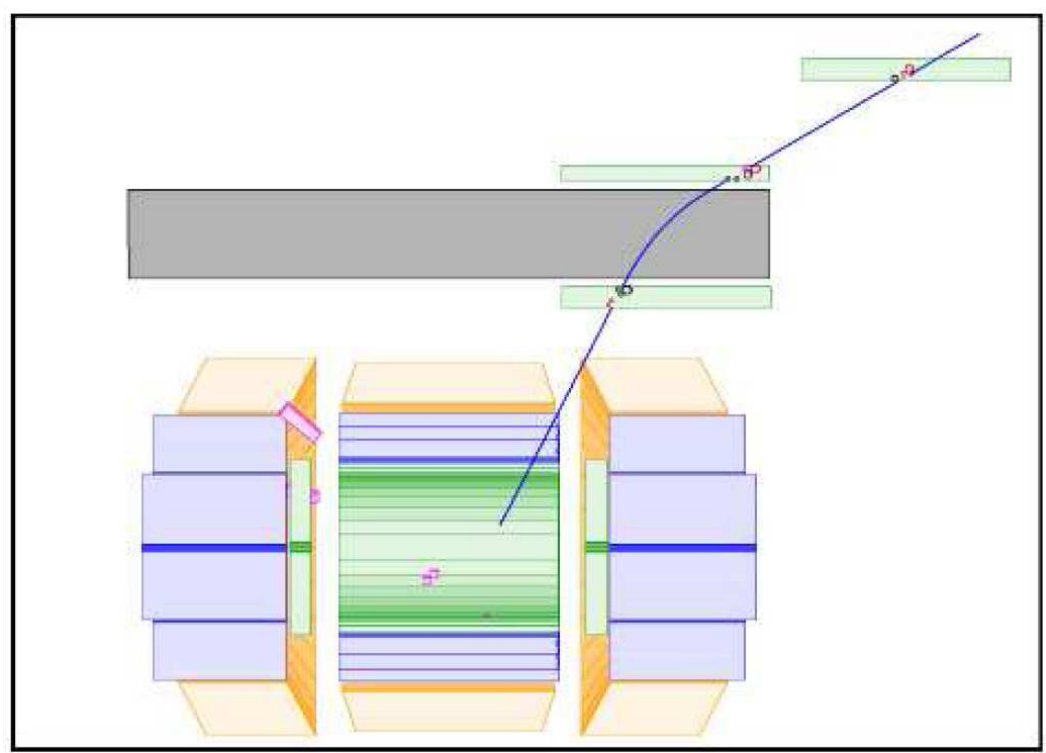

Figure 36. Simulated muon trajectory through the D $\varnothing$ detector. 


\section{CHAPTER 4}

\section{THE DØ TRIGGER AND DATA ACQUISITION SYSTEMS}

\section{1 $\quad$ Trigger overview}

The Tevatron collides 36 bunches of protons with 36 bunches of anti-protons at a collision rate of $1.7 \mathrm{MHz}$. The average amount of data collected by the $\mathrm{D} \varnothing$ detector per event is 250 $\mathrm{kB}$. Collecting data from every collision would require storing approximately $425 \mathrm{~GB}$ of data per second which is not feasible. In addition, interesting high energetic $p \bar{p}$ collisions have a low probability to occur. The selection of such collisions from the elastic and soft inelastic events is done via a three level trigger system (31). The Level-1 (L1) trigger system (32) uses a hardware trigger to reduce the rate by a factor of 1000 . The Level-2 (L2) trigger system $(33 ; 34)$ reduces the rate by a factor of 2-5, and the Level-3 (L3) trigger system (35) reduces the rate by a factor of 10. The data are stored on local online disks at an average rate of 50-100 $\mathrm{Hz}$ for further transmission to permanent tape storage. The amount of data stored per second is about 12.5 MB.

The data acquisition is controlled by the COOR (36) software package (Figure 37). COOR receives the trigger list, communicates with the trigger framework, relays the trigger decision parameters, and controls the data flow. The trigger list specifies the requirements which can fire an event at each trigger level. The trigger list contains about 450 L3 trigger conditions 
that correspond to specific L1 and L2 trigger bits. The L1 and L2 systems have a one-to-one correspondence of the 128 possible triggers bits.

Figure 37 shows the flow of data from the detector to storage on tape. With a beam crossing rate of $1.7 \mathrm{MHz}$, the detector information is passed to the L1 trigger. Once a L1 condition is satisfied, the trigger framework (TFW) sends a L1 Accept to the detector sub-systems, and the detector information is sent to the L2 system. Once the L2 trigger generates a decision, it passes its decision to the TFW. The TFW then communicates to the detector sub-systems to send the data to L3. The data acquisition system (DAQ) is responsible for the coordination of the L3 farm nodes and the online run control. Once the data is inside a L3 farm node, the event is processed through a nearly complete event reconstruction and the final trigger decision is made.

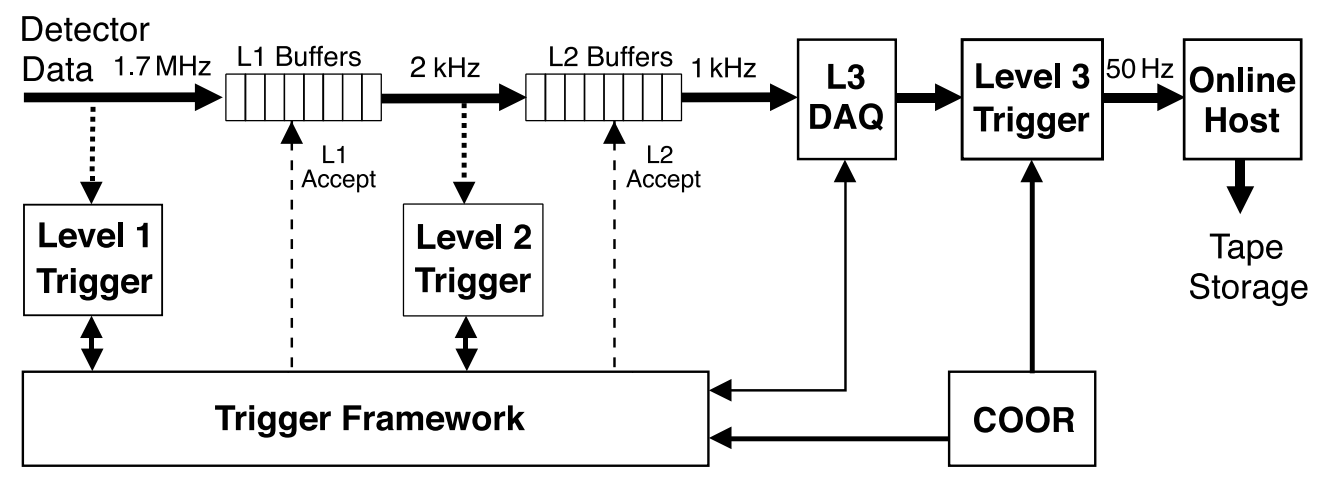

Figure 37. The flow of the data from the $D \varnothing$ detector to storage onto magnetic tapes. 


\subsection{The Level-1 Trigger}

The L1 trigger (Figure 38) generates decisions based on requirements from the tracking, calorimeter, and muon detectors. The L1 trigger decision, made by the TFW, is based on 128 individual trigger conditions OR-ed together on a framework of FPGAs. The digitized readout systems have enough memory to hold 32 bunch crossings.

\subsubsection{The Trigger Framework}

The TFW makes the decision whether a particular event is to be accepted for further examination by using the inputs of the L1 trigger devices. The TFW uses the logical "OR" of up to 128 specific triggers conditions defined by the trigger list to determine if a given crossing holds a valid trigger. The TFW also manages the rates of triggers by applying prescale factors to keep their rates within acceptable limits. Different trigger lists and prescale settings are passed by COOR using the trigger control computer (TCC). The TFW provides a large number of scalars to monitor trigger rates and dead times.

\subsubsection{The Level-1 Calorimeter Trigger}

The Level-1 calorimeter (L1Cal) trigger counts the number of times EM and EM+HAD calorimeter towers pass a set of $\mathrm{E}_{T}$ thresholds. L1Cal forms $\Delta \eta \times \Delta \phi=0.2 \times 0.2$ trigger towers. There are forty trigger towers in $\eta$ covering $|\eta|<4.0$. Each slice in $\eta$ has thirty-two trigger towers covering the full $2 \pi$ of the azimuth $(\phi)$. The counts are passed to the TFW and the trigger towers are passed to the L2 calorimeter system on a L1 Accept. 


\subsubsection{The Level-1 Central Track Trigger}

The Level-1 Central Track Trigger (L1CTT) reconstructs trajectories of charged particles using the axial hits from the CFT and the PS detectors. The CFT and CPS axial system provide triggers for charged particles using predefined track equations and matching tracks to PS clusters. The CFT and CPS axial track list is passed to L1Muon, and to the L2 tracking preprocessors on L1 Accept. L1CTT conditions can be specified by the number of tracks above a $p_{T}$ threshold, with or without a PS cluster match, and track isolation.

\subsubsection{The Level-1 Muon Trigger}

The Level-1 Muon trigger (L1Muon) uses hits from the muon wire chambers, muon scintillator counters, and tracks from L1CTT to form patterns consistent with muons. L1Muon forms trigger objects based on L1CTT tracks and muon scintillator hits. L1Muon also forms trigger objects based on matching layers of track stub (group of interlayer hits) wire hits which have been confirmed with muon scintillators. L1Muon conditions can be specified by the number of muons above a $p_{T}$ threshold, geographical region, and track quality. 


\subsection{The Level-2 Trigger}

The L2 trigger system was designed to operate within $\mathrm{a} \approx 100 \mu$ s time window and to reduce the L1 rate by a factor of 10 . During physics data taking, the L2 trigger typically receives events at a rate of $1.5 \mathrm{kHz}$ and has a rejection factor from two to five.

The L2 trigger system consists of six separate elements as shown in Figure 38: five individual preprocessors and one trigger decision processor, L2 Global (L2GBL). Each preprocessor generates trigger objects: muons, electrons, photons, jets, tracks, and preshower clusters, and L2GBL combines them to form the final L2 trigger decision. As an example, a L2 trigger could require one jet and one muon above a $p_{T}$ threshold. L2GBL can have up to 128 separate trigger decisions to process before accepting or rejecting an event. If the event passes at least one trigger, then this event is passed along to the L3 trigger system for further review.

Detailed information about the data flow inside the L2 trigger system can be found in Appendix A. Detailed information about some of the hardware components of the L2 trigger system can be found in Appendix B.

\subsubsection{Level-2 Global Processor}

L2GBL receives trigger objects from the L2 preprocessors (L2 Calorimeter, L2 CTT, L2 Muon, and L2 PS). L2GBL uses the trigger list and the L1 trigger decision mask to decide which script to run on the objects. Each script is defined by at least one or more filters and a minimum number of objects required to pass each filter. As an example, a script could have an EM object filter with a minimum of one object. This script will pass the event if there is 


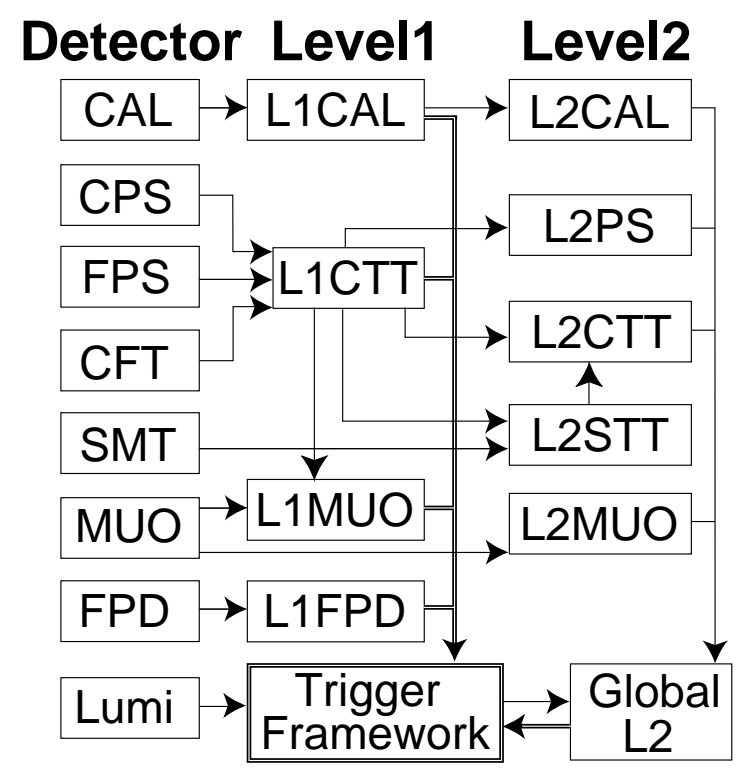

Figure 38. The flow of data from the L1 inputs to the Level-2 trigger.

at least one EM object in the event that satisfies the conditions of the EM filter. Detailed information about EM filters and tools can be found in Appendix D.

\subsubsection{Level-2 Calorimeter Preprocessor}

The L2 calorimeter preprocessor (L2Cal) runs EM and jet algorithms. The two algorithms receive a list of $1280 \mathrm{EM}$ and $1280 \mathrm{EM}+\mathrm{HAD}$ trigger towers from $\mathrm{L} 1 \mathrm{Cal}$ to form the $\mathrm{EM}$ and Jet objects. The EM algorithm forms electron and photon objects using EM trigger towers. A cluster is formed by a seed trigger tower with $E_{T}>1 \mathrm{GeV}$ and its largest $E_{T}$ neighboring EM tower. EM fraction and isolation fraction are calculated for each EM object. The jet algorithm forms jet objects by clustering $5 \times 5 \mathrm{EM}+\mathrm{HAD}$ trigger towers centered around a seed tower with $E_{T}$ greater than $2 \mathrm{GeV}$. The EM and jet objects are independently sorted in descending 
order of clustered $E_{T}$ and sent to L2GBL. More detailed information about the EM and jet algorithms can be found in Appendix C.

\subsubsection{Level-2 Silicon Track Trigger Preprocessors}

The L2 silicon track trigger preprocessor (L2STT) uses L1CTT tracks and SMT hits to improve the momentum resolution of the tracks and to calculate track impact parameters ${ }^{1}$. The impact parameter calculation from L2STT is used to tag the decays of long-lived particles such as B hadrons. The L2STT algorithm fits track parameters by projecting L1CTT tracks into the SMT detector. The fitted tracks are sent to L2CTT.

\subsubsection{Level-2 Central Track Trigger Preprocessor}

The L2 Central Track Trigger preprocessor (L2CTT) sends tracks to L2GBL to match tracks to physics objects. L1CTT tracks are combined and sorted in descending order of $p_{T}$. The azimuthal angle, $\phi_{o}$, with respect to the beam axis is determined, as well as the azimuthal angle of the track projected to the third EM layer of the calorimeter $\phi_{e m 3}$. Several isolation criteria are calculated to enhance the trigger capabilities. Similarly in L2STT, tracks are combined and sorted, and azimuthal angles and isolation criteria are evaluated. The L2CTT processor sends three lists of tracks to L2GBL: a $p_{T}$ sorted list of L1CTT tracks, a $p_{T}$ sorted list of L2STT tracks, and an impact parameter sorted list of L2STT tracks.

\footnotetext{
${ }^{1}$ The perpendicular distance from the original center of a set of scattering particles to the original line of motion of a particle being scattered.
} 


\subsubsection{Level-2 Preshower Preprocessor}

The L2 Preshower preprocessor (L2PS) is used to improve the electron detection efficiency and photon separation. All three layers of the PS detectors are used to form clusters in $\eta$ and $\phi$. L2PS also reports L1CTT tracks that match with PS clusters.

\subsubsection{Level-2 Muon Preprocessor}

The L2 Muon preprocessor (L2Muon) combines track segments among small regions and layers of the detectors in preprocessing units called Second Level Input Computers (SLICs). The sub layers are combined into integrated muon candidates in the preprocessor. The muon candidates are sorted in descending order of $p_{T}$ and are sent to L2GBL. The quality and timing information per muon candidate are also passed to L2GBL.

\subsection{Level-3 Trigger and Data Acquisition}

The L3 trigger system is a software based trigger running a fast version of the event reconstruction algorithms in order to reduce the event rate to 50-100 Hz. The trigger software runs on a farm of more than 100 commodity, dual $1 \mathrm{GHz}$ processors, rack-mounted PCs (37)

running Linux. Each detector system passes the readout information through commodity VME (38) single board computers (SBC) (38) via $100 \mathrm{Mb} / \mathrm{s}$ ethernet links through a Cisco (39) 6509 switch to the farm nodes. The L3 trigger decisions are based on complete physics objects as well as the relationships between the objects. 


\section{CHAPTER 5}

\section{OFFLINE EVENT RECONSTRUCTION AND OBJECT IDENTIFICATION}

This chapter describes how candidate electrons, jets, tracks, and vertices are reconstructed from raw detector data. A collection of complex software algorithms written in $\mathrm{C}++$ called DØreco (40) is used for the reconstruction process. DØreco unpacks the raw data, applies detector specific calibration constants, reconstructs tracks, generates a list of primary and secondary vertices, and identifies candidate objects like electrons, muons, and jets.

\subsection{Track Reconstruction}

Charged particles traversing a magnetic field leave traces along their paths when they interact with the SMT and CFT detectors. A typical event contains $10^{4}$ to $10^{6}$ hits in the central tracking system. Since charged particles can deposit energy among two adjacent silicon strips or two adjacent scintillating fibers, the hits are clustered together. The track reconstruction algorithm uses the hit clusters to find tracks. There are two track finding algorithms: Histogramming Track Finder (HTF) (41) and Alternative Algorithm (AA) (42).

The HTF method reconstructs tracks in two steps: (a) it uses a pattern recognition algorithm (histogramming) and (b) it does track fitting using a Kalman fitter (43). In the (r, $\phi)$ plane the charged particles travel in circular orbits and can be uniquely defined by three

parameters, $\rho, \mathrm{d}_{o}$, and $\phi$, where $\rho=\frac{q B}{p_{T}}, \rho$ is the curvature of the track, $\mathrm{d}_{o}$ is the distance of 
closest approach to the beam spot, and $\phi$ is the direction of the track at the position of closest approach to the beam spot. For every pair of hits in (x, y) space there is a corresponding point (bin) in the $2 \mathrm{D}(\rho, \phi)$ space which is histogrammed. All hits forming a track have multiple pair combinations. All pair combinations will have the same value (bin) in the $(\rho, \phi)$ histogram. The pattern recognition (track hypothesis) is made by taking a single hit in (x, y) and extrapolating it to be a line in the $(\rho, \phi)$ histogram. All hits from the same track will have separate lines which will all intersect at the same bin, the true $(\rho, \phi)$ of the track in question.

The track list (TrackL) is passed to a 2D Kalman filter which uses $\rho, \mathrm{d}_{o}$, and $\phi$ of each track, an expectation propagator, material effects (multiple scattering and energy loss), and the nonuniformity of the magnetic field to filter the TrackL. The remaining tracks pass through another histogramming algorithm which uses the hit $(\mathrm{r}, \mathrm{z})$ locations to form lines in $\left(\mathrm{z}_{o}, \mathrm{C}\right)$ space. $\mathrm{z}_{o}$ is the starting location of the track along the $\mathrm{Z}$ axis and $\mathrm{C}=\frac{d z}{d r}$. The lines which overlap in the $\left(\mathrm{z}_{o}, \mathrm{C}\right)$ histogram generate a reduced TrackL. The list is processed through an $\eta$ splitter, which only allows hits moving away from the interaction point to be associated with a track when the $\mathrm{z}$ component of the hits are increasing for $\eta>0$, and similarly when the $\mathrm{z}$ component of the hits are decreasing for $\eta<0$.

A 3D Kalman filter is then used to build the SMT tracks and continues including hits in the CFT detector until there are too many misses in a row or the algorithm comes to the end of the detector. Beginning with the partially reconstructed track, the 3D Kalman filter extends the track by an additional measurement or hit. The track parameters and the expectation propagator are used to make an expected hit measurement. A $\chi^{2}$ value is calculated and if 
the value is within the maximum allowed limit, the hit is accepted, the track parameters are recalculated for this track, and the algorithm repeats itself by searching for the next hit.

An additional HTF list is generated by starting from the CFT instead of the SMT using the same techniques. The two track lists are merged into one list and duplicate tracks are removed.

The AA uses three hits in the SMT layers for pattern recognition and then applies a track filter algorithm. For the pattern recognition, the first hit can come from any of the six layers in the silicon barrels or F disk. The second hit has to be within an azimuthal window, with respect to the beam spot, of less than 0.08 radians. The third hit has to be within a radius of curvature (when including the other two hits) $>30 \mathrm{~cm}$ (corresponding to a track with $p_{T} \geq$ $180 \mathrm{MeV}$ ), to have a track hypothesis within $2.5 \mathrm{~cm}$ of the axial impact point (at a distance of closest approach), and to have a $\chi^{2}$ value of the fit less than 16 . The fitting algorithm uses the track hypothesis, which gives expectation locations (search windows) for the hits at the next layers, and hits are added to the track if the $\chi^{2}$ value remains $<16$. The fitting algorithm continues until it has three consecutive misses in a row, or it reaches the end of the detector. The tracks are ordered by greatest number of hits, followed by the smallest number of misses, followed by the smallest $\chi^{2}$ value. Since the fitted tracks may share hits from other tracks, the AA requires that the number of hits shared to be less than $2 / 3$ of the total number of hits in the track.

The two track lists from the HTF and AA algorithms are combined into a single list, duplicates are removed, and the tracks are ordered in the same way as in the AA algorithm. 


\subsection{Primary and Secondary Vertex Reconstruction}

The primary vertices $(\mathrm{PVs})$ are the interaction points of $p \bar{p}$ collisions. Secondary vertices (SVs) are vertices displaced from the PV coming from the decay of long-lived particles such as $\mathrm{B}, \mathrm{K}_{s}$, or $\mathrm{D}$ hadrons. The $\mathrm{x}$ and $\mathrm{y}$ location of the PV fluctuates within $40 \mu m(1 \sigma)$ between events. The $\mathrm{z}$ location of the PV is roughly a Gaussian distribution with a spread $(\sigma)$ of $28 \mathrm{~cm}$.

The PVs are identified in two steps (44). First, the algorithm finds an approximate location of the beam spot and PV candidates. Second, the algorithm uses the beam spot information and tighter cuts on the tracks to identify the location of the PV. The beam spot is reconstructed by using tracks with a distance of closest approach significance $\left(\mathrm{dca} / \sigma_{d c a}\right)$ of less than 100 with respect to the detector center in $(r, \phi)$ coordinate space. All of the tracks are used to fit a location of the PV and the $\chi^{2}$ contribution of each track with respect to the vertex is computed. The track with the highest $\chi^{2}$ contribution is removed from the sample and the vertex is re-fitted with the remaining tracks. A new $\chi^{2}$ contribution for each track using the re-fitted vertex is computed. The process is repeated until the fitted vertex $\chi^{2}$ is less than ten. Once an approximate location of the beam spot is found, all the tracks which were not used in locating the approximate vertex location of the beam spot are used to find other vertex locations. After all of the vertices are found, the second step of the algorithm uses a new group of tracks (tighter cuts on the tracks) to fit the vertices based on their dca $/ \sigma_{d c a}<5$ with respect

to the $(r, \phi)$ position of the vertices found in the previous step. All tracks in the final fit of the PV must have $p_{T}>0.5 \mathrm{GeV}$, at least two SMT hits, and dca $/ \sigma_{d c a}<5$. The vertices are 
re-fitted and the track with the largest $\chi^{2}$ contribution is removed until the vertex $\chi^{2}$ value is less than ten.

The final selection of the hard collision PV from the soft inelastic vertices comes from a $\log _{10}$ $p_{T}$ track distribution of a Monte Carlo simulation of minimum bias events. The distribution is converted into a probability distribution (45). A probability value for each track in each vertex is extracted from the probability distribution. For each vertex, the track probabilities are multiplied together and weighted so that the final probability value does not depend on the number of tracks associated with the vertex. The vertex with the lowest probability is considered to be the hard collision PV. The uncertainty of the $\mathrm{x}$ and $\mathrm{y}$ positions of the hard collision PV is approximately $6 \mu \mathrm{m}$ each.

SVs are produced by using two tracks which do not point to the PV to construct a seed

vertex position. Other tracks are added and the vertex position is re-fitted. If the $\chi^{2}$ increases due to the addition of the new track, the new track is removed.

\subsection{Electromagnetic Object Reconstruction}

The EM object reconstruction algorithm (46) generates electron and photon objects from calorimeter towers. Since photons do not leave signals in the tracking system, a track matched to the energy deposit in the calorimeter provides a tool to distinguish electrons from photons.

EM object reconstruction begins with the formation of initial calorimeter clusters. This analysis uses the simple-cone tower clustering algorithm ("Scone Method"). The simple-cone algorithm clusters calorimeter towers based on precision readout data around seeds with $E_{T}>$ 
1.5 GeV in a cone of radius $\Delta R=\sqrt{\Delta \eta^{2}+\Delta \phi^{2}}<0.2$. All clusters satisfying the above criteria are tested for isolation:

$$
\text { Isolation }=\frac{E_{\text {total }}(\Delta R<0.4)-E_{E M}(\Delta R<0.2)}{E_{E M}(\Delta R<0.2)} .
$$

$E_{\text {total }}(\Delta R<0.4)$ is the total $(\mathrm{EM}+\mathrm{HAD})$ amount of energy found within a $\Delta R<0.4$. $E_{E M}(\Delta R<0.2)$ is the amount of EM energy found within a $\Delta R<0.2$. All initial EM clusters are required to have an isolation of less that 0.2 . The isolation parameter gives a measure of how deep and narrow a given cluster is. EM objects tend to deposit most of their energy in a narrow region of the EM layers, while hadrons deposit their energies in the hadronic layers in a much wider radius. In addition, the isolation is used to separate the electrons produced by the $Z$ decays from the EM objects produced inside jets from $\pi^{o}$ decays.

A search for a track matched to the EM object is performed by projecting the $\phi$ angle and $z$ position of the track to the third EM layer of the calorimeter, and by comparing the ratio of the transverse energy of the EM object to the transverse momentum of the track. A $\chi^{2}$ value is calculated based on Equation 5.2:

$$
\chi^{2}=\left(\frac{\Delta \phi}{\sigma_{\Delta \phi}}\right)^{2}+\left(\frac{\Delta z}{\sigma_{\Delta z}}\right)^{2}+\left(\frac{E_{T} / p_{T}-1}{\sigma_{E_{T} / p_{T}}}\right)^{2} .
$$

$\Delta \phi$ and $\Delta z$ are the azimuthal angle and z position differences between the track and EM object at the third EM layer of the calorimeter. $E_{T} / p_{T}$ is the ratio of the transverse energy of the EM object over the transverse momentum of the track. The $E_{T} / p_{T}$ term is dropped for tracks with 
$\eta$ greater than 1.1 with respect to the center of the detector. If the $\chi^{2}$ probability is greater than 0.01 then the track is considered a match with the EM object. If a track match is found, the momentum is updated using the primary vertex and the location of the track is projected to the third EM layer of the calorimeter.

Electrons deposit most of their energy in the EM layers of the calorimeter and only a small fraction will leak into the fine hadronic layers. The EM fraction is defined by the following equation:

$$
E M \text { fraction }=\frac{E_{E M}(P S+E M 1+E M 2+E M 3+E M 4)}{E_{\text {total }}(P S+\text { all EM layers }+ \text { all hadronic layers })} .
$$

The shower shape of electrons differs from the shower shape of hadrons. A covariance matrix which takes into account seven discriminant variables is formed using both test beam data and MC simulated electrons. The seven variables are:

- the individual shower energy fractions found in the four calorimeter EM layers;

- the total energy of the EM cluster;

- the position of the primary vertex;

- the cluster size in the $\mathrm{r}-\phi$ plane based on the third EM layer of the calorimeter.

The covariance matrix is defined by:

$$
M_{i j}=\frac{1}{N} \Sigma_{n=1}^{N}\left(x_{i}^{n}-\left\langle x_{i}\right\rangle\right)\left(x_{j}^{n}-\left\langle x_{j}\right\rangle\right)
$$


where $x_{i}^{n}$ is the value of variable $i$ for electron $\mathrm{n}, x_{j}^{n}$ is the value of one of the other variables $j$ for electron $\mathrm{n}$, and $\left\langle x_{i}\right\rangle$ and $\left\langle x_{j}\right\rangle$ are the average values for the two variables $i$ and $j$. The $\mathrm{H}$-matrix is the inverse of the covariance matrix $M_{i j}$. A $\chi^{2}$ variable is calculated using the H-matrix that measures the likelihood a shower shape is consistent with an EM object shower:

$$
\chi_{k}^{2}=\Sigma_{i j}\left(x_{i}^{k}-\left\langle x_{i}\right\rangle\right) H_{i j}\left(x_{j}^{k}-\left\langle x_{j}\right\rangle\right)
$$

\subsection{Jet Reconstruction and Identification}

Jets are the experimental signatures of quarks and gluons. As shown in Figure 39, there are four jet reconstruction stages: a) parton jets: clusters of quarks and gluons produced in the hard scatter; b) particle jets: clusters of particles, after the hadronization process (before hitting the detector system); c) track jets: clusters of tracks which deposited energy in the tracking system; and d) calorimeter jets: clusters of deposited energy in the calorimeter. In data, we can only measure and reconstruct track and calorimeter jets. There are two algorithms to reconstruct

jets at DØ: the Run II mid-point jet cone algorithm $(47 ; 48)$ and the $\mathrm{k}_{T}$ jet algorithm. The mid-point cone algorithm with a radius of $\Delta R<0.5$ was used in this analysis.

\subsubsection{The Jet Reconstruction Algorithm}

The midpoint cone algorithm reconstructs jets in the following steps:

- generates a list of seeds using preclustered calorimeter towers;

- forms proto-jets from the seed list;

- compiles a new seed list using the midpoints between the proto-jets; 


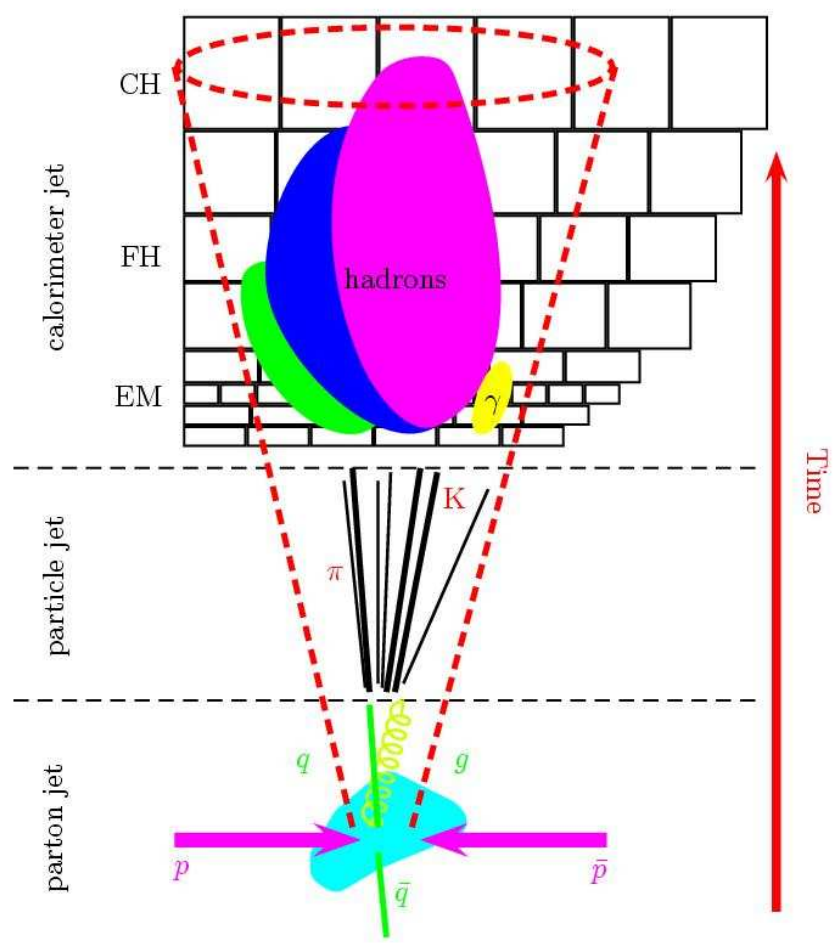

Figure 39. The decay path of quarks and gluons. Quarks and gluons after final state radiation form parton jets. Hadrons and leptons after hadronization form particle jets. Energy deposits in the calorimeter form calorimeter jets.

- forms proto-jets from the midpoint seed list;

- splits or merge overlapping jets.

Preclusters (seeds to the jet reconstruction algorithm): The energies of longitudinal calorimeter cells are added together to form calorimeter tower four vectors using the E-scheme: 


$$
p_{\text {tower }}^{\mu}=\left(E_{\text {tower }}, p_{\text {tower }}\right)=\sum_{i=0}^{\# \text { cells in tower }}\left(E_{i}, p_{i}\right) \text {. }
$$

$p_{i}$ consists of the momentum components, $p_{x}, p_{y}$, or $p_{z}$, for each calorimeter cell. $p_{i}$ is measured with respect to the primary vertex and the center of each cell. The reconstructed towers are ordered in $p_{T}$ and all towers with $p_{T}$ greater than $0.5 \mathrm{GeV}$ are used as seeds for preclustering. If the largest cell $p_{T}$ contribution in the reconstructed tower is from the coarse hadronic region, then coarse hadronic $p_{T}$ is subtracted from the reconstructed tower before requiring the $p_{T}$ of the reconstructed tower to be $>0.5 \mathrm{GeV}$ in order to become a seed for preclustering. Starting from the first seed, the next highest $p_{T}$ reconstructed tower within $\Delta R=\sqrt{(\Delta \eta)^{2}+(\Delta \phi)^{2}}<$ 0.3 is added to the cluster and the E-scheme is used to update the center of the cluster. Once a reconstructed tower is used, it is removed from the seed list. The other reconstructed towers within $\Delta R<0.3$ are added by following the same procedure. After all the clusters are formed, they are sorted in descending $p_{T}$ order, and clusters with $p_{T} \leq 1.0 \mathrm{GeV}$ are removed from the list. The calorimeter cluster list contains the seeds for the jet reconstruction algorithm.

Formation of Proto-Jets from the clustered seed list: The first seed is used to construct a proto-jet. Proto-jets have to be at least $\Delta R=\sqrt{(\Delta y)^{2}+(\Delta \phi)^{2}}>0.25$ away from each other. Rapidity is used for the rest of the cone searching algorithms, instead of pseudorapidity, which was used in the tower clustering algorithm. All the reconstructed towers within a cone of $\Delta R<0.5$ around the seed are added together via the E-scheme to form a proto-jet. Then the energy weighted center of the proto-jet is used to combine all the reconstructed towers within $\Delta R<0.5$. The reconstructed towers are added together via the E-scheme to make a 
new center. The procedure is repeated until: the $p_{T}$ of the new proto-jet is less than $4.0 \mathrm{GeV}$; the $\Delta R$ measurement refining the location of the center of the proto-jet is within 0.001 of the previous center; or the center-finding loop is repeated fifty times. The next seed from the list constructs another proto-jet. The proto-jet generating procedure repeats itself until there are no seeds left. The list of proto-jets is sorted in descending order of $p_{T}$.

Formation of Proto-Jets from midpoint seed list: Additional seeds for proto-jet generation are created by the midpoints between pairs of proto-jets using the $p_{T}$-weighted average of $(y, \phi)$. Midpoints of proto-jets are used to remove the sensitivity to soft radiation incurred by only using the clustered seed list. The midpoint between two proto-jets forms seeds as long as $0.5<\Delta R_{\text {jet,midpoint }}<1.0$. The list of midpoint seeds is passed to the proto-jet generating algorithm discussed in the previous paragraph with one exception, namely that the new proto-jets have to be within $\Delta R \leq 0.25$ of other proto-jets.

Split and Merge of Overlapping Jets: The two lists of proto-jets are merged together and sorted in order of descending $p_{T}$. Starting from the jet with the largest transverse momentum, if two jets are found to share reconstructed towers, and the jet with lower transverse momentum has more than half of its $p_{T}$ contribution due to the shared region, the two proto-jets are merged into one proto-jet. The proto-jet with the lower $p_{T}$ is removed and the leading- $p_{T}$ proto-jet is adjusted via the E-scheme using the reconstructed towers from both proto-jets. If the two proto-jets overlap and the lower $p_{T}$ proto-jet has less than half of its $p_{T}$ from the shared region, then the shared reconstructed towers are split amongst the two proto-jets. The two proto-jets divide the shared reconstructed towers based on proximity. The two proto-jets 
are re-centered based on the E-scheme. The surviving proto-jets form the final list of jets. The jets are sorted in descending order of $p_{T}$ and are required to have $p_{T}>8.0 \mathrm{GeV}$.

\subsubsection{Jet Identification}

A set of quality cut variables are used for each jet to help reduce the amount of false (fake) jets found in data due to calorimeter noise. The quality cuts also help separate electron and photon objects from jets.

EM fraction: Jets deposit a large fraction of their energy in the hadronic layers of the calorimeter. Electrons and photons, on the other hand, deposit a large fraction of their energy in the EM layers of the calorimeter. The EM fraction is defined by the following equation:

$$
\text { EM fraction }=\frac{p_{T}^{E M}}{p_{T}^{\text {total }}} .
$$

$p_{T}^{E M}$ is the amount of transverse momentum in the EM layers of the calorimeter for a given jet. $p_{T}^{\text {total }}$ is the total amount of transverse momentum of the jet.

Coarse hadronic fraction ( $\mathbf{C H F})$ : The noisiest part of the calorimeter is the coarse hadronic section. The coarse hadronic fraction is defined as the amount of transverse momentum deposited in the coarse hadronic layers divided by the total amount of transverse momentum in the jet.

$$
C H F=\frac{p_{T}^{\text {coarse hadronic layer }}}{p_{T}^{\text {total }}} .
$$


Hot Cell Fraction (HOTF): HOTF is defined as the ratio of the highest $p_{T}$ cell divided by the next highest $p_{T}$ cell in the jet. Jets with noisy calorimeter cells are removed with this cut.

n90: A jet deposits its energy over a series of calorimeter towers. Electrons/photons and single noisy (hot) towers will deposit most of their energy in one tower. N90 is the number of towers in the jet which make up $90 \%$ of the transverse momentum of the jet.

Level-1 Confirmation: To further reduce spurious jets coming from precision readout noise problems, the jet $p_{T}$ is compared to the energy found by the L1 trigger system. L1 confirmation is defined by:

$$
L 1 \text { confirm }=\frac{\text { L1set }}{p_{T}^{\text {reco }} \times(1-C H F)},
$$

where L1set is the summation of the transverse energy of the Level-1 trigger towers inside the jet. $p_{T}^{r e c o}$ is the uncorrected jet transverse momentum (before Jet Energy Scale corrections).

\subsubsection{Jet Energy Scale}

The Jet Energy Scale (JES) corrects the reconstructed energy of the jet back to the energy of the jet at the particle level, i.e. before the interaction with the detector (49). The JES corrections are applied using the formula:

$$
E^{c o r r}=\frac{E^{r e c o}-O}{R_{\text {jet }} \times S} .
$$


$\mathrm{O}$ is the offset energy due to the underlying event, multiple $p \bar{p}$ interactions, energy pile-up, electronic noise, and calorimeter noise. $R_{\text {jet }}$ is the calorimeter response to a jet which is measured using the $p_{T}$-balancing of photons and jets. $\mathrm{S}$ is a showering effect correction due to the fixed size of the cone radius and the development of the shower of particles. Particles within a jet can deposit a fraction of their energy inside a calorimeter-level cone, while nearby particles can deposit a fraction of their energy inside the same calorimeter-level cone. The JES is determined for data and MC events separately. The JES corrections for data are shown in Figure 40.

In addition to the JES corrections, jets are corrected for muon decays inside the jet. Some jets have a large fraction of their energy in leptonic decay products. The muons only deposit a small fraction of their energy in the calorimeter, while the associated neutrinos do not deposit any energy in the calorimeter. The estimated energy of the muons is added back into the jets.

\subsection{Muon Reconstruction}

Muons produced in semi-leptonic decays of B hadrons are used in this analysis as an additional constraint when tagging b-quark initiated jets. The muons are reconstructed (50) by the muon detectors using hit information from the A and BC layers. Hits in these layers form segments. A muon is identified when it has at least two wire hits and one scintillator hit in the A layer, and at least two wire hits and one scintillator hit in the BC layers. The momentum of the muon is calculated by combining segments from the A region and $\mathrm{BC}$ layers using the curvature of the muon paths. Once a muon is identified, a search in $(\Delta \theta, \Delta \phi)$ of the projected location of the muon is conducted in the tracking system. If multiple tracks are found, the track 

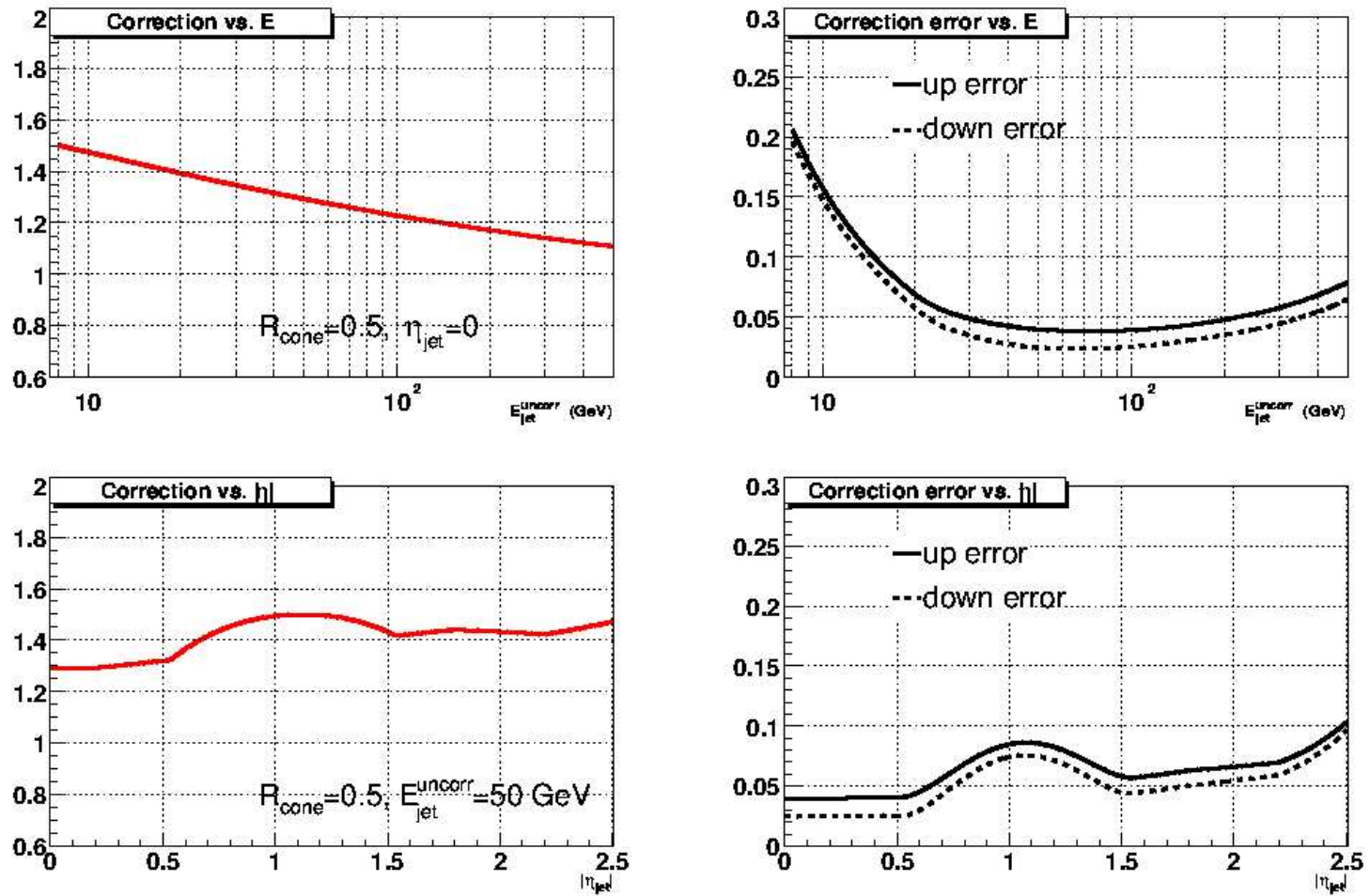

Figure 40. The jet energy scale corrections, left, and uncertainties, right, for reconstructed calorimeter jets measured in data.

with the lowest $\chi_{\text {track }}^{2}$ value is used as the track match. The momentum vector of the muon is recalculated based on the momentum vector of the track. Cosmic ray muons are rejected using timing information from the scintillator hits. The muons used in this analysis have $p_{T}>4.0$ $\mathrm{GeV}$, IP (impact parameter) along the $\mathrm{z}$ axis $<1 \mathrm{~cm}$ from the primary vertex, and a match to a jet within $\Delta R_{\text {muon }, \text { jet }}<0.5$. 


\section{$5.6 \quad$ b-jet Tagging}

b quarks produced from the $p \bar{p}$ collisions will hadronize into B baryons and mesons. $\mathrm{B}$ hadron lifetimes are on the order of $1.6 \mathrm{ps}$. Therefore, a $40 \mathrm{GeV}$ B hadron will travel $3 \mathrm{~mm}$ from the primary vertex before decaying. The decay will produce tracks which will point back to a secondary vertex instead of the primary vertex. The tracks that are found near $(\Delta R$ $<0.5)$ a calorimeter jet are used to tag b jets. The impact parameters and uncertainties of tracks associated with a calorimeter jet are used to tag b jets from light-quark jets $(\mathrm{u}, \mathrm{d}, \mathrm{s})$. The b-tagging algorithm used in this analysis is the jet lifetime probability (JLIP) algorithm (51; 52). JLIP is based on the probability that the jet was produced from a light quark. The JLIP algorithm is applied to "taggable" jets. A taggable jet has tracks from the central tracking system which point in the same direction as the calorimeter jet.

\subsubsection{Taggable Jets}

Jet "taggability" is a track confirmation quality cut (53). A taggable jet is required to have a match with a "track jet" within $\Delta R<0.5$. A "track jet" is reconstructed using a simple cone clustering algorithm with a cone radius of 0.5 using the following criteria:

- at least one seed track with $p_{T}>1 \mathrm{GeV}$;

- at least one additional track with $p_{T}>0.5 \mathrm{GeV}$;

- each track in the cluster should have:

- at least 1 SMT hit (using SMT ladders + F disk wedges);

- dca (distance of closest approach in x-y plane) $<0.2 \mathrm{~cm}$; 
- zdca (distance of closest approach along the $\mathrm{z}$ axis) $<0.4 \mathrm{~cm}$;

$-\Delta z<2.0 \mathrm{~cm}$ to the current track-jet $\mathrm{z}$ position (the track-jet direction and z position are updated after the addition of each track).

\subsubsection{Jet LIfetime Probability (JLIP)}

The JLIP algorithm utilizes all tracks associated with a jet. The IPs for tracks decaying from B mesons, with respect to the primary vertex, are on the order of $400 \mu \mathrm{m}$. The algorithm uses the IP of the tracks with respect to the primary vertex, IP errors, and projections along the jet axis to determine the probability that each track originated from the primary vertex. JLIP is the multiplication of the individual probabilities of the tracks associated with a jet. Jets from light quarks have a flat JLIP distribution between 0 and 1 , while jets from b quarks have preferentially low probability values, close to 0 . The efficiency of the JLIP algorithm is defined as the ability to "tag" a b jet given a taggable b jet.

The tracks used in the JLIP algorithm must satisfy the following criteria:

- track within $\Delta R<0.5$ of the jet axis;

- $\operatorname{track} p_{T}>1 \mathrm{GeV}$;

- distance of closest approach $<0.2 \mathrm{~cm}$ in the $\mathrm{x}-\mathrm{y}$ plane;

- distance of closest approach $<0.4 \mathrm{~cm}$ along the $\mathrm{z}$ axis;

- at least 1 SMT hit. 
An impact parameter significance value $\left(\mathrm{S}_{I P}\right)$ is calculated for each track. $\mathrm{S}_{I P}$ is defined as the impact parameter of the track divided by the uncertainty of the impact parameter measurement:

$$
S_{I P}=\frac{I P}{\sigma_{I P}}
$$

The impact paramter has a sign based on the projection of the impact parameter vector onto the jet axis vector. b-quark jets have more tracks with large positive $\mathrm{S}_{I P}$ than light-quark jets. Twenty nine $\mathrm{S}_{I P}$ distributions, described in 5.6.1.1, are formed by grouping tracks together which have similiar characteristics: $p_{T}, \eta, \chi^{2}$, and number of hits.

\begin{tabular}{|ccccc|}
\hline SMT hits & CFT hits & $|\eta|$ & $\chi^{2}$ & $p_{T}$ \\
\hline$\geq 1$ hit in inner layer & $\leq 6$ & $1.6-2.0$ or $>2.0$ & $>0$ & $>1$ \\
\hline 1 superlayer & $\geq 7$ & $\leq 1.2$ & $>0$ & $>1$ \\
1 superlayer & $\geq 7$ & $\geq 1.2$ & $0-2$ or $>2$ & $>1$ \\
\hline 2,3 , or 4 superlayers & $\geq 7$ & $\leq 1.2$ & $0-2$ & $1-2,2-4$, or $>4$ \\
2,3 , or 4 superlayers & $\geq 7$ & $\leq 1.2$ & $2-4$ or $>4$ & $>1$ \\
2,3, or 4 superlayers & $\geq 7$ & $1.2-1.6$ & $0-2$ or $>2$ & $>1$ \\
2,3, or 4 superlayers & $\geq 7$ & $\geq 1.6$ & $>0$ & $>1$ \\
\hline
\end{tabular}

TABLE VII

TRACK CATEGORIES USED FOR THE PARAMETERIZATION OF THE IMPACT PARAMETER RESOLUTION FUNCTIONS. 
The uncertainty on the track impact parameter measurement $\left(\sigma_{I P}^{\text {raw }}\right)$ is corrected by a pull value. The pull value takes into account uncertainties due to the number of tracks associated with the primary vertex, the pseudorapidity of the track (which accounts for smearing due to multiple scattering), and the number of SMT and CFT hits assigned to the track. The $\mathrm{S}_{I P}$ functions (Figure 41) are fit with a Gaussian distribution, centered at $S_{I P}=0$, and convoluted with an exponential distribution to model the tails. The negative side of the $S_{I P}$ distributions have equivalent distributions for light jets and $\mathrm{b}$ jets. Therefore, the negative side is used to form impact parameter resolution functions $\left(\mathrm{R}\left(S_{I P}\right)\right)$ parameterizations shown in Figure 42. The $\mathrm{R}\left(S_{I P}\right)$ parameterizations are based on the twenty-nine different $S_{I P}$ distributions.

The $\mathrm{R}(\mathrm{s})$ functions are used to calculate the probability that a track originated from the primary vertex $\mathrm{P}_{t r k}\left(S_{I P}\right)$ (Figure 43$) . \mathrm{P}_{t r k}\left(S_{I P}\right)$ is defined as the area of the $\mathrm{R}\left(S_{I P}\right)$ distribution, up to the tracks $S_{I P}$ value, divided by the total area of the $\mathrm{R}\left(S_{I P}\right)$ distribution:

$$
P_{t r k}\left(S_{I P}\right)=\frac{\int_{-50}^{-\left|S_{I P}\right|} R\left(S_{I P}\right) d s_{I P}}{\int_{-50}^{0} R\left(S_{I P}\right) d s_{I P}}
$$

Tracks with $S_{I P}$ values close to zero will have $\mathrm{P}_{t r k}$ values close to one. The jet lifetime probability is calculated by multiplying all track probabilities, $\mathrm{P}_{\text {trk }}$, together:

$$
P_{j e t}=\Pi_{i=1}^{N_{t r k}} P_{t r k}\left(S_{I P}\right)_{i} \times \Sigma_{j=0}^{N_{t r k}-1} \frac{\left(-\ln \left(\Pi_{i=1}^{N_{t r k}} P_{t r k}\left(S_{I P}\right)_{i}\right)\right)^{j}}{j !} .
$$

Figure 44 shows that light jets have a flat JLIP distribution while the $\mathrm{b}$ and $\mathrm{c}$ jets peak at zero. The mis-tag probability, tagging a light-quark jet as a b jet, is roughly equivalent to the JLIP 
working point. Therefore, a JLIP cut value of four percent has a mis-tag rate of four percent. Hadrons produced from c-quark decays can also travel a measurable distance from the primary vertex before decaying. The efficiency of tagging a $\mathrm{c}$ jet is approximately half the b-tagging efficiency value given that the taggable $\mathrm{c}$ jet has equivalent $p_{T}$ and $\eta$. 

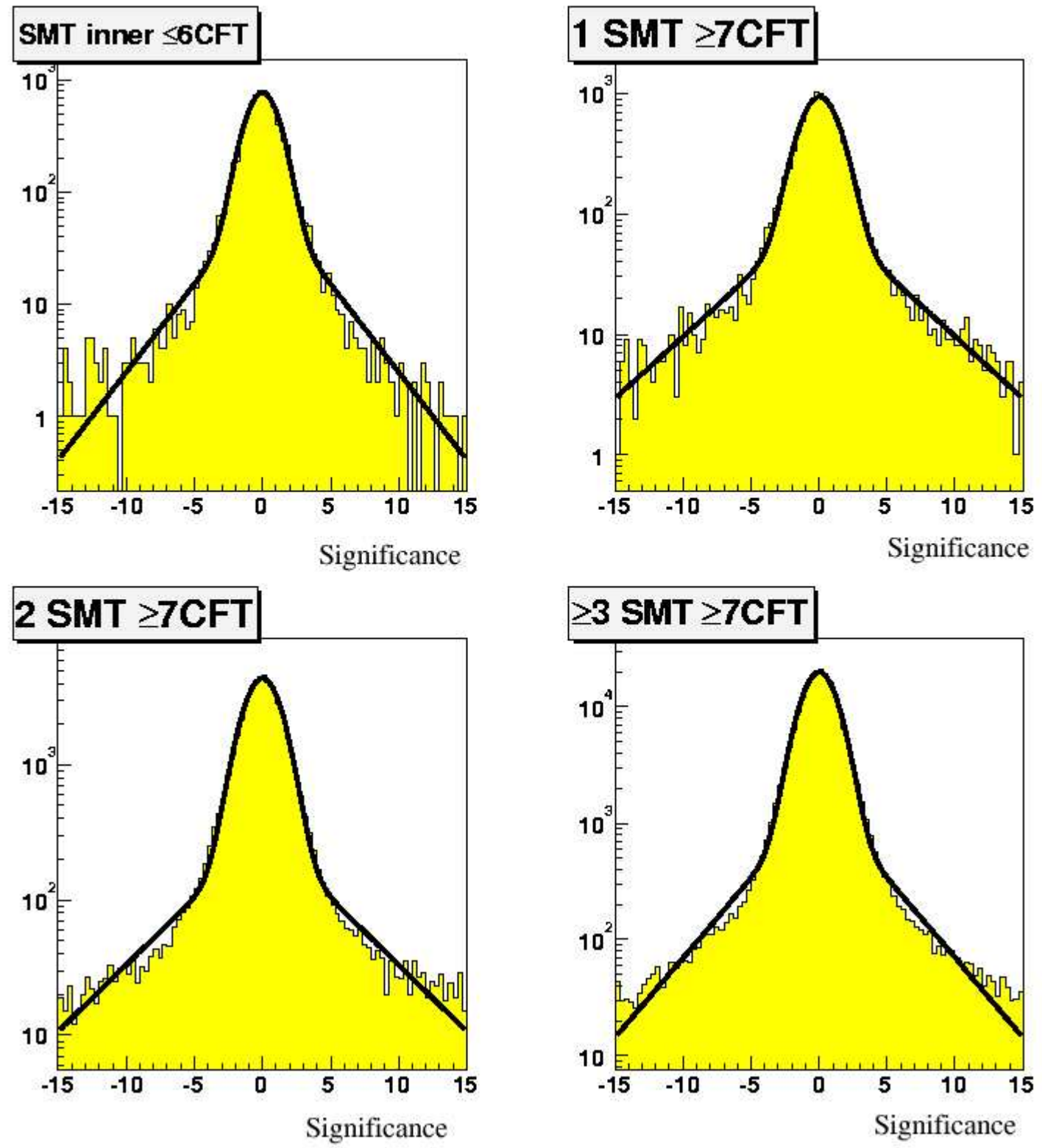

Figure 41. Impact parameter significance $\frac{I P}{\sigma}$ for tracks measured in data using four different track selections based on the number of hits in the SMT and CFT detectors. The peak is fitted with a Gaussian function while the tails are fitted with an exponential function. 

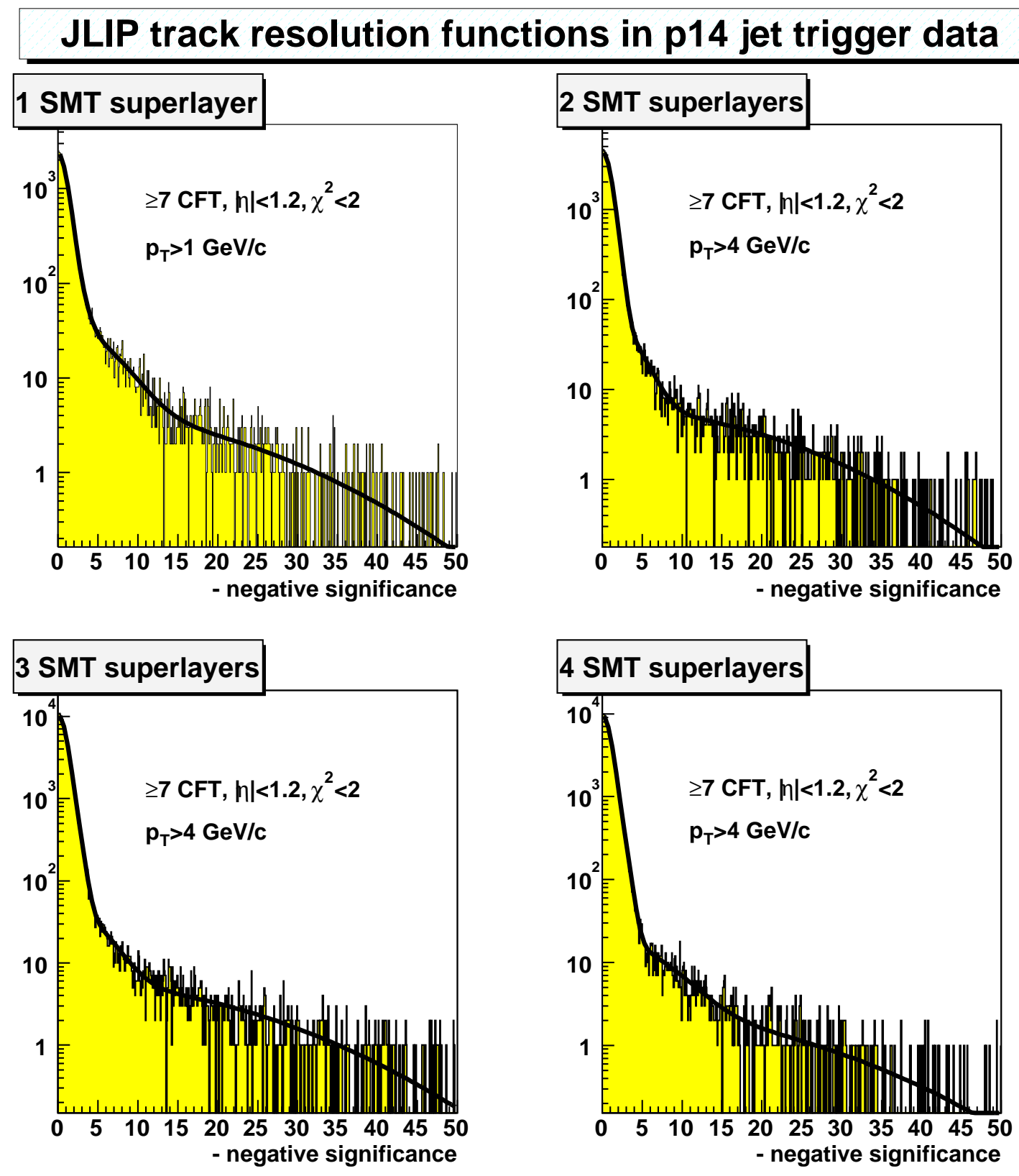

Figure 42. Resolution R(s) distributions using the negative side of the IP significance curves. There are 29 resolution fits. Four of the fits are shown above. The resolution distributions are fit by adding four Gaussian functions together. 


\section{track significance probability}
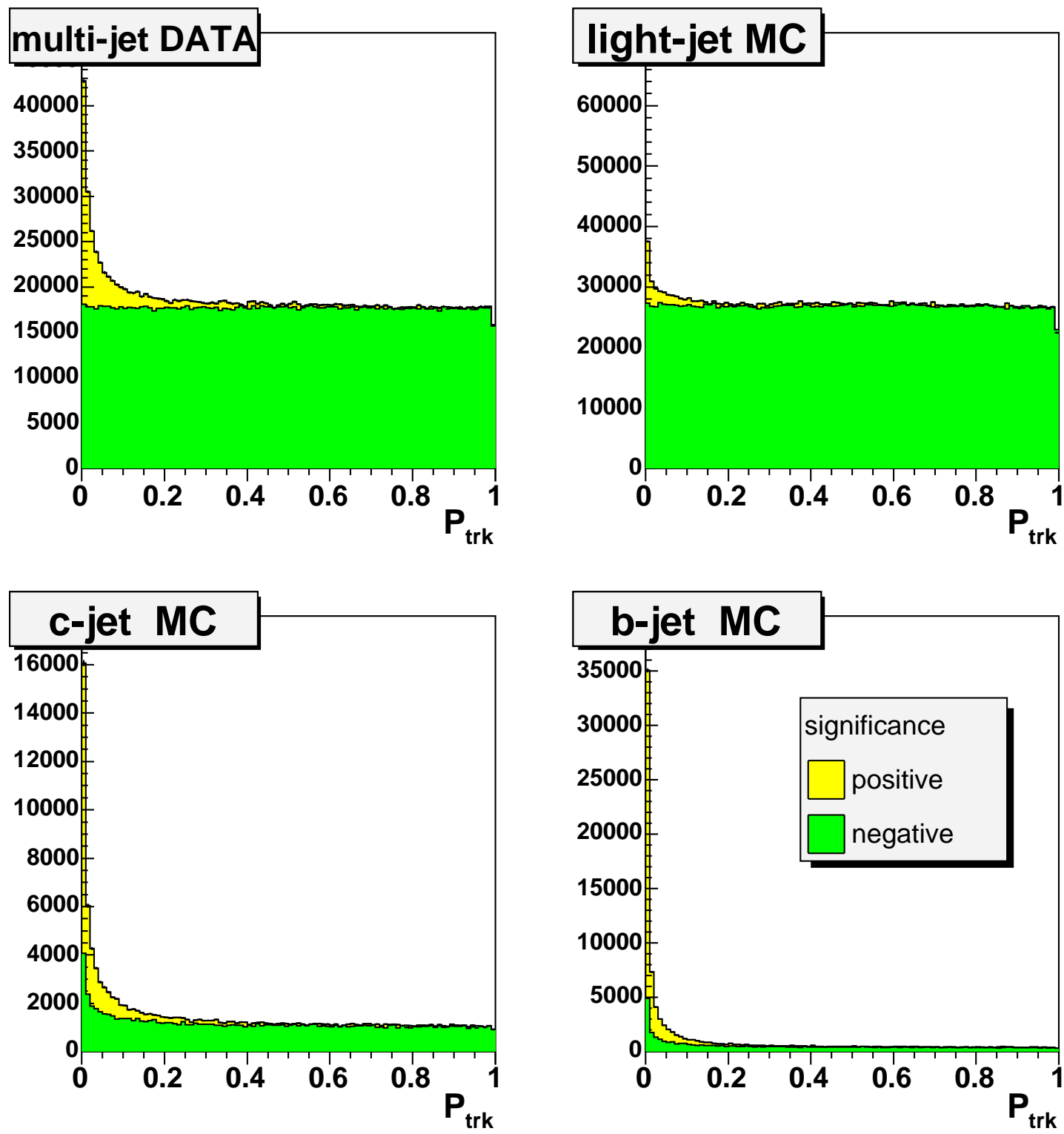

Figure 43. Using the resolution functions from the previous figure, a track probability is calculated using only positive sign IP significance tracks. 


\section{Jet Llfetime Probability}
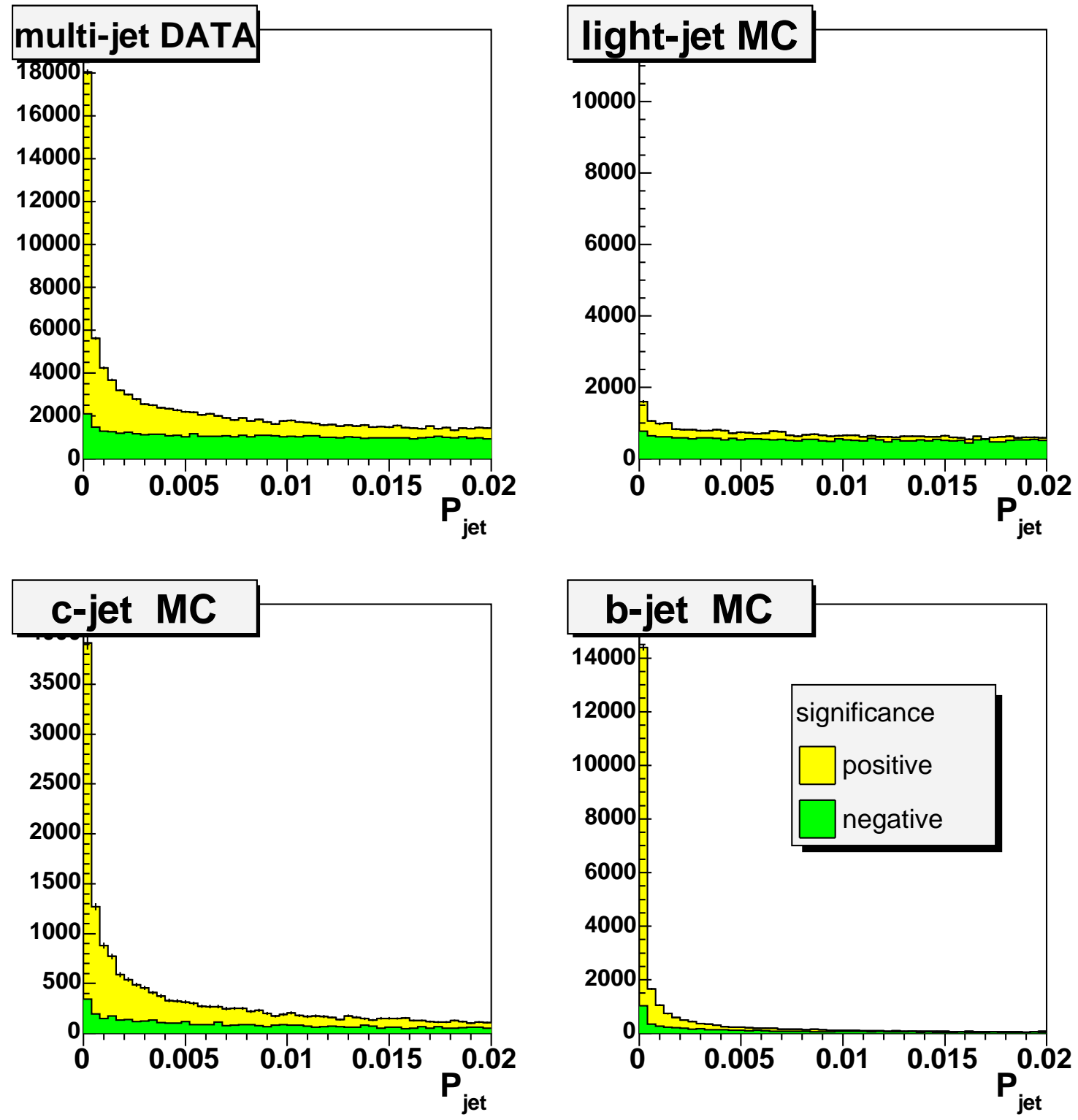

Figure 44. Combining all the positive sign IP significance tracks generate the JLIP probability. The upper left plot shows that using the negative signed $S_{I P}$ tracks gives a flat distribution for a b-tagged enhanced data sample. The upper right plot shows a flat probability distribution for light jets from MC. The bottom right plot shows that b jets in MC are highly peaked at low JLIP probabilities. 


\section{CHAPTER 6}

\section{MONTE CARLO EVENT SIMULATION}

Monte Carlo (MC) event generators simulate high-energy physics events to provide a representation of nature that is as accurate as possible. The event generators simulate all stages of interactions that occur in high energy collisions: the initial-state radiation; the hard scattering of partons; the final-state radiation; the hadronization of partons, and the interaction of final particles with the detector elements. Simulated MC events allow the estimation of acceptances, the optimization of selection cuts, and the direct comparison of theoretical predictions to data. Three MC event generators are used in this analysis: PythiA (54), ALPGEN (55), and Monte Carlo for FeMtobarn processes (MCFM) (56). This chapter describes the MC simulation tools and techniques used in this dissertation.

\subsection{The PYTHIA Event Generator}

PYTHIA is a general purpose event generator which can model 240 different hard processes. The hard processes are described as $2 \rightarrow 2$ parton scatterings. The event generator contains a simulation of several physics aspects of the proton-antiproton interaction:

- The initial beam particles, such as protons and antiprotons, are characterized by parton distribution functions (PDF);

- Incoming partons from each initial proton (antiproton) radiate gluons which form the initial-state radiation; 
- Two incoming partons participate in the hard process which produces two outgoing partons;

- The hard process may produce a set of short-lived resonances like the $\mathrm{W}^{ \pm}$or $\mathrm{Z}^{o}$ boson;

- The final state partons radiate gluons which form the final-state radiation;

- Initial and final-state color coherence effects are incorporated via the Angular Ordering approximation;

- The remaining partons in the two incoming hadrons which did not participate in the hard process form the beam remnants that populate the forward and backward rapidities;

- Outgoing quarks and gluons fragment to color neutral hadrons following the string fragmentation;

- Many of the produced hadrons are unstable and decay further.

The PDFs give the probability for a parton to carry a fraction $\mathrm{x}$ of the proton or antiproton total momentum. The PDFs, a function of $\mathrm{x}$ and $\mathrm{Q}^{2}$, are based on global fits to data (57). The initial and final state partonic evolution is based on the Dokshitzer-Gribov-Lipatov-Altarelli-Parisi (DGLAP) equations $(58 ; 59 ; 60)$ which give the probability for a parton to radiate. The final state parton shower is evolved from the scale $\mathrm{Q}$ down to a cut-off scale $\mathrm{Q}_{o}$ where it terminates. Outgoing quarks and gluons form color neutral hadrons following the string hadronization model. The string hadronization model starts with a gluon forming a $q \bar{q}$ pair traveling in opposite directions in which a string is attached to the pair. As the pair travels farther apart, the string increases in potential energy until enough energy is gathered to break the string by 
pulling a $q \bar{q}$ pair from the vacuum to form a colorless hadron. This string hadronization process continues until only on mass-shell hadrons remain. Although PYTHIA allows events with high jet multiplicities from initial and final state radiations, these soft and collinear jets do not model high jet multiplicity events well.

\subsection{The ALPGEN Event Generator}

ALPGEN is a multiparton hard-process event generator which performs, at leading order in QCD and EW interactions, the calculation of exact matrix elements for a large set of parton-level processes. The event generator can generate final states with several hard and well separated jets. Since ALPGEN is a tree-level matrix element generator, a treatment of higher-order corrections leading to the development of partonic cascades and the transformation of partons into observables is needed. The ALPGEN output is interfaced with PYTHIA which provides the partonic showering and hadronization.

\subsection{MCFM Simulation}

MCFM can calculate parton level cross sections for up to $Z / \gamma^{*}+2$ parton processes at next-to-leading order in $\alpha_{s}$.

\subsection{DØ Detector Simulator}

After all final state particles are generated, detector effects can be simulated with a simulation package called DØGSTAR (DØ GEANT Simulation of the Total Apparatus Response) (61). DØGSTAR is based on the CERN package GEANT (GEometry ANd Tracking) (62) which simulates the passage of particle through matter. Effects such as ionization, showering, and the magnetic field interaction in the $\mathrm{D} \varnothing$ detector are modeled. A package called DØsim 
(63) provides the effects of sampling electronics, calorimeter pile-up from previous events, and noise and detector losses from the SMT, CFT, calorimeter, and muon detector systems. For direct comparisons, DØsim produces the simulated output in the same format as data. 


\section{CHAPTER 7}

\section{DATA AND MC SAMPLES}

\subsection{Data Sample}

The data sample used in this analysis was collected between April 2002 and August 2004. Events from $Z / \gamma^{*} \rightarrow e^{+} e^{-}$decays were selected online with a combination of single-electron triggers based on energy deposited in the calorimeter trigger towers. Each raw data event was reconstructed using the p14 version of the $\mathrm{D} \varnothing$ reconstruction software with a calorimeter noise suppression algorithm (T42 (64)) applied, and then skimmed down into the EM1TRK skim. The 64 million events which enter the EM1TRK skim have: at least one EM object with ID $=10$ or $\pm 11, p_{T}>8 \mathrm{GeV}$, and a track with $p_{T}>5 \mathrm{GeV}$ within $\Delta \phi=0.1$ of the EM object. Athena (65) p16.06.00 with DØcorrect tag p16-br-07 v8.2 and JES v5.3 was used to produce the analysis root-tuples.

Using the run quality database, runs for which the SMT, CFT, and Calorimeter sub-systems were flagged as "bad" were excluded from the analysis. Events that were flagged as "bad" by the luminosity or CalJetMet group were excluded by LBN. Furthermore, runs with limited L1Cal trigger coverage, $\left|\eta_{\text {det }}\right|^{1}<0.8$, were excluded. If an event had a reconstructed di-em invariant mass around the $Z$ boson mass, but failed the calorimeter event quality (66) criteria,

\footnotetext{
${ }^{1}$ Pseudorapidity, $\eta_{\text {det }}$, is calculated with respect to the center of the detector.
} 
the LBN corresponding to the event was excluded. A summary of all LBNs and events that were excluded is found in (67).

Events for the analysis are selected using triggers that required at least one EM object. Table VIII lists the single-electron trigger names, and Table IX shows the L1, L2, and L3 composition of these triggers. Table $\mathrm{X}$ describes the L1, L2, and L3 trigger terms. The data sample is grouped into similar trigger periods corresponding to: v8-10, v11, v12, and v13 trigger lists. The luminosity was calculated using the trigger mapping scheme. Based on these triggers, the measured luminosity is $L=452 \mathrm{pb}^{-1}(68)$ with a $6.5 \%$ uncertainty $(69 ; 70)$.

The trigger mapping scheme only uses unprescaled triggers and the triggers are examined with the priority order shown in Table VIII. The first group of triggers in the trigger map table indicates that if an event has all four of these triggers unprescaled, and if any of the four triggers pass, the event is kept in the analysis. Otherwise the next set of triggers are examined. 


\begin{tabular}{|c|}
\hline \multicolumn{2}{|c|}{ Trigger Name } \\
\hline E1_SHT20 $\mid$ E2_SHT20 $\mid$ E3_SHT20 $\mid$ E1_SH30 all unprescaled \\
E1_SHT20 $\mid$ E2_SHT20 $\mid$ E1_SH30 all unprescaled \\
E1_SHT20 | E1_SH30 all unprescaled \\
E1_SHT20 \\
E1_SHT22 | E2_SHT22 | E3_SHT22 $\mid$ E1_SH30 all unprescaled \\
E1_SHT22 $\mid$ E2_SHT22 $\mid$ E1_SH30 all unprescaled \\
E1_SHT22 | E1_SH30 all unprescaled \\
E1_SHT22 \\
EM_HI_SH | EM_HI_2EM5_SH all unprescaled \\
EM_HI_SH \\
EM_HI \\
EM_MX_SH \\
EM_MX
\end{tabular}

TABLE VIII

TRIGGERS USED IN THE ANALYSIS.

\begin{tabular}{|llll|}
\hline Trigger & L1 & L2 & L3 \\
\hline E1_SHT20 & CEM $(1,11)$ & EM $(1,15)$ v13 only & ELE_NLV_SHT $(1,20)$ \\
E2_SHT20 & $\operatorname{CEM}(2,6)$ & EM $(1,15)$ v13 only & ELE_NLV_SHT $(1,20)$ \\
E3_SHT20 & $\operatorname{CEM}(1,9) \operatorname{CEM}(2,3)$ & EM $(1,15)$ v13 only & ELE_NLV_SHT $(1,20)$ \\
E1_SH30 & CEM $(1,11)$ & EM $(1,15)$ v13 only & ELE_NLV_SH $(1,30)$ \\
E1_SHT22 & CEM $(1,11)$ & EM $(1,15)$ & ELE_NLV_SHT $(1,22)$ \\
E2_SHT22 & CEM $(2,6)$ & EM $(1,15)$ & ELE_NLV_SHT $(1,22)$ \\
E3_SHT22 & CEM $(1,9) C E M(2,3)$ & EM $(1,15)$ & ELE_NLV_SHT $(1,22)$ \\
EM_HI_SH & CEM $(1,10)$ & EM $(1,12)$ & ELE_LOOSE_SH_T $(1,20)$ \\
EM_HI_2EM5_SH & CEM $(2,5)$ & EM $(1,12)$ & ELE_LOOSE_ST_T $(1,20)$ \\
EM_HI & CEM $(1,10)$ & EM $(1,12)$ & ELE_LOOSE $(1,30)$ \\
EM_MX_SH & CEM $(1,15)$ & none & ELE_LOOSE_SH_T $(1,20)$ \\
EM_MX & CEM $(1,15)$ & none & ELE_LOOSE $(1,30)$ \\
\hline
\end{tabular}

TABLE IX

THE TRIGGER NAMES BROKEN UP INTO THEIR CORRESPONDING L1, L2, AND L3 TRIGGER TERMS. 


\section{L1 Triggers}

\begin{tabular}{|c|c|}
\hline $\operatorname{CEM}(1,11)$ & one EM trigger tower with $E_{T}>11 \mathrm{GeV}$ \\
\hline $\operatorname{CEM}(2,6)$ & two EM trigger towers with $E_{T}>6 \mathrm{GeV}$ \\
\hline $\operatorname{CEM}(1,9) \operatorname{CEM}(2,3)$ & $\begin{array}{l}\text { one EM trigger tower with } E_{T}>9 \mathrm{GeV} \text {, } \\
\text { another EM trigger tower with } E_{T}>3 \mathrm{GeV}\end{array}$ \\
\hline $\operatorname{CEM}(1,10)$ & one EM trigger tower with $E_{T}>10 \mathrm{GeV}$ \\
\hline $\operatorname{CEM}(2,5)$ & two EM trigger tower with $E_{T}>5 \mathrm{GeV}$ \\
\hline $\operatorname{CEM}(1,15)$ & one EM trigger towers with $E_{T}>15 \mathrm{GeV}$ \\
\hline \multicolumn{2}{|r|}{ L2 Triggers } \\
\hline $\operatorname{EM}(1,15)$ & one EM candidate with $E_{T}>15 \mathrm{GeV}$ \\
\hline $\operatorname{EM}(1,12)$ & one EM candidate with $E_{T}>12 \mathrm{GeV}$ \\
\hline \multicolumn{2}{|r|}{ L3 Triggers } \\
\hline ELE_NLV_SHT $(1,20)$ & $\begin{array}{l}\text { one electron with }|\eta|<3.6 \text { and } E_{T}>20 \mathrm{GeV} \text { passing } \\
\text { tight shower shape cuts }\end{array}$ \\
\hline ELE_NLV_SH(1,30) & $\begin{array}{l}\text { one electron with }|\eta|<3.6 \text { and } E_{T}>30 \mathrm{GeV} \text { passing } \\
\text { loose shower shape cuts }\end{array}$ \\
\hline ELE_NLV_SHT $(1,22)$ & $\begin{array}{l}\text { one electron with }|\eta|<3.6 \text { and } E_{T}>22 \mathrm{GeV} \text { passing } \\
\text { tight shower shape cuts }\end{array}$ \\
\hline ELE_LOOSE_SH_T $(1,20)$ & $\begin{array}{l}\text { one electron with }|\eta|<3.0 \text { and } E_{T}>20 \mathrm{GeV} \text { passing } \\
\text { loose requirements including shower shape cuts }\end{array}$ \\
\hline ELE_LOOSE $(1,30)$ & $\begin{array}{l}\text { one electron with }|\eta|<3.0 \text { and } E_{T}>30 \mathrm{GeV} \text { passing } \\
\text { loose requirements }\end{array}$ \\
\hline
\end{tabular}

TABLE X

L1, L2, AND L3 TRIGGERS USED IN THIS ANALYSIS. 


\subsection{Monte Carlo Samples}

The MC samples, which are used to estimate signal acceptance and the background contributions, are summarized in Table XI. All these samples are passed through full detector simulation. A pythia $Z / \gamma^{*} \rightarrow e^{+} e^{-}$sample is used for efficiency studies. For background estimation studies, events are generated with ALPGEN and passed through PYTHIA for parton showering and hadronization. PYTHIA $Z H \rightarrow e^{+} e^{+} b \bar{b}$ samples are used to estimate the signal efficiency.

\begin{tabular}{|c|c|c|c|}
\hline Process & Generator & $\sigma \times \mathrm{Br}(\mathrm{pb})$ & \# of events \\
\hline $\mathrm{ZH} \rightarrow e \bar{e} b \bar{b} \quad M_{H}=105 \mathrm{GeV}$ & PYTHIA & 0.0040 & 5000 \\
\hline $\mathrm{ZH} \rightarrow e \bar{e} b \bar{b} M_{H}=115 \mathrm{GeV}$ & PYTHIA & 0.0028 & 5000 \\
\hline $\mathrm{ZH} \rightarrow e \bar{e} b \bar{b} M_{H}=125 \mathrm{GeV}$ & PYTHIA & 0.0018 & 5000 \\
\hline $\mathrm{ZH} \rightarrow e \bar{e} b \bar{b} M_{H}=135 \mathrm{GeV}$ & PYTHIA & 0.0011 & 5000 \\
\hline $\mathrm{ZH} \rightarrow e \bar{e} b \bar{b} M_{H}=145 \mathrm{GeV}$ & PYTHIA & 0.0005 & 5000 \\
\hline $\mathrm{Zbb} \rightarrow e \bar{e} b \bar{b}$ & ALPGEN+PYTHIA & 0.513 & 98000 \\
\hline$t \bar{t} \rightarrow 2 b+2 l$ & ALPGEN+PYTHIA & 0.671 & 154000 \\
\hline$t \bar{t} \rightarrow 2 b+1 l+2 j$ & ALPGEN+PYTHIA & 2.676 & 314050 \\
\hline$Z Z \_i n c l$ & PYTHIA & 1.56 & 107000 \\
\hline$W Z \_i n c l$ & PYTHIA & 3.68 & 203250 \\
\hline$Z j \bar{j} \rightarrow e \bar{e} j \bar{j}$ & ALPGEN+PYTHIA & 29.4 & 272450 \\
\hline$Z \rightarrow e \bar{e}+X$ & PYTHIA & 266.7 & 400000 \\
\hline
\end{tabular}

TABLE XI

SUMMARY OF MONTE CARLO SAMPLES USED IN THIS ANALYSIS. 
The energy resolution of electrons in data is not correctly modeled by the MC simulation. Additional energy smearing is applied to the MC electrons to account for the difference. $p_{x}$, $p_{y}, p_{z}$, and the energy of the electrons are multiplied by $c \cdot \operatorname{Gauss}(1, \mathrm{f})$, where $\operatorname{Gauss}(1, \mathrm{f})$ is a smearing parameter, which is chosen from a Gaussian distribution with mean of 1, a width of $f$, and an overall calibration factor of c. For this analysis, the following values for the smearing parameters are used (71):

for central electrons $\left(\left|\eta_{\text {det }}\right|<1.1\right)$ :

- $f=0.045$

- $c=1.003$

for forward electrons $\left(\left|\eta_{\text {det }}\right|>1.1\right)$ :

- $f=0.034$

- $c=0.996$

The energy resolution of jets in data (Figure 45) is not correctly described by the MC simulation (Figure 46), and additional jet energy resolution smearing is applied to the MC jets (72). Equation 7.1 shows the energy resolution parameterization where N, S, and C denote the noise, sampling, and constant gain fluctuation terms, respectively.

$$
\frac{\sigma\left(p_{T}\right)}{p_{T}}=\sqrt{\frac{N^{2}}{p_{T}^{2}}+\frac{S^{2}}{p_{T}}+C^{2}}
$$

Table XII summarizes all coefficients for different detector regions. For a given jet, if the data resolution is better than the MC resolution, no additional smearing is applied. If the jet energy 
resolution is worse in data than in $\mathrm{MC}$, a multiplicative smearing factor (Equation 7.2) is applied to the components of the momentum four-vector of each jet.

$$
\text { Smearing Factor }=\text { Gauss }\left(1, \sqrt{\left(\frac{\sigma\left(p_{T}\right)}{p_{T}}\right)_{\text {data }}^{2}-\left(\frac{\sigma\left(p_{T}\right)}{p_{T}}\right)_{M C}^{2}}\right)
$$

\begin{tabular}{|c|c|c|c|c|}
\hline Coefficient & $\left|\eta_{\text {det }}\right|<0.5$ & $0.5<\left|\eta_{\text {det }}\right|<1.0$ & $1.0<\left|\eta_{\text {det }}\right|<1.5$ & $\left|\eta_{\text {det }}\right|>1.5$ \\
\hline$N_{\text {data }}$ & 5.05 & $9.06 \cdot 10^{-9}$ & 2.24 & 6.42 \\
\hline$S_{\text {data }}$ & 0.753 & 1.2 & 0.924 & 0 \\
\hline$C_{\text {data }}$ & 0.0893 & 0.087 & 0.135 & 0.0974 \\
\hline$N_{M C}$ & 4.26 & 4.61 & 3.08 & 4.83 \\
\hline$S_{M C}$ & 0.658 & 0.621 & 0.816 & 0 \\
\hline$C_{M C}$ & 0.0436 & 0.0578 & 0.0729 & 0.0735 \\
\hline
\end{tabular}

TABLE XII

JET RESOLUTION PARAMETERS IN DATA AND MC. 

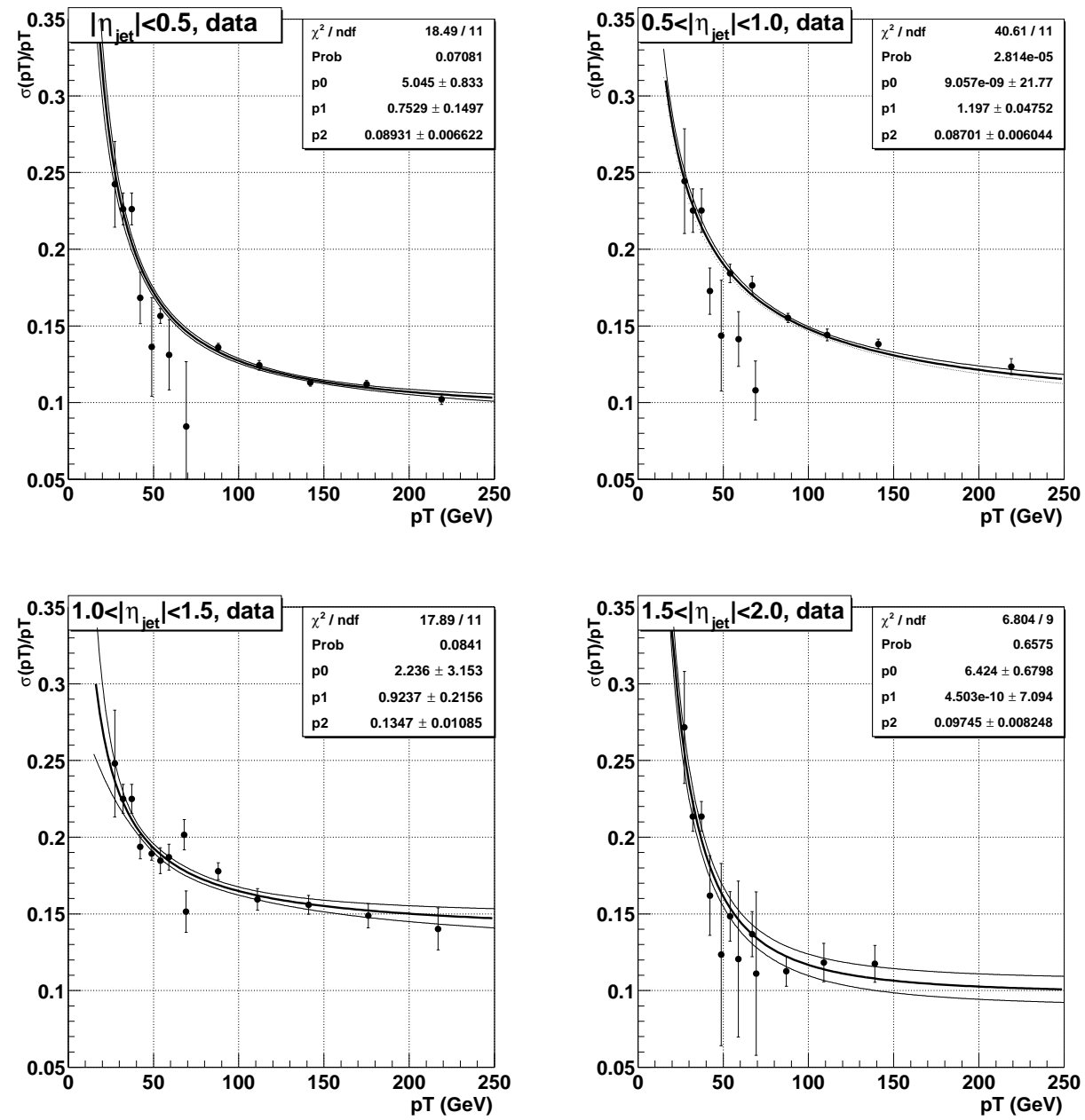

Figure 45 . Jet $p_{T}$ resolutions for different $\eta_{\text {det }}$ regions in data. The points below $\sim 50 \mathrm{GeV}$ are obtained using $\gamma+$ jet events, whereas for $p_{T} \geq 50 \mathrm{GeV}$ resolutions are measured using di-jet events. Bands of $\pm 1 \sigma$ statistical error are also shown. 

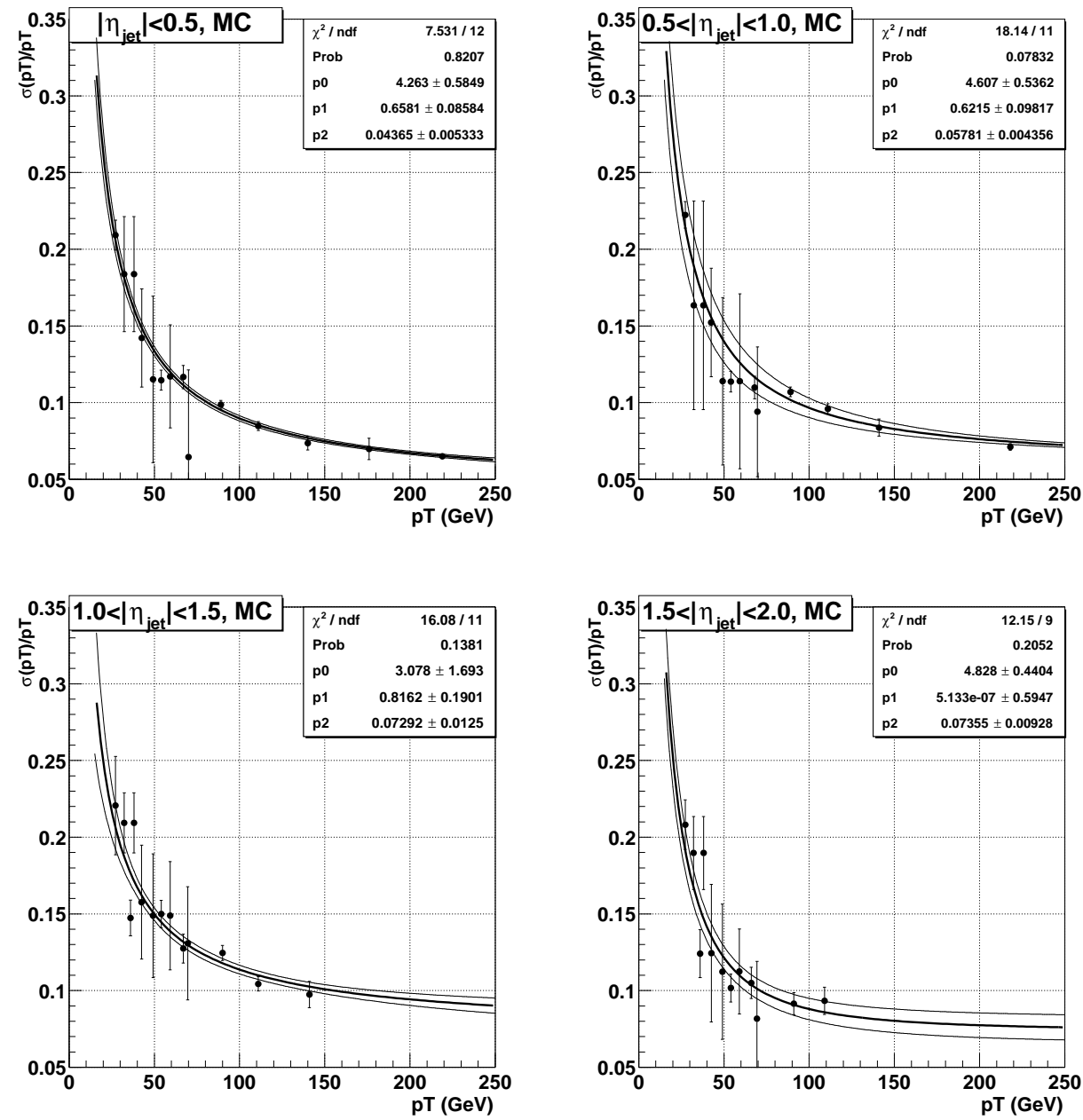

Figure 46 . Jet $p_{T}$ resolutions for different $\eta_{\text {det }}$ regions in $\mathrm{MC}$. The points below $\sim 50 \mathrm{GeV}$ are obtained using $\gamma+$ jet events, whereas for $p_{T} \geq 50 \mathrm{GeV}$ resolutions are measured using di-jet events. Bands of $\pm 1 \sigma$ statistical error are also shown. 


\subsection{Event Selection}

The following selection criteria are applied in order to assure that events with two high $p_{T}$ electrons originating from the decay of a $Z / \gamma^{*}$ gauge boson are selected. After identifying the $Z$ candidate events, the presence of at least two high $p_{T}$ jets with only one (or two) of these jets tagged as b jets is required.

\subsubsection{Primary Vertex}

The efficiency to reconstruct the PV is nearly $100 \%$ in the central region of the detector, and decreases outside of the SMT fiducial volume. Therefore, the PV is required to be within $60 \mathrm{~cm}$ of the detector center along the beam pipe (z-axis).

\subsubsection{Electron Selection}

EM candidate objects have to satisfy the following requirements:

- $\mathrm{ID}^{1}=10$ or \pm 11 ;

- EM Fraction > 0.9;

- isolation $<0.15$;

- $\chi^{2}$ of H-Matrix (7) $<12$ if $\left|\eta_{\text {det }}\right|<1.1$;

- $\chi^{2}$ of H-Matrix $(7)<20$ if $1.5<\left|\eta_{\text {det }}\right|<2.5$;

- $p_{T}>20$;

- $\left|\eta_{\text {det }}\right|<2.5$ but not in $1.1<\left|\eta_{\text {det }}\right|<1.5$.

${ }^{1} \mathrm{EM}$ reconstruction algorithm assigns an ID of 10 for EM objects without a matched track. \pm 11 is an EM object with a matched track, and the sign is based on the curvature of the track. 


\subsubsection{Z Selection}

$Z$ candidates are selected based on the following criteria:

- two EM candidate objects;

- at least one electron is in the central calorimeter $\left|\eta_{\text {det }}\right|<1.1$;

- at least one electron with a track match $P\left(\chi^{2}\right)>0.01$ (using $\Delta \phi, \Delta \eta$, and $\left.E / p\right)$;

- one of the two electrons must have fired the trigger ${ }^{1}$;

- di-em invariant mass window cut $75 \mathrm{GeV}<\mathrm{M}_{e e}<105 \mathrm{GeV}$.

\subsubsection{Jets}

The Run II Midpoint Jet Cone Algorithm with a cone radius of 0.5 was used to identify jets. The following criteria were applied to select jet candidates:

- $0.05<$ EM fraction $<0.95$;

- coarse hadronic fraction $<0.4$;

- hot fraction $<10$;

- $\mathrm{n} 90>1$

- L1 confirmation > 0.4;

- $p_{T}>20 \mathrm{GeV}$ (after JES correction);

- $|\eta|<2.5$

\footnotetext{
${ }^{1}$ Matching trigger objects at L1, L2, and L3 within $\Delta R<0.4$ are required.
} 
- Since the jet algorithm forms a jet from the electron energy deposits, all jets overlapping with electrons identified as coming from the decay of the $Z / \gamma^{*}$ boson within $\Delta R=$ $\sqrt{(\Delta \eta)^{2}(\Delta \phi)^{2}}<0.5$ are removed.

\subsection{5 b-jet Selection}

b-jet candidate objects have to satisfy the following criteria:

- identified as taggable;

- identified as a b tag by JLIP.

\subsection{Data and MC Kinematic Comparisons of Electrons and Jets.}

Figure 47 shows the primary vertex for data and the PYтніA $Z \rightarrow e \bar{e}+X$ inclusive sample. Figure 48 through Figure 54 show comparisons of electron distributions for data and MC. The comparisons indicate that PYTHIA MC reasonably models the $Z$ inclusive data sample. 


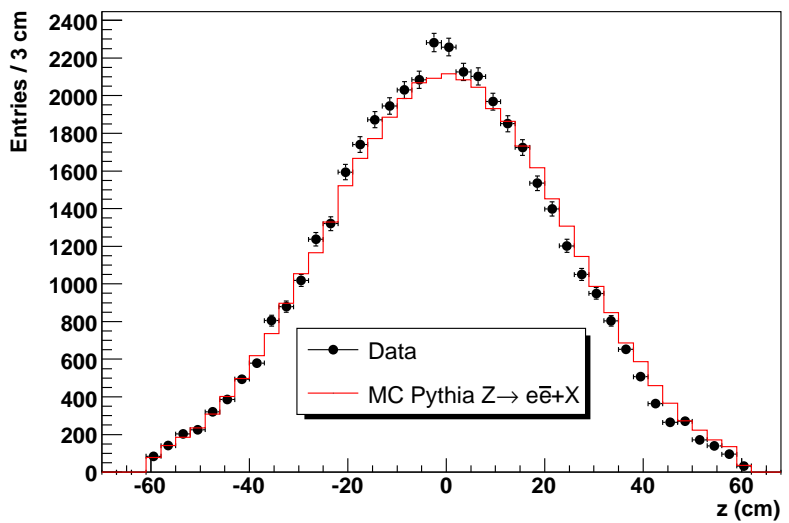

Figure 47. The primary vertex after the di-em invariant mass cut of $75 \mathrm{GeV}<M_{e e}<105$ $\mathrm{GeV}$ has been applied. The MC sample is normalized to the number of events in data.

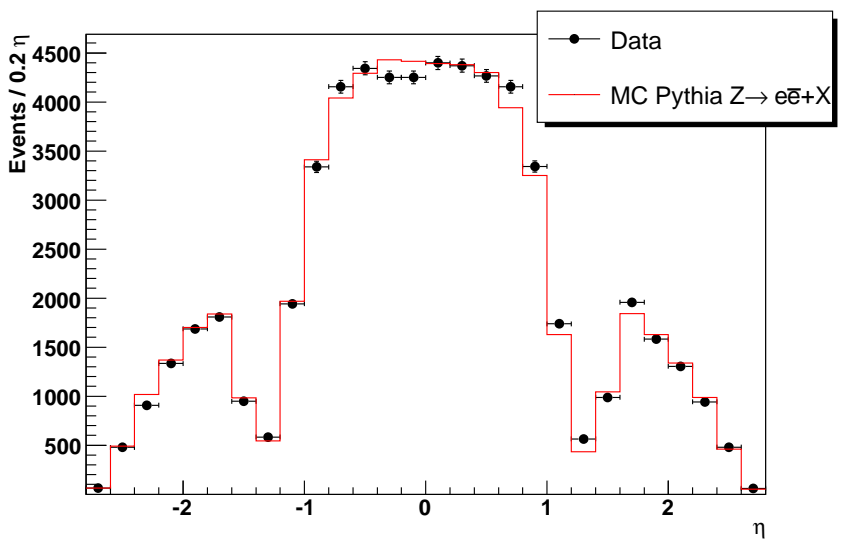

Figure 48. The $\eta$ distribution of the two electrons from the $Z$ boson after the di-em invariant mass cut of $75 \mathrm{GeV}<M_{e e}<105 \mathrm{GeV}$ has been applied. The MC sample is normalized to the number of events in data. 


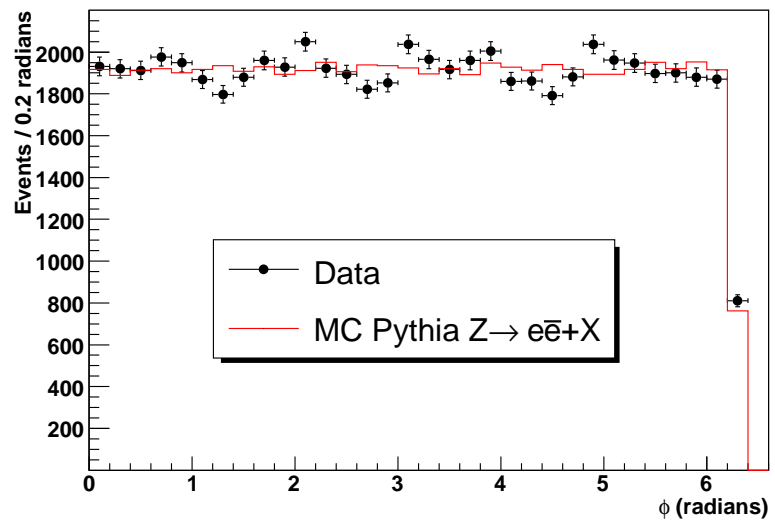

Figure 49. The $\phi$ distribution of the two electrons from the $Z$ boson after the di-em invariant mass cut of $75 \mathrm{GeV}<M_{e e}<105 \mathrm{GeV}$ has been applied. The MC sample is normalized to the number of events in data.

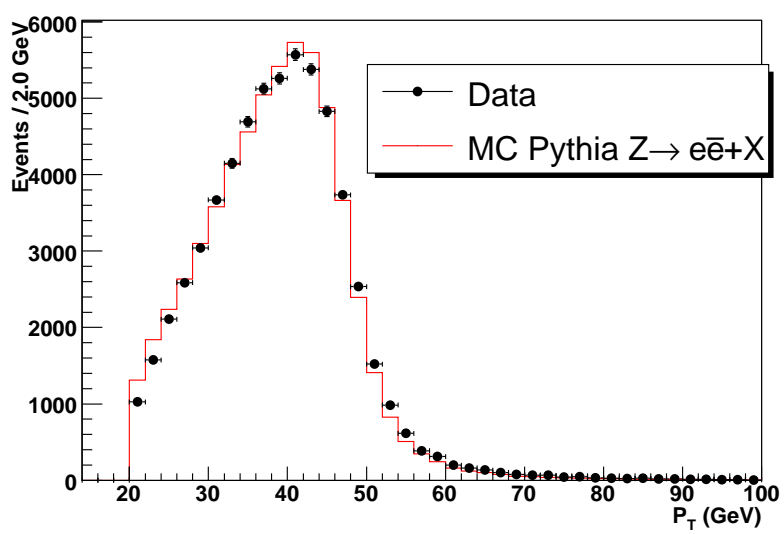

Figure 50. The $p_{T}$ distribution of the two electrons from the $Z$ boson after the di-em invariant mass cut of $75 \mathrm{GeV}<M_{e e}<105 \mathrm{GeV}$ has been applied. The MC sample is normalized to the number of events in data. 


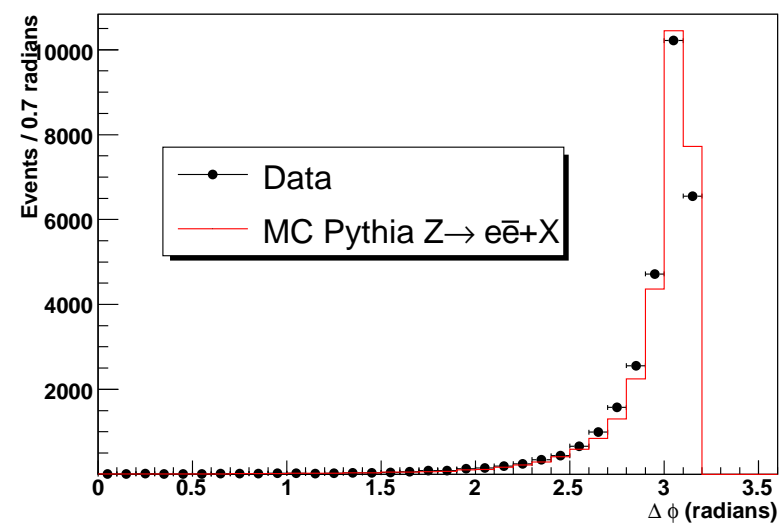

Figure 51. The $\Delta \phi$ distribution between the two electrons from the $Z$ boson after the di-em invariant mass cut of $75 \mathrm{GeV}<M_{e e}<105 \mathrm{GeV}$ has been applied. The MC sample is normalized to the number of events in data.

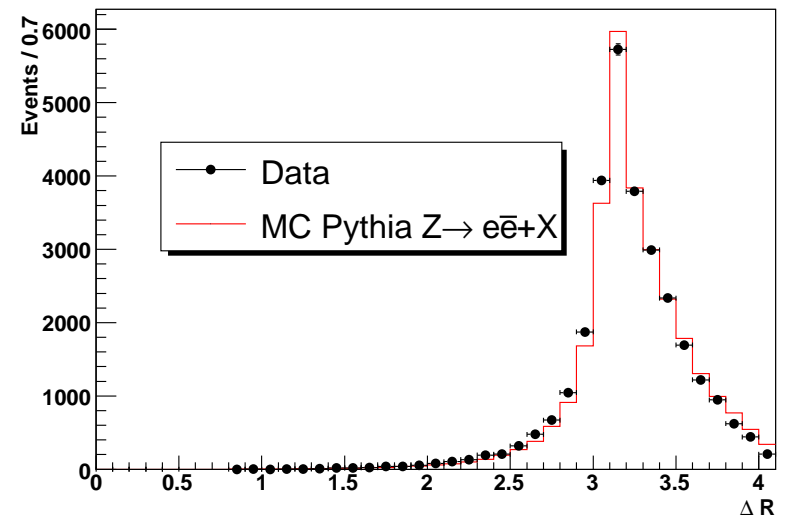

Figure 52. The $\Delta R$ distribution between the two electrons from the $Z$ boson after the di-em invariant mass cut of $75 \mathrm{GeV}<M_{e e}<105 \mathrm{GeV}$ has been applied. The MC sample is normalized to the number of events in data. 


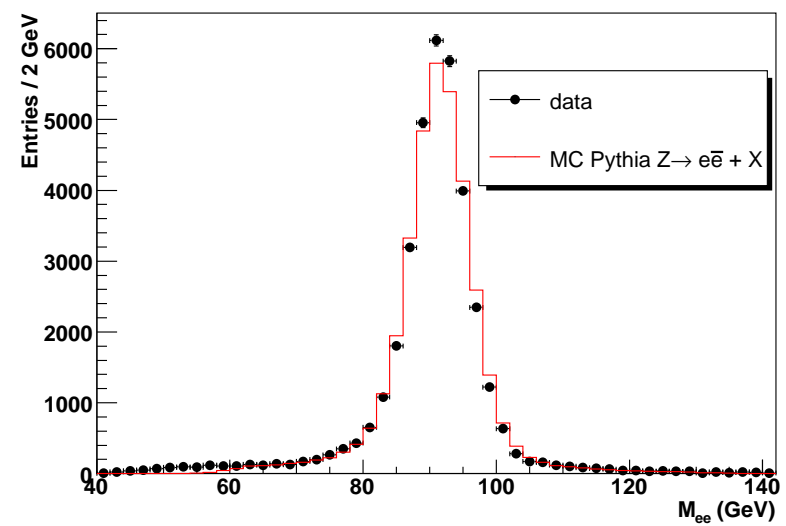

Figure 53. The di-em invariant mass distribution using two electron objects with track matches. PYTHIA is normalized to have the same number of events in the window of $80 \mathrm{GeV}$ $<\mathrm{M}_{e e}<100 \mathrm{GeV}$ as data.

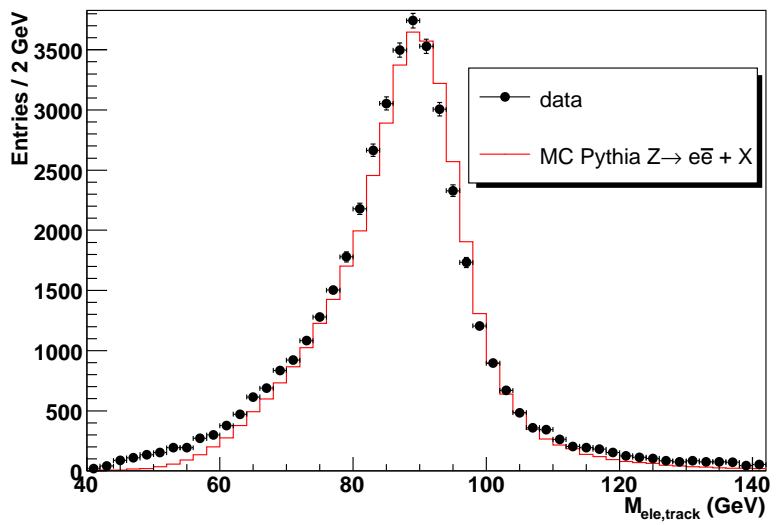

Figure 54. The di-em invariant mass distribution using an electron with a track match and an opposite sign track. The momentum vectors of the opposite sign tracks in the MC are smeared to have the same momentum resolution as the probe tracks in data. PYTHIA is normalized to have the same number of events in the window of $75 \mathrm{GeV}<\mathrm{M}_{e e}<105 \mathrm{GeV}$ as data. 
Figure 55 shows the $Z$ candidate $p_{T}$ distribution for data and PYTHIA $Z / \gamma^{*} \rightarrow e^{+} e^{-}+X$ MC. Figure 56 through Figure 58 show jet comparison distributions for the same MC sample. PYTHIA MC models the data jet distributions reasonably well.

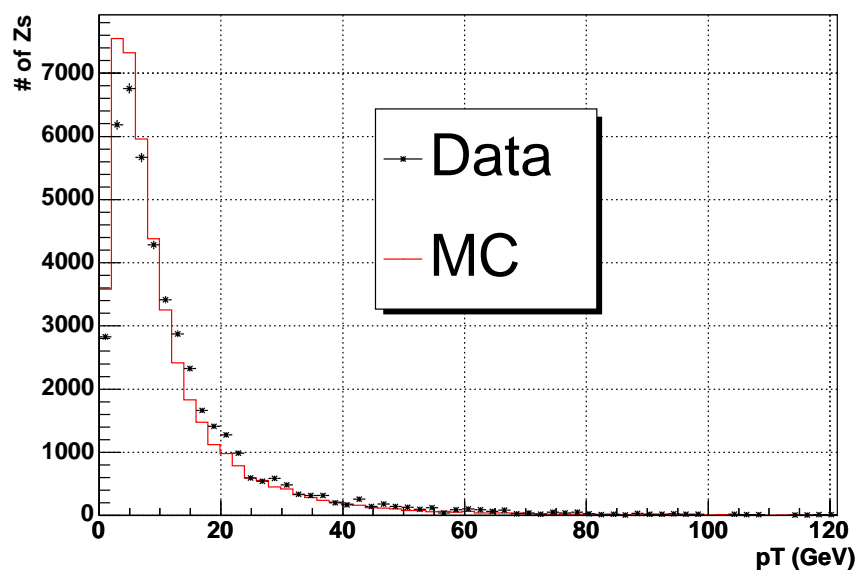

Figure 55. $Z p_{T}$ distribution for the $Z$ inclusive sample after the di-em invariant mass cut of $75 \mathrm{GeV}<M_{e e}<105 \mathrm{GeV}$ has been applied. The PythiA MC sample is normalized to the number of events in data. 


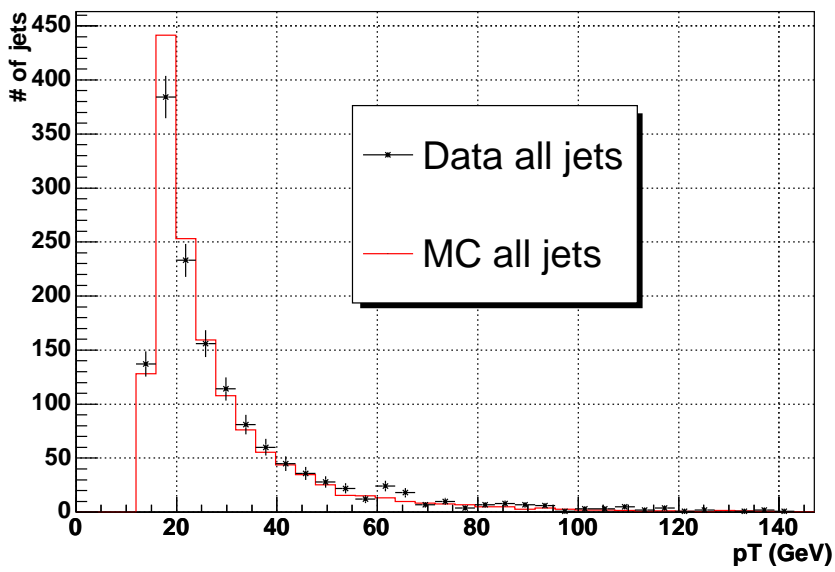

Figure 56. Jet $p_{T}$ distribution in the $Z$ inclusive sample after the di-em invariant mass cut of $75 \mathrm{GeV}<M_{e e}<105 \mathrm{GeV}$ has been applied. The PYTHIA MC sample is normalized to the number of events in data.

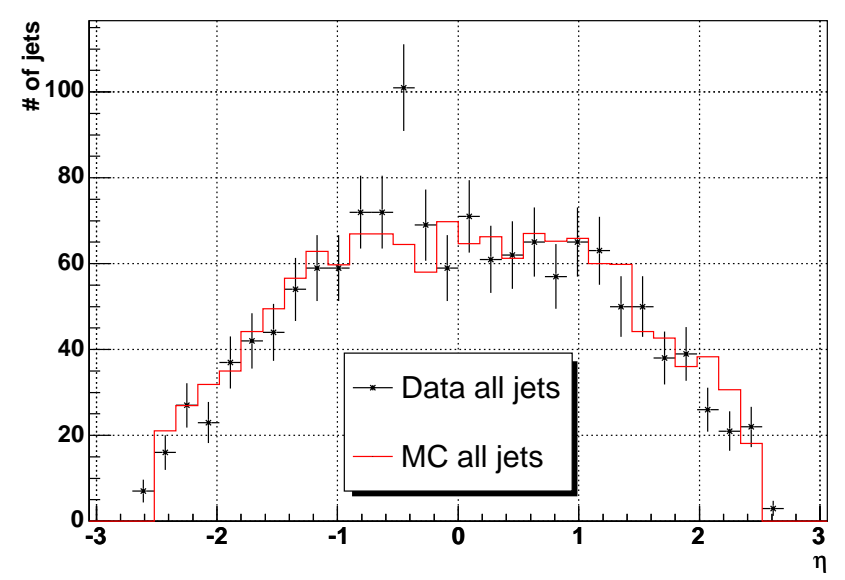

Figure 57. Jet $\eta$ distribution for the $Z$ inclusive sample after the di-em invariant mass cut of $75 \mathrm{GeV}<M_{e e}<105 \mathrm{GeV}$ has been applied. The Pythia MC sample is normalized to the number of events in data. 


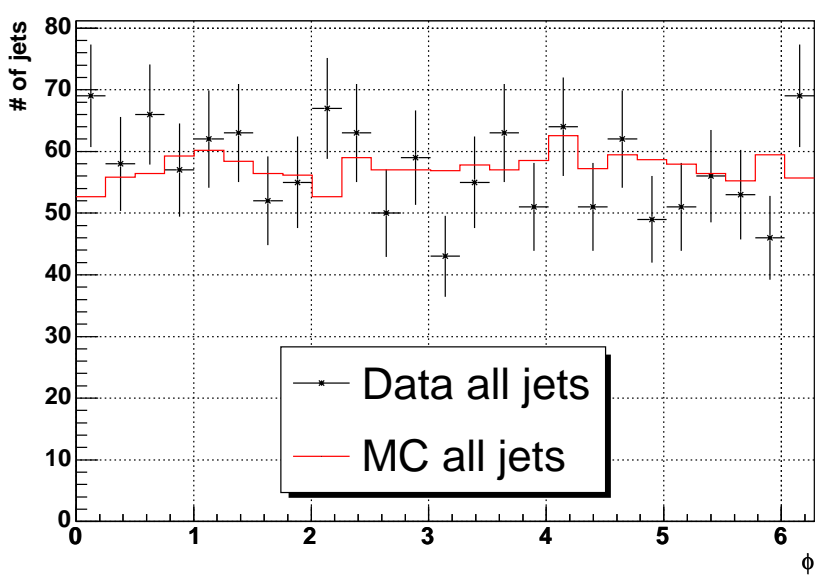

Figure 58. Jet $\phi$ distribution for the $Z$ inclusive sample after the di-em invariant mass cut of $75 \mathrm{GeV}<M_{e e}<105 \mathrm{GeV}$ has been applied. The Pythia MC sample is normalized to the number of events in data. 
Figure 59 through Figure 61 show comparisons between data and the ALPGEN + PYTHIA MC Zjj sample for taggable jets. The agreement is satisfactory.

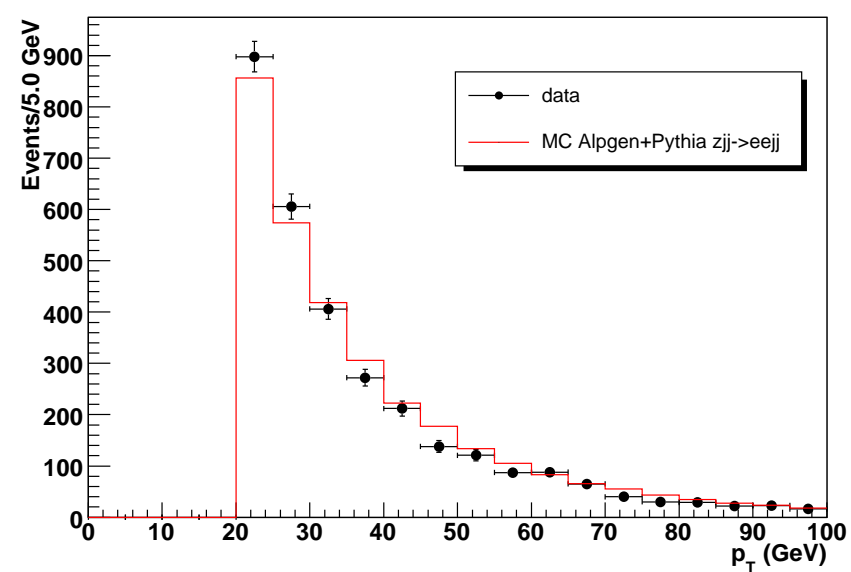

Figure 59. Taggable jet $p_{T}$ distributions for events that have a $\mathrm{Z}$ boson and at least one calorimeter jet. The ALPGEN+PYTHIA sample is normalized to the number of events in data. 


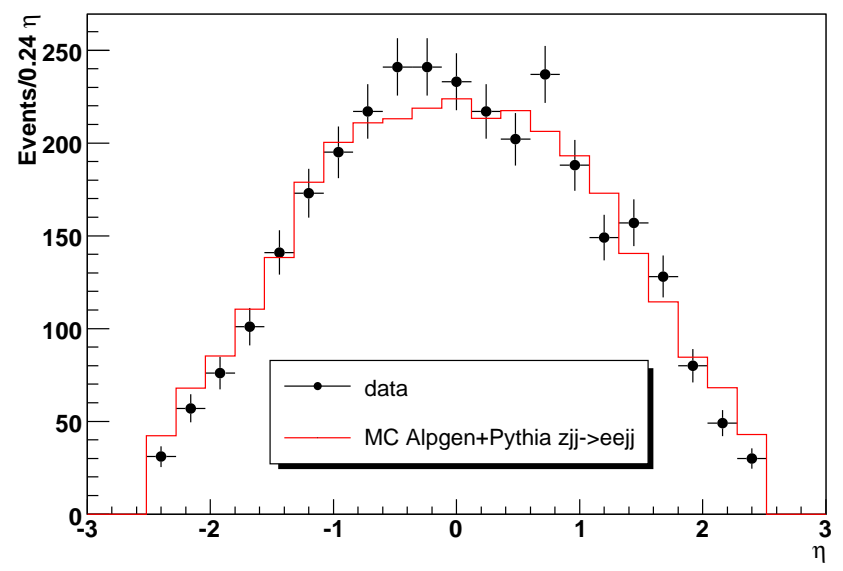

Figure 60. Taggable jet $\eta$ distributions for events that have a $\mathrm{Z}$ boson and at least one calorimeter jet. The ALPGEN+PYTHIA sample is normalized to the number of events in data.

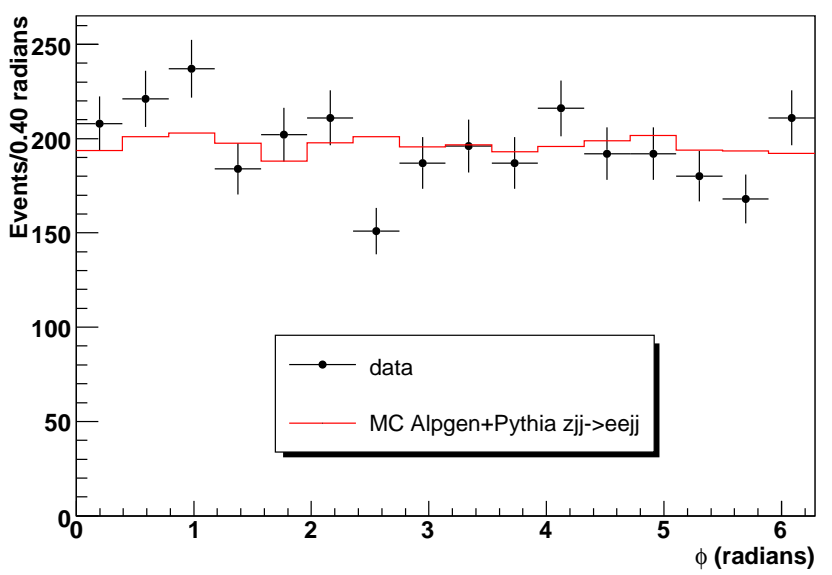

Figure 61. Taggable jet $\phi$ distributions for events that have a $\mathrm{Z}$ boson and at least one calorimeter jet. The ALPGEN+PYTHIA sample is normalized to the number of events in data. 


\section{CHAPTER 8}

\section{OBJECT RECONSTRUCTION AND SELECTION EFFICIENCIES}

This chapter describes the efficiency measurements of the object reconstruction and selection. The efficiencies are measured separately in data and MC. The efficiencies are parameterized with two dimensional fits which take into account the correlations of the two variables. The first requirement of this analysis is to have two EM objects and two jets in the events. Calculating the efficiencies (trigger, EM object, track match) in a data sample with a $Z$ boson candidate and two high- $p_{T}$ jets dramatically decreases the statistics of the sample and makes 2-dimensional (2D) parameterizations difficult. Therefore, the parameterizations are done on an inclusive $Z$ sample with zero or more jets. Overall object reconstruction and selection efficiencies are examined versus jet multiplicity. Differences between the average inclusive and $\geq 2$ jets efficiency measurements are included as a systematic uncertainty.

The parameterization of the selection cut efficiencies per object is used to tune the MC to match the selection efficiencies in data. The tuning of the MC for a given efficiency is performed by applying a multiplicative scale factor, eff ${ }_{d a t a} /$ eff $_{M C}$, to each object. A random number is generated for each MC object and if the random number is greater than the scale factor value, the object is dropped from the event. This chapter outlines the efficiency calculations for the trigger selection, reconstruction and identification of EM objects, EM object track matching, reconstruction and identification of jet objects, and taggable and b-tagged jet requirements. 


\subsection{Trigger Efficiency}

The events in data have acceptance losses caused by the trigger selection requirements. These losses are incorporated in the MC simulation by applying the trigger efficiencies measured in data. The trigger efficiency is studied using the trigger mapping scheme as shown in Table VIII from Chapter 7. A reconstructed electron candidate is required to pass the conditions of all three trigger levels. The electron satisfies the L1 requirement if there is a trigger

tower within a distance of $\Delta R=\sqrt{(\Delta \phi)^{2}+(\Delta \eta)^{2}}<0.4$. The trigger tower has to have enough electromagnetic energy to pass the L1 threshold. Similarly, L2 and L3 trigger objects need to be above the trigger threshold and within $\Delta R<0.4$ of the reconstructed electron.

The trigger efficiency per electron is studied with a tag-and-probe method using $Z$ boson candidate events with an di-electron invariant mass between 70 and $110 \mathrm{GeV}$. In this method, both $Z$ candidate electrons are considered as possible "tags". The "tag" electron is required to pass all the trigger conditions, at each trigger level, that fired the event. Both tag and probe electrons must satisfy the following requirements:

- $p_{T}>20 \mathrm{GeV}$;

- EM Fraction > 0.9;

- Isolation $<0.15$

- $\chi^{2}$ of H-Matrix $(7)<12$ if $\left|\eta_{\text {det }}\right|<1.1$;

- $\chi^{2}$ of H-Matrix $(7)<20$ if $1.5<\left|\eta_{\text {det }}\right|<2.5$;

- Track match with $P\left(\chi^{2}\right)>0.01$ (using $\Delta \phi, \Delta \eta$, and $E / p$ ). 
The trigger efficiency is measured as a function of $p_{T}$ and $\eta_{\text {det }}$ of the probe electron. An efficient probe electron must have a matching L1, L2, and L3 trigger objects within $\Delta R<$ 0.4. Figure 62 through Figure 64 show the trigger efficiencies as a function of $p_{T}, \eta_{d e t}$, and $\phi$. The average electron trigger efficiencies for various inclusive jet multiplicities, after background subtraction, are shown in Table XIII. The trigger efficiency parameterization is done using a "combined" (using v8 through v13) trigger list sample, which properly weights the effect of each version of the trigger with respect to the total data sample. The combined trigger efficiency has an average value of $95 \%$ per $Z$ boson electron. The trigger efficiencies are also examined as a function of jet multiplicity, summarized in Table XIII, and shown in Figure 65 and Figure 66. Since the data sample recorded with v8 to v10 only makes up about $60 \mathrm{pb}^{-1}$ of the $452 \mathrm{pb}^{-1}$ of collected data, the $4 \%$ drop in efficiency for the $Z+\geq 2 j$ sample observed for the running period has a negligible overall contribution. The single-object trigger efficiency for $Z$ total inclusive sample (i.e. $Z+\geq 0 j$ ) falls within the statistical uncertainty of the efficiency for the $Z+\geq 2 j$ sample (Figure 66. Since there are two electrons coming from the $Z$ boson, the event trigger efficiency is evaluated by Equation 8.1 yielding an overall event trigger efficiency of $\sim 100 \%$.

$$
\epsilon_{\text {trigger }}=\epsilon_{1} \cdot\left(1-\epsilon_{2}\right)+\epsilon_{2} \cdot\left(1-\epsilon_{1}\right)+\epsilon_{1} \cdot \epsilon_{2}
$$

The statistical uncertainty of the object based trigger efficiency is estimated to be $0.11 \%$ (Table XIII). A systematic uncertainty of $0.2 \%$ for the electron trigger efficiency is assigned based on the efficiency change when the track match requirement on the probe electron object is removed. A systematic uncertainty of $1.1 \%$ for the electron trigger efficiency is assigned based 
on the difference of the overall efficiency between the $Z$ inclusive sample and the $Z+\geq 2 j$ sample. The three uncertainties added in quadrature give an overall uncertainty of $1.2 \%$ for the trigger efficiency for the $Z+\geq 2 j$ sample.

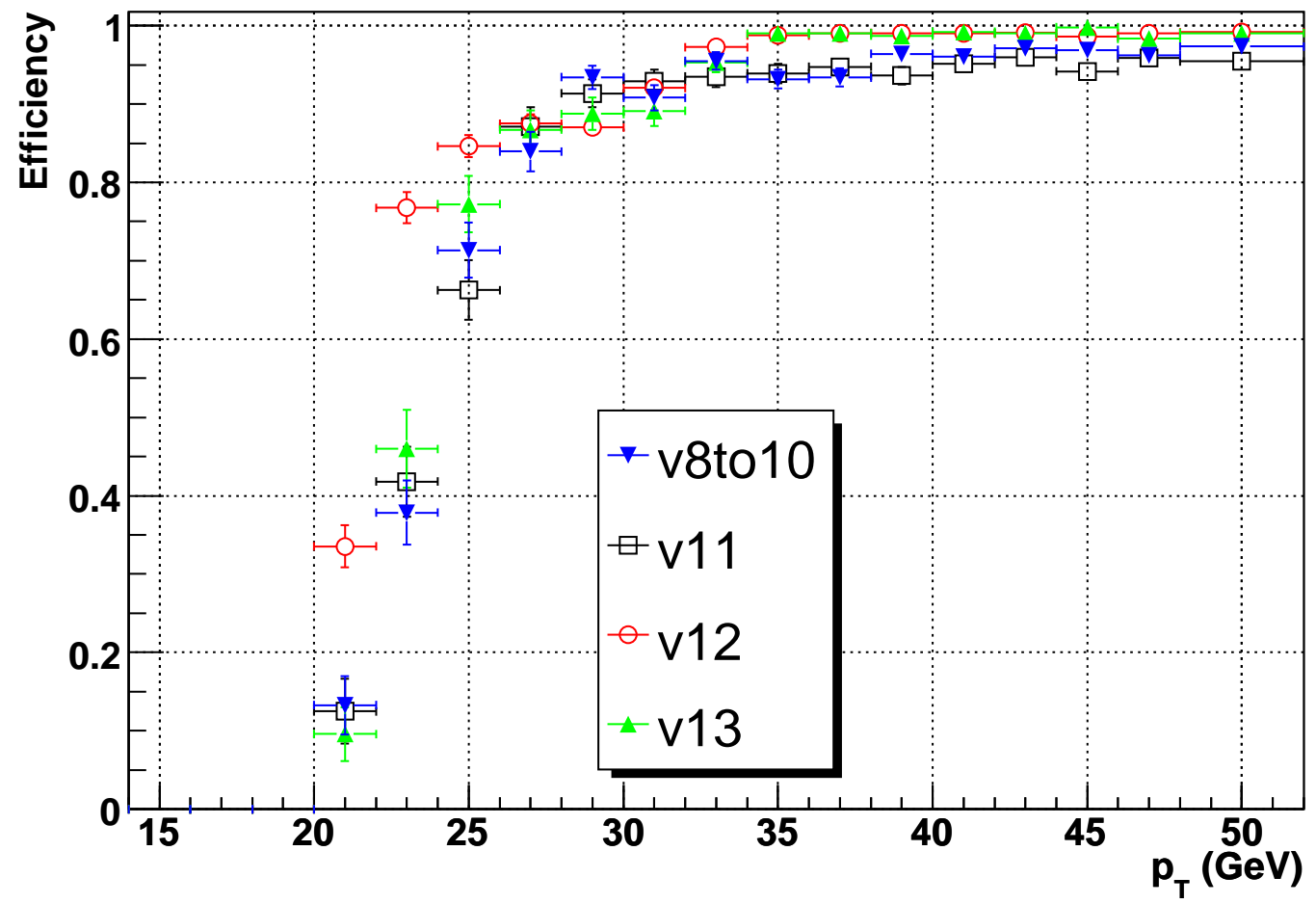

Figure 62. Trigger efficiencies vs electron $p_{T}$ with zero or more jets in each event for different trigger list versions. 


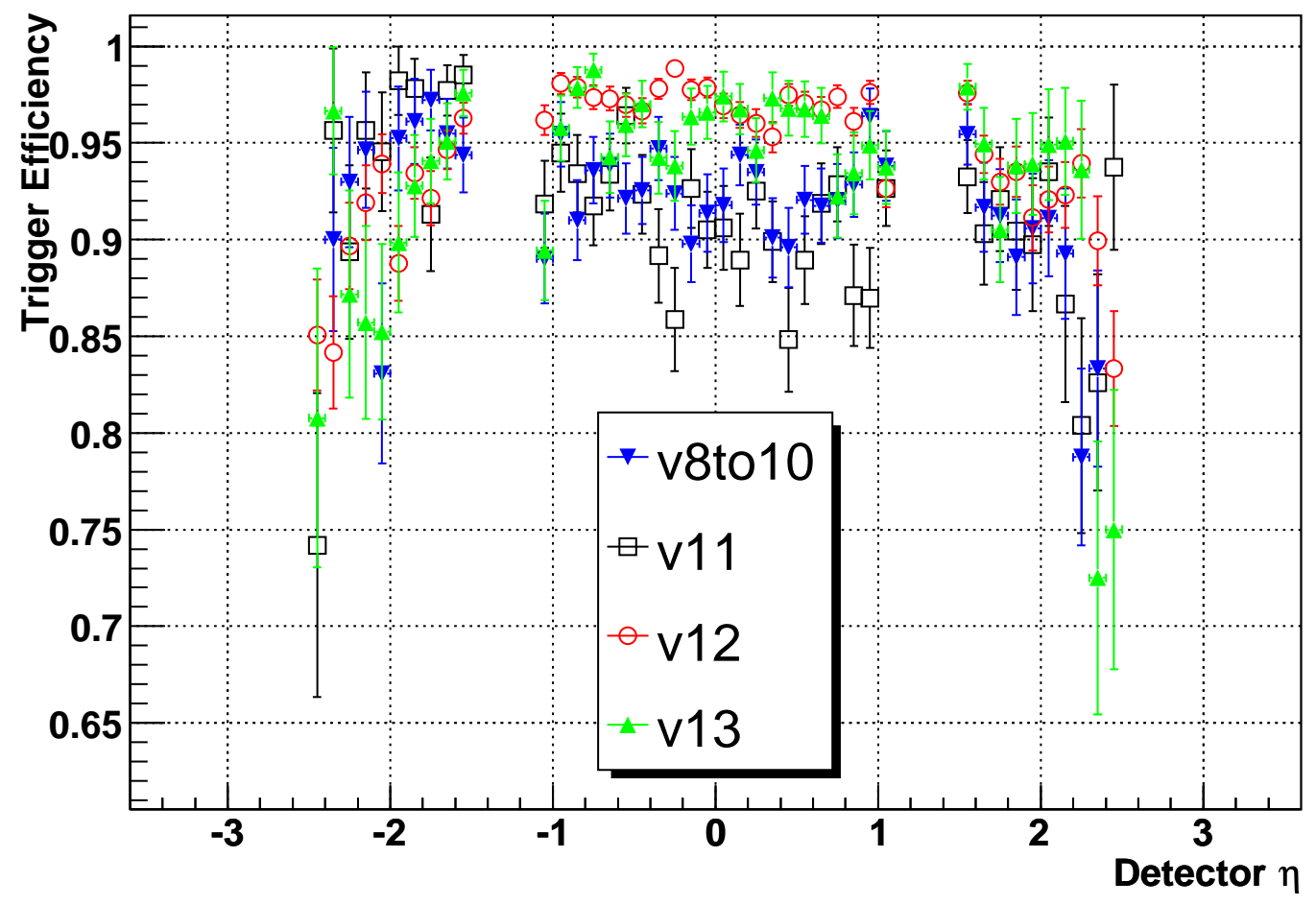

Figure 63. Trigger efficiencies vs electron $\eta_{\text {det }}$ with zero or more jets in each event for different trigger list versions. 


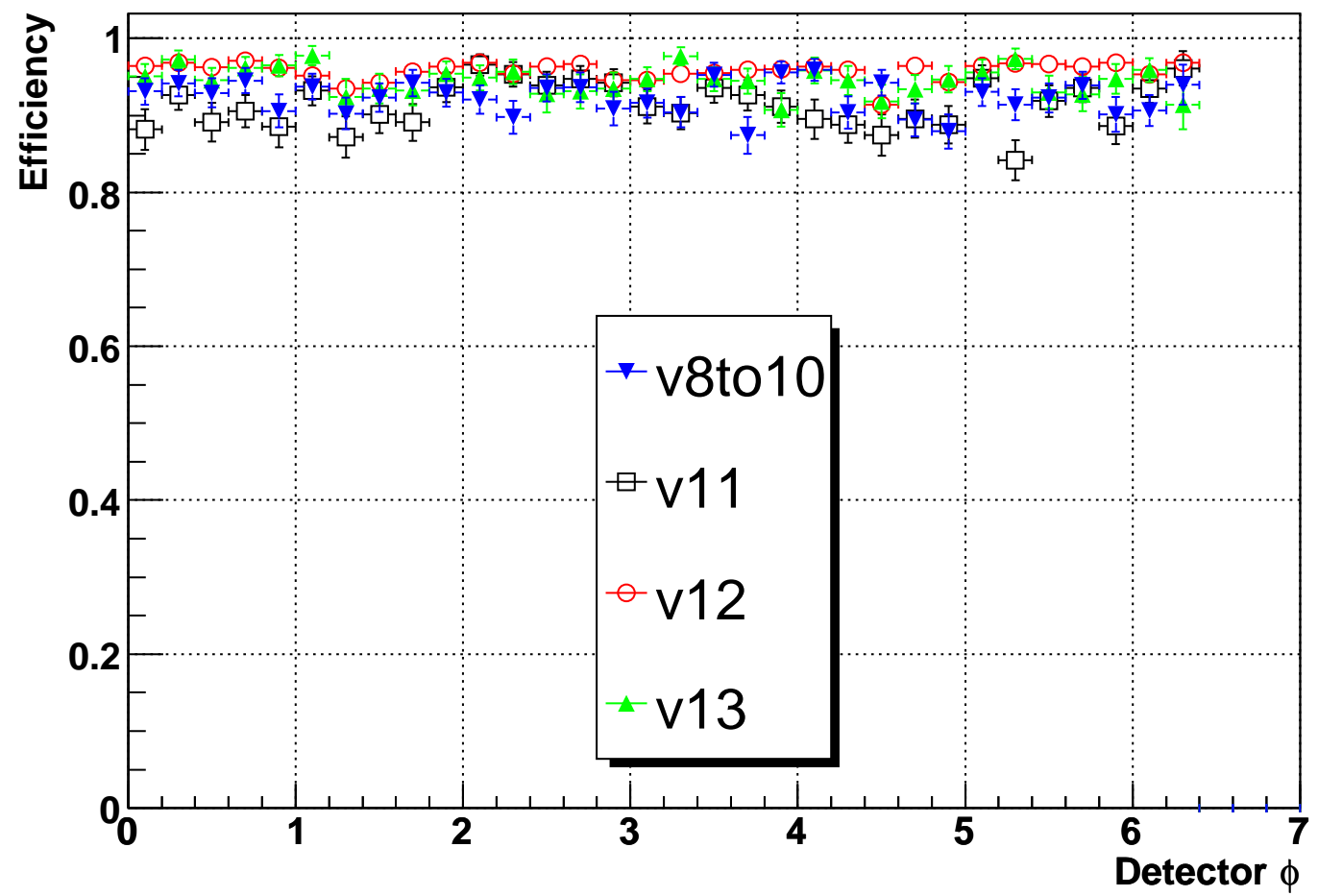

Figure 64. Trigger efficiencies vs electron $\phi_{\text {det }}$ with zero or more jets in each event for different trigger list versions. 


\begin{tabular}{|c|c|c|c|}
\hline Trigger Version & $>=0$ jets & $>=1$ jet & $>=2$ jets \\
\hline v8to10 & $92.2 \pm 0.3$ & $90.7 \pm 1.2$ & $88 \pm 4$ \\
v11 & $91.3 \pm 0.4$ & $89.6 \pm 1.3$ & $94 \pm 3$ \\
v12 & $95.8 \pm 0.1$ & $95.2 \pm 0.4$ & $97 \pm 1$ \\
v13 & $94.6 \pm 0.3$ & $93.6 \pm 1.0$ & $98 \pm 2$ \\
Combined & $95.0 \pm 0.1$ & $93.4 \pm 0.4$ & $95 \pm 1$ \\
\hline
\end{tabular}

TABLE XIII

AVERAGE TRIGGER EFFICIENCIES (\%) WITH RESPECT TO TRIGGER VERSION AND JET MULTIPLICITY. THE UNCERTAINTIES GIVEN ARE STATISTICAL. 


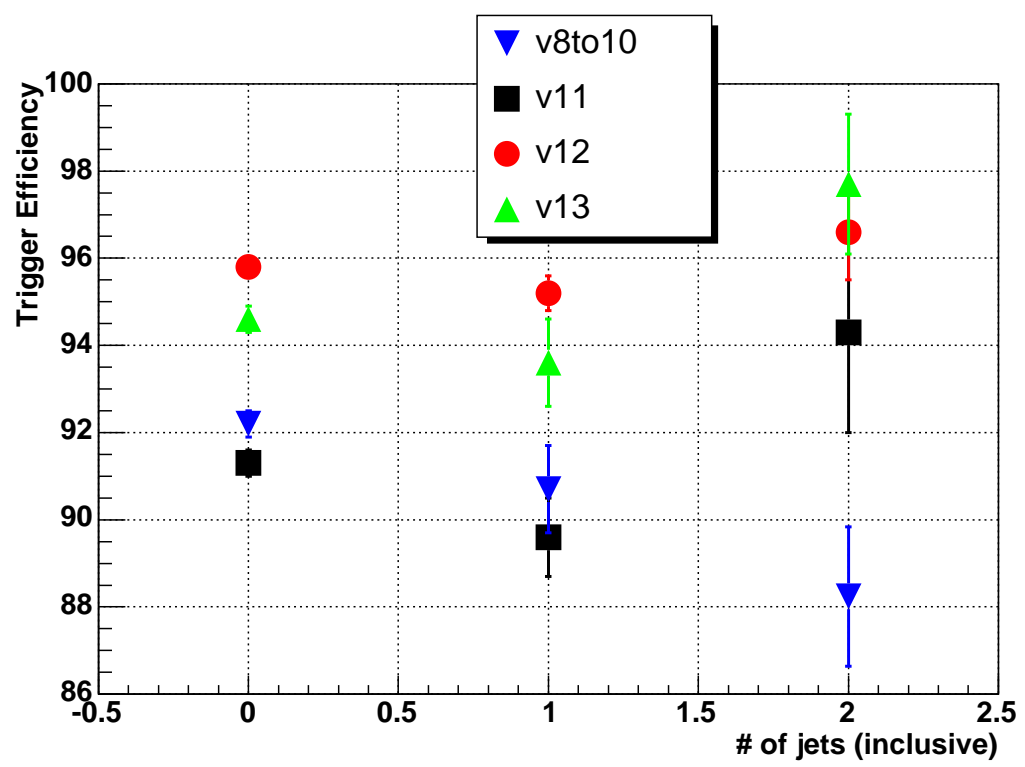

Figure 65. Trigger efficiencies as a function of jet multiplicity.

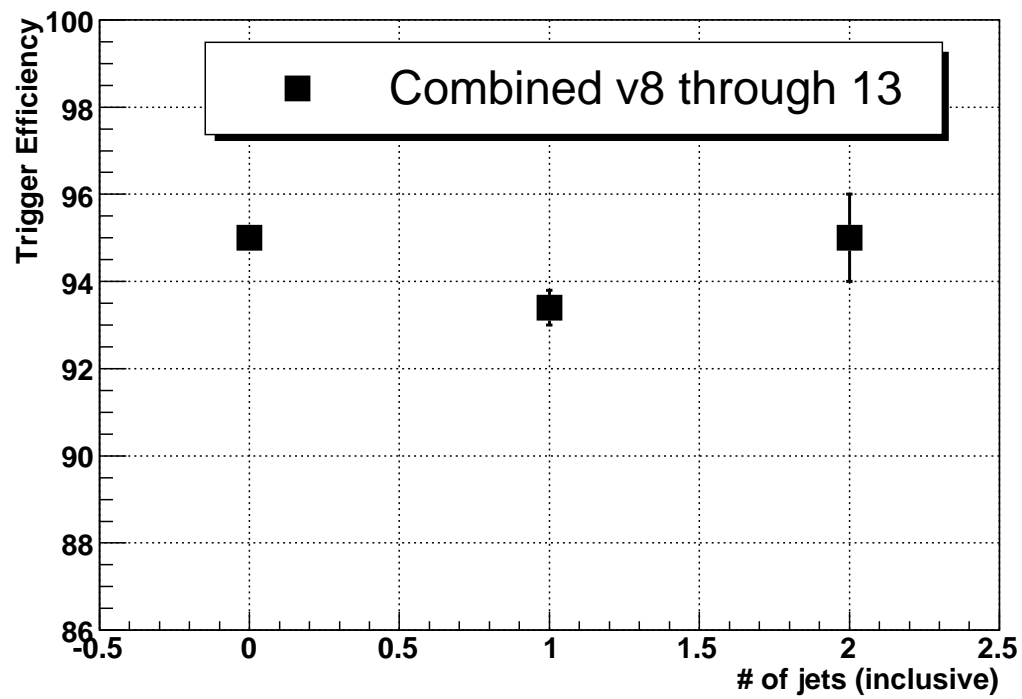

Figure 66. Trigger efficiencies as a function of jet multiplicity for the combined data sample. 


\subsection{Electron Reconstruction and Identification Efficiency}

The reconstruction and identification (RECO/ID) efficiencies of the EM objects are determined for various jet multiplicities in data and MC. The efficiencies are measured using the electrons from $Z$ boson decays. In the central region of the calorimeter $\left|\eta_{\text {det }}\right|<1.1$, the efficiencies are studied versus the $p_{T}$ and the $\phi+2 \pi$ floating point remainder of $2 \pi / 32$ of the probe track. As discussed in 3.2.5, there are cracks in between $\phi$ modules every 0.196 radians causing efficiency losses. The floating point remainder will range from 0 to 0.2 , so that all 32 calorimeter modules can be overlaid into one histogram. In the forward regions of the calorimeter 1.5 $<\left|\eta_{\text {det }}\right|<2.5$, the efficiencies are determined versus the $p_{T}$ and $\eta_{\text {det }}$ of the probe track. The efficiencies are calculated using a tag and probe method. The tag electron must satisfy the following conditions:

- $p_{T}>20 \mathrm{GeV}$

- EM Fraction > 0.95;

- Isolation $<0.10$

- $\chi^{2}$ H-Matrix $(7)<9$ if $\left|\eta_{\text {det }}\right|<1.1$;

- $\chi^{2} \mathrm{H}-\operatorname{Matrix}(7)<17$ if $1.5<\left|\eta_{\text {det }}\right|<2.5$;

- Passes the trigger (data only) with $\Delta R<0.4$ of L1, L2, and L3 trigger objects;

- Spatial track match with $\Delta R<0.14$;

- Track isolation $\left(\Sigma p_{T}\right.$ of tracks within $\left.\Delta R<0.3\right)<0.25$ probe track $p_{T}$.

The track requirements for the tag and probe are: 
- Stereo track (requires hits in u v stereo layers);

- $20<p_{T}<160 \mathrm{GeV}$;

- $\chi^{2}$ probability for the best track $<8.0$;

- Distance of closest approach between the track and beam position in the $R-\phi$ plane $<$ $0.3 \mathrm{~cm}$;

- Distance of closest approach between the track and beam position along the $\mathrm{Z}$ axis $<4.0$ $\mathrm{cm}$

- Track isolation $\left(\Sigma p_{T}\right.$ of tracks with $\left.\Delta R<0.3\right)<0.25$ probe track $p_{T}$.

The probe is a track which has to fulfill the track requirements and to have the opposite sign of the tag track. The probe track scans for a reconstructed electron within $\Delta R<0.14$, that passes the cuts of EM fraction, isolation, and $\chi^{2}$ of H-matrix 7 cuts.

The electron matching requirements for an efficient EM object are:

- $\mathrm{ID}=10$ or \pm 11 ;

- EM Fraction > 0.9;

- Isolation $<0.15$

- $\chi^{2}$ of $\mathrm{H}-\operatorname{Matrix}(7)<12$ if $\left|\eta_{\text {det }}\right|<1.1$;

- $\chi^{2}$ of H-Matrix $(7)<20$ if $1.5<\left|\eta_{\text {det }}\right|<2.5$;

- Within $\Delta R<0.14$ of the probe track. 
The invariant mass derived from the tag electron and the probe track have to be within $80 \mathrm{GeV}<M_{\text {tag }_{\text {electron }} \text { probe }_{\text {track }}}<100 \mathrm{GeV}$. The sidebands of the invariant mass peak (starting $10 \mathrm{GeV}$ away from the mass window) are used to estimate the background contribution from QCD process to the average efficiency. A $2 \mathrm{D}\left(p_{T}, \phi+2 \pi\right.$ floating point remainder of $\left.2 \pi / 32\right)$ parameterization is made for tracks with $\left|\eta_{\text {det }}\right|<1.1$ and another $2 \mathrm{D}\left(p_{T}, \eta_{\text {det }}\right)$ parameterization is made for forward tracks. Figure 67 through Figure 70 show the efficiencies in $1 \mathrm{D}$ plots measured in data and MC. The efficiencies are also examined as a function of jet multiplicity as shown in Table XIV and Figure 71.

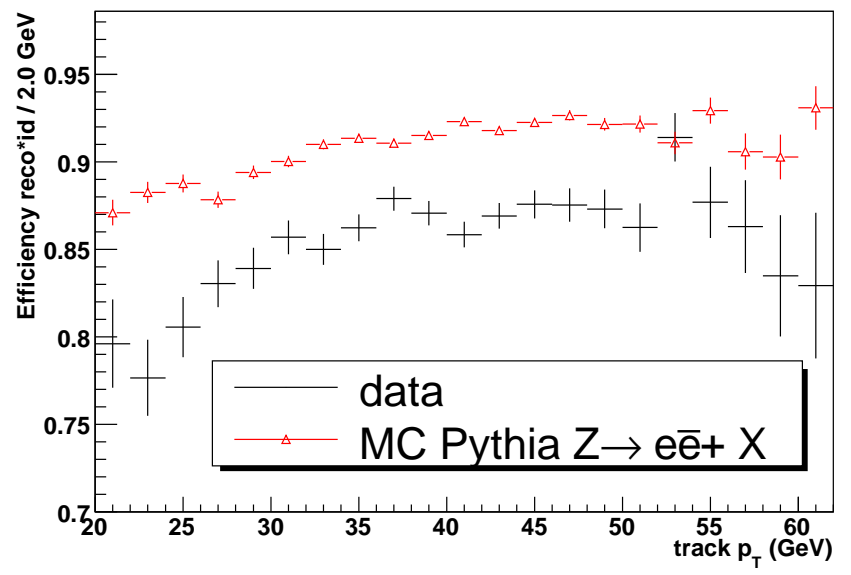

Figure 67. Electron RECO/ID efficiencies in data and MC from events using the $Z$ inclusive sample vs probe track $p_{T}$ without background subtraction. The MC sample is PYTHIA generated $Z \rightarrow e \bar{e}+X$. The probe track is in the central region $\left|\eta_{\text {det }}\right|<1.1$ of the calorimeter. 


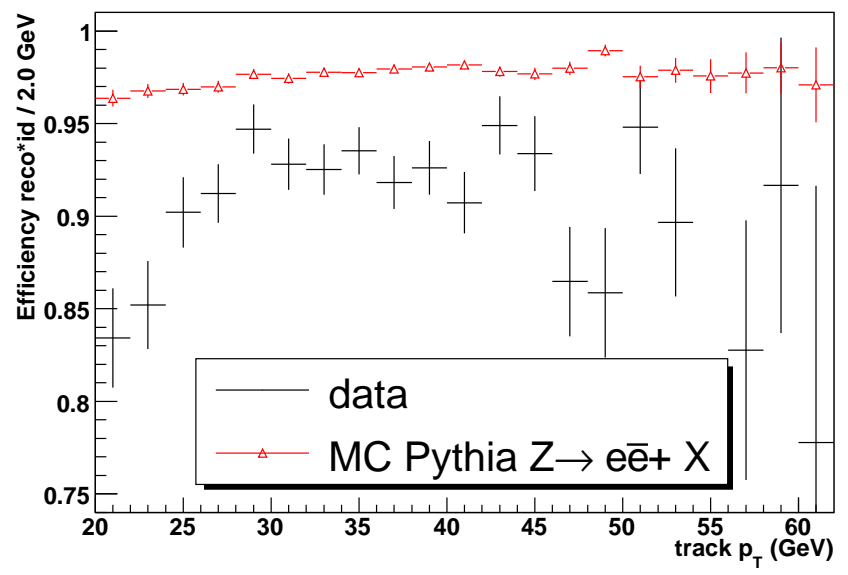

Figure 68. Electron RECO/ID efficiencies in data and $\mathrm{MC}$ from events using the $Z$ inclusive sample vs probe track $p_{T}$ without background subtraction. The MC sample is PYTHIA generated $Z \rightarrow e \bar{e}+X$. The probe track is in the forward region $1.5<\left|\eta_{\text {det }}\right|<2.5$ of the calorimeter.

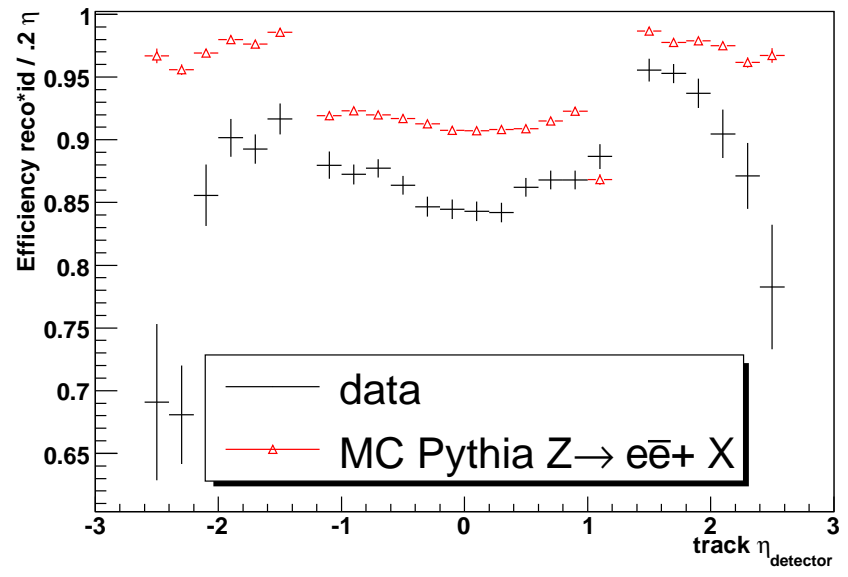

Figure 69. Electron RECO/ID efficiencies in data and $\mathrm{MC}$ from events using the $Z$ inclusive sample vs probe track $\eta_{\text {det }}$ without background subtraction. The MC sample is PYTHIA generated $Z \rightarrow e \bar{e}+X$. 


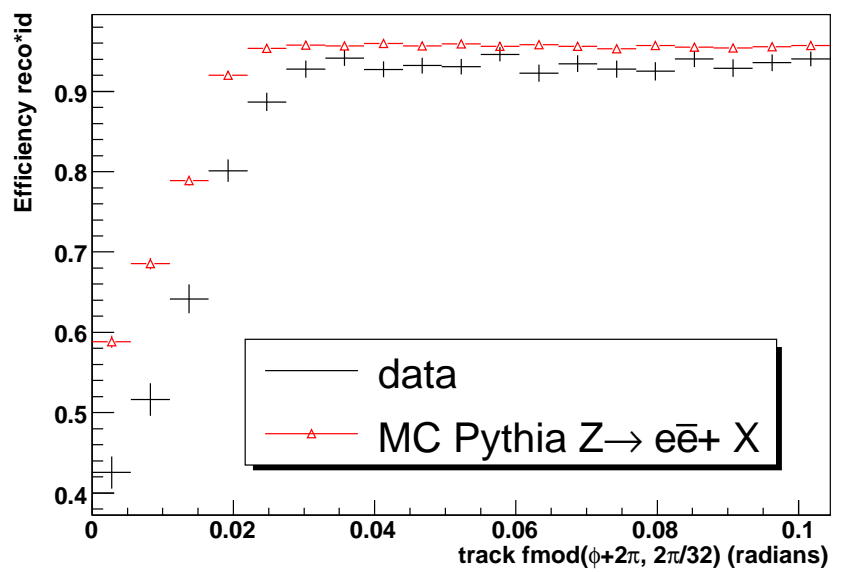

Figure 70. Electron RECO/ID efficiencies in data and MC from events using the $Z$ inclusive sample vs probe track $\phi+2 \pi$ floating point remainder of $2 \pi / 32$ without background subtraction. The MC sample is PYTHIA generated $Z \rightarrow e \bar{e}+X$. 


\begin{tabular}{|c|c|c|c|}
\hline Sample & $>=0$ jets & $>=1$ jet & $>=2$ jets \\
\hline Data & $88.9 \pm 0.2$ & $88.1 \pm 0.7$ & $90.5 \pm 1.8$ \\
Monte Carlo & $92.7 \pm 0.06$ & $91.9 \pm 0.2$ & $91.7 \pm 0.6$ \\
\hline
\end{tabular}

TABLE XIV

\section{BACKGROUND SUBTRACTED ELECTRON RECO/ID EFFICIENCIES VS JET MULTIPLICITY FOR DATA AND MC. THE UNCERTAINTIES ARE STATISTICAL.}

The uncertainty for the electron RECO/ID efficiencies was estimated from four sources. The first source is from the statistical uncertainty of the average efficiency value for $Z+\geq 0 j$, $0.2 \%$. The second source is a systematic uncertainty coming from the difference between the $Z+\geq 0 j$ average efficiency value and the $Z+\geq 2 j$ average efficiency value in data: $1.8 \%$. The third source is estimated from a systematic uncertainty due to the dependence of the di-em invariant mass window used for background subtraction: $0.8 \%$. The fourth source is a systematic uncertainty due to the difference between the $Z+\geq 0$ average efficiency and the $Z+\geq 2$ average efficiency in MC, which will only add a positive contribution to the uncertainty: $1.1 \%$. The first three sources of uncertainty are added in quadrature with the fourth source added in linearly. The overall EM RECO/ID efficiency uncertainty is $\pm 2.0_{-0}^{+1.1} \%$ and summarized in Table XV. 


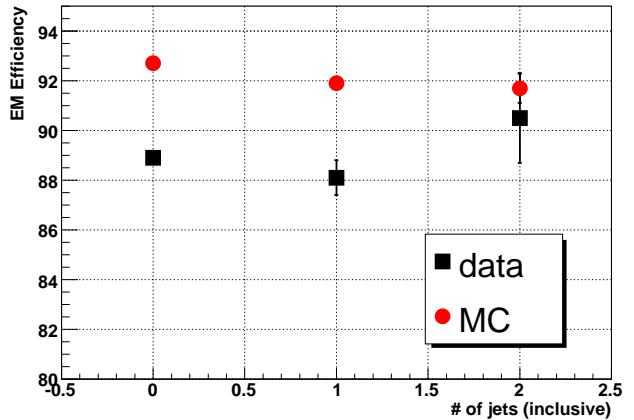

Figure 71. Electron RECO/ID efficiency vs jet multiplicity for data and MC after background subtraction. The sidebands of the invariant mass peak are used for the background estimation.

\begin{tabular}{|c|c|}
\hline Uncertainty due to & $\%$ \\
\hline Statistical & 0.2 \\
Avg eff data $Z+\geq 0 j$ - Avg eff data $Z+\geq 2 j$ & 1.8 \\
Background subtraction di-em invariant mass window & 0.8 \\
Avg eff MC $Z+\geq 0 j$ jet - Avg eff $\mathrm{MC} Z+\geq 2 j$ & +1.1 \\
\hline Overall & $\pm 2.0_{-0}^{+1.1}$ \\
\hline
\end{tabular}

TABLE XV

ELECTRON RECO/ID UNCERTAINTIES. 


\subsection{Electron-Track Match Efficiency}

The track finding and matching efficiencies are measured in data and MC using electrons from $Z$ boson decays. In the central region of the calorimeter $\left|\eta_{\text {det }}\right|<1.1$, the efficiencies are found to be dependent on the electron $p_{T}$. In the forward region, $1.5<\left|\eta_{\text {det }}\right|<2.5$, the efficiencies are found to be dependent on electron $p_{T}$ and $\eta_{\text {det }}$. The efficiencies are calculated using a tag and probe method.

The tag electron must satisfy the following conditions:

- $p_{T}>20 \mathrm{GeV}$;

- EM Fraction > 0.9;

- Isolation $<0.15$

- $\chi^{2}$ H-Matrix $(7)<12$ if $\left|\eta_{\text {det }}\right|<1.1$;

- $\chi^{2} \mathrm{H}-\operatorname{Matrix}(7)<20$ if $1.5<\left|\eta_{\text {det }}\right|<2.5$;

- Passes the trigger (data only) with $\Delta R<0.4$ of L1, L2, and L3 trigger objects;

- Spatial track match with $\Delta R<0.14$.

The probe electron must satisfy the following conditions:

- $p_{T}>20 \mathrm{GeV}$;

- EM Fraction > 0.9;

- Isolation $<0.15$

- $\chi^{2}$ H-Matrix(7) $<12$ if $\left|\eta_{\text {det }}\right|<1.1$; 
- $\chi^{2}$ H-Matrix $(7)<20$ if $1.5<\left|\eta_{\text {det }}\right|<2.5$.

The tag and the probe electrons have to be within the di-em invariant mass window of $75 \mathrm{GeV}$ $<M_{e e}<105 \mathrm{GeV}$. A probe electron has a track match if the track match probability (using $\Delta \phi, \Delta \eta$, and $\mathrm{E} / \mathrm{p}), \mathrm{P}\left(\chi^{2}\right)$, is greater than 0.01. Figure 72 through Figure 74 show the track match efficiency for data and MC. The data distributions are not background subtracted and are used to parameterize the efficiencies vs probe track $p_{T}$. The parameterized efficiencies are calculated in data and MC using a $1 \mathrm{D}\left(p_{T}\right)$ fit for electrons with $\left|\eta_{\text {det }}\right|<1.1$, and a 2D $\left(p_{T}, \eta\right)$ fit for the forward electrons. Table XVI and Figure 75 show the average electron-track match efficiency versus jet multiplicity after background subtraction in data.

The sidebands to the di-em invariant mass peak are used to subtract the background for determining the average track match efficiency. The di-em invariant mass distributions are also fitted with a convoluted Breit-Wigner and Gaussian fit for the $Z$ boson mass region with an exponential shape for the background. The agreement between the two background subtracting techniques is found to be within $1 \%$.

The uncertainty for the track match scale factor is estimated from four sources. The first source is the statistical uncertainty of the average efficiency value for $Z+\geq 0 j: 0.3 \%$. The second source is the difference between the $Z+\geq 0 j$ average efficiency value and the $Z+\geq 2 j$ average efficiency value in data: $0.9 \%$. The third source is coming from the errors of the exponential fit for the background subtraction of the $Z$ mass region: $0.4 \%$. The fourth source is the difference between the MC $Z+\geq 0 j$ average efficiency value and the MC $Z+\geq 2 j$ average efficiency value: $+0.8 \%$. The first three sources of uncertainty are added in quadrature, and the 
fourth source is added linearly. The overall track match uncertainty is $\pm 1.0_{-0}^{+0.8 \%}$ and shown in Table XVII.

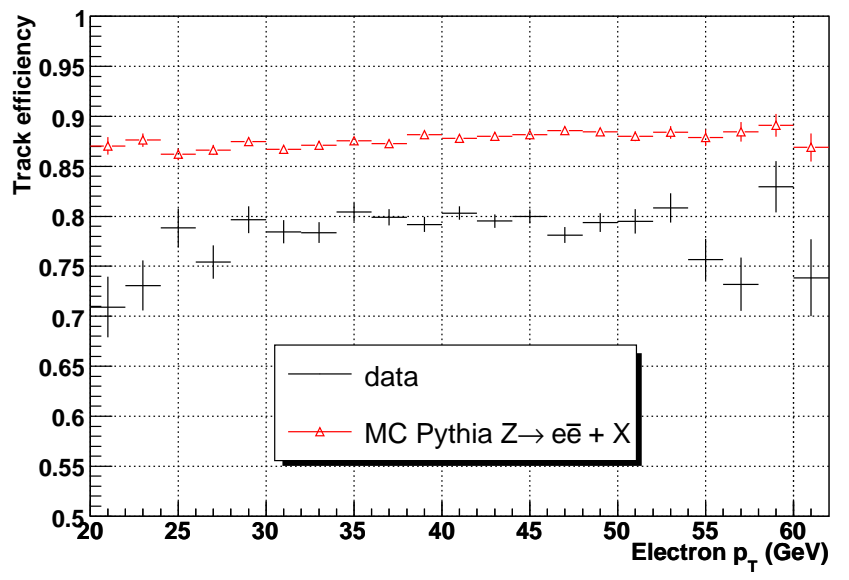

Figure 72. Electron-track match efficiencies in data and $\mathrm{MC}$ for the $Z$ inclusive sample vs probe electron $p_{T}$ without background subtraction. The MC sample is PYTHIA generated $Z \rightarrow e \bar{e}+X$. The probe electron is in the central region $\left|\eta_{\text {det }}\right|<1.1$ of the calorimeter. 


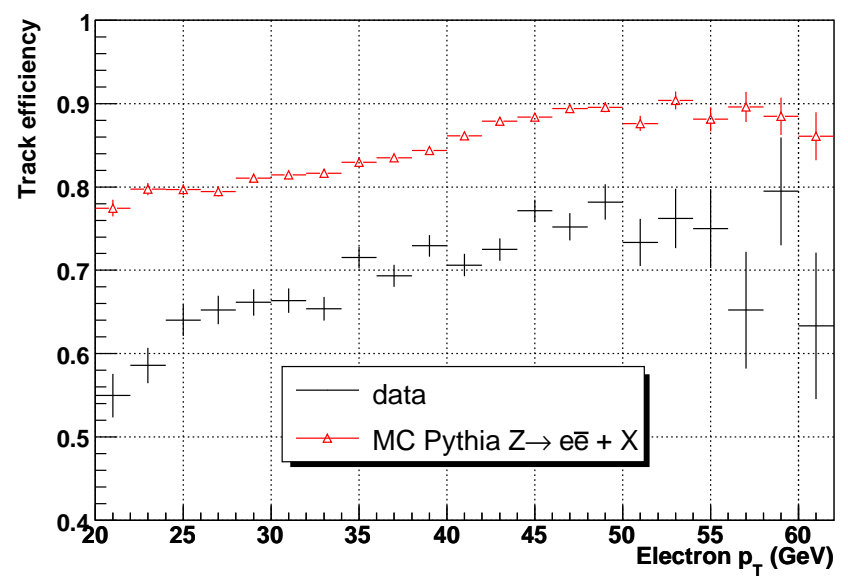

Figure 73. Electron-track match efficiencies in data and MC for the $Z$ inclusive sample vs probe electron $p_{T}$ without background subtraction. The MC sample is PYTHIA generated $Z \rightarrow e \bar{e}+X$. The probe electron is in the forward region $1.5<\left|\eta_{\text {det }}\right|<2.5$ of the calorimeter.

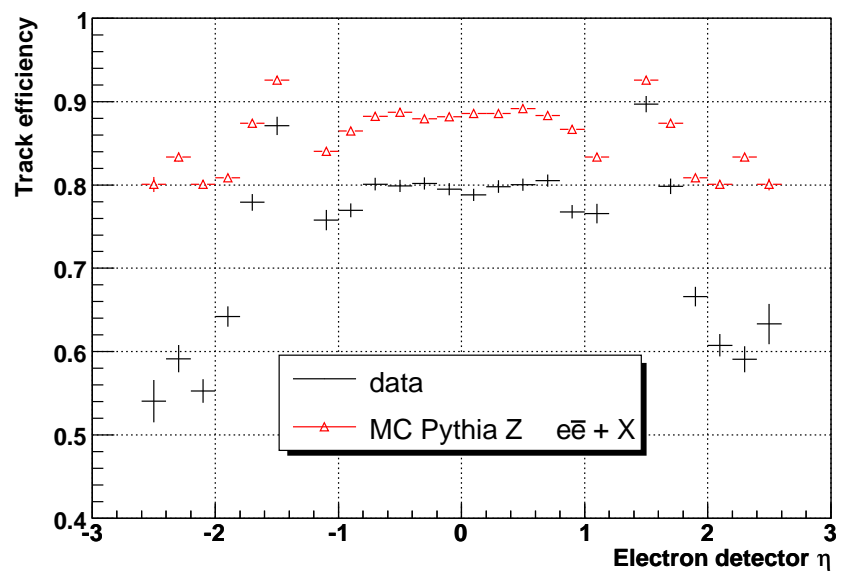

Figure 74. Electron-track match efficiencies in data and MC for the $Z$ inclusive sample vs probe electron $\eta_{\text {det }}$ without background subtraction. The MC sample is PYTHIA generated $Z \rightarrow e \bar{e}+X$. 


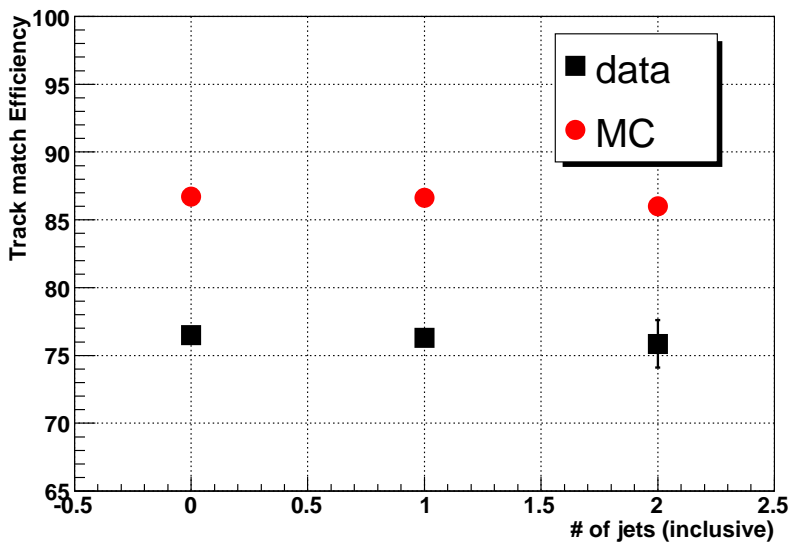

Figure 75. Electron-track match efficiencies vs jet multiplicity for data and MC. The background in data has been removed by using the sidebands of the invariant mass peak.

\begin{tabular}{|c|c|c|c|}
\hline sample & $>=0$ jets & $>=1$ jet & $>=2$ jets \\
\hline data & $76.51 \pm 0.20$ & $76.31 \pm 0.62$ & $75.85 \pm 1.75$ \\
Monte Carlo & $86.70 \pm 0.07$ & $86.63 \pm 0.21$ & $86.00 \pm 0.69$ \\
\hline
\end{tabular}

TABLE XVI

BACKGROUND SUBTRACTED ELECTRON-TRACK MATCH EFFICIENCIES VS JET MULTIPLICITY FOR DATA AND MC. THE UNCERTAINTIES ARE STATISTICAL. 


\begin{tabular}{|c|c|}
\hline Uncertainty due to & $\%$ \\
\hline Statistical & 0.3 \\
Avg eff data $Z+\geq 0 j$ - Avg eff data $Z+\geq 2 j$ & 0.9 \\
Background exponential fit uncertainty & 0.4 \\
Avg eff MC $Z+\geq 0 j$ jet - Avg eff MC $Z+\geq 2 j$ & +0.8 \\
\hline Overall & $\pm 1.0_{-0}^{+0.8}$ \\
\hline
\end{tabular}

TABLE XVII

ELECTRON-TRACK MATCH UNCERTAINTIES. 


\subsection{Jet Reconstruction and Identification Efficiency}

The jet reconstruction and identification (RECO/ID) efficiency is estimated using a tuned MC sample according to the following procedure (74).

- A scale factor is derived based on the " $Z p_{T}$ balance" method. This method selects events with $Z$ candidates and probes for a recoiling jet opposite in $\phi$. The probability of finding a recoiling jet can be measured as a function of $Z p_{T}$ in data and MC.

- The ratio of the $Z p_{T}$ probability in data and MC yields a scale factor.

- The scale factor is applied to the MC sample to tune it to match the data distributions.

- The tuned MC sample is used to measure the RECO/ID efficiency by matching particle level jets with calorimeter jets within a search cone of $\Delta \mathrm{R}=0.4$.

- The RECO/ID efficiency is parameterized versus particle jet $p_{T}$. The $p_{T}$ values of the particle jets are smeared with the data energy resolutions.

The following sections discuss the derivation of the $Z p_{T}$ balance scale factor and the jet RECO/ID efficiency parameterization and uncertainties.

\subsection{1 " $Z p_{T}$ balance" scale factor}

The " $Z p_{T}$ balance" method selects events with $Z$ candidates and probes for a recoiling jet opposite in $\phi$. The following selection cuts are used to measure the probability of finding a recoiling jet as a function of $Z p_{T}$.

The following selection cuts are imposed on the EM objects before calculating their di-em invariant mass: 
- $p_{T}>25.0 \mathrm{GeV}$;

- $\left|\eta_{\text {det }}\right|<1.1$;

- EM Fraction > 0.90;

- Isolation $<0.15$

- $\chi^{2}$ of H-matrix $7<12.0$;

- Track match with $P\left(\chi^{2}\right)>0.01$ (using $\Delta \phi, \Delta \eta$, and $E / p$ );

- opposite sign tracks;

- Tracks must originate from the same primary vertex.

An invariant mass window of $80 \mathrm{GeV}<M_{e e}<100 \mathrm{GeV}$ is applied to the two leading electrons that satisfied the above cuts.

The following selection cuts are imposed on the jets:

- $p_{T}>15.0 \mathrm{GeV}$;

- $|\eta|<2.5$;

- $0.05<$ EM Fraction $<0.95$;

- Coarse hadronic fraction $<0.40$;

- Hot fraction $<10$;

- $\mathrm{n} 90>1$

- L1 confirmation $>0.40$. 
The jet with a $\Delta \phi$ separation of $\left|\Delta \phi_{Z, j e t}\right|>7 \pi / 12$ from the $Z$ boson is considered a match. The probability of finding a jet recoiling off of the $Z$ boson is estimated by dividing two histograms. The denominator histogram is the $p_{T}$ distribution of the $Z$ boson. The numerator histogram is the $p_{T}$ distribution of the $Z$ boson if a jet was found. A " $Z p_{T}$ balance" scale factor (Figure 76) is parameterized (Equation 8.2) after dividing the data and MC jet probability distributions. The uncertainty of the scale factor (Equation 8.3 and Equation 8.4) include the contributions from the error matrix of the fit and the variation of the scale factor when applying a $E_{T}<15 \mathrm{GeV}$ requirement, to eliminate $W^{ \pm}+$jets background events.

$$
\text { Scale Factor }=1.00 * \operatorname{Erf}\left(0.0285 * Z p_{T}+0.262\right)
$$

Upper band scale factor error $=0.0199+0.0750 * \operatorname{Exp}\left(1.97-0.0826 * Z p_{T}\right)$

Lower band scale factor error $=0.0172+0.217 * \operatorname{Exp}\left(-0.0503 * Z p_{T}\right)$

\subsubsection{Jet RECO/ID Efficiency}

The jet RECO/ID efficiency is measured by matching particle level jets with calorimeter jets within a search cone of $\Delta \mathrm{R}=0.4$. Figure 77 shows the matching efficiency of particle level

jets with calorimeter jets in three separate MC simulations without applying the $Z p_{T}$ scale factor: PYTHIA $\mathrm{Z}\left(\rightarrow \mathrm{e}^{+} \mathrm{e}^{-}\right)+\mathrm{X}$, ALPGEN + PYTHIA $\mathrm{Z}\left(\rightarrow \mathrm{e}^{+} \mathrm{e}^{-}\right) \mathrm{j}$ combined with $\mathrm{Z}\left(\rightarrow \mathrm{e}^{+} \mathrm{e}^{-}\right) \mathrm{jj}$, and an inclusive QCD sample. Good agreement in the jet efficiency is observed among these three MC simulations. 
The scale factor is applied to the MC and the jet RECO/ID efficiency is measured versus particle jet $p_{T}$, smeared with the jet energy resolution in data. These RECO/ID efficiencies are labeled as "data" efficiencies. The jet RECO/ID efficiency is also measured in MC versus smeared particle jet $p_{T}$ without applying the scale factor. The ratio of the "data" / MC" jet RECO/ID efficiencies versus smeared particle jet $p_{T}$ defines the scale factor that is applied to the $\mathrm{MC}$ jets in this analysis to simulate the data efficiency.

The uncertainty of the Jet RECO/ID in MC is estimated using the error matrix of the fit. The uncertainty of the Jet RECO/ID in "data" is estimated using the error matrix of the fit and the uncertainty from the " $Z p_{T}$ " balance scale factor.

Figure 78 through Figure 83 show the measured efficiencies in data and MC in three regions: $|\eta|<0.7,0.7<|\eta|<1.5$, and $1.5<|\eta|<2.5$. The parameterizations of the efficiency curves are shown in Table XVIII with $\pm 1 \sigma$ statistical and systematic uncertainties in Table XIX. Figure 84 and Figure 86 compares "data" efficiency using the " $Z p_{T}$ balance" scale factor without smearing the particle jets to the jet energy resolution in data to an efficiency using a "Photon $p_{T}$ balance" method that was measured in data (75). 


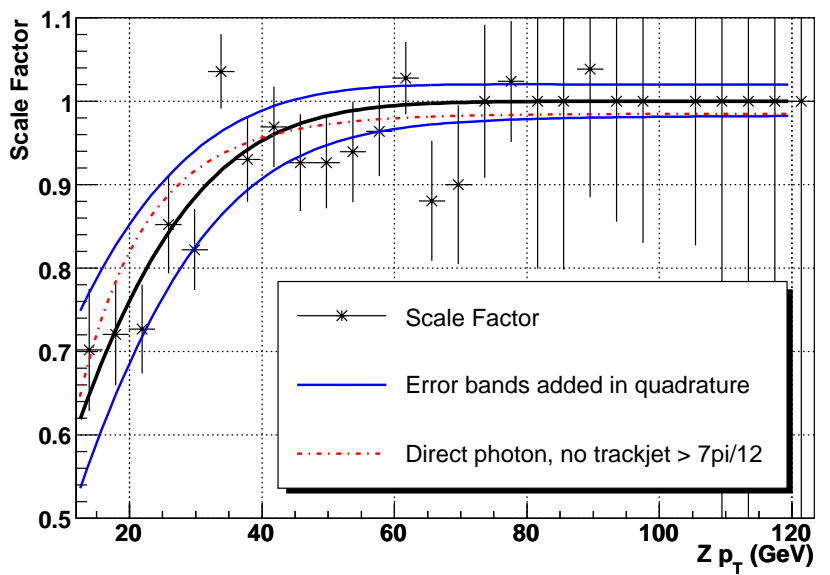

Figure 76. Scale factor (solid black line) vs $Z p_{T}$ overlaid with direct photon scale factor (in dashed line). $\pm 1 \sigma$ uncertainty (thinner blue lines) bound the scale factor.

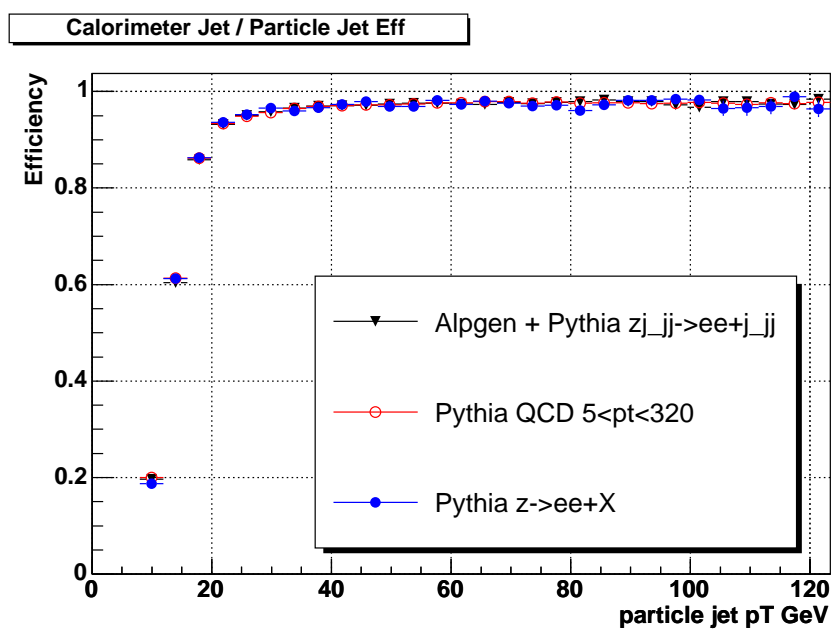

Figure 77. The efficiency to find a calorimeter jet given a particle jet in three separate MC simulations. 


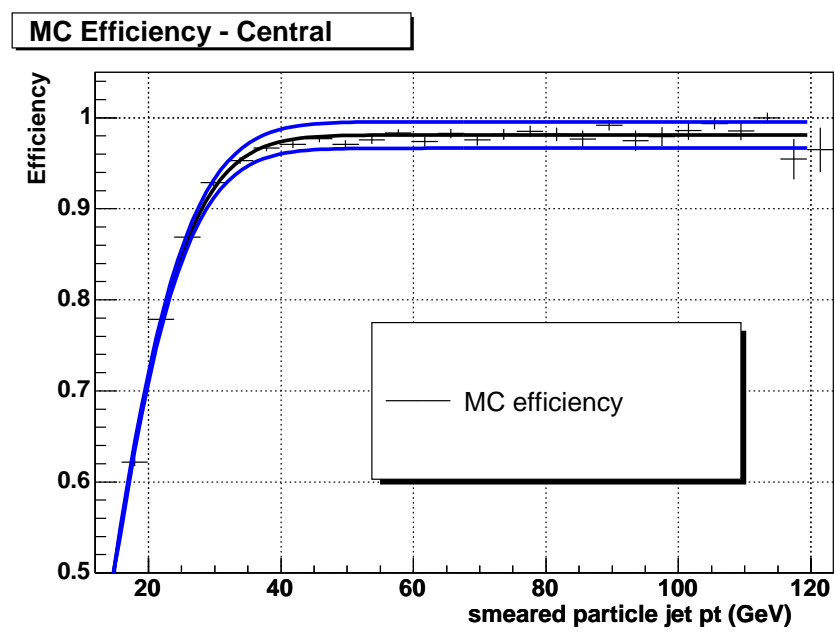

Figure 78. Jet RECO/ID efficiency in MC for $|\eta|<0.7$ vs smeared particle jet $p_{T}$. The three lines represent the fit to the data (central line) and the $\pm 1 \sigma$ uncertainties.

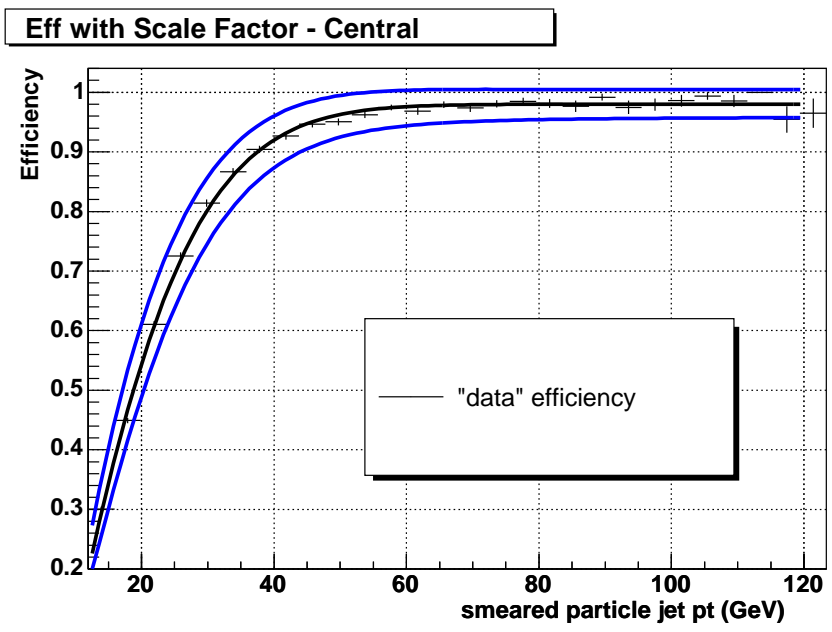

Figure 79. Jet RECO/ID efficiency in "data" for $|\eta|<0.7$ vs smeared particle jet $p_{T}$. The three lines represent the fit to the data (central line) and the $\pm 1 \sigma$ uncertainties. 


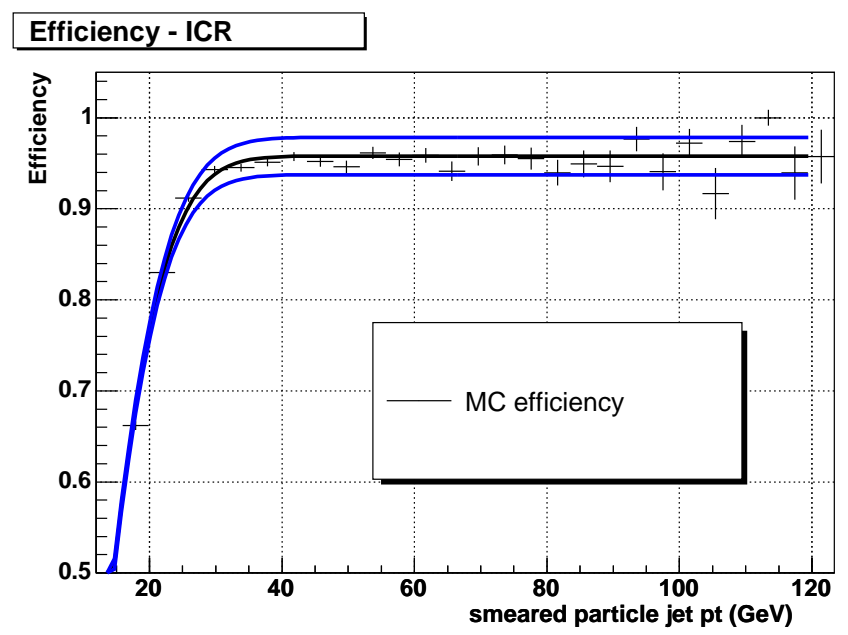

Figure 80. Jet RECO/ID efficiency in MC for $0.7<|\eta|<1.5$ vs smeared particle jet $p_{T}$. The three lines represent the fit to the data (central line) and the $\pm 1 \sigma$ uncertainties.

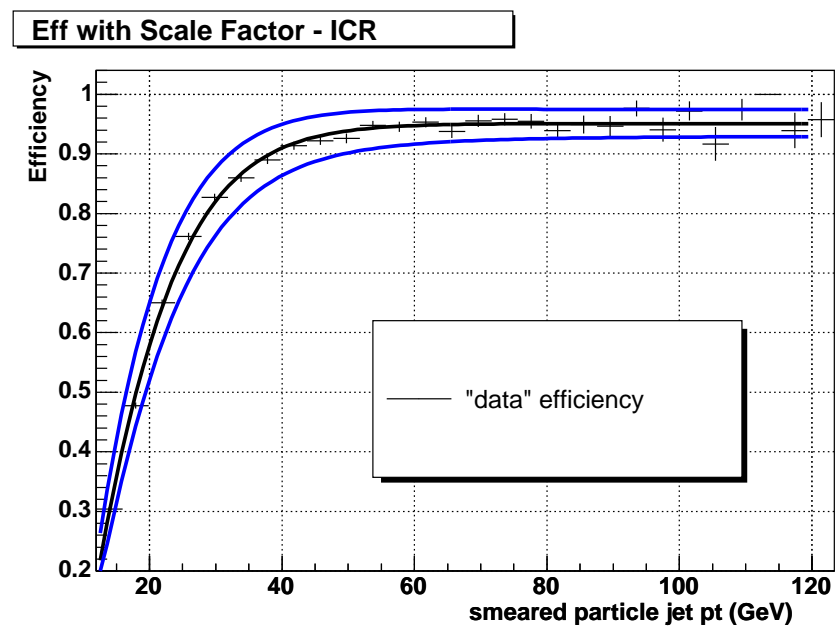

Figure 81. Jet RECO/ID efficiency in "data" for $0.7<|\eta|<1.5$ vs smeared particle jet $p_{T}$. The three lines represent the fit to the data (central line) and the $\pm 1 \sigma$ uncertainties. 


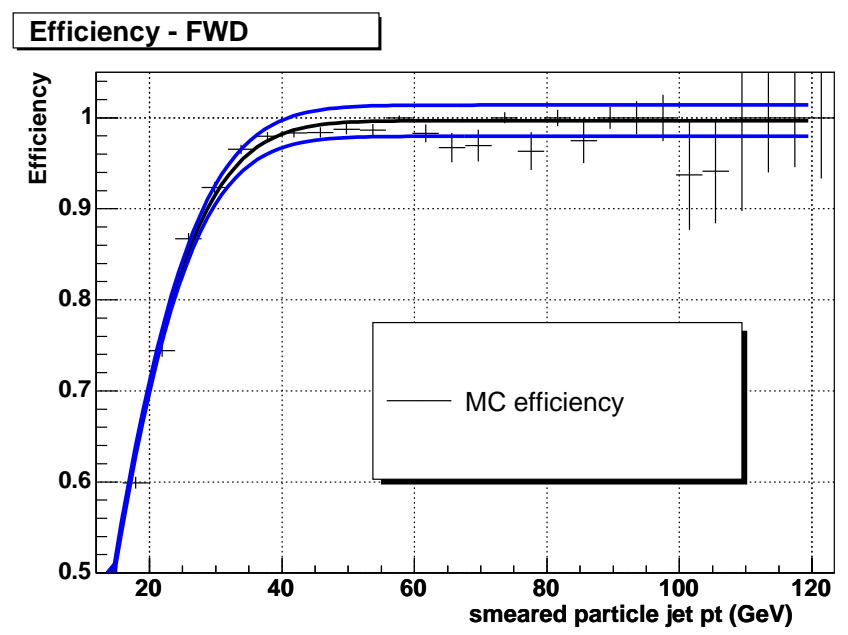

Figure 82. Jet RECO/ID efficiency in MC for $1.5<|\eta|<2.5$ vs smeared particle jet $p_{T}$. The three lines represent the fit to the data (central line) and the $\pm 1 \sigma$ uncertainties.

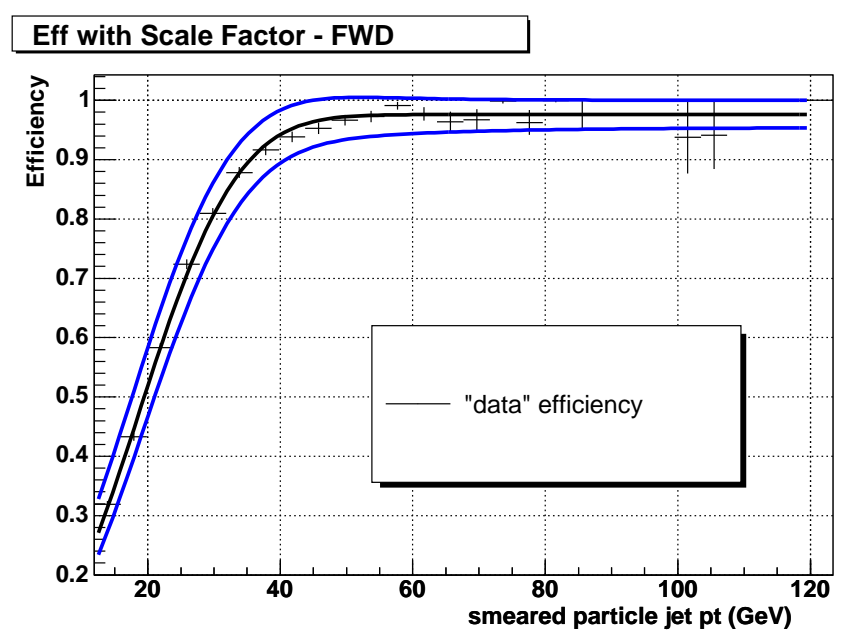

Figure 83. Jet RECO/ID efficiency in "data" for $1.5<|\eta|<2.5$ vs smeared particle jet $p_{T}$. The three lines represent the fit to the data (central line) and the $\pm 1 \sigma$ uncertainties. 


$p 0 * \operatorname{Erf}(p 1+p 2 * x)$
\begin{tabular}{|lllll|}
\hline Eta range & $\mathrm{p} 0$ & $\mathrm{p} 1$ & $\mathrm{p} 2$ \\
\cline { 2 - 6 } & $\mathrm{MC}\left|\eta_{\text {jet }}\right|<2.5$ & 0.972 & -0.388 & 0.0601 \\
$\mathrm{MC}\left|\eta_{\text {jet }}\right|<0.7$ & 0.981 & -0.340 & 0.0559 \\
$\mathrm{MC} 0.7<\left|\eta_{\text {jet }}\right|<1.5$ & 0.958 & -0.573 & 0.0739 \\
\hline \multicolumn{5}{|c|}{$p 0 * \operatorname{Erf}\left(p 1 * x+p 2 * x^{1 / 2}+p 3 * x^{1 / 4}\right)$} \\
\hline MC $1.5<\left|\eta_{\text {jet }}\right|<2.5$ & 0.991 & 0.194 & -1.87 & 2.46 \\
\hline Eta range $p 0 * \operatorname{Er} f\left(p 1 * x+p 2 * x^{1 / 2}+p 3 * x^{1 / 4}\right)$ \\
\hline Data $\left|\eta_{\text {jet }}\right|<2.5$ & 0.964 & 0.0294 & 0.279 & -0.605 \\
Data $\left|\eta_{\text {jet }}\right|<0.7$ & 0.980 & 0.0131 & 0.466 & -0.854 \\
Data $0.7<\left|\eta_{\text {jet }}\right|<1.5$ & 0.951 & -0.0137 & 0.925 & -1.54 \\
Data $1.5<\left|\eta_{\text {jet }}\right|<2.5$ & 0.976 & 0.0997 & -0.729 & 0.841 \\
\hline
\end{tabular}

TABLE XVIII

THE MC AND "DATA" JET RECO/ID EFFICIENCY AS A FUNCTION OF THE PARTICLE JET $P_{T}$ SMEARED WITH THE JET ENERGY RESOLUTIONS FROM DATA. 


\begin{tabular}{|c|c|c|c|c|}
\hline \multicolumn{5}{|c|}{$p 0+p 1 *(x-p 2)^{2}$} \\
\hline Error band & p0 & $\overline{\mathrm{p} 1}$ & & $\mathrm{p} 2$ \\
\hline MC Upper and Lower & $4.59 * 10$ & $\begin{array}{ll}-3 & 1 .\end{array}$ & $7 * 10^{-6}$ & 18.3 \\
\hline \multicolumn{5}{|c|}{$p 0+p 1 * \operatorname{Exp}(p 2 * x)$} \\
\hline Eta range & $\overline{\mathrm{p} 0}$ & $\mathrm{p} 1$ & $\mathrm{p} 2$ & \\
\hline Data Upper Error & 0.0374 & 0.500 & -0.0896 & \\
\hline Data Lower Error & 0.0235 & 0.239 & -0.0570 & \\
\hline
\end{tabular}

TABLE XIX

THE MC AND "DATA" JET RECO/ID EFFICIENCY ERRORS AS A FUNCTION OF THE PARTICLE JET $P_{T}$ SMEARED WITH THE JET ENERGY RESOLUTIONS FROM DATA. 


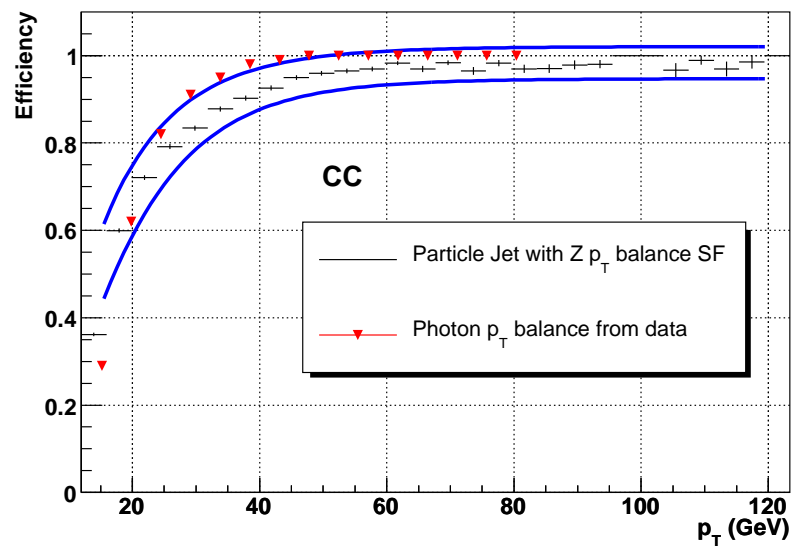

Figure 84. The data points with $\pm 1 \sigma$ uncertainty bands for the jet RECO/ID efficiency in "data" for $|\eta|<0.7$ without smearing the particle jets to the energy resolution of the calorimeter jets in data. The inverted triangle shows efficiencies derived with the photon balance technique measured in data. 


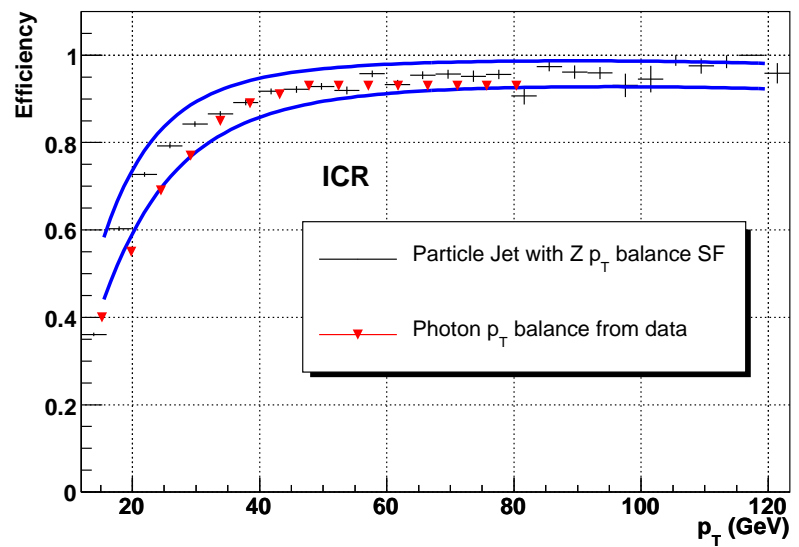

Figure 85. The data points with $\pm 1 \sigma$ uncertainty bands for the jet RECO/ID efficiency in "data" for $0.7<|\eta|<1.5$ without smearing the particle jets to the energy resolution of the calorimeter jets in data. The inverted triangle shows efficiencies derived with the photon balance technique measured in data.

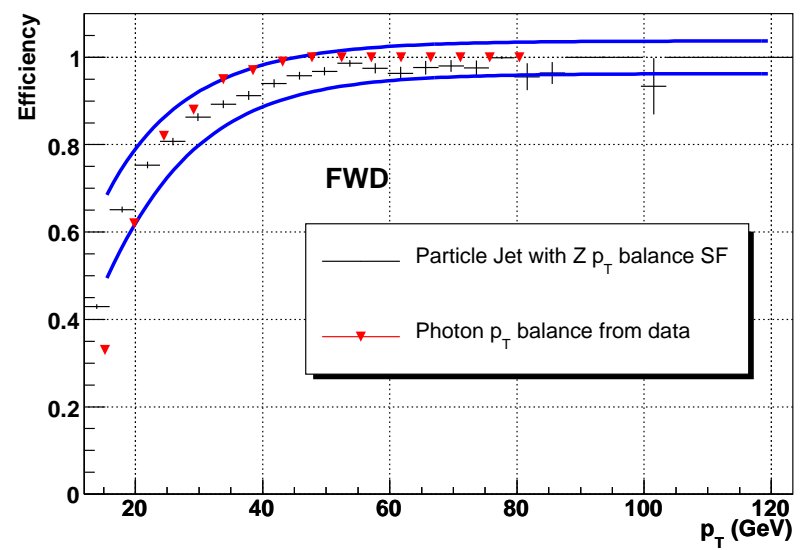

Figure 86. The data points with $\pm 1 \sigma$ uncertainty bands for the jet RECO/ID efficiency in "data" for $1.5<|\eta|<2.5$ without smearing the particle jets to the energy resolution of the calorimeter jets in data. The inverted triangle shows efficiencies derived with the photon balance technique measured in data. 


\subsection{Jet Taggability Efficiencies}

Jet taggability efficiencies are determined in data and MC. The efficiencies are measured using events with a $Z$ boson and at least one calorimeter jet. Using all the jets in these events, the number of taggable jets divided by the number of calorimeter jets is determined as a function of jet $p_{T}$ and $\eta$. Figure 87 and Figure 88 show the taggability efficiencies for MC and data. The average efficiency in data is $78.3 \pm 0.7 \%$. A comparison of the average jet taggability efficiency measured in data to a variety of MC samples is shown in Figure 89. The MC samples with b-quark final states have an enhanced taggability probability due to the B hadrons producing higher multiplicity of tracks in jets than light-quark initiated jets.

The uncertainty on the jet taggability scale factor is estimated by fitting the statistical uncertainty distributions from the data measurement. The statistical uncertainties are parameterized versus jet $p_{T}$ and $\eta$. The statistical uncertainty in the $\eta$ distribution starts at $4 \%$ in the central region and grows to $10 \%$ in the forward regions. The statistical uncertainty in the $p_{T}$ distribution starts at $1.5 \%$ at $20 \mathrm{GeV}$ and grows to $9 \%$ at $100 \mathrm{GeV}$. 


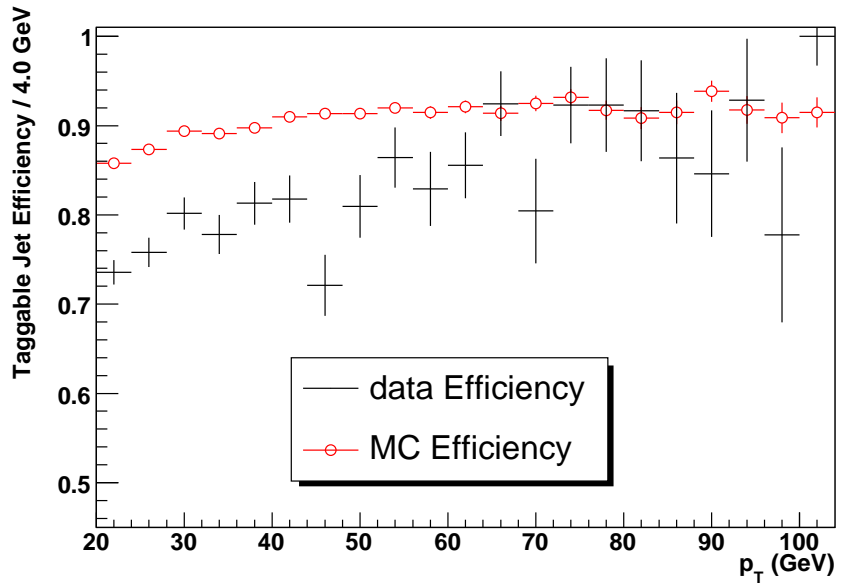

Figure 87. Taggable jet efficiency for data and MC for $|\eta|<2.5$ as a function of calorimeter jet $p_{T}$.

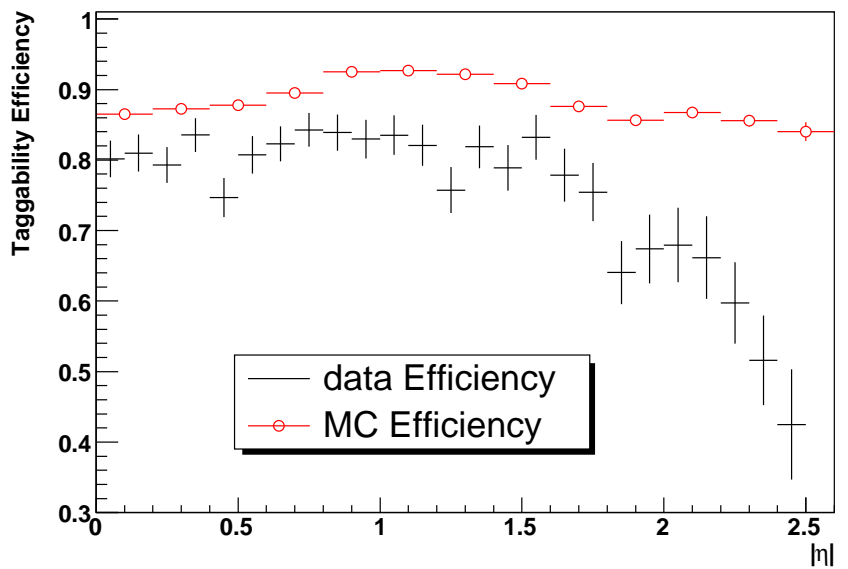

Figure 88. Taggable jet efficiency for data and MC within $|\eta|<2.5$ as a function of calorimeter jet $|\eta|$. 


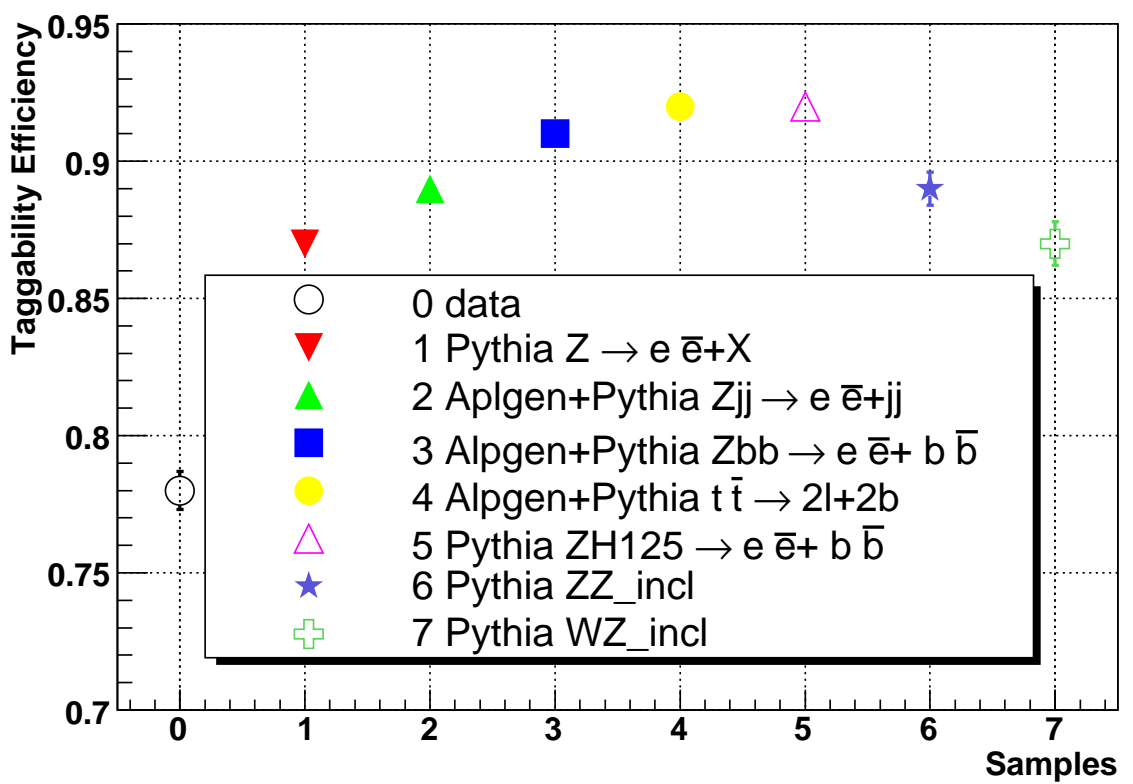

Figure 89. Average taggable jet efficiency of data and various MC samples. 


\section{6 b-jet Tagging Efficiencies}

The JLIP efficiency curves are measured in data by using two b-tagging algorithms (JLIP and $\mathrm{p}_{T}^{r e l}$ ), a system of eight equations with eight unknowns (81) (Appendix E), and a data jet sample enhanced with muons. The $\mathrm{p}_{T}^{r e l}$ b-tagging algorithm uses the transverse momentum of the muon relative to the jet axis. As the muons produced from light-quark initiated jets come from the primary vertex, b jets have large $\mathrm{p}_{T}^{r e l}$ values compared to light quark jets. For each calorimeter jet, a probability is computed using the impact parameter information of tracks seen in the SMT layers. The b-tagging efficiency is estimated by using real data with reconstructed muons inside the jets. The JLIP efficiency is parameterized as a function of taggable muonin-jet $p_{T}$ and $\eta$. MC muon-in-jet JLIP efficiencies are calculated in the same manner. The muon-in-jet scale factor is applied to an inclusive MC simulated jet sample which generates the efficiency curves (tagging rate functions) for inclusive taggable jets in data.

There are six JLIP selection cut values which are compared in the following figures. Figure 90 shows the tagging rate functions or b-tagging efficiencies for $\mathrm{b}$ jets in data. Figure 91 shows the tagging rate functions for $\mathrm{c}$ jets in data and the efficiency is roughly half of the b-tagging efficiencies. Figure 92 shows the tagging rate functions of light-quark jets (mis-tag efficiencies), which are roughly the JLIP cut value. Figure 93 shows the scale factor applied to the MC simulation.

The JLIP b-tagging rate $\pm 1 \sigma$ statistical and systematic uncertainties as a function of jet $p_{T}$ and $\eta$ are shown in Figure 94. The uncertainties are estimated from the efficiency variations when using different data samples, modifications of the assumed b- and c-quark contributions in 
QCD MC, and using different b quark to muon MC samples. Statistical uncertainties dominate at high $p_{T}$ and high $\eta$.

In this analysis, the MC taggable jets are considered b-tagged if their JLIP probability value was less than the JLIP selection cut value. A b-tagged jet is removed from the event if a randomly generated number is greater than the scale factor value that corresponds to this jet. Another technique, used in other analysis, uses all the MC taggable jets and independently removes them from the event if a randomly generated number is greater than the $\mathrm{TRF}_{b}$ for each jet. The JLIP cut value with scale factor efficiencies are overlaid with Tagging Rate Functions (TRFs) efficiencies in Figure 95 and Figure 96. These two plots are generated using an ALPGEN+PYTHIA $Z b \bar{b}$ sample. All jets are required to have a B hadron or meson within $\Delta R<0.4$ of the jet. The points without an additional marker represent the JLIP probability selection cut and scale factor. The points with a filled in circle represent the JLIP $\mathrm{TRF}_{b}$ on all of the taggable jets. The two techniques agree reasonably well. 

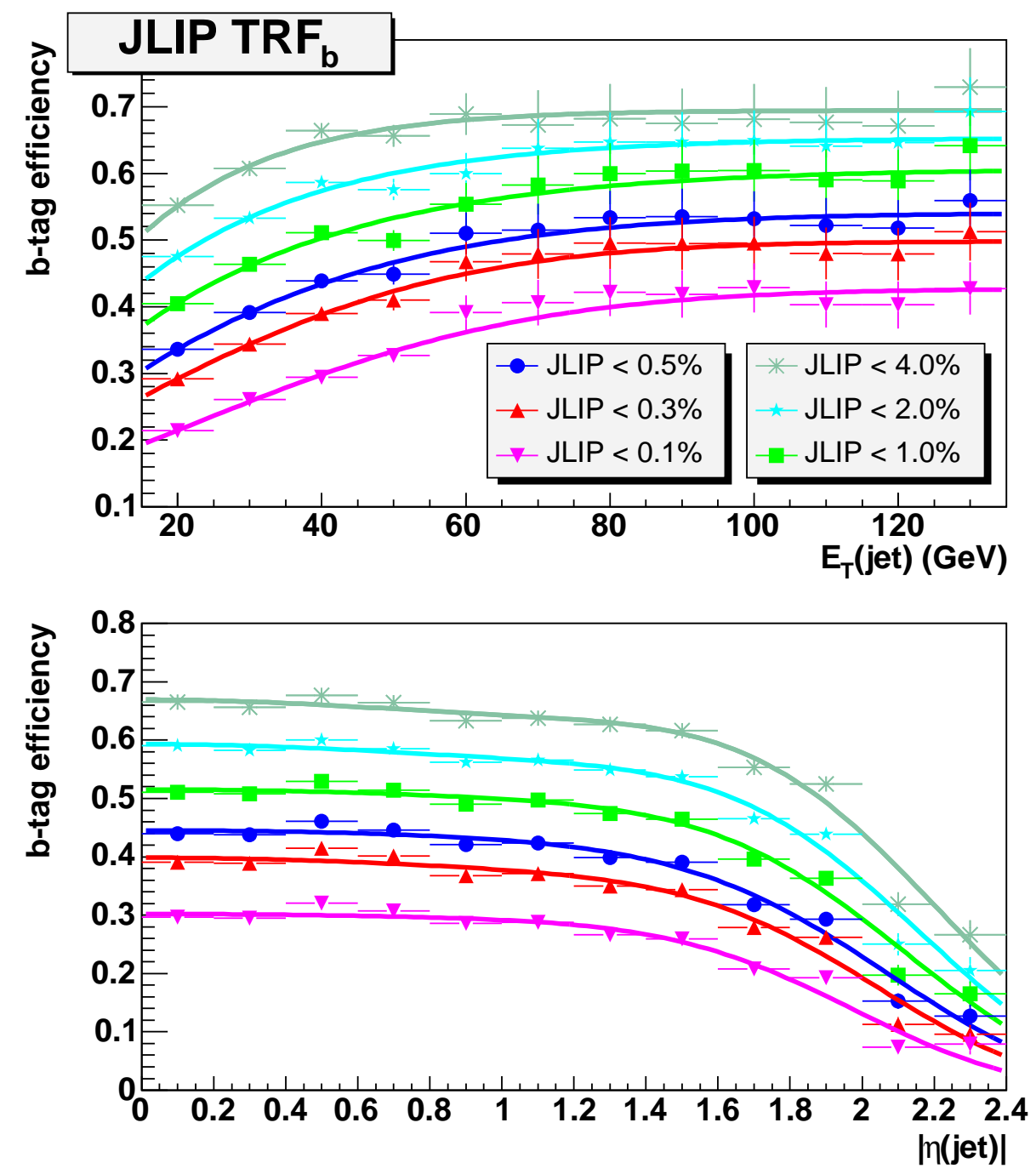

Figure 90. Tag Rate Function $T R F_{b}$ for simulated inclusive b-tag jets, versus $E_{T}$ and $|\eta|$ for all six working points. 

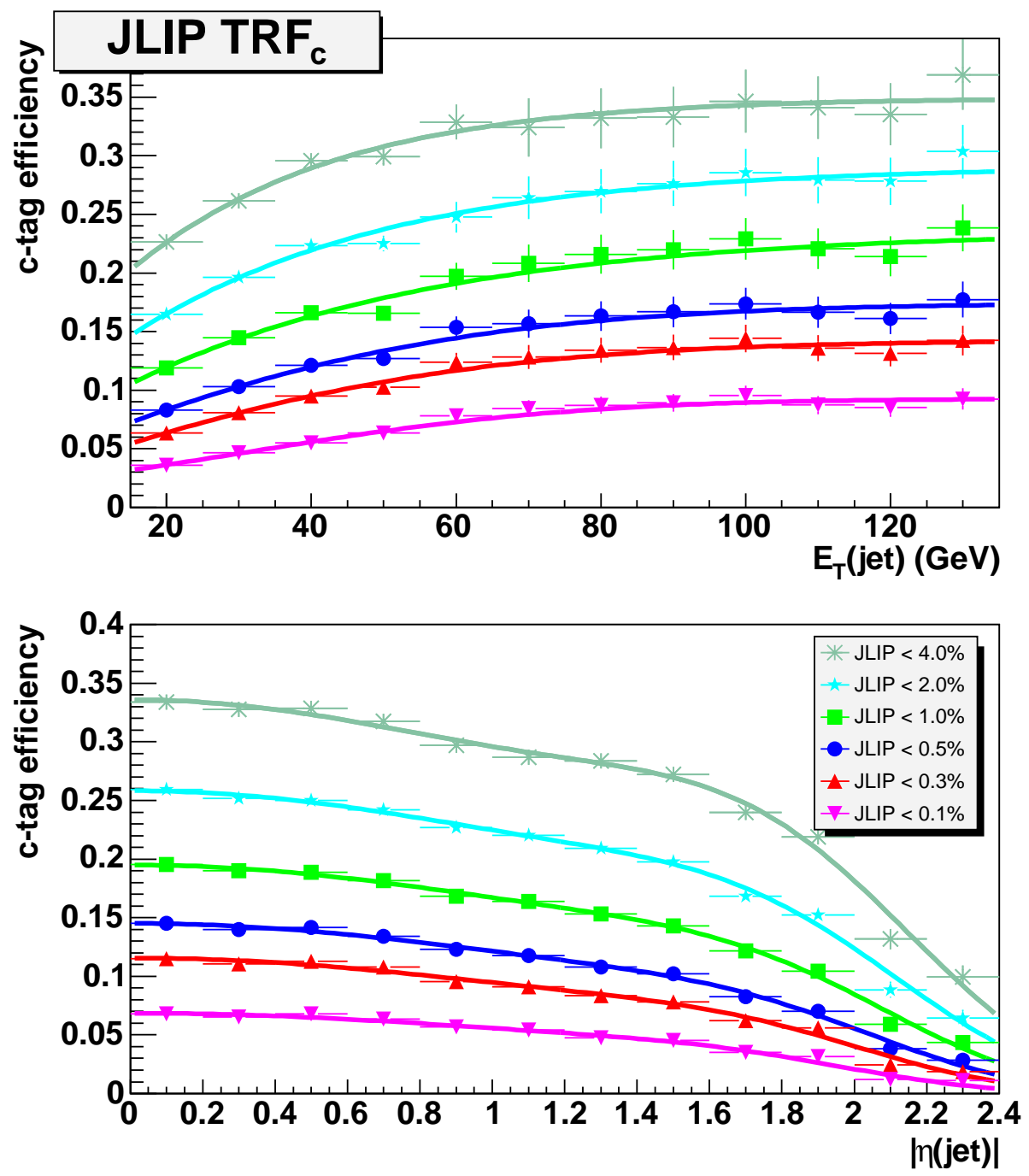

Figure 91. Tag Rate Function $T R F_{c}$ for simulated inclusive c-tag jets versus $E_{T}$ and $|\eta|$ for all six working points. 

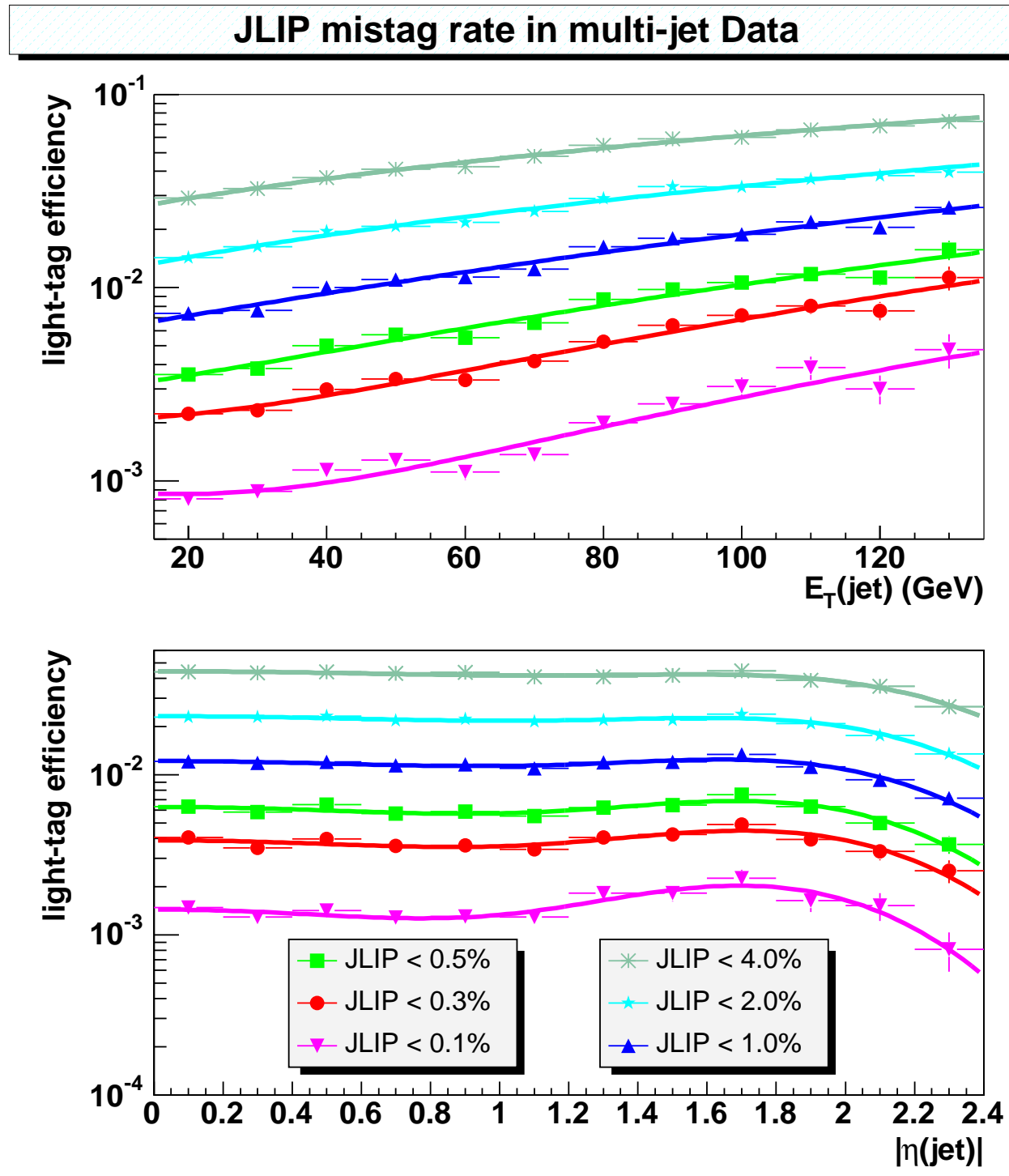

Figure 92. Light quark tagging efficiency for all six working points versus jet $E_{T}$ and $|\eta|$. 

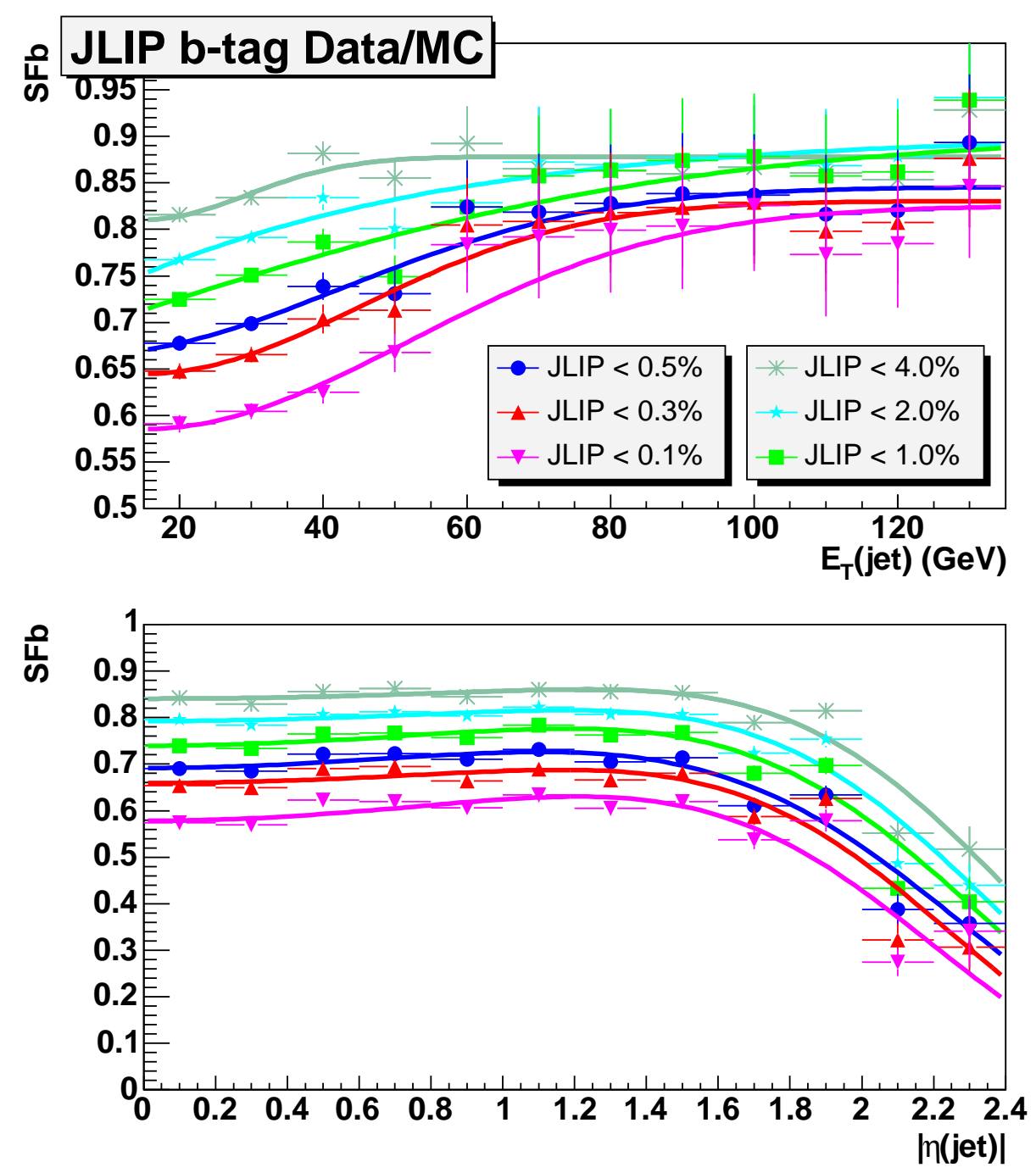

Figure 93. $S F_{b}$ scale factor, ratio of the b-tagging efficiencies measured in muon-in-jet data and simulation versus the jet $E_{T}$ and $|\eta|$ for all six working points. 


\section{JLIP TRFs : stat. $\oplus$ syst relative errors}
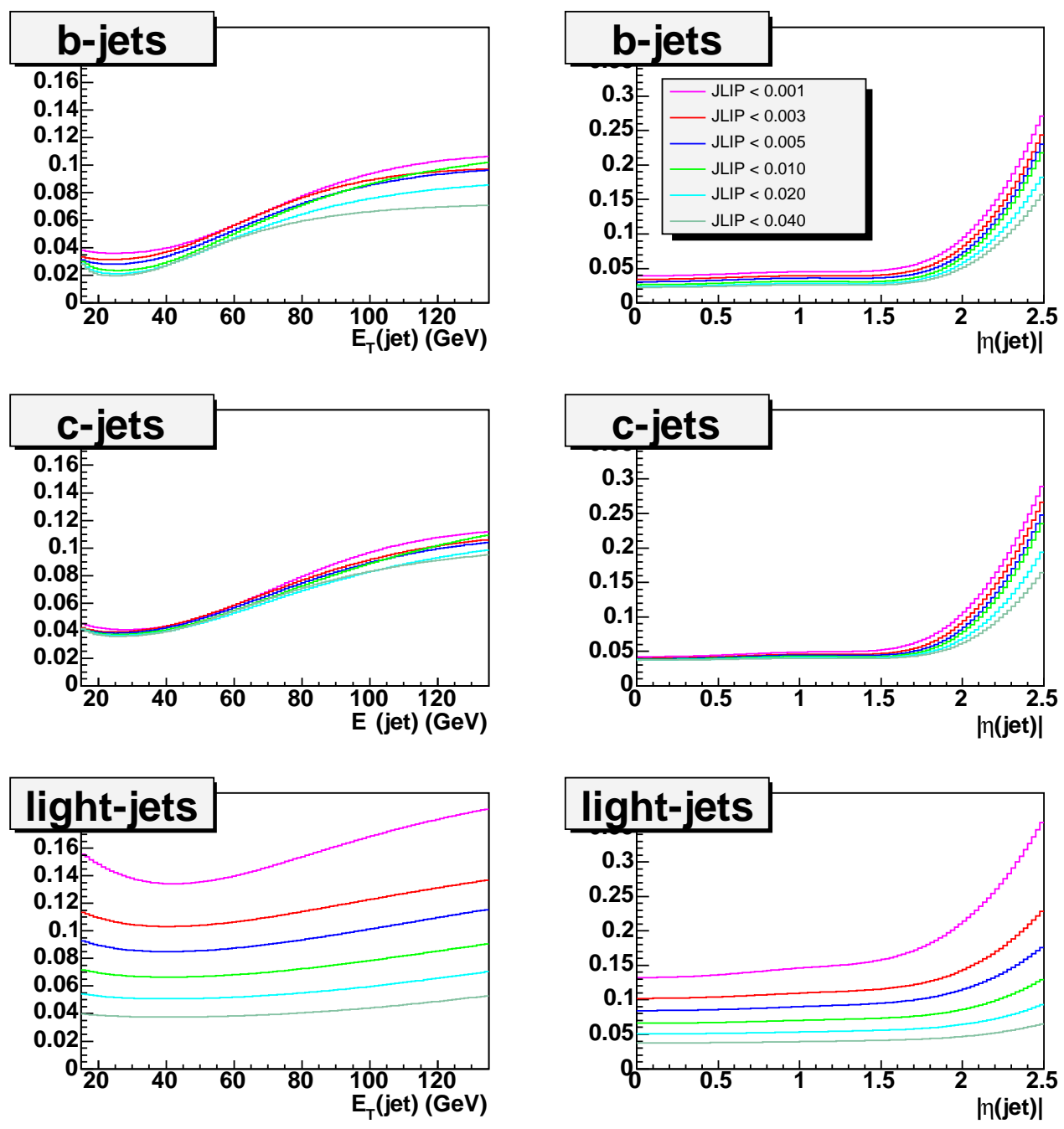

Figure 94. Overall relative uncertainties on $T R F_{b}, T R F_{c}$, and $T R F_{\text {light }}$ versus jet $E_{T}$ and $|\eta|$ for all working points. The statistical uncertainties and systematics are summed in quadrature. 


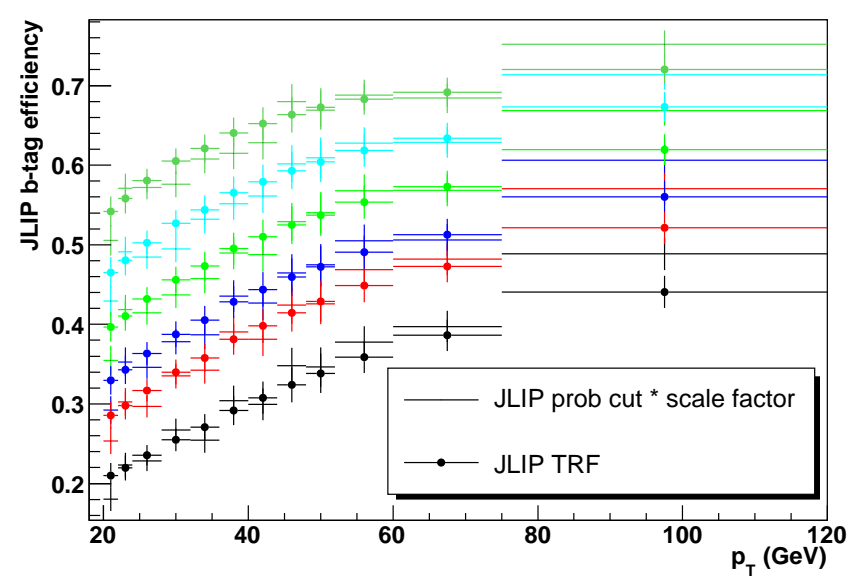

Figure 95. JLIP efficiencies for the six working points as a function of jet $p_{T}$. The solid lines use the MC JLIP probability and then the JLIP scale factor. The square points use the JLIP TRFs for b jets. The MC simulation is ALPGEN+PYTHIA $Z b \bar{b}$.

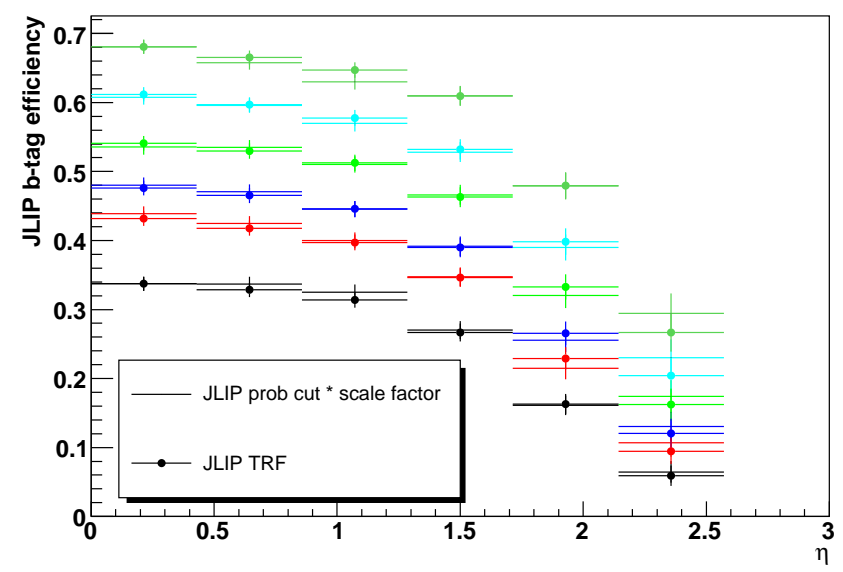

Figure 96. JLIP efficiencies for the six working points as a function of $|\eta|$. The solid lines use the MC JLIP probability and then the JLIP scale factor. The square points use the JLIP TRFs for b jets. The MC simulation is ALPGEN+PYTHIA $Z b \bar{b}$. 


\section{CHAPTER 9}

\section{HIGH LEVEL ANALYSIS}

A counting method is used in this analysis to search for the Higgs boson in the channel $Z\left(\rightarrow e^{+} e^{-}\right) H(\rightarrow b \bar{b})$. The number of events in data within a di-jet invariant mass window, which is centered on a hypothetical value for the mass of the Higgs boson, is compared to the number of events expected from background processes. Since there is a lack of signal events, a 95\% CL upper cross section limit of $Z H$ production is calculated. The following sections describe the high level analysis selection criteria, the comparisons of data to MC predictions, the Higgs signal region, and the contributions of the background processes to this search.

\subsection{High Level Analysis Cuts}

The following selection criteria are applied to the data and MC simulation after the electron and jet object identification selection cuts from Chapter 7 .

- $\mid$ Primary vertex $\mid<60 \mathrm{~cm}$ along the $\mathrm{Z}$ axis from the center of the detector;

- At least one electron candidate passes all levels of the trigger;

- At least two electrons with $p_{T}>20 \mathrm{GeV}$ within $\left|\eta_{\text {det }}\right|<1.1$ or $1.1<\left|\eta_{\text {det }}\right|<2.5$;

- At least one of the electrons is within $\left|\eta_{\text {det }}\right|<1.1$;

- At least one of the electrons has a track match;

- $75 \mathrm{GeV}<\mathrm{M}_{e e}<105 \mathrm{GeV}$; 
- At least two jets with $p_{T}>20 \mathrm{GeV}$ with $|\eta|<2.5$;

- At least two taggable jets;

- At least two b-tag jets or an exclusive single b-tag jet.

\subsection{Background Processes to the Higgs Signal}

The background processes that contribute to the $Z H$ search are discussed in this section. ALPGEN+PYTHIA and PYTHIA was used to generate events with final states that include electrons and jets processed through the full detector simulation. The simulated events are tuned to take into account the measured response of the DØ detector systems. The "luminosities" of the MC background processes are scaled so that the simulated rates match other observed or expected rates corresponding to events in a data sample of $452 \mathrm{pb}^{-1}$.

The luminosity scaling factor applied to the MC events is $L_{\text {data }} / L_{M C}$, where $L_{M C}$ is defined as:

$$
(\sigma \times B r)_{M C}=\frac{N}{L_{M C}}
$$

$\mathrm{N}$ is the initial number of generated events in each MC sample, $\sigma$ is the cross section given by the $\mathrm{MC}$ generator, and $\mathrm{Br}$ is the branching ratio of the process. The ALPGEN+PYTHIA $Z j j$ simulation is the only process in which the theoretical cross section is allowed to vary. The $Z j j$ simulation is normalized so that the total number of MC events equals to the total number of events observed in data.

Since the response of the detector is not perfectly modeled in the MC, scale factors are needed to account for the differences in efficiency for identifying electrons, tracks, jets, and 
finding taggable and b-jets. The differences and similarities between the MC background processes and the $Z\left(\rightarrow e^{+} e^{-}\right) H(\rightarrow b \bar{b})$ signal are described below.

\subsection{1 $\underline{\mathrm{Z}\left(\rightarrow e^{+} e^{-}\right) b \bar{b}}$}

The $\mathrm{Z}\left(\rightarrow e^{+} e^{-}\right) b \bar{b}$ channel has the same final state as the Higgs signal in the $\mathrm{ZH}$ channel. However, the two b-jets are not from decays of the Higgs boson and tend to have a lower di-jet invariant mass.

\subsection{2 $\underline{t \bar{t} \text { Production }}$}

$t \bar{t}$ produces final states which contribute to the backgrounds of the $Z H$ signal. The top quark decays to a $W^{ \pm}$and a b quark nearly $100 \%$ of the time. Therefore, there are two b quarks which might form a di-jet invariant mass around the Higgs mass of interest. Furthermore, if both $W$ bosons decay to electrons and neutrinos, then a di-em invariant mass might pass the $Z$ boson selection cut. Another background source is when one of the $W$ bosons decays into two jets. If either of these jets fluctuates electromagnetically, the jet can fake an electron which, with the electron from the other $W$, could form a di-em invariant mass within the $Z$ boson selection cut.

\subsection{3 $\underline{Z\left(\rightarrow e^{+} e^{-}\right) j \bar{j}}$}

The $\mathrm{Z}\left(\rightarrow e^{+} e^{-}\right) j \bar{j}$ channel has the same final state as the Higgs signal, except that the two jets are initiated by non-b quarks which at least one of them is tagged as a b jet.

\subsection{4 $Z Z$ Production}

The $Z Z$ production contributes to the background as one $Z$ boson could decay into two electrons and the other into jets which are mis-identified as b jets. 


\subsection{5 $W Z$ Production}

The $W Z$ production contributes to the background in a similar way as the $Z Z$ production. The $Z$ into two electrons and the $W$ into jets.

\subsubsection{Instrumental Background}

The instrumental background contribution is estimated by fitting the electron invariant mass around the $Z$ pole with a Breit-Wigner convoluted with a Gaussian distribution, and an exponential curve for the background. The events at the sidebands of the invariant mass peak include QCD background events, di-em contributions from the Drell-Yan (DY) process, and a

small $Z$ boson contribution. Since the DY component is part of the di-em signal, the QCD contribution needs to be disentangled from the DY component. Using the ALPGEN+PYTHIA MC sample, the percentage of DY events in $Z / \gamma^{*} \rightarrow e^{+} e^{-}$decays is estimated by fitting a Gaussian and Breit-Wigner shape to the $Z$ component and an exponential shape to the DY component.

Figure 97 shows the di-em object invariant mass distribution in data for a $Z$ with two or more jets. Figure 98 is the same distribution but for ALPGEN+PYTHIA $Z / \gamma^{*}(\rightarrow e \bar{e}) j j$. The DY plus QCD contribution is found to be $7.78 \pm 1.29 \%$ in the data with a di-em mass range of 75 $<M_{e e}<105 \mathrm{GeV}$. The DY contribution is found to be $1.79 \pm 0.14 \%$ in the mass range of $75<$ $\mathrm{M}_{e e}<105 \mathrm{GeV}$ for the $Z / \gamma^{*}+\geq 2 j$ sample. The DY contribution in the data and the number of QCD events, $\mathrm{N}_{Q C D}$ is given by Equation 9.5.

$$
N_{(D Y+Q C D)}=N_{D Y}+N_{Q C D}
$$




$$
\begin{gathered}
R_{\left(M C_{\frac{D Y}{Z+D Y}}\right)}=\frac{N_{M C_{D Y}}}{N_{M C_{(Z+D Y)}}} \\
N_{D Y}=R_{M C \frac{D Y}{Z+D Y}}\left(N_{(Z+D Y+Q C D)}-N_{Q C D}\right) \\
N_{Q C D}=\frac{N_{(D Y+Q C D)}-R_{\left(M C_{\frac{D Y}{Z+D Y}}\right)} N_{(Z+D Y+Q C D)}}{\left.1-R_{\left(M C \frac{D Y}{Z+D Y}\right.}\right)}
\end{gathered}
$$

The QCD contribution in data is found to be $6.10 \pm 1.46 \%$ of the $Z$ bosons candidates in the di-em invariant mass signal window. There are $463 Z / \gamma^{*}+\geq 2 j$ in data, which include 28 QCD background events.

To compare distributions between MC and data, the contributions from QCD background under the $Z$ boson mass region should be taken into account. A QCD enhanced sample was constructed and normalized to the 28 background QCD events estimated in data. The QCD enhanced sample is generated in data using the EM1TRK skim. The object selection criteria is basically the same as for the electrons, except the $\chi^{2}$ of H-Matrix cut is inverted and the track match is dropped to enhance the sample with jets with large EM components.

The selection criteria are:

- $\mathrm{ID}=10$ or \pm 11 ;

- EM Fraction > 0.9;

- Isolation $<0.15$

- $\chi^{2}$ of $\mathrm{H}-\operatorname{Matrix}(7)>12$ if $\left|\eta_{\text {det }}\right|<1.1$;

- $\chi^{2}$ of H-Matrix(7) $>20$ if $1.5<\left|\eta_{\text {det }}\right|<2.5$;

- $p_{T}>20$ 
- No fiducial restrictions in $\phi$;

- At least one electron object has $\left|\eta_{\text {det }}\right|<1.1$;

- No track match requirements.

A QCD enhanced sample should not exhibit a $Z$ boson mass peak in the invariant mass distribution. Figure 99 and Figure 100 show the "di-em" fake objects invariant mass distribution for the QCD enhanced sample which uses the same convoluted Breit-Wigner plus Gaussian fit with an exponential curve for the background for $Z+\geq 0 j$ and $Z+\geq 2 j$ event samples, respectively. There is no $Z$ boson peak observed, and this QCD enhanced sample is used to account for the QCD contribution in the $Z+\geq 2 j$ sample.

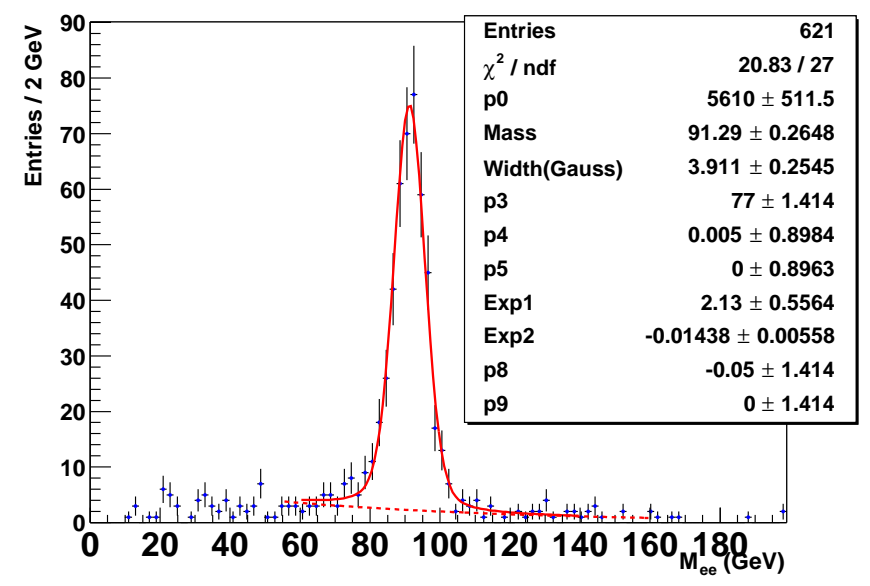

Figure 97. Di-electron invariant mass distribution for events with 2 or more jets in data. The fit is a convoluted Gaussian and Breit-Wigner with an exponential curve for the background. 


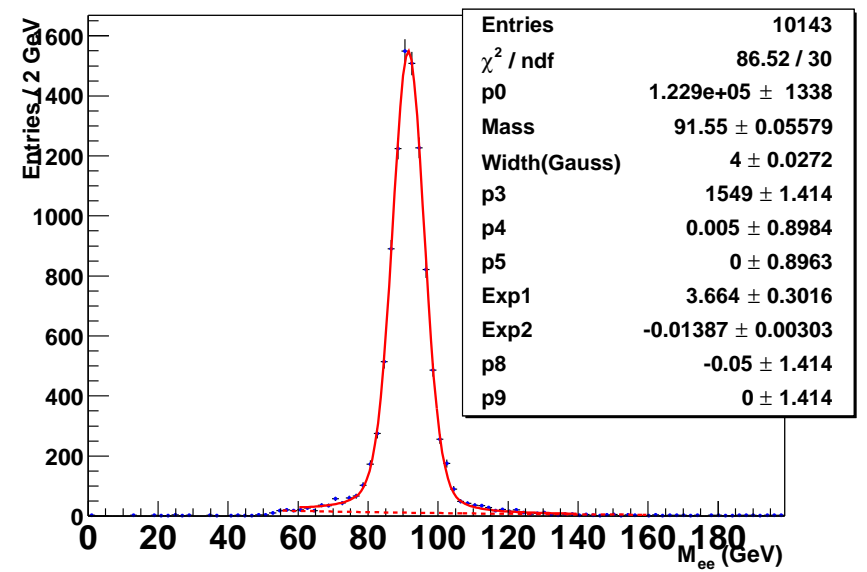

Figure 98. Di-electron invariant mass distribution for events with 2 or more jets in ALPGEN+PYThia MC simulated $Z j j \rightarrow e \bar{e}+j j$. The fit is a convoluted Gaussian and Breit-Wigner with an exponential curve for the background. 


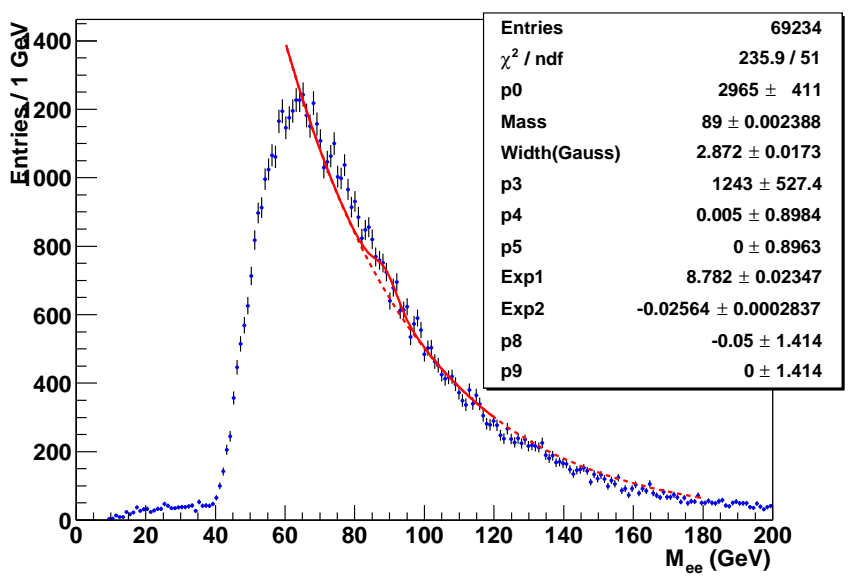

Figure 99. Di-electron object (inverted H-matrix 7 cuts) invariant mass distribution for events for the $Z$ inclusive sample in the QCD enhanced sample. The fit is dominated by the exponential background with a convoluted Gaussian and Breit-Wigner fit in the absence of a $Z$ boson signature.

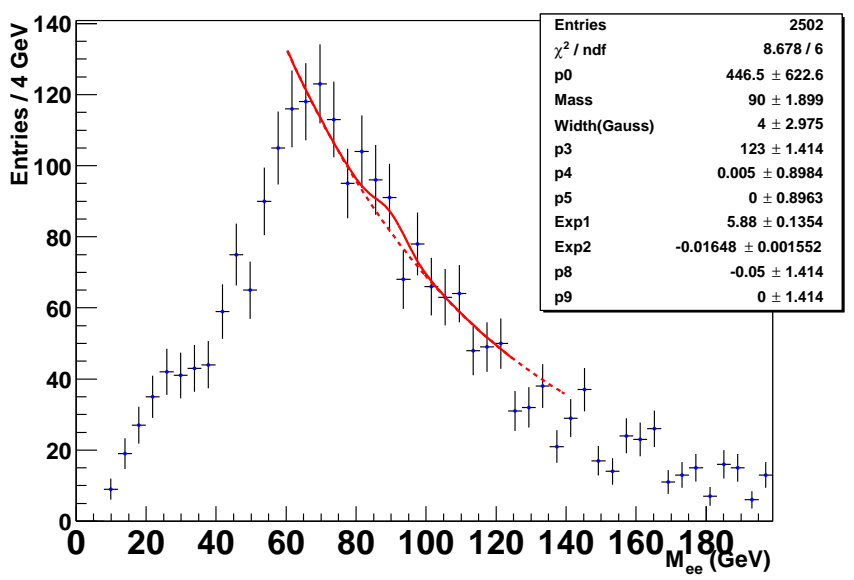

Figure 100. Di-electron object (inverted H-matrix 7 cuts) invariant mass distribution for 2 or more jets in the QCD enhanced sample. The fit is dominated by the exponential background with a convoluted Gaussian and Breit-Wigner trying to fit in the absence of a $Z$ boson signature. 


\subsection{Data and MC Comparisons}

A series of data to MC comparison plots are made at the $Z+\geq 2$ jets selection cut level. A Higgs boson mass of $115 \mathrm{GeV}$ is chosen as the signal in comparison plots. Electron distributions are shown in Figure 101. $Z$ boson distributions are shown in Figure 102. Jet distributions are shown in Figure 103 through Figure 106. Table XX compares the number of events in data to the number of events expected from the background processes and Higgs signal. These

comparisons show that the data distributions are described well with the MC simulations of the SM processes. 

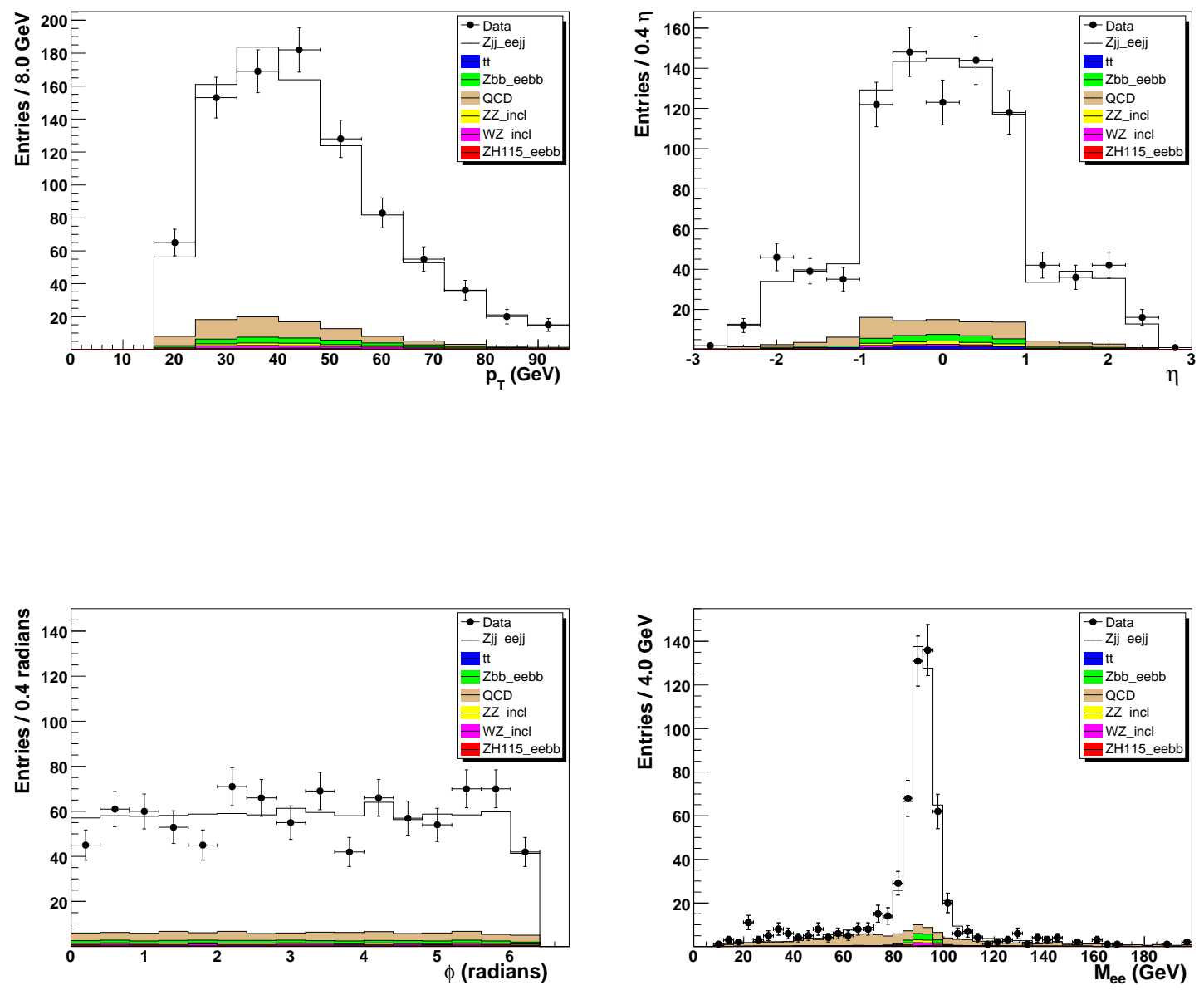

Figure 101. Kinematic distributions of the two electrons in the $Z+\geq 2$ jet sample: top left) $p_{T}$, top right) $\eta$, bottom left) $\phi$, bottom right) invariant mass. The simulation is normalized to the integrated luminosity of the data sample using the expected cross sections (absolute normalization). The $Z j j$ simulation is normalized so that the total number of simulated events equals the number of $Z+\geq 2$ jet events in data. 

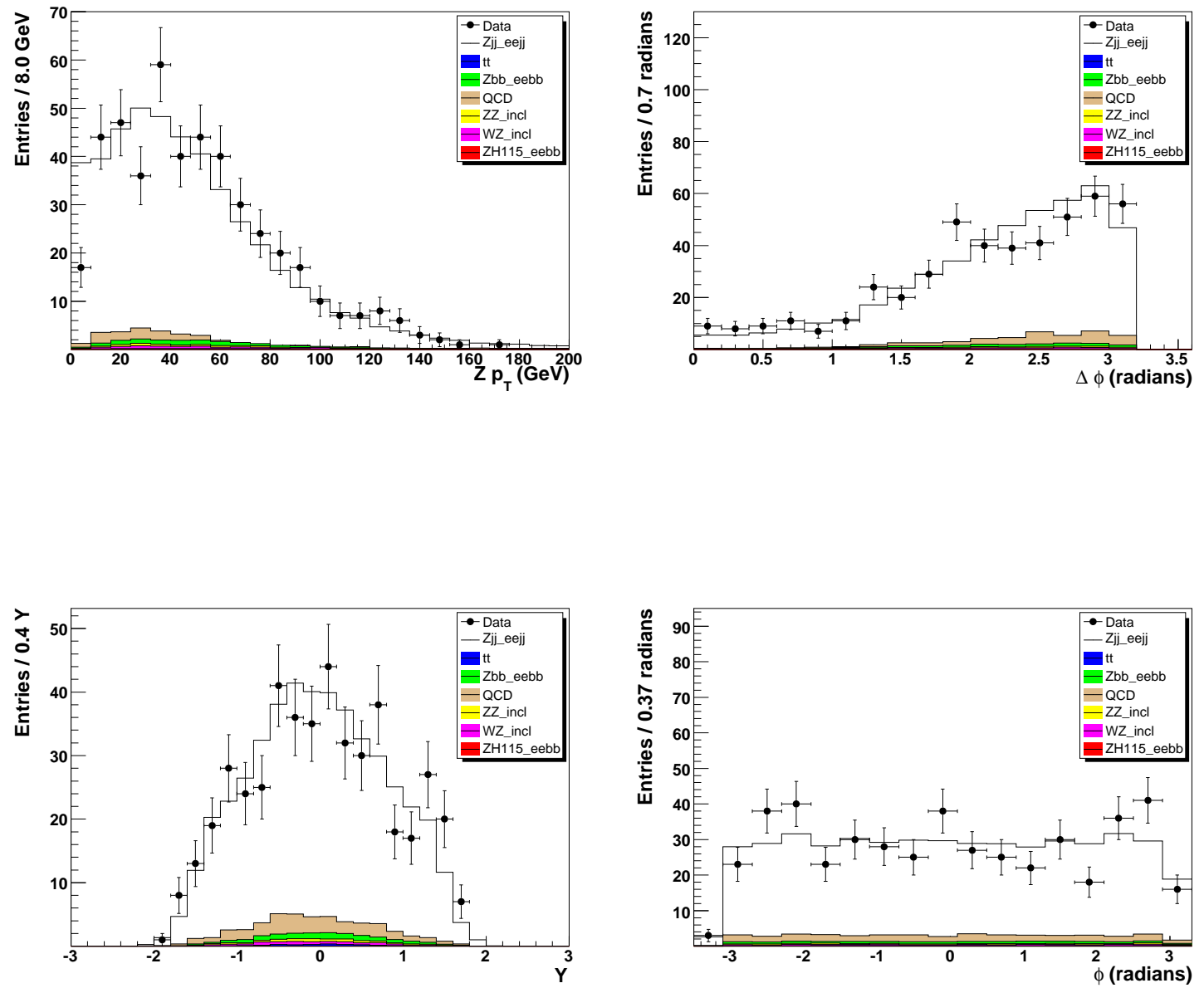

Figure 102. Kinematic distributions of the $Z$ boson in the $Z+\geq 2$ jet sample: top left) $p_{T}$, top right) $\Delta \phi$ of the 2 electrons, bottom left) Rapidity, bottom right) $\phi$. The simulation is normalized to the integrated luminosity of the data sample using the expected cross sections

(absolute normalization). The $Z j j$ simulation is normalized so that the total number of simulated events equals the number of $Z+\geq 2$ jet events in data. 

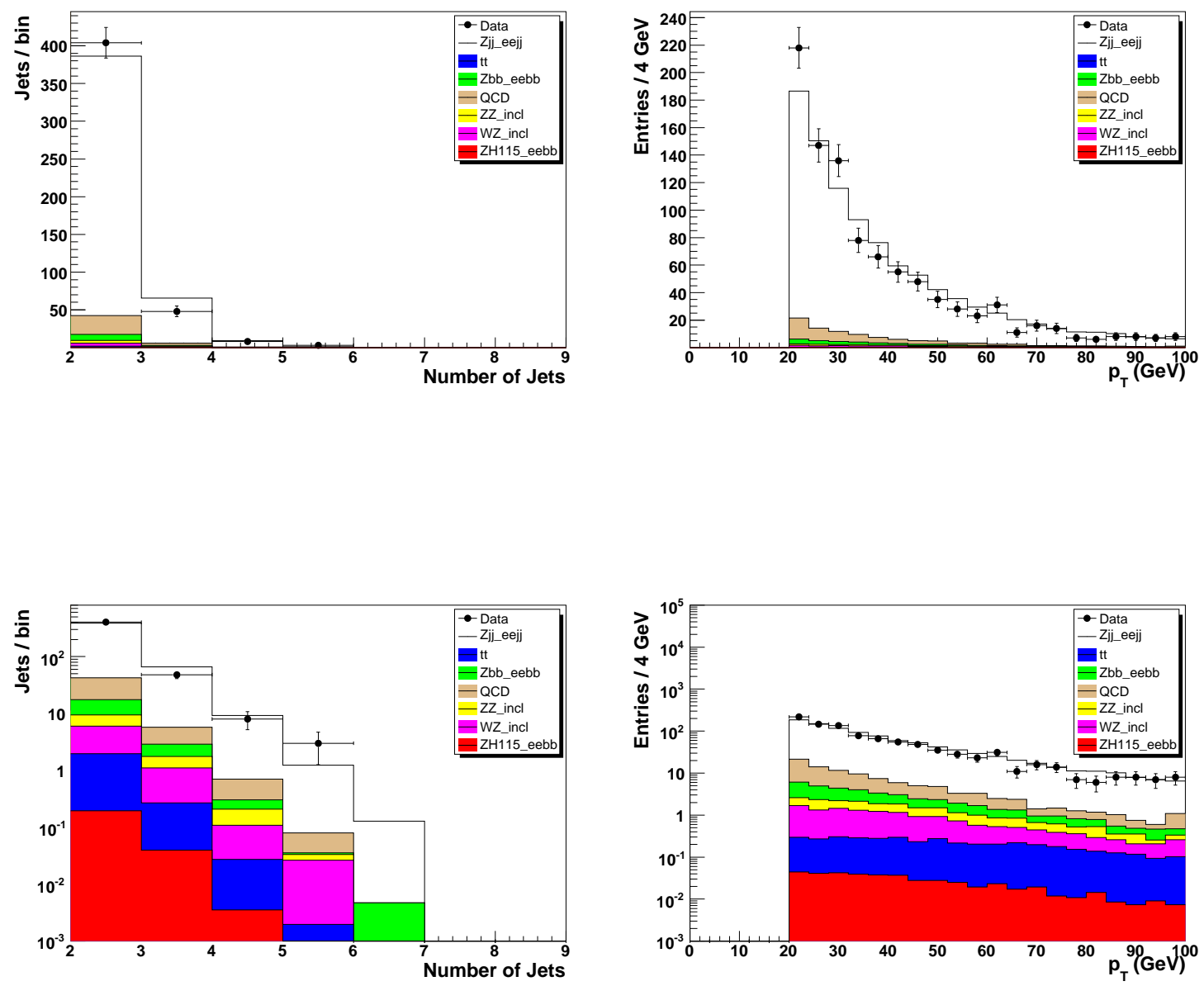

Figure 103. Distributions of jets in the $Z+\geq 2$ jet sample: top left) number of jets, top right) $p_{T}$, bottom left) number of jets (log scale), bottom right) $p_{T}$ of the jets (log scale). The simulation is normalized to the integrated luminosity of the data sample using the expected cross sections (absolute normalization). The $Z j j$ simulation is normalized so that the total number of simulated events equals the number of $Z+\geq 2$ jet events in data. 

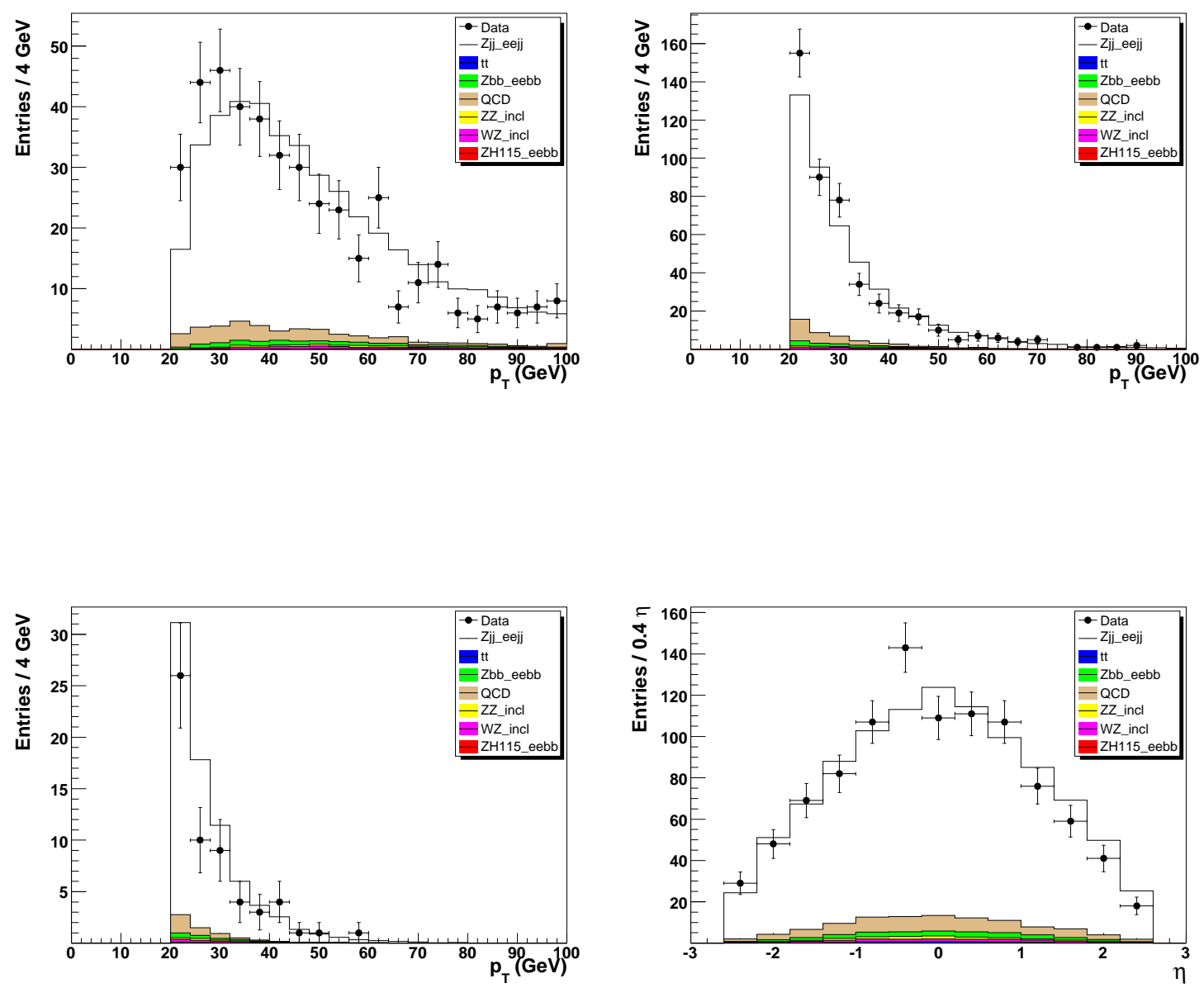

Figure 104. Distributions of jets in the $Z+\geq 2$ jet sample: top left) $p_{T}$ of leading jet, top right) $p_{T}$ of the second jet, bottom left) $p_{T}$ of the third jet (if applicable), bottom right) $\eta$ of all the jets. The simulation is normalized to the integrated luminosity of the data sample using the expected cross sections (absolute normalization). The $Z j j$ simulation is normalized so that the total number of simulated events equals the number of $Z+\geq 2$ jet events in data. 

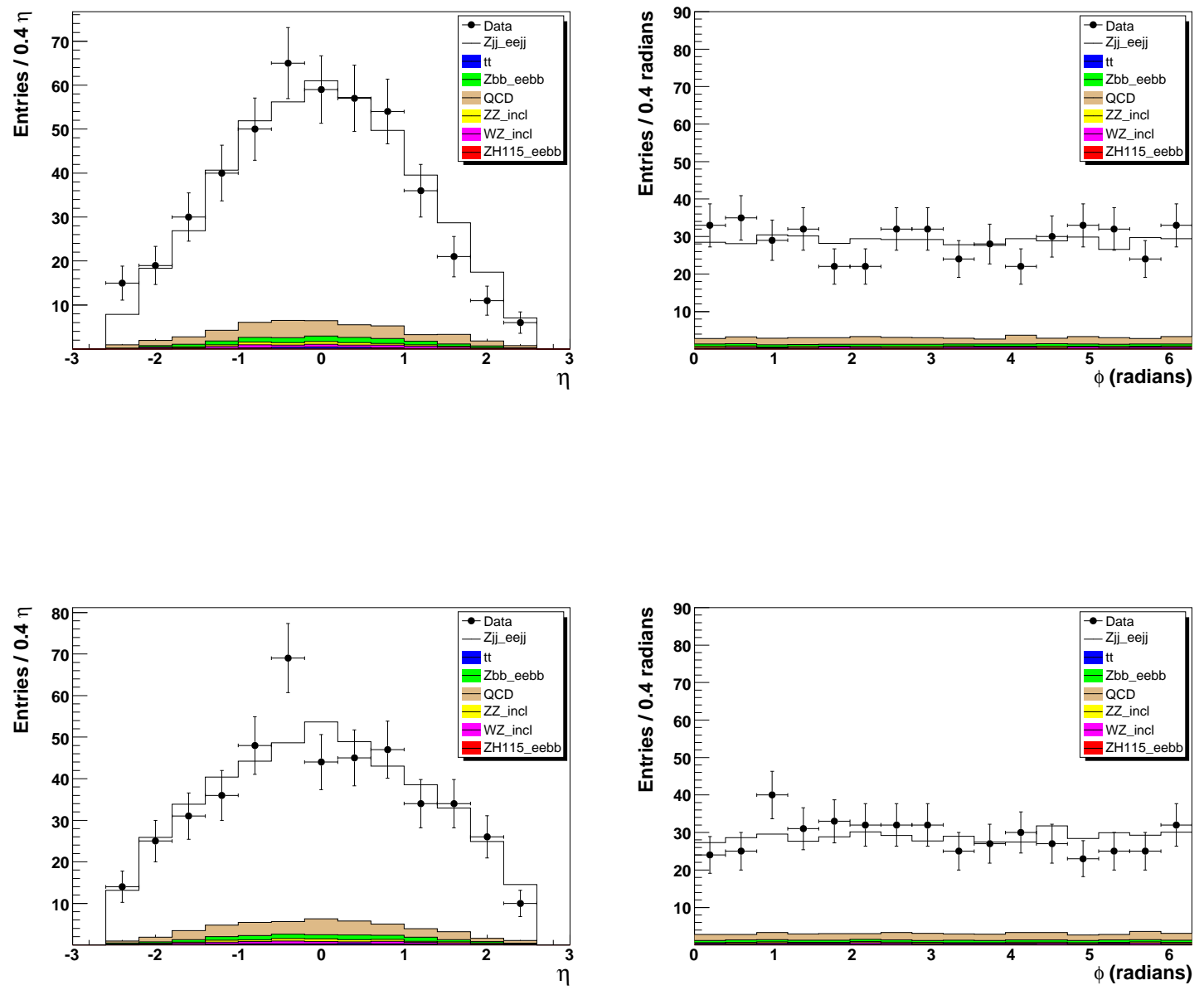

Figure 105. Distributions of the two leading jets (in $p_{T}$ ) in the $Z+\geq 2$ jet sample: top left) leading jet $\eta$, top right) leading jet $\phi$, bottom left) second jet $\eta$, bottom right) second jet $\phi$.

The simulation is normalized to the integrated luminosity of the data sample using the expected cross sections (absolute normalization). The $Z j j$ simulation is normalized so that the total number of simulated events equals the number of $Z+\geq 2$ jet events in data. 

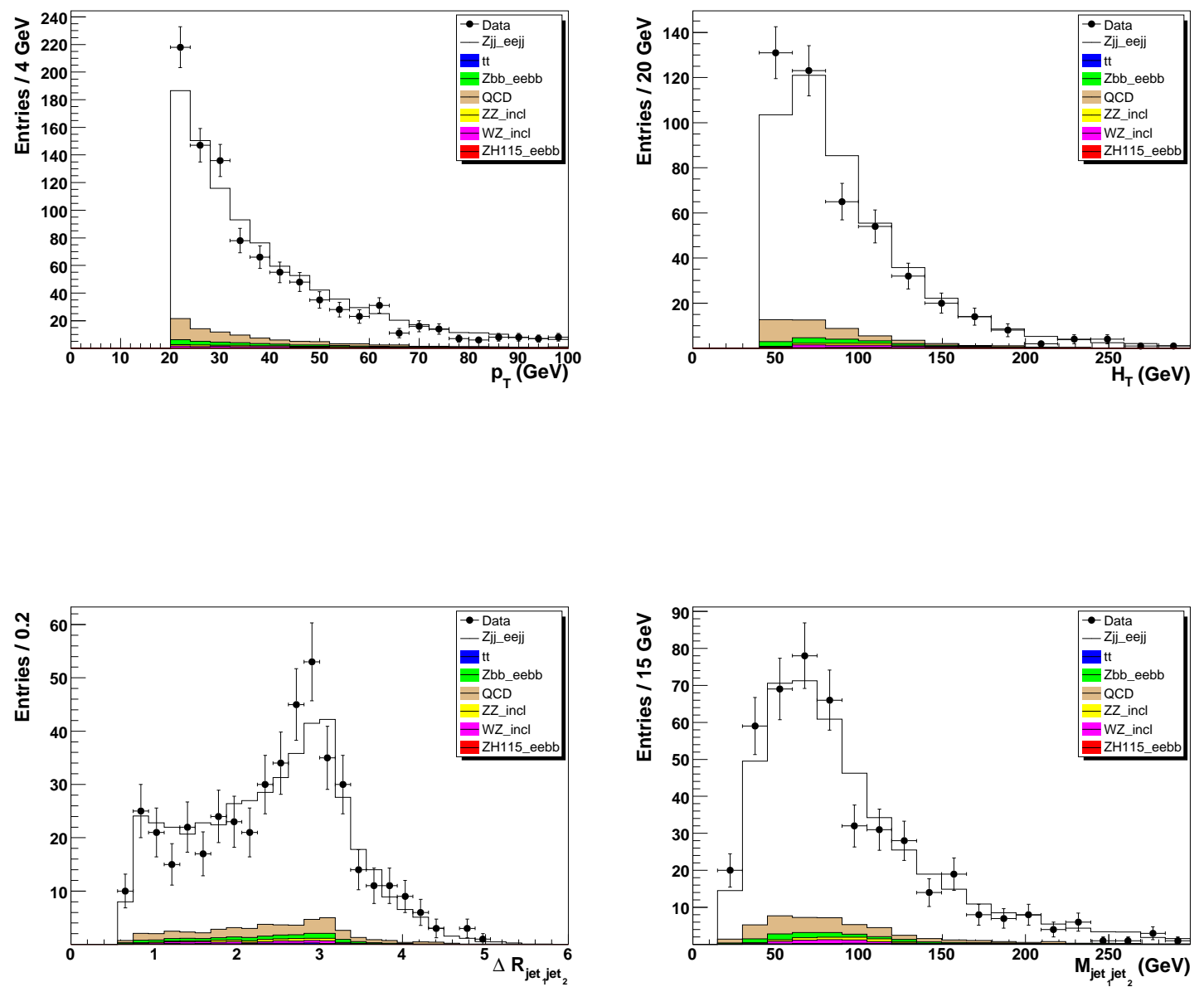

Figure 106. Distributions of jets in the $Z+\geq 2$ jet sample: top left) $p_{T}$, top right) $H_{T}$, bottom left) $\Delta \mathrm{R}$ between the two leading jets, bottom right) invariant mass of the two leading jets. The simulation is normalized to the integrated luminosity of the data sample using the expected cross sections (absolute normalization). The $Z j j$ simulation is normalized so that the total number of simulated events equals the number of $Z+\geq 2$ jet events in data. 


\begin{tabular}{|c|c|c|}
\hline & $\mathrm{Z}+\geq 2$ jets & $\begin{array}{c}\mathrm{Z}+\geq 2 \\
\text { taggable }\end{array}$ \\
\hline$t \bar{t}$ & $2.7 \pm 0.5$ & $2.2 \pm 0.4$ \\
$\mathrm{WZ}$ & $5.0 \pm 0.9$ & $3.4 \pm 0.6$ \\
$\mathrm{ZZ}$ & $4.2 \pm 0.8$ & $3.0 \pm 0.6$ \\
$Z b \bar{b}$ & $9.4 \pm 2.4$ & $6.7 \pm 1.7$ \\
$\mathrm{qcd}$ & $28.0 \pm 8.5$ & $18.5 \pm 5.7$ \\
$Z j \bar{j}$ & $413.7 \pm 86.3$ & $277.4 \pm 57.9$ \\
\hline Total Exp. & $463.0 \pm 94.5$ & $311.5 \pm 63.5$ \\
Data & 463 & 317 \\
$\mathrm{ZH}_{115}$ & $0.20 \pm 0.03$ & $0.16 \pm 0.03$ \\
\hline
\end{tabular}

TABLE XX

SUMMARY OF THE DATA, SIGNAL, AND BACKGROUND PROCESSES. THE UNCERTAINTIES INCLUDE THE STATISTICAL AND SYSTEMATIC EFFECTS. 


\subsection{Higgs Mass Search Windows}

In order to perform the counting experiment, a Higgs signal mass window must be established. An important parameter in establishing the Higgs mass windows is the di-jet mass resolution. If the resolution of the Higgs mass is too large, the Higgs signal will not be discernible from the background processes. MC samples of $Z H$ events are generated with different Higgs mass assumptions. After reconstructing the events using the same analysis package used in data, a comparison is made between the reconstructed $H \rightarrow b \bar{b}$ invariant mass and the generated one. A Gaussian fit is used to extract the mean and signal of the reconstructed Higgs invariant mass. Figure 107 shows the invariant mass distributions for the MC single and double b-tag samples. Table XXI lists the mean, width, resolution values, and the search windows $( \pm 1.5 \sigma)$ for the $5 \mathrm{ZH}$ simulated samples. The $\pm 1.5 \sigma$ search window was chosen due to its large significance. The average resolution of the 5 samples is $18 \%$ and the same search window can be used for exclusive single b-tag events. The di-jet invariant mass is approximately $85 \%$ lower than the parton generated Higgs mass due to the jet energy scale losses with respect to the quark level.

After selecting the Higgs mass search windows, a study is performed to optimize the JLIP cuts used to identify b jets. A JLIP significance was constructed as the number of signal events found in the $\mathrm{ZH}$ search windows divided by the square root of the number of background events expected in the same window. The results from the significance study are shown in Table XXIII. The tightest JLIP cut of 0.001 has the largest significance for the $Z+\geq 2 j$ sample with 1 b-tag jet, and the loosest JLIP cut of 0.04 has the largest significance for the 2 b-tag jet sample. 
Therefore, the JLIP cut of 0.04 is used when requiring two b-tag jets, and if failing, a JLIP cut of 0.001 is used, requiring 1 b-tag jet.

The percentage of events remaining after each selection cut for the Higgs signal samples (i.e. the accumulative efficiency of the Higgs signal to pass the set of selection cuts) are shown in Table XXIV.

\begin{tabular}{|c|c|c|c|c|}
\hline Higgs mass $(\mathrm{GeV})$ & mean $(\mathrm{GeV})$ & width $(\mathrm{GeV})$ & width/mean (\%) & $\begin{array}{c}\text { search } \\
\text { window }\end{array}$ \\
\hline 105 & 89 & 16 & 18 & $65-113$ \\
115 & 98 & 17 & 18 & $72-125$ \\
125 & 106 & 20 & 19 & $75-136$ \\
135 & 112 & 21 & 18 & $82-143$ \\
145 & 122 & 23 & 19 & $87-156$ \\
\hline
\end{tabular}

TABLE XXI

INVARIANT MASS RESOLUTIONS OBTAINED BY A GAUSSIAN FIT. THE AVERAGE RELATIVE RESOLUTION IS FOUND TO BE 18\%. THE HIGGS SEARCH WINDOWS ARE CENTERED ON THE MEAN AND SPAN $\pm 1.5 \sigma$. 

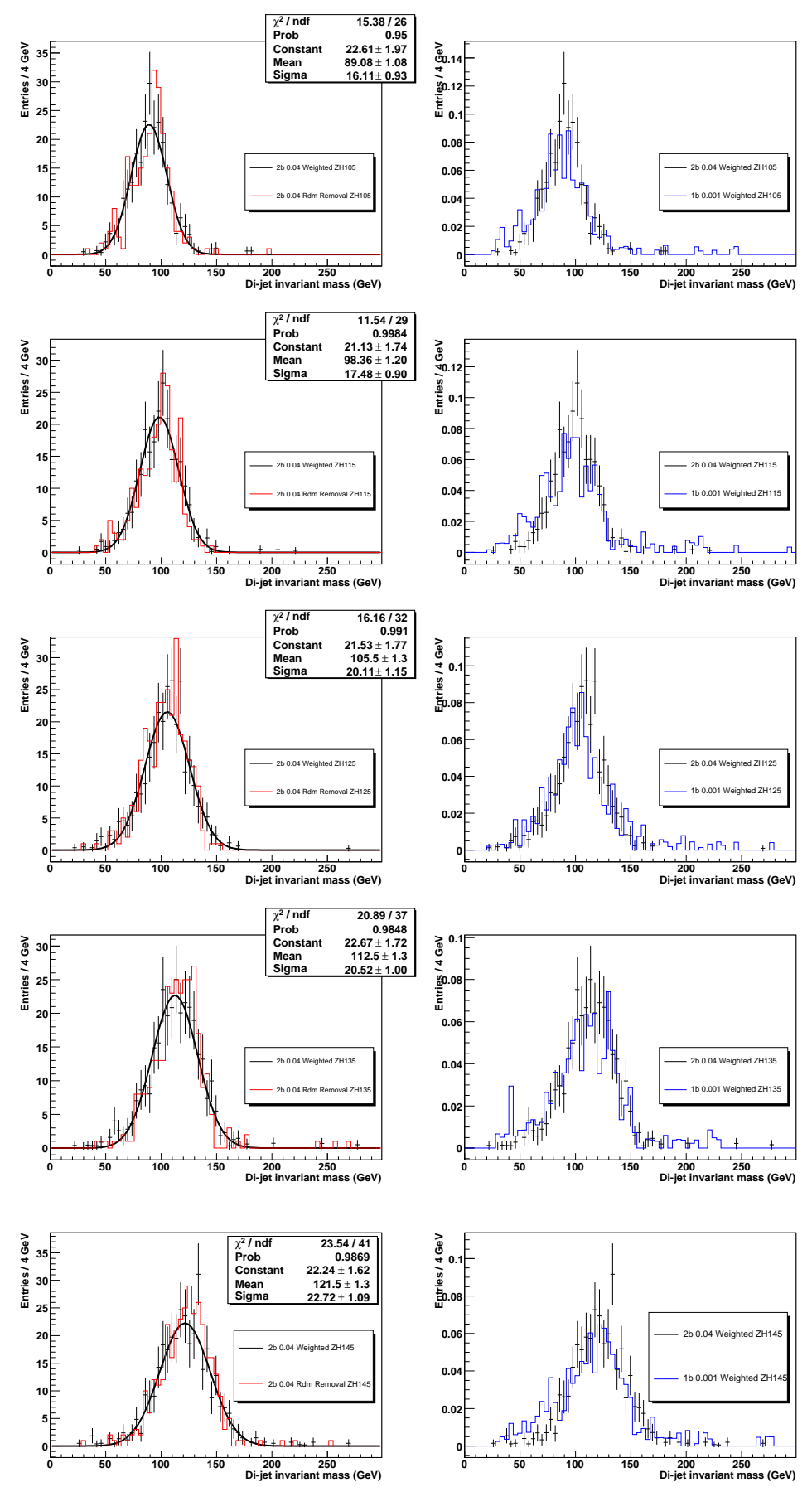

Figure 107. The resolutions of the Higgs mass given two b-tag jets. The distributions on the left have a Gaussian fit for each ZH sample. The invariant mass distributions on the right compare double b-tag jet events overlaid with exclusive single b-tag jet events. 


\begin{tabular}{|c|c|c|c|c|c|c|}
\hline JLIP cut & $\begin{array}{c}1 \mathrm{~b} \text { tag \# } \\
\text { events in } \\
\text { ZH signal }\end{array}$ & $\begin{array}{c}1 \mathrm{~b} \text { tag \# } \\
\text { events in } \\
\text { back- } \\
\text { ground }\end{array}$ & $\begin{array}{c}\text { Significance } \\
1 \mathrm{~b} \text { tag*100 }\end{array}$ & $\begin{array}{c}2 \mathrm{~b} \text { tags \# } \\
\text { events in } \\
\text { ZH signal }\end{array}$ & $\begin{array}{c}2 \text { b tags \# } \\
\text { events in } \\
\text { back- } \\
\text { ground }\end{array}$ & $\begin{array}{c}\text { Significance } \\
2 \mathrm{~b} \\
\text { tags*100 }\end{array}$ \\
\hline 0.001 & 0.070 & 8.34 & 2.39 & 0.012 & 0.88 & 1.31 \\
\hline 0.003 & 0.080 & 12.24 & 2.31 & 0.019 & 1.44 & 1.62 \\
\hline 0.005 & 0.084 & 14.70 & 2.19 & 0.023 & 1.81 & 1.72 \\
\hline 0.01 & 0.093 & 21.28 & 2.02 & 0.028 & 2.44 & 1.81 \\
\hline 0.02 & 0.100 & 31.27 & 1.78 & 0.036 & 3.19 & 2.03 \\
\hline 0.04 & 0.103 & 46.36 & 1.51 & 0.044 & 4.22 & 2.16 \\
\hline
\end{tabular}

\section{TABLE XXIII}

SIGNIFICANCE STUDY, NUMBER OF ZH EVENTS DIVIDED BY THE SQUARE ROOT OF THE NUMBER OF EVENTS FROM ALL OF THE BACKGROUND PROCESSES FOR 1 B TAG AND 2 B TAGS USING THE SIX CERTIFIED JLIP CUTS. 


\begin{tabular}{|c|c|c|c|c|c|}
\hline selection cut & $\mathrm{ZH}$ & $\mathrm{ZH}$ & $\mathrm{ZH}$ & $\mathrm{ZH}$ & $\mathrm{ZH}$ \\
& 105 & 115 & 125 & 135 & 145 \\
& $(\%)$ & $(\%)$ & $(\%)$ & $(\%)$ & $(\%)$ \\
\hline 2 electrons $p_{T}>20 \mathrm{GeV}$ & 33.3 & 33.7 & 33.8 & 32.6 & 34.4 \\
$75<M_{e e}<105 \mathrm{GeV}$ & 31.6 & 31.7 & 31.6 & 30.4 & 32.2 \\
2 jets $p_{T}>20 \mathrm{GeV}$ & 17.8 & 18.9 & 21.0 & 21.5 & 23.1 \\
2 taggable jets & 14.2 & 14.3 & 16.2 & 16.9 & 18.5 \\
exactly 1 b tag & 3.6 & 3.5 & 4.4 & 4.7 & 4.8 \\
1b $M_{j j}$ window & 2.7 & 2.5 & 3.3 & 3.5 & 3.5 \\
$\geq 2$ b tags & 5.4 & 5.1 & 6.3 & 6.6 & 7.6 \\
2b $M_{j j}$ window & 4.6 & 4.7 & 5.5 & 5.7 & 6.7 \\
\hline
\end{tabular}

TABLE XXIV

THE EFFICIENCIES OF THE FIVE MC SIGNAL SAMPLES (IN \%). 


\subsection{Results}

Table XXV shows the cumulative selection cut efficiencies for each MC process. The number of events in data and the MC simulation (with the uncertainties that are discussed in Chapter 10) for $Z+\geq 2$ jets are shown in Table XXVI.

Figure 108 shows the distributions of jet $p_{T}, H_{T}$ (the scalar $p_{T}$ sum of the two leading jets), $\Delta \mathrm{R}$ between the two leading- $p_{T}$ jets, and the invariant mass of the two leading jets with the additional requirement of exclusive single b tagging. The same four distributions, with the additional requirement of at least two b-tag jets, are shown in Figure 109. The logarithmicscale plots for these four distributions for exclusive single $b$ tag and double $b$ tag are shown in Figure 110 and Figure 111.

The final number of events in data, signal, and background for each of the five Higgs search windows are shown for exclusive single $b$ tag in Table XXVII and double b tag in Table XXVIII. No excessive of events is observed in data. Cross section upper limits are set on the SM Higgs production and discussed in the next chapter. 


\begin{tabular}{|c|c|c|c|c|c|c|c|}
\hline selection cut & $\begin{array}{c}\mathrm{ZH} \\
(\%)\end{array}$ & $\begin{array}{c}\mathrm{Zbb} \\
(\%)\end{array}$ & $\begin{array}{c}\mathrm{Zjj} \\
(\%)\end{array}$ & $\begin{array}{c}\mathrm{tt}_{2 b 2 l} \\
(\%)\end{array}$ & $\begin{array}{c}\mathrm{ZZ} \\
(\%)\end{array}$ & $\begin{array}{c}\mathrm{WZ} \\
(\%)\end{array}$ & $\begin{array}{c}\mathrm{tt}_{2 b 2 j 1 l} \\
(\%)\end{array}$ \\
\hline 2 electrons $p_{T}>20 \mathrm{GeV}$ & 33.7 & 30.8 & 28.2 & 4.1 & 2.1 & 1.1 & 0.23 \\
$75<M_{e e}<105 \mathrm{GeV}$ & 31.7 & 28.5 & 26.2 & 1.1 & 1.9 & 0.93 & 0.068 \\
2 jets $p_{T}>20 \mathrm{GeV}$ & 18.9 & 4.0 & 3.7 & 0.67 & 0.60 & 0.30 & 0.058 \\
2 taggable jets & 14.3 & 2.9 & 2.5 & 0.51 & 0.43 & 0.20 & 0.050 \\
exactly 1 b tag & 3.5 & 0.74 & 0.028 & 0.15 & 0.036 & 0.0064 & 0.015 \\
$1 \mathrm{~b} 75<M_{j j}<125 \mathrm{GeV}$ & 2.5 & 0.27 & 0.0009 & 0.055 & 0.024 & 0.0034 & 0.0061 \\
$\geq 2 \mathrm{~b}$ tags & 5.1 & 0.85 & 0.010 & 0.22 & 0.040 & 0.0025 & 0.014 \\
$2 \mathrm{~b} 75<M_{j j}<125 \mathrm{GeV}$ & 4.7 & 0.28 & 0.0004 & 0.077 & 0.022 & 0.0010 & 0.0048 \\
\hline
\end{tabular}

TABLE XXV

THE EFFICIENCIES OF THE FIVE MC SIGNAL SAMPLES AND THE MC BACKGROUNDS (IN \%). THE $Z H$ SAMPLE IS FOR A HIGGS MASS OF 115 GEV.

\begin{tabular}{|c|c|c|c|c|}
\hline & $\mathrm{Z}+\geq 2$ jets & $\begin{array}{c}\mathrm{Z}+\geq 2 \text { jets } \\
\text { taggable }\end{array}$ & $\begin{array}{c}\mathrm{Z}+1 \text { b-tag } \\
\text { exclusive }\end{array}$ & $\mathrm{Z}+2$ b-tags \\
\hline$t \bar{t}$ & $2.7 \pm 0.5$ & $2.2 \pm 0.4$ & $0.47 \pm 0.09$ & $0.83 \pm 0.16$ \\
$\mathrm{WZ}$ & $5.0 \pm 0.9$ & $3.4 \pm 0.6$ & $0.11 \pm 0.04$ & $0.04 \pm 0.02$ \\
$\mathrm{ZZ}$ & $4.2 \pm 0.8$ & $3.0 \pm 0.6$ & $0.26 \pm 0.07$ & $0.28 \pm 0.07$ \\
$Z b \bar{b}$ & $9.4 \pm 2.4$ & $6.7 \pm 1.7$ & $1.71 \pm 0.44$ & $1.97 \pm 0.51$ \\
$\mathrm{QCD}$ & $28.0 \pm 8.5$ & $18.5 \pm 5.7$ & $0.44 \pm 0.19$ & $0.18 \pm 0.10$ \\
$Z j \bar{j}$ & $413.7 \pm 86.3$ & $277.4 \pm 57.9$ & $3.09 \pm 0.73$ & $1.15 \pm 0.31$ \\
\hline Total & $463.0 \pm 94.5$ & $311.5 \pm 63.5$ & $6.07 \pm 1.31$ & $4.45 \pm 0.95$ \\
Data & 463 & 317 & 10 & 5 \\
$\mathrm{ZH}$ & $0.24 \pm 0.04$ & $0.18 \pm 0.03$ & $0.08 \pm 0.006$ & $0.065 \pm 0.011$ \\
\hline
\end{tabular}

TABLE XXVI

NUMBER OF EVENTS IN DATA WITH THE SM MC BACKGROUND PROCESSES AT FOUR DIFFERENT SELECTION CUTS. THE $Z H$ SAMPLE IS FOR A HIGGS MASS OF 115 GEV. THE $\pm 1 \sigma$ UNCERTAINTIES ARE STATISTICAL AND SYSTEMATIC. 

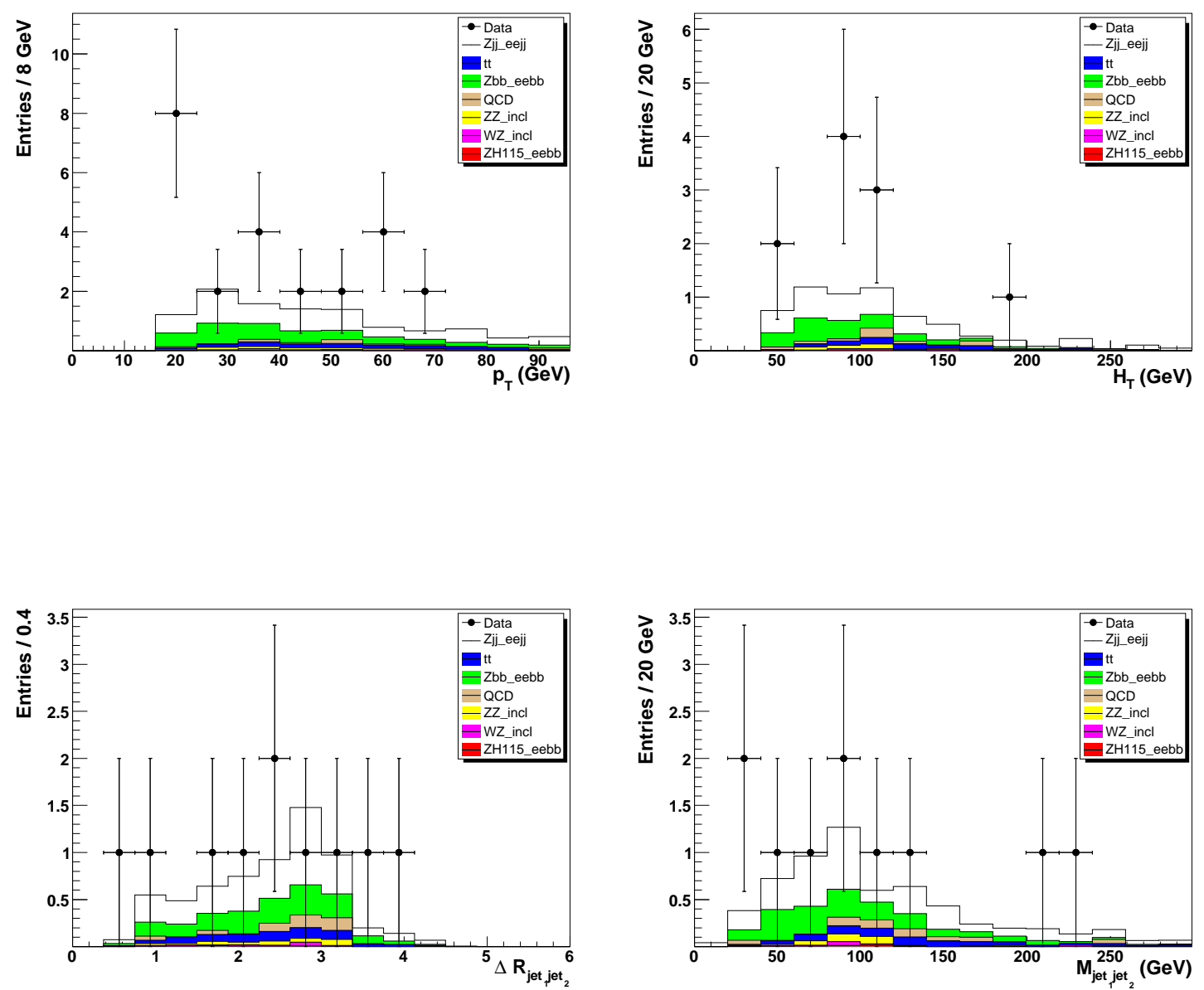

Figure 108. Distributions of jets in the $Z+\geq 2 j$ sample with 1 exclusive b-tag jet: top left) $p_{T}$, top right) $H_{T}$, bottom left) $\Delta \mathrm{R}$ between the two leading jets, bottom right) invariant mass of the two leading jets. The simulation is normalized to the integrated luminosity of the data sample using the expected cross sections (absolute normalization). The $Z j j$ simulation is normalized so that the total number of simulated events equals the number of $Z+\geq 2$ jet events in data. 

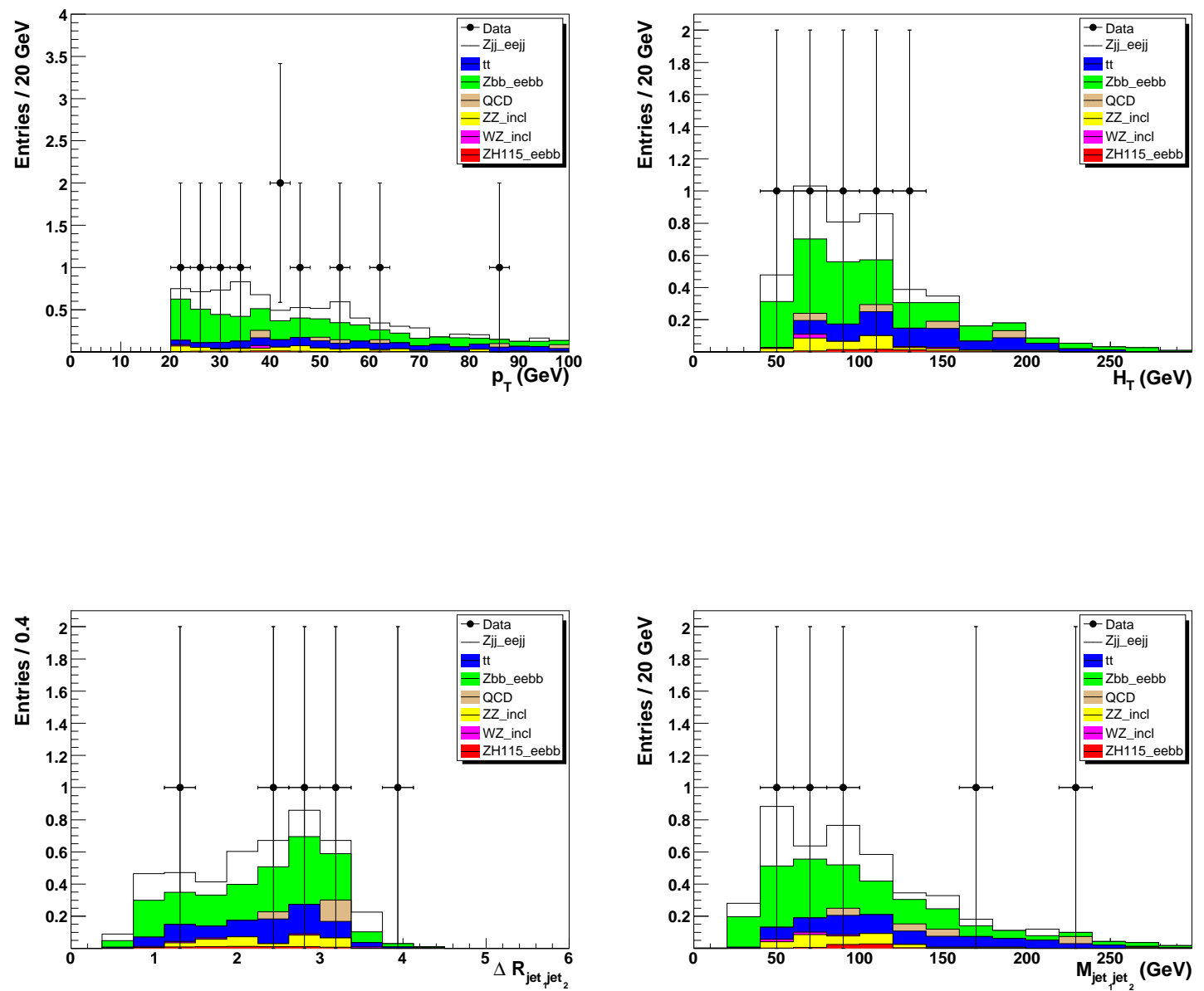

Figure 109. Distributions of jets in the $Z+\geq 2 j$ sample with at least 2 b-tag jets: top left) $p_{T}$, top right) $H_{T}$, bottom left) $\Delta \mathrm{R}$ between the two leading jets, bottom right) invariant mass of the two leading jets. The simulation is normalized to the integrated luminosity of the data sample using the expected cross sections (absolute normalization). The $Z j j$ simulation is normalized so that the total number of simulated events equals the number of $Z+\geq 2$ jet events in data. 

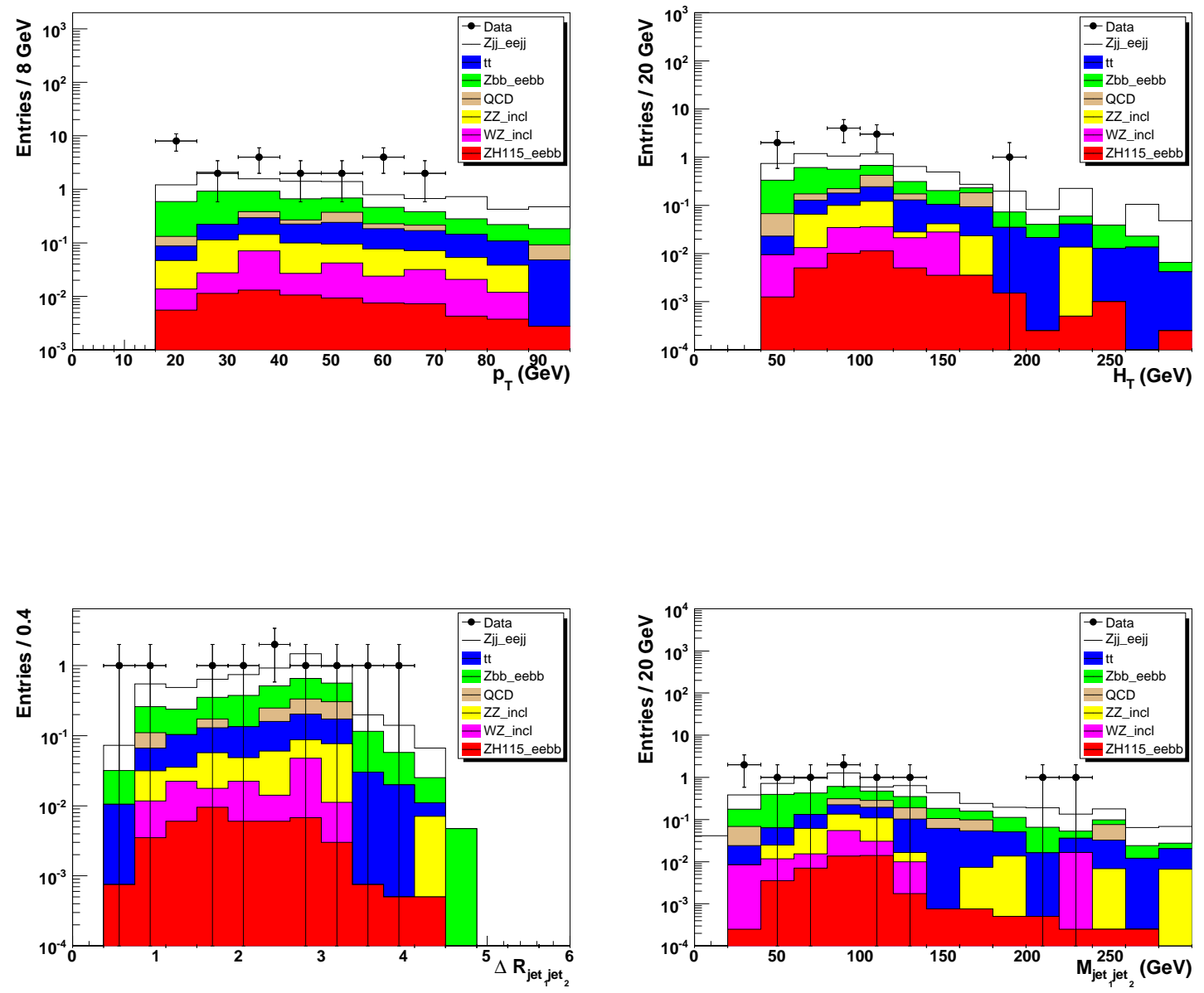

Figure 110. Distributions of jets in the $Z+\geq 2 j$ sample with exclusive $1 \mathrm{~b}$-tag jet in the $\log$ scale: top left) $p_{T}$, top right) $H_{T}$, bottom left) $\Delta$ R between the two leading jets, bottom right) invariant mass of the two leading jets. The simulation is normalized to the integrated luminosity of the data sample using the expected cross sections (absolute normalization). The $Z j j$ simulation is normalized so that the total number of simulated events equals the number of $Z+\geq 2$ jet events in data. 

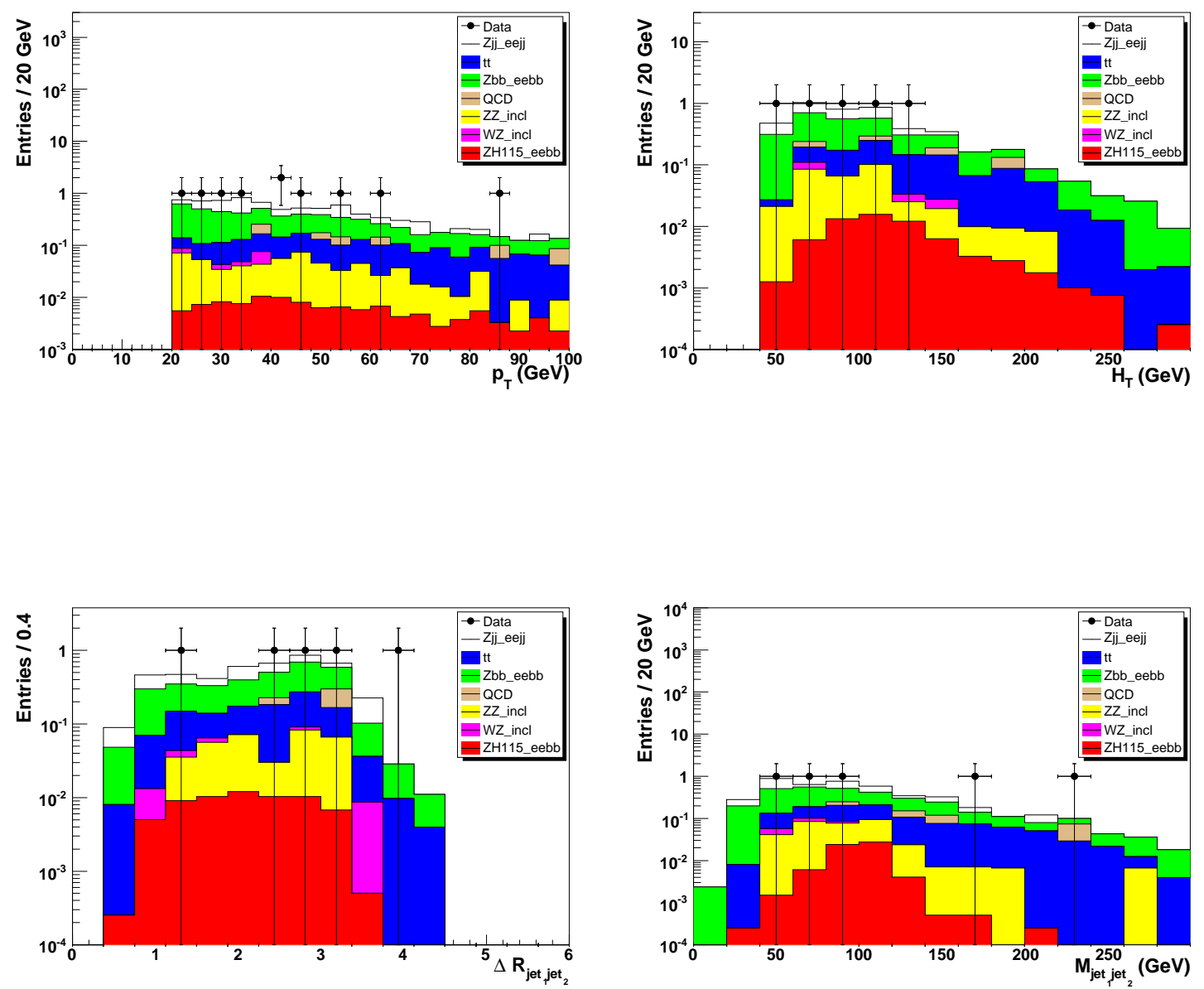

Figure 111. Distributions of jets in the $Z+\geq 2 j$ sample with at least 2 b-tag jets: top left) $p_{T}$, top right) $H_{T}$, bottom left) $\Delta \mathrm{R}$ between the two leading jets, bottom right) invariant mass of the two leading jets. The simulation is normalized to the integrated luminosity of the data sample using the expected cross sections (absolute normalization). The $Z j j$ simulation is normalized so that the total number of simulated events equals the number of $Z+\geq 2$ jet events in data. 


\begin{tabular}{|c|c|c|c|c|c|}
\hline ST & $105 \mathrm{GeV}$ & $115 \mathrm{GeV}$ & $125 \mathrm{GeV}$ & $135 \mathrm{GeV}$ & $145 \mathrm{GeV}$ \\
\hline $\mathrm{ZH}$ & $0.048 \pm 0.009$ & $0.032 \pm 0.006$ & $0.027 \pm 0.005$ & $0.017 \pm 0.003$ & $0.008 \pm 0.0013$ \\
\hline$Z b \bar{b}$ & $0.63 \pm 0.17$ & $0.64 \pm 0.17$ & $0.69 \pm 0.18$ & $0.62 \pm 0.16$ & $0.60 \pm 0.16$ \\
$Z j \bar{j}$ & $1.19 \pm 0.31$ & $0.99 \pm 0.26$ & $1.03 \pm 0.27$ & $1.11 \pm 0.29$ & $1.03 \pm 0.27$ \\
$t \bar{t}$ & $0.21 \pm 0.05$ & $0.24 \pm 0.05$ & $0.27 \pm 0.06$ & $0.26 \pm 0.06$ & $0.28 \pm 0.06$ \\
$\mathrm{ZZ}$ & $0.17 \pm 0.05$ & $0.17 \pm 0.05$ & $0.18 \pm 0.05$ & $0.14 \pm 0.04$ & $0.13 \pm 0.04$ \\
QCD & $0.18 \pm 0.08$ & $0.18 \pm 0.08$ & $0.27 \pm 0.12$ & $0.22 \pm 0.10$ & $0.26 \pm 0.12$ \\
WZ & $0.07 \pm 0.03$ & $0.06 \pm 0.03$ & $0.07 \pm 0.03$ & $0.07 \pm 0.03$ & $0.04 \pm 0.02$ \\
\hline Total & $2.45 \pm 0.54$ & $2.27 \pm 0.50$ & $2.50 \pm 0.54$ & $2.42 \pm 0.53$ & $2.34 \pm 0.51$ \\
Data & 3 & 3 & 4 & 3 & 3 \\
\hline \multicolumn{5}{|c|}{}
\end{tabular}

TABLE XXVII

NUMBER OF EVENTS WITH EXCLUSIVE SINGLE B TAGGING. EXPECTED

NUMBER OF ZH EVENTS AND BACKGROUND CONTRIBUTIONS WITHIN

$M_{H}=105,115,125,135$, AND 145 GEV SEARCH WINDOWS. THE $\pm 1 \sigma$ UNCERTAINTIES ARE STATISTICAL AND SYSTEMATIC. 


\begin{tabular}{|c|c|c|c|c|c|}
\hline DT & $105 \mathrm{GeV}$ & $115 \mathrm{GeV}$ & $125 \mathrm{GeV}$ & $135 \mathrm{GeV}$ & $145 \mathrm{GeV}$ \\
\hline ZH & $0.083 \pm 0.015$ & $0.059 \pm 0.010$ & $0.046 \pm 0.008$ & $0.027 \pm 0.004$ & $0.015 \pm 0.002$ \\
\hline Zbb & $0.68 \pm 0.18$ & $0.66 \pm 0.17$ & $0.69 \pm 0.18$ & $0.63 \pm 0.17$ & $0.64 \pm 0.17$ \\
Zjj & $0.45 \pm 0.18$ & $0.49 \pm 0.19$ & $0.49 \pm 0.19$ & $0.41 \pm 0.16$ & $0.37 \pm 0.14$ \\
tt & $0.27 \pm 0.06$ & $0.29 \pm 0.06$ & $0.31 \pm 0.07$ & $0.33 \pm 0.07$ & $0.34 \pm 0.07$ \\
ZZ & $0.15 \pm 0.04$ & $0.16 \pm 0.04$ & $0.15 \pm 0.04$ & $0.14 \pm 0.04$ & $0.13 \pm 0.03$ \\
QCD & $0.058 \pm 0.026$ & $0.044 \pm 0.026$ & $0.088 \pm 0.052$ & $0.044 \pm 0.026$ & $0.088 \pm 0.052$ \\
WZ & $0.025 \pm 0.015$ & $0.016 \pm 0.010$ & $0.016 \pm 0.010$ & $0.008 \pm 0.005$ & $0.008 \pm 0.005$ \\
\hline Total & $1.63 \pm 0.37$ & $1.66 \pm 0.38$ & $1.75 \pm 0.40$ & $1.57 \pm 0.35$ & $1.58 \pm 0.35$ \\
Data & 2 & 1 & 1 & 1 & 0 \\
\hline \multicolumn{7}{|r|}{} \\
\hline
\end{tabular}

TABLE XXVIII

NUMBER OF EVENTS WITH DOUBLE B TAGGING. EXPECTED NUMBER OF ZH EVENTS AND BACKGROUND CONTRIBUTIONS WITHIN $M_{H}=105,115,125,135$, AND 145 GEV SEARCH WINDOWS. THE $\pm 1 \sigma$ ERRORS ARE STATISTICAL AND SYSTEMATIC. 


\section{CHAPTER 10}

\section{ZH CROSS SECTION LIMITS}

This chapter describes the upper cross-section limit for the $Z\left(\rightarrow e^{+} e^{-}\right) H(\rightarrow b \bar{b})$ process. The parameters needed to set the cross-section limit are: the number of events in data, the number of background events, the integrated luminosity, and the efficiency of the Higgs signal, as well as the uncertainties for: the number of background events, the integrated luminosity, and the signal efficiency. The following describes the estimation of the uncertainties for the number of background events and signal efficiency, followed by the ZH upper cross-section limits.

\subsection{Background Uncertainties}

The source of uncertainties associated with the MC background estimates are the scale factors applied in the MC, the jet energy scale, the energy resolution of the electrons and jets, and the physics background cross sections. For all of these uncertainties, the same technique to extract the deviations in the final number of events for all MC samples is used. For example, for estimating the uncertainties from each scale factor, the scale factor is modified by $\pm 1 \sigma$ and the analysis is repeated to determine the change in the number of events remaining after all selection criteria. The uncertainties for all the MC samples are estimated for all five Higgs mass

windows. The following description of the uncertainties are for a generated Higgs mass of 115 GeV. 


\subsubsection{Scale Factor Uncertainties}

The components of statistical and systematic uncertainties for each scale factor are described in Chapter 8. The following summarizes the uncertainties from each scale factor to the expected number of events in the $115 \mathrm{GeV}$ Higgs mass search window for the two b-tag sample from the various MC samples.

\subsubsection{Trigger Efficiency Uncertainty}

The uncertainty of the electron trigger efficiency is $\pm 1.2 \%$ (Chapter 8.1 ) which results in an uncertainty of the number of events, after all selection criteria, of $\pm 1 \%$ for all MC samples.

\subsubsection{Electron RECO/ID Scale Factor Uncertainty}

The uncertainty of the scale factor is $2.0_{-0}^{+1.1} \%$ (Chapter 8.2 ) which results in an event count uncertainty of $\pm 6 \%$ for all MC samples.

\subsubsection{Track Match Scale Factor Uncertainty}

The uncertainty of the scale factor is $1.0_{-0}^{+0.8 \%}$ (Chapter 8.3 ) which results in an event count uncertainty of $\pm 1 \%$ for all MC samples.

\subsubsection{Jet RECO/ID Scale Factor Uncertainty}

The uncertainty of the scale factor (Chapter 8.4.2) which results in an event count uncertainty of $\pm 10 \%$ for $Z b \bar{b}$ and $Z j j$, and $\pm 9 \%$ for all other MC samples.

\subsubsection{Jet Taggability Scale Factor Uncertainty}

The uncertainty of the scale factor is a $2 \mathrm{D}$ parameterized average (Chapter 8.5) which results in an event count uncertainty of $\pm 4 \%$ for all MC samples. 


\subsubsection{JLIP Scale Factor Uncertainty}

The uncertainty of the scale factor (Chapter 8.6) which results in an event count uncertainty of $\pm 7 \%$ for all MC samples.

\subsubsection{Energy Uncertainty}

The energy uncertainty contains the uncertainty due to the Jet Energy Scale, the energy smearing of the electrons in MC, and the energy smearing of the jets in MC.

\subsubsection{Jet Energy Scale Uncertainty}

The jet energy scale uncertainty is parameterized as a $2 \mathrm{D}$ function of uncorrected jet $p_{T}$ and $\eta$ (Chapter 5.4.3). After propagating this uncertainty to the analysis it results in an event count uncertainty of $\pm 10 \%$ for $Z b \bar{b}$ and $Z j j$, and $\pm 7 \%$ for all other MC processes.

\subsubsection{Electron Energy Smear Uncertainty}

The uncertainty of the additional electron smearing applied to the MC (Chapter 7.2) are propagated through the analysis and produce a conservative event count uncertainty of $\pm 1 \%$ for all MC samples.

\subsubsection{Jet Energy Smear Uncertainty}

The uncertainty of the additional jet energy smearing applied to the MC (Chapter 7.2) are propagated through the analysis and produce and an event count uncertainty of $\pm 3 \%$ for $Z b \bar{b}$ and $Z j j$ and $\pm 2 \%$ for all other MC samples.

\subsubsection{Overall Experimental Uncertainties}

The overall experimental uncertainty of the number of events, added in quadrature, is $16 \%$ for $Z H_{115}$ and $18 \%$ for $Z b \bar{b}$. The $t \bar{t}, W Z$ and $Z Z$ background samples have the same uncertainty 
as $Z H_{115}$. $Z j j$ has the same uncertainity as $Z b \bar{b}$ process. A summary of the of source of the experimental uncertainty is shown in Table XXIX

\begin{tabular}{|c|c|c|}
\hline Process & $Z H_{115}$ & $Z b \bar{b}$ \\
\hline Jet energy scale & $7 \%$ & $10 \%$ \\
Jet RECO/ID scale factor & $9 \%$ & $10 \%$ \\
JLIP efficiency & $7 \%$ & $7 \%$ \\
Trigger efficiency & $1 \%$ & $1 \%$ \\
Electron RECO/ID scale factor & $6 \%$ & $6 \%$ \\
Track match scale factor uncertainty & $1 \%$ & $1 \%$ \\
Electron energy smearing & $1 \%$ & $1 \%$ \\
Jet energy smearing & $2 \%$ & $3 \%$ \\
Jet taggability scale factor & $4 \%$ & $4 \%$ \\
\hline
\end{tabular}

TABLE XXIX

A SUMMARY OF THE SOURCES OF EXPERIMENTAL UNCERTAINTY IN THE NUMBER OF EVENTS AFTER THE FINAL SELECTION CUT.

\subsubsection{Physics Background Uncertainty}

The uncertainties of the physics background processes related to the uncertainty of the MC cross sections used in this analysis are discussed in the following sections. The cross-section uncertainties are applied to the MC samples and differences in the number of events after the selection cuts, including the Higgs mass search window, are summarized in Table XXX. 


\begin{tabular}{|c|c|}
\hline Process & Uncertainty \\
\hline $\mathrm{WZ}$ & $6 \%$ \\
$\mathrm{ZZ}$ & $6 \%$ \\
$t \bar{t}$ & $8 \%$ \\
$Z b \bar{b}$ & $19 \%$ \\
$\mathrm{Zjj}$ & $7 \%$ \\
$\mathrm{ZH}_{115}$ & $7 \%$ \\
\hline
\end{tabular}

TABLE XXX

\section{MONTE CARLO CROSS SECTION UNCERTAINTIES.}

\subsubsection{1 $\frac{t \bar{t}}{}$}

The theoretical $t \bar{t}$ production cross section is estimated to be $6.8 \pm 0.53 \mathrm{pb}$ for $M_{t}=175$ $\mathrm{GeV}(77 ; 78 ; 79)$. Propagating this uncertainty to the analysis results in a change of the number of $t \bar{t}$ events of $\pm 8 \%$.

\subsubsection{2 $\underline{\mathrm{ZZ} \text { and } \mathrm{WZ}}$}

The ZZ theoretical cross section is $1.42 \pm 0.08 \mathrm{pb}(80)$, which is a $\pm 6 \%$ uncertainty. The WZ theoretical cross section is $3.68 \pm 0.25 \mathrm{pb}(80)$, which is a $7 \%$ uncertainty. Propagating these uncertainties to the analysis results in a change of the number of $\mathrm{ZZ}$ and $\mathrm{WZ}$ events by $\pm 6 \%$ and $\pm 7 \%$, respectively.

\subsubsection{3 $\underline{Z \mathbf{j j}}$}

The uncertainty of the expected number of $Z j j$ events has two components: (a) the uncertainty of the normalization process and (b) the heavy quark flavor composition of the $Z j j$ sample. 
The number of $Z j j$ events in data is estimated to be $413.7=\mathrm{N}_{\text {obs }}(463)-\mathrm{QCD}(28)-$ $\mathrm{BG}(18.6)$, where $\mathrm{N}_{o b s}$ is the total number of $Z j j$ candidate events in data, QCD represents the number of background event under the $\mathrm{Z}$ mass peak, and BG represents the number of events from background sources besides $Q C D$. The statistical uncertainty on $\mathrm{N}_{\text {obs }}$ is 21.5 events. The uncertainty on the number of QCD events is 7 events. The uncertainty on the BG contributions comes from summing the cross section uncertainties for each BG source, resulting to a total uncertainty of 2.2 events. Adding these three uncertainties in quadrature gives an uncertainty of the number of events to be $5.5 \%$ with respect to the $413.7 Z j j$ events. The second uncertainty is in terms of the heavy quark flavor composition of the $Z j j$ sample which gives a $4.3 \%$ uncertainty on the number of events after passing all selection criteria.

The combined normalization uncertainty on the number of $\mathrm{Zjj}$ events is $7 \%$, after adding the uncertainties due to normalization and the flavor composition in quadrature.

\subsubsection{4 $\underline{Z b \bar{b}}$}

The theoretical $Z b \bar{b}$ cross section is quoted as $3.74 \pm 0.45 \pm 0.12 \pm 0.15 \mathrm{pb}$ with variations from the renormalization scale, the factorization scale, and the parton distribution functions, respectively (73). The variations are added linearly and give a $19 \%$ uncertainty on the number of $Z b \bar{b}$ events passing all selection cuts.

\subsubsection{5 $\underline{Z H b \bar{b}}$}

The $Z(\rightarrow b \bar{b}) H(\rightarrow b \bar{b})(76)$ cross-section uncertainty is $7 \%$, which is the uncertainty on the number of signal event passing all selection cuts. 


\subsubsection{QCD}

The QCD background under the $Z$ mass region was estimated by using a Gaussian convoluted with Breit-Wigner for the $Z$ signal with an exponential for QCD and DY. The uncertainty on the exponential fit of the DY contribution in the $\mathrm{Zjj} \mathrm{MC}$ is $7.9 \%$. The uncertainty on the exponential fit of the DY and QCD (in data) is $16.6 \%$. Adding the two uncertainties linearly gives an overall uncertainty of $25 \%$ on the number of QCD events passing all selection cuts.

\subsubsection{Statistical Uncertainty of the MC samples}

Statistical uncertainties for the MC samples after the final selection cuts for exclusive 1 b-tagged jet and 2 b-tagged jet samples are shown in Table XXXI. The statistical uncertainty is calculated using binomial statistics. The relative statistical uncertainty is:

$$
\frac{\sigma}{\mu} ; \quad \text { where } \mu=N_{\text {initial }} \times \epsilon ; \quad \epsilon=\frac{N_{\text {final }}}{N_{\text {initial }}} ; \quad \sigma^{2}=N_{\text {initial }} \times \epsilon \times(1-\epsilon) .
$$

$N_{\text {initial }}$ is the number of MC events generated, $N_{\text {final }}$ is the number of MC events passing all selection cuts. The binomial statistical uncertainties are dominated by how efficient the MC processes are in passing all selection cuts.

\subsubsection{Overall Uncertainties}

The uncertainties of the cross sections, experimental, and statistical, are added in quadrature to estimate the final uncertainty for each sample. The uncertainties shown in Table XXXII are for the $\mathrm{ZH}_{115}$ signal process, but they are all recalculated for the four other Higgs search 


\begin{tabular}{|c|c|c|}
\hline Monte Carlo & $1 \mathrm{~b}(\%)$ & $2 \mathrm{~b}(\%)$ \\
\hline $\mathrm{ZH}_{115}$ & 8.4 & 6.5 \\
$Z b \bar{b}$ & 6.3 & 6.2 \\
$\mathrm{Zjj}$ & 16 & 33 \\
$t \bar{t}$ & 11 & 9 \\
$\mathrm{ZZ}$ & 23 & 18 \\
$\mathrm{WZ}$ & 41 & 58 \\
$\mathrm{QCD}$ & 32 & 50 \\
\hline
\end{tabular}

TABLE XXXI

THE RELATIVE STATISTICAL UNCERTAINTIES FOR THE MC SAMPLES

windows. The JES and Jet RECO/ID have smaller uncertainties as the search window is varied to higher Higgs masses, since jets forming the di-jet invariant mass will tend to be more efficient in passing the selection cuts. 


\begin{tabular}{|c|c|c|}
\hline Monte Carlo & $1 \mathrm{~b}(\%)$ & $2 \mathrm{~b}(\%)$ \\
\hline$t \bar{t}$ & 23 & 21 \\
$\mathrm{WZ}$ & 45 & 61 \\
$\mathrm{ZZ}$ & 30 & 26 \\
$Z b \bar{b}$ & 27 & 27 \\
$\mathrm{QCD}$ & 44 & 59 \\
$\mathrm{Zjj}$ & 18 & 34 \\
$\mathrm{ZH}_{115}$ & 19 & 19 \\
\hline
\end{tabular}

TABLE XXXII

OVERALL UNCERTAINTIES FOR THE SIGNAL $\left(Z H_{115}\right)$ AND THE BACKGROUND PROCESSES. 


\subsection{ZH Cross-Section Limits}

Table XXXIII contains a summary of the efficiency (the number of events passing all selection cuts divided by the number of MC generated events) for the MC signal samples used in this analysis.

Table XXXV contains a summary of the event counts for the five Higgs mass windows after the exclusive single b-tag cut has been applied. A 95\% confidence level (CL) upper crosssection limit is obtained using a Bayesian (82) approach that takes statistical and systematic uncertainties into account (see Appendix F for more details). There are 3 events in data with $2.3 \pm 0.5$ expected background events, and $0.03 \pm 0.01$ expected signal events for a Higgs boson with $115 \mathrm{GeV}$ mass. The efficiency is defined as the signal efficiency 2.5\%, for $115 \mathrm{GeV}$ Higgs, multiplied by the branching ratio of a $Z$ boson into electrons $3.4 \%$ (83). 95\% CL upper limit

of the cross section $\sigma\left(p \bar{p} \rightarrow Z H_{115}\right) \times B R\left(H_{115} \rightarrow b \bar{b}\right)$ is 17.7 pb for the exculsive single b-tag sample.

Table XXXVII shows a summary of the number of events found in each of the five Higgs mass windows for the double b-tag sample. For a Higgs mass of $115 \mathrm{GeV}$, there is 1 event in data with $1.7 \pm 0.4$ expected background events, and $0.06 \pm 0.01$ expected signal events. The signal efficiency is $0.16 \pm 0.03 \%$ which translates into a $95 \%$ CL upper limit of the cross section $\sigma\left(p \bar{p} \rightarrow Z H_{115}\right) \times B\left(H_{115} \rightarrow b \bar{b}\right)$ of 6.2 pb for the double b-tag sample.

Figure 112 compares the expected and observed 95\% CL upper limits. The expected limits are the theoretical limits given by the Bayesian approach, if the number of events found in data exactly matched the number of background events. Figure 113 summarizes the upper 
cross-section limits for the observed exclusive single b-tag and double b-tag samples. These cross-section limits are compared to the SM predictions, and the cross-section limits from the CDF experiment using a smaller data sample (Run I) and center of mass energy $(\sqrt{s}=1.8$ $\mathrm{TeV}$ ) (84). Event displays for the five double b-tagged data events are shown in Appendix G.

\begin{tabular}{|c|c|c|c|c|c|}
\hline selection cut & $\mathrm{ZH}$ & $\mathrm{ZH}$ & $\mathrm{ZH}$ & $\mathrm{ZH}$ & $\mathrm{ZH}$ \\
& 105 & 115 & 125 & 135 & 145 \\
& $(\%)$ & $(\%)$ & $(\%)$ & $(\%)$ & $(\%)$ \\
\hline $1 \mathrm{~b} M_{j j}$ window & 2.7 & 2.5 & 3.3 & 3.5 & 3.5 \\
$2 \mathrm{~b} M_{j j}$ window & 4.6 & 4.7 & 5.5 & 5.7 & 6.7 \\
\hline
\end{tabular}

TABLE XXXIII

THE EFFICIENCIES OF THE FIVE MC SIGNAL SAMPLES (IN \%). 


\begin{tabular}{|c|c|c|c|c|c|}
\hline ST & $105 \mathrm{GeV}$ & $115 \mathrm{GeV}$ & $125 \mathrm{GeV}$ & $135 \mathrm{GeV}$ & $145 \mathrm{GeV}$ \\
\hline ZH & $0.048 \pm 0.009$ & $0.032 \pm 0.006$ & $0.027 \pm 0.005$ & $0.017 \pm 0.003$ & $0.008 \pm 0.0013$ \\
\hline Zbb & $0.63 \pm 0.17$ & $0.64 \pm 0.17$ & $0.69 \pm 0.18$ & $0.62 \pm 0.16$ & $0.60 \pm 0.16$ \\
Zjj & $1.19 \pm 0.31$ & $0.99 \pm 0.26$ & $1.03 \pm 0.27$ & $1.11 \pm 0.29$ & $1.03 \pm 0.27$ \\
tt & $0.21 \pm 0.05$ & $0.24 \pm 0.05$ & $0.27 \pm 0.06$ & $0.26 \pm 0.06$ & $0.28 \pm 0.06$ \\
ZZ & $0.17 \pm 0.05$ & $0.17 \pm 0.05$ & $0.18 \pm 0.05$ & $0.14 \pm 0.04$ & $0.13 \pm 0.04$ \\
qcd & $0.18 \pm 0.08$ & $0.18 \pm 0.08$ & $0.27 \pm 0.12$ & $0.22 \pm 0.10$ & $0.26 \pm 0.12$ \\
WZ & $0.07 \pm 0.03$ & $0.06 \pm 0.03$ & $0.07 \pm 0.03$ & $0.07 \pm 0.03$ & $0.04 \pm 0.02$ \\
\hline Total Exp. & $2.45 \pm 0.54$ & $2.27 \pm 0.50$ & $2.50 \pm 0.54$ & $2.42 \pm 0.53$ & $2.34 \pm 0.51$ \\
Data & 3 & 3 & 4 & 3 & 3 \\
Efficiency(\%) & $0.090 \pm 0.017$ & $0.085 \pm 0.015$ & $0.110 \pm 0.019$ & $0.118 \pm 0.020$ & $0.118 \pm 0.020$ \\
\hline Obs limit(pb) & 16.5 & 17.4 & 15.7 & 12.2 & 12.2 \\
Exp limit(pb) & 13.3 & 14.0 & 10.5 & 9.86 & 9.86 \\
\hline
\end{tabular}

TABLE XXXV

SUMMARY OF THE ZH ANALYSIS WITH EXCLUSIVE SINGLE B TAGGING. EXPECTED NUMBER OF ZH EVENTS AND BACKGROUND CONTRIBUTIONS FOR $M_{H}=105,115,125,135$, AND 145 GEV, ALONG WITH 95\% CL CROSS-SECTION UPPER LIMITS. 


\begin{tabular}{|c|c|c|c|c|c|}
\hline DT & $105 \mathrm{GeV}$ & $115 \mathrm{GeV}$ & $125 \mathrm{GeV}$ & $135 \mathrm{GeV}$ & $145 \mathrm{GeV}$ \\
\hline ZH & $0.083 \pm 0.015$ & $0.059 \pm 0.010$ & $0.046 \pm 0.008$ & $0.027 \pm 0.004$ & $0.015 \pm 0.002$ \\
\hline Zbb & $0.68 \pm 0.18$ & $0.66 \pm 0.17$ & $0.69 \pm 0.18$ & $0.63 \pm 0.17$ & $0.64 \pm 0.17$ \\
Zjj & $0.45 \pm 0.18$ & $0.49 \pm 0.19$ & $0.49 \pm 0.19$ & $0.41 \pm 0.16$ & $0.37 \pm 0.14$ \\
tt & $0.27 \pm 0.06$ & $0.29 \pm 0.06$ & $0.31 \pm 0.07$ & $0.33 \pm 0.07$ & $0.34 \pm 0.07$ \\
ZZ & $0.15 \pm 0.04$ & $0.16 \pm 0.04$ & $0.15 \pm 0.04$ & $0.14 \pm 0.04$ & $0.13 \pm 0.03$ \\
qcd & $0.058 \pm 0.026$ & $0.044 \pm 0.026$ & $0.088 \pm 0.052$ & $0.044 \pm 0.026$ & $0.088 \pm 0.052$ \\
WZ & $0.025 \pm 0.015$ & $0.016 \pm 0.010$ & $0.016 \pm 0.010$ & $0.008 \pm 0.005$ & $0.008 \pm 0.005$ \\
\hline Total Exp. & $1.63 \pm 0.37$ & $1.66 \pm 0.38$ & $1.75 \pm 0.40$ & $1.57 \pm 0.35$ & $1.58 \pm 0.35$ \\
Data & 2 & 1 & 1 & 1 & 0 \\
Efficiency(\%) & $0.155 \pm 0.028$ & $0.157 \pm 0.027$ & $0.186 \pm 0.031$ & $0.191 \pm 0.031$ & $0.227 \pm 0.035$ \\
\hline Obs Limit(pb) & 8.2 & 6.2 & 5.1 & 5.0 & 3.2 \\
Exp Limit(pb) & 6.3 & 6.2 & 5.1 & 5.0 & 4.2 \\
\hline
\end{tabular}

TABLE XXXVII

SUMMARY OF THE ZH ANALYSIS WITH DOUBLE B TAGGING. EXPECTED NUMBER OF ZH EVENTS AND BACKGROUND CONTRIBUTIONS FOR $M_{H}=105,115,125,135$, AND 145 GEV, ALONG WITH 95\% CL CROSS-SECTION UPPER LIMITS. 


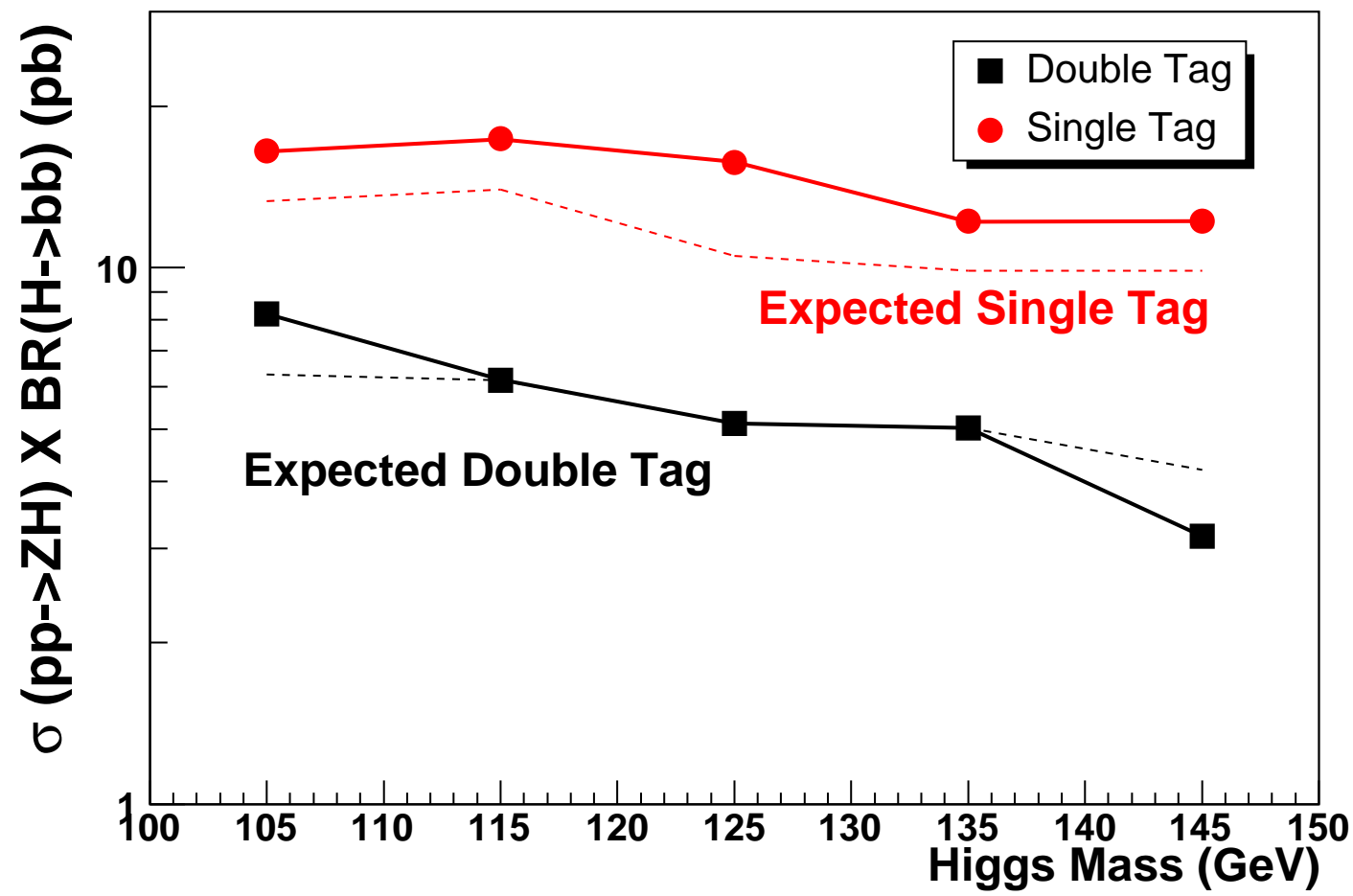

Figure 112. 95\% CL cross-section upper limit for exclusive single b-tag and double b-tag samples. The observed and expected confidence levels are compared. 


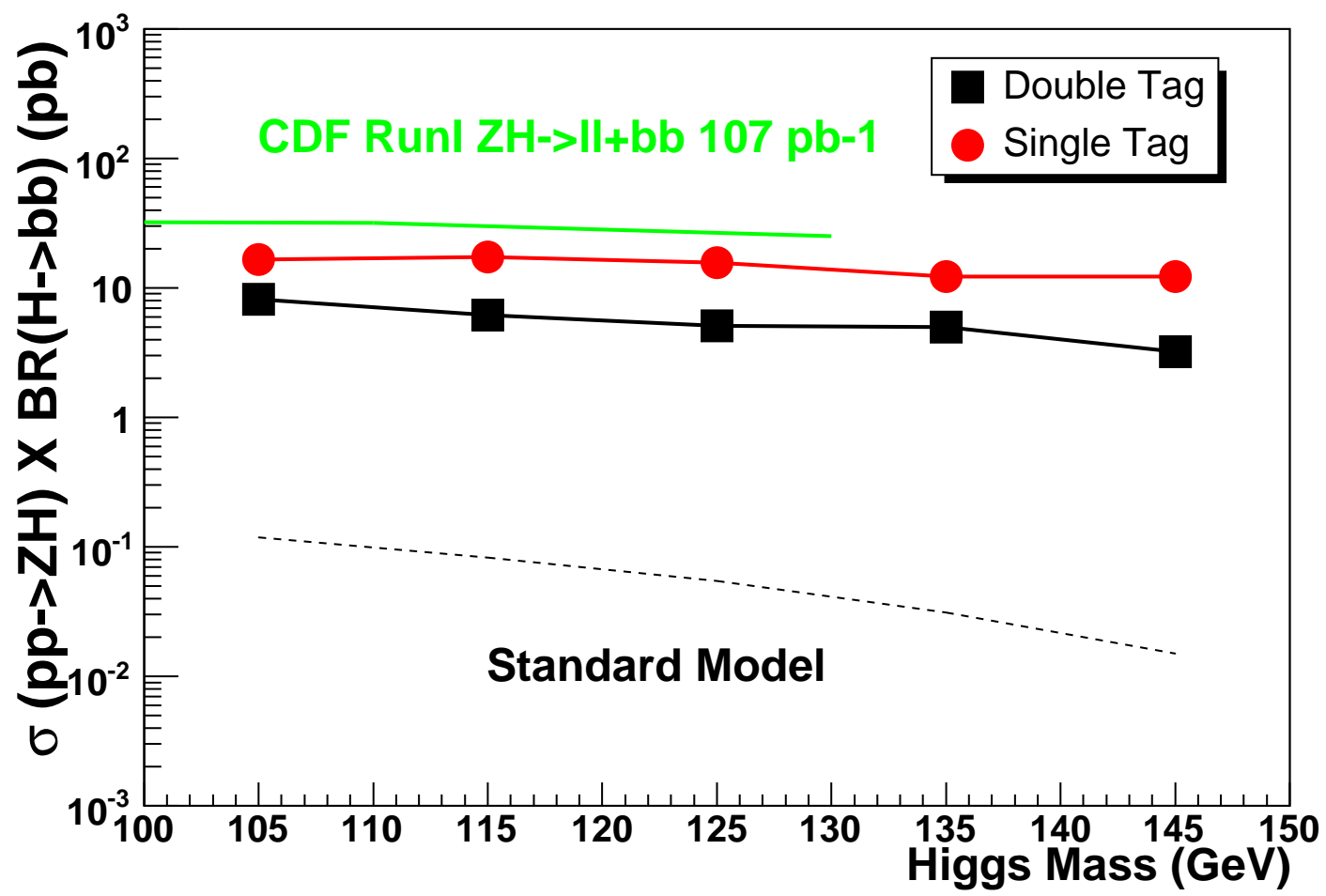

Figure 113. The observed 95\% CL cross-section upper limit for exclusive single b-tag and double b-tag samples. These cross-section limits are compared to standard model expectations and a CDF Run I combined lepton result. 


\section{CHAPTER 11}

\section{CONCLUSIONS}

A search for the SM Higgs boson in the channel $Z H \rightarrow e^{+} e^{-} b \bar{b}$ using $452 \mathrm{pb}^{-1}$ of data collected between April 2002 and August 2004 has been performed. Ten events with a $Z$ boson and one jet tagged as initiated by a b quark were found in data compared to a SM expectation number of $6 \pm 1$ events. The exclusive single b-tag production rate is consistent with the simulated expectation, and the kinematic distributions for these events are reasonably described by the simulation.

Five events with a $Z$ boson and two jets tagged as initiated by a b quark were found in data compared to a SM expectation number of $4 \pm 1$ events. The double b-tag production rate is consistent with the simulated expectation, and the kinematic distributions for these events are reasonably described by the simulation.

The $95 \%$ CL upper limit on the Higgs boson cross section, $\sigma(p \bar{p} \rightarrow \mathrm{ZH}) \times \mathrm{B}(\mathrm{H} \rightarrow b \bar{b})$, in which the two b jets are required to have $p_{T}>20 \mathrm{GeV},|\eta|<2.5$, and a di-jet mass window of $\pm 1.5 \sigma \mathrm{GeV}$ around the reconstructed Higgs mass is between 3.2 and $8.2 \mathrm{pb}$ (double tagged) and 12.2 to $17.4 \mathrm{pb}$ (exclusive single tagged) for Higgs masses between 105 and $145 \mathrm{GeV}$.

This Higgs search in the $Z H \rightarrow e^{+} e^{-} b \bar{b}$ channel was combined with a separate search in the $Z H \rightarrow \mu^{+} \mu^{-} b \bar{b}$ channel. The combined cross-section limits divided by the SM predictions are shown in Figure 114 (85). Cross-section limits from other searches are also included in this figure. Only by combining all of the decay channels, including the results from the CDF 
experiment, gathering around $8 \mathrm{fb}^{-1}$ of data per experiment, will the Tevatron be able to either show some evidence for a light mass SM Higgs boson $\left(M_{H}<135 \mathrm{GeV}\right)$ or rule out its existence up to mass of $180 \mathrm{GeV}$.

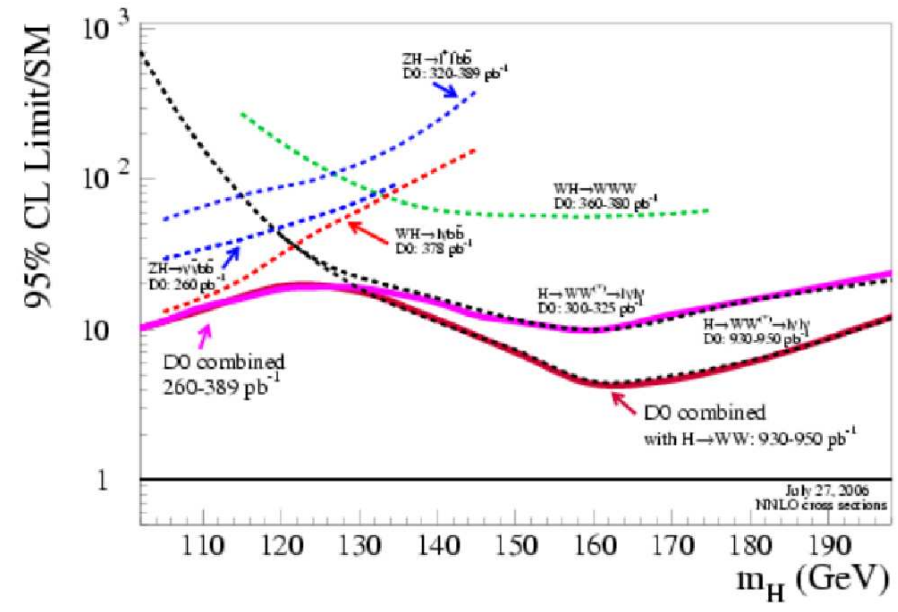

Figure 114. Upper cross section exclusion limits divided by the SM Higgs cross section for a variety of Higgs masses. 
APPENDICES 


\section{Appendix A}

\section{LEVEL-2 DATA FLOW}

The flow of data from the L2 system to the L3 system for one particular preprocessor crate, L2Cal, is shown in Figure 115. The energy of the 1280 L1 trigger towers is converted into two eight bit words. One eight bit word for the EM energy and another eight bit word for the EM+Hadronic (Total) energy. The energy is digitized into $0.25 \mathrm{GeV}$ increments. Therefore, 63.75 $\mathrm{GeV}$ is the maximum transverse energy of a trigger tower. The data from the 1280 trigger towers are transmitted through ten cables, each cable holding four sets of towers in $\eta$, and for each set in $\eta, 32$ towers in $\phi$. As implemented, one cable is linked to 128 trigger towers. Each trigger tower is readout by two eight bit words, therefore each cable has 256 bytes of data per event, excluding the header and trailer. The amount of data in one event from the L1 calorimeter trigger towers is approximately 2560 bytes. The L2 system was designed with buffers to hold up to 16 events.

The ten cables received from L1 are transmitted across fiber optic cables via a Glink transmitter (86). The Glink transmitter receives data at $53 \mathrm{MHz}$ and transmits them up to $1.3 \mathrm{~GB} / \mathrm{s}$. The Glink receiver is on a VTM (VRB Transmission Module) (87), which sends the data to the FIC (Fiber Input Converter) (88) at $53 \mathrm{MHz}$. The VTM is a Fermilab manufactured board originally designed to work with a VRB (VME Readout Buffer) (89). Glink transmits the data in 25 bit chunks in the 20 bit mode. 20 bits can be used for data, but the L1 trigger towers are in 16-bit increments, with bit 16 (beginning of event) and 17 (end of event) as protocol 
L2 cal

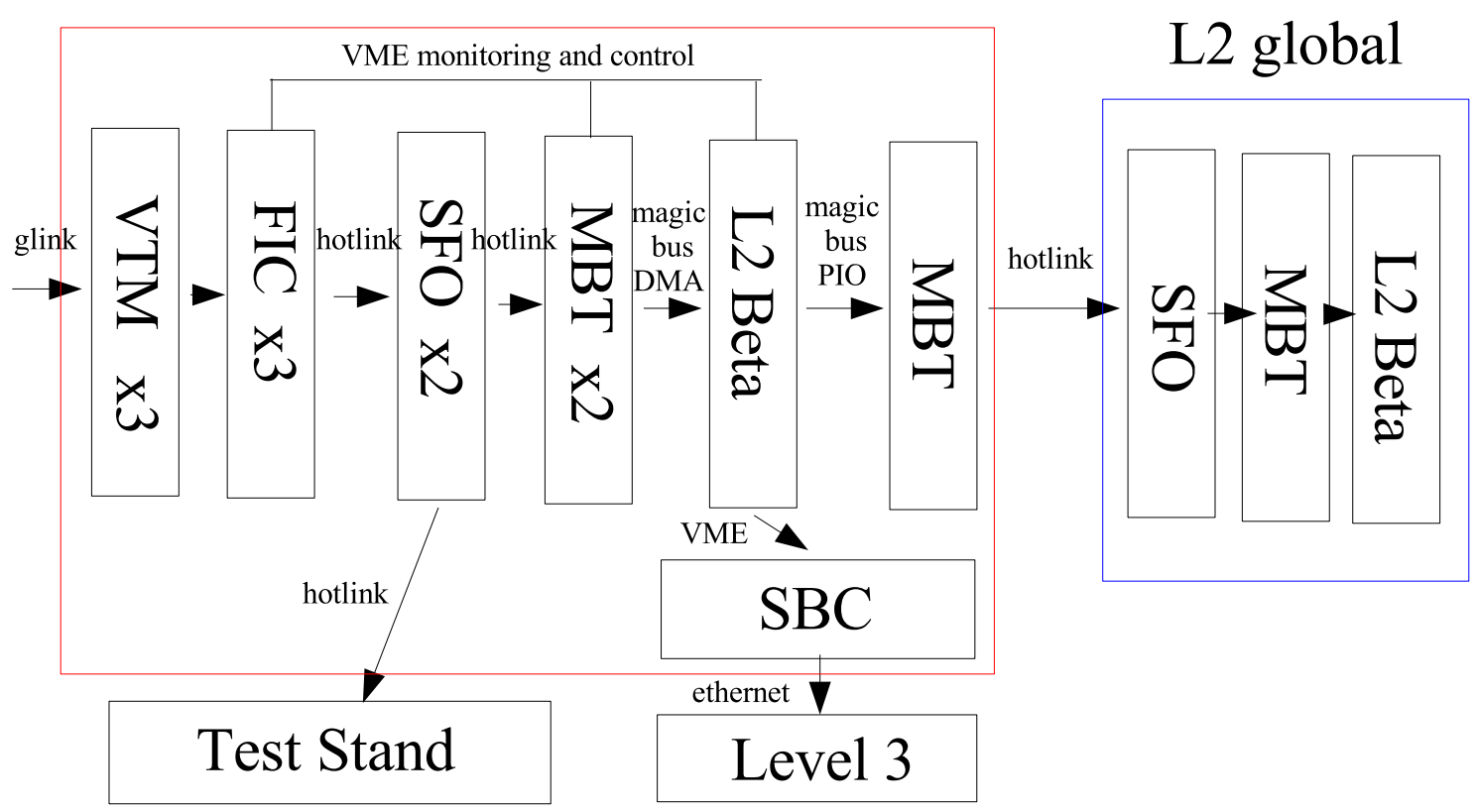

Figure 115. The flow of data from the inputs of the Level-2 calorimeter worker to the Level-3 system.

bits; 18 and 19 are unused. The Glink transmitter adds four framing bits and one flip-flop bit (alternates between 0 and 1 for each 25 bit transmission). The 16 bit words in each channel are transmitted through the VME P3 (P5/P6) (38) pins. When the FIC FIFOs (first-in-first-out) receive data, the FIC automatically starts sending the data out through the 4 differential UTP (unshielded twisted pair) Hotlink transmitters (90). Each Hotlink cable has four pairs, one pair for data and three grounded pairs. 


\section{Appendix A (Continued)}

The Hotlink data are transmitted into a Serial Fan Output (SFO) card (91), which splits the signal $1 \rightarrow 2$ without loss of differential power. One cable is used for the on-line trigger decision and the other cable is sent to a test stand. The test stand is used for algorithm and hardware debugging. Leaving the SFO, each Hotlink signal is sent to a Magic Bus Transceiver (MBT) card (92).

Each MBT has seven Hotlink input connectors, each with a set of $4-8 \mathrm{kB}$ FIFOs and two Hotlink transmitters, each with a $16 \mathrm{kB}$ FIFO. Once the MBT has received data from every channel, it starts transmitting the event, one channel at a time, until the whole event is transmitted. The data are transmitted across the "Magic Bus" (93) which is a custom built 32 bit address bus with 128 bits for data. The data can be transmitted at a rate up to 320 MB/s across the Magic Bus. Each channel on the MBT has its own Magic Bus address, which is reserved to be the lowest 6 bits of the 32 bit address space.

The L2ßeta (94) is the recipient of the data and it holds each channels' data in separate memory locations. The L2ßeta runs an algorithm which makes a list of all the EM trigger towers which have $\mathrm{E}_{T} \geq 1.0 \mathrm{GeV}$ and a separate list of Total (EM+Hadronic) trigger towers which have $\mathrm{E}_{T} \geq 2.0 \mathrm{GeV}$. Two algorithms, EM and jet, are executed to produce EM and jet trigger objects which are passed to another L2ßeta, L2GBL. The list of trigger objects is passed via Magic Bus. Next, the L2 $\beta$ eta transmits the trigger object list back to the MBT via programmed input output (PIO) (34).

PIO can transmit up to 128 bit words at a time back to the MBT. The receiver end of the PIO transmission is a FIFO chip which can hold up to 16 events. The electron and jet workers 
are limited to transmitting only 50 trigger objects each. This is done in order to avoid the overloading of L2GBL with a lot of low- $p_{T}$ trigger objects and to reduce the transmission time of PIO writes.

Next, the MBT transmits the list of trigger objects via the two Hotlink channels, one for EM and the other for jet objects. The data are transmitted into another SFO, so a copy can be sent to the L2 test stand. After the SFO the data are transmitted into another MBT in the L2GBL crate, which not only receives EM and jet objects from the L2CAL worker, but also muon, track, and preshower objects from the corresponding preprocessors.

Once the $\mathrm{MBT}$ in the $\mathrm{L} 2 \mathrm{GBL}$ crate receives data on all input channels, the data flow into L2GBL across the Magic Bus. L2GBL has up to 128 trigger decisions for each event and only considers triggers which passed the L1 trigger. The 128 bit mask trigger decision is passed via Magic Bus PIO to the MBT. The MBT transmits this bit mask to the trigger framework via the L2 hardware framework port (L2HWFW) (34).

The L2HWFW connector has 16 PECL (95) output channels and a demultiplexing card which receives 8 16-bit words and transmits the 128 bit mask to the trigger framework. When at least one of the 128 possible trigger decisions is satisfied, the serial command link (SCL) (96) broadcasts the bunch and rotation number of the accepted event to all the MBTs.

The MBTs in all the L2 crates, communicate to the L2 $\beta$ etas via PIO writes if a particular event passed the trigger requirements. Events which pass the trigger requirements have the inputs and trigger objects sent to the L3 trigger systems. If any L2 $\beta$ eta receives a L2 accept, the L2ßeta transmits the data via VME to the single board computer (SBC) (97). The SBC 
holds the event information until a L3 farm node is assigned to accept the data and the data are transmitted across 100 Mbs ethernet links to a L3 farm node.

The downloading of the trigger list, the trigger configurations, and starting, stopping, and pausing of data taking are controlled by COOR and communicated via a Windows 2000 machine called D0TCC2. D0TCC2 talks to each L2 crate via a Bit 3 card based on the VME bus. The trigger configurations are parameters passed to the algorithms. For example: the jet algorithm can be configured to use $3 \times 3$ trigger tower arrays or $5 \times 5$ trigger tower arrays when generating jet trigger objects. 


\section{Appendix B}

\section{LEVEL-2 HARDWARE: FIC, ALPHA, AND BETA BOARDS}

\section{B.0.1 FIC - Fiber Input Converter}

The FIC (Fiber Input Converter) (88) (Figure 116) was designed to convert the fiber optic Glink signals received from the L1 Calorimeter and tracking systems into Hotlink outputs transmitted via unshielded twisted pair (UTP) cables. The FIC receives data from the VTMs serially via 18 bits (2 protocol and 16 data) per channel through the J3 (p5/p6) connector which is located below the VME64x backplane. The FIC has four input channels and four Hotlink output channels.

The first word of each event received by a FIC should have the start-of-event flag (bit 16) set. A missing start-of-event bit will flag the event as having a protocol error and will be generated during a FIC monitoring cycle. The last word of an event received by a FIC should be flagged by setting the end-of-event bit (bit 17). The 16-bit words get broken down into two 8-bit words when transmitted across the Hotlink channels. The FIC appends a 16-bit word which is a 8 bit XOR longitudinal parity and sets bit 15 if there were protocol errors.

The FICs start emptying their FIFOs via Hotlink as soon as data enters. The FIFO input

clock frequency is $53 \mathrm{MHz}$ at 16-bits while the FIFO output clock frequency is $16 \mathrm{MHz}$ at 16 bits. The Hotlink transmitter receives data in increments of 8-bits (out of 10) at $16 \mathrm{MHz}$ and transmits out at $160 \mathrm{Mb} / \mathrm{s}$. 


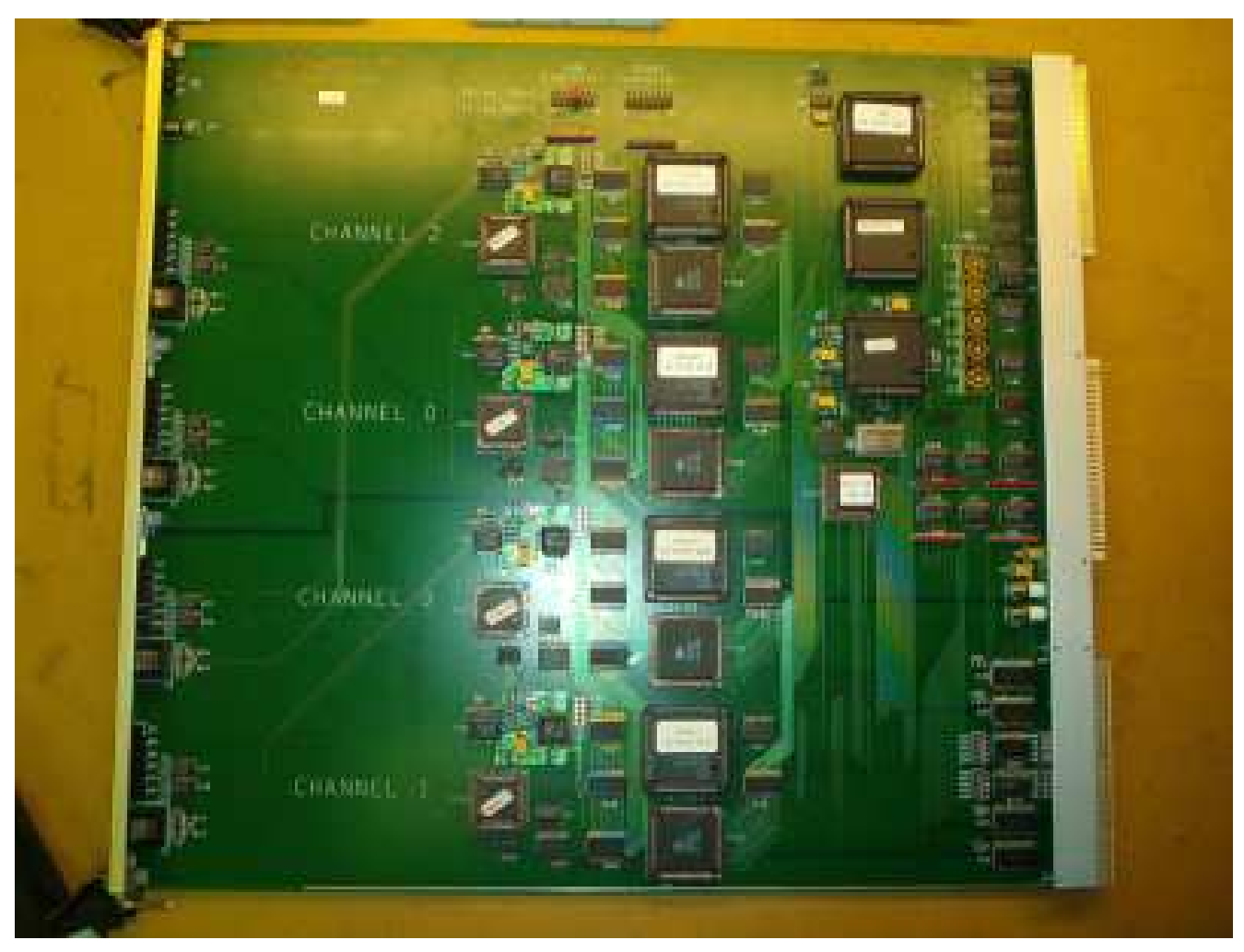

Figure 116. FIC (Fiber Input Converter).

\section{B.0.2 Level-2 Alpha}

The original core of the L2 system was implemented by $9 \mathrm{U}$ monolithic Alpha boards (Figure 117)(99). The following lists the main features of the Alpha board design:

- $500 \mathrm{MHz} 64$ bit RISC (reduced instruction set computer) Alpha $21164 \mathrm{CPU}$;

- ICache $8 \mathrm{kB}$, DCache $8 \mathrm{kB}$, and Level-2 cache 96kB;

- 21172 Core Logic Chip Set - 1 central controller (CIA) and 4 buffer chips;

- PCI 64 bit $33 \mathrm{MHz}$; 
- 128 kB SROM for initialization;

- 1 MB Flash ROM for operating system initialization;

- $128 \mathrm{MB}$ of DRAM;

- Xilinx FPGA, Cypress CPLD, and FIFOs for Magic Bus DMA. - 128 bits from Magic Bus into 64 bits across the PCI bus to memory;

- Xilinx XC4036EX and buffer chips for up to 128 bit PIO;

- 64 bit VME Tundra Universe II chip with buffers and drivers;

- Trigger Supervisor Interface FPGA;

- 264 bit PCI slots, two serial ports, parallel port;

- 1 GB IDE hard disk drive running Linux.

\section{B.0.3 Level-2 Beta}

The L2 $\beta$ eta (94), Figure 118, is an upgraded version of the L2Alpha processor used for the early part of Run IIa. The original L2Alpha board had production difficulties which limited the full implementation of the L2 trigger system as initially designed. In addition, the L2Alpha had a fixed CPU speed and as the luminosity increased, the L2 trigger system became more complex and faster CPU speed was needed.

The modular design features a commodity 6U cPCI (Compaq PCI) (100) dual $1.0 \mathrm{GHz}$ Pentium-processor single board computer. The $6 \mathrm{U}$ processor card plugs into a $9 \mathrm{U}$ custom build adapter card. The $9 \mathrm{U}$ adapter card communicates with the $6 \mathrm{U}$ PCI card via a 64 bit PCI bridge clocked at $66 \mathrm{MHz}$. The $9 \mathrm{U}$ adapter card, besides from providing power and hosting the hard 


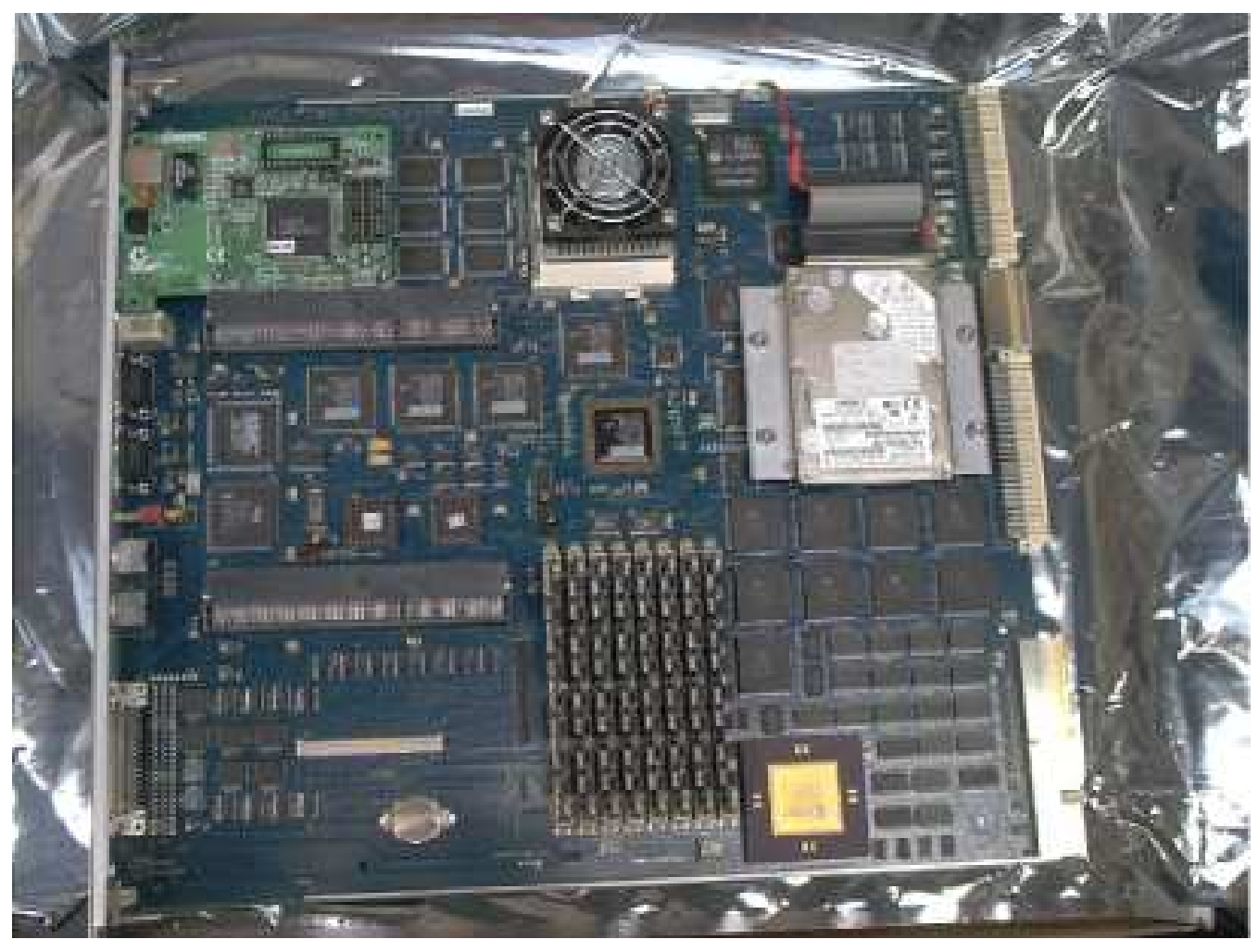

Figure 117. Level-2 alpha board.

drive, contains the VME, Magic Bus, and TSI (Trigger Supervisor Interface) (99) interface. The $6 \mathrm{U}$ cPCI board chosen was developed by Advantech (101) and has the following features:

- 2 Intel Pentium III 32 bit $1 \mathrm{GHz}$ processors;

- ICache 16kB, Dcache 16kB, and Level-2 Cache $256 \mathrm{kB}$;

- ServerWorks ServerSet III LE chipset;

- $133 \mathrm{MHz}$ Front side bus;

- 1 GB of PC-133 SDRAM with ECC; 
- 64 bit $33 \mathrm{MHz}$ CompactPCI bus;

- 2 - 10/100Base-TX ethernet ports;

- EIDE - ATA 33 up to 4 hard drives;

- 64 bit $33 \mathrm{MHz}$ PCI to PCI bridge Intel 21154;

- 2 USB 1.0 ports;

- 2 RS-232 serial ports;

- PS/2 Keyboard and mouse ports;

- running Red Hat Linux Version 9.0.

The custom build $9 \mathrm{U}$ adapter cards have the following features:

- 64 bit VME Tundra Universe II with buffer and drivers;

- Magic Bus DMA, Magic Bus PIO, and TSI hosted on one FPGA Xilinx XCV405E;

- PLX 9656 controller - 64 bit PCI to local bus (to Xilinx XCV405E) 32 bit 66MHz;

- assorted logic converters and drivers;

- hard drive connection.

The $9 \mathrm{U}$ cPCI adapter card connects directly to three separate buses. First, the $6 \mathrm{U}$ SBC is attached at one end linking the PCI bus, the other end of the card is attached to the $6 \mathrm{U}$ connectors for the VME64x bus, and a 3U connector for the 47X5 pin Magic Bus. The Xilinx FPGA arbitrates the Magic Bus DMA, which receives data from the MBT into memory; the Magic Bus PIO, which sends the trigger object list to the MBT; and the TSI functionality, 
which has some Magic Bus logic, trigger information, and hardware firmware scalers. The Xilinx FPGA has $70 \mathrm{kB}$ of RAM and 10k logic cells to act as FIFOs and address translation tables.

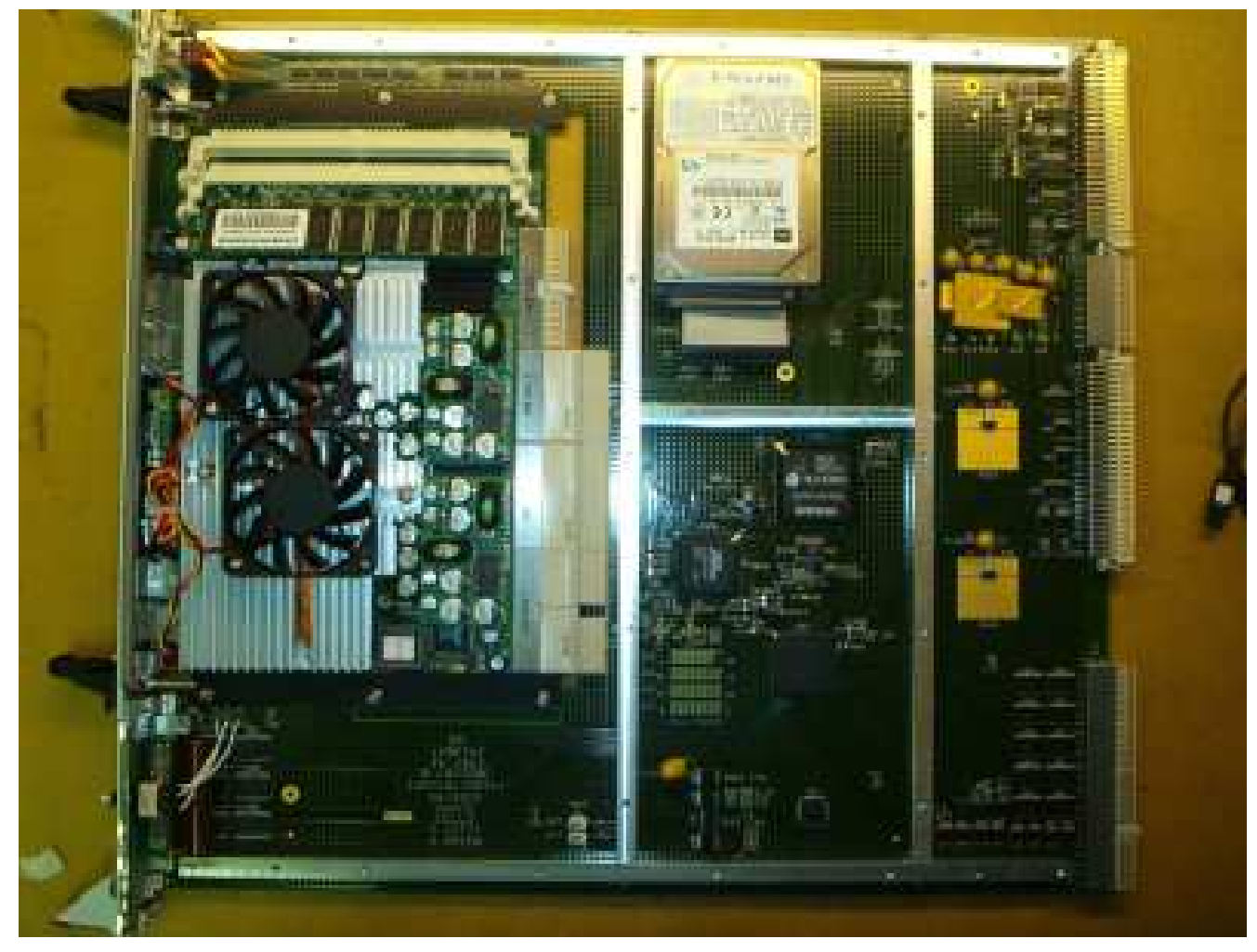

Figure 118. Level-2 beta with $6 \mathrm{U}$ SBC and $9 \mathrm{U}$ adapter card. 


\section{Appendix C}

\section{LEVEL-2 CALORIMETER ALGORITHMS}

\section{C.0.4 Level-2 EM algorithm}

The electromagnetic algorithm forms photon and electron candidate objects by clustering two EM trigger towers (TT) together. The algorithm works in the following manner (102):

- create a list of seed trigger towers which have EM energy $\geq 1.0 \mathrm{GeV}$;

- sort the seeds in descending order of $\mathrm{E}_{T}$;

- starting from the largest $\mathrm{E}_{T}$ seed, find the largest nearest neighbor $(\mathrm{NN})$ within \pm 1 trigger tower in $\eta$ or $\phi$;

- remove the nearest neighbor from the seed list (if it was in the list);

- add the energy of the cluster (seed and NN) and re-sort the clusters in descending order of $\mathrm{E}_{T}$;

- calculate the EM fraction of the cluster (Seed EM $\mathrm{E}_{T}+\mathrm{NN}$ EM $\mathrm{E}_{T}$ ) / (Seed Total $(\mathrm{EM}+$ Hadronic $) \mathrm{E}_{T}+\mathrm{NN}$ Total $\left.\mathrm{E}_{T}\right) ;$

- calculate the isolation fraction 1 - ((Seed EM $\left.\mathrm{E}_{T}+\mathrm{NN} \mathrm{EM} \mathrm{E}_{T}\right) /\left(\right.$ sum of Total $\mathrm{E}_{T}$ of $3 \times 3$ trigger towers centered on the seed trigger tower));

- calculate the energy weighted $\eta$ and $\phi$ position

$$
-\eta_{\text {weighted }}=\left(\mathrm{E}_{T} \text { Seed }^{*} \eta+\mathrm{E}_{T} \mathrm{NN}^{*} \eta\right) /\left(\mathrm{E}_{T} \text { Seed }+\mathrm{E}_{T} \mathrm{NN}\right) \text { same for } \phi
$$




\section{Appendix C (Continued)}

- set a bit if the EM $\mathrm{E}_{T}$ or Total $\mathrm{E}_{T}$ of a trigger tower related to the EM object is larger than the saturation value of $61.75 \mathrm{GeV}$;

- pass the EM candidates to L2GBL.

Table Table XXXVIII lists the parameters per EM object passed to L2GBL.

\begin{tabular}{|c|c|}
\hline variable & Bits \\
\hline$\eta$ & 8 \\
$\phi$ & 8 \\
$E_{T}$ & 16 \\
EM fract $_{\text {seed }}$ & 8 \\
Iso fract & 8 \\
$\eta_{\text {leadingTT }}$ & 8 \\
$\phi_{\text {leadingTT }}$ & 8 \\
$\eta_{\text {otherTT }}$ & 8 \\
$\phi_{\text {otherTT }}$ & 8 \\
Saturated EM tower & 1 \\
Saturated Total tower & 1 \\
no Neighbor & 1 \\
\hline
\end{tabular}

TABLE XXXVIII

EM OUTPUT TO L2GLOBAL.

Table XXXIX shows the information that is sent to L2GBL for every EM object based on trigger list $\mathrm{v} 13$. 


\begin{tabular}{|c|c|}
\hline variable & Bits \\
\hline$\eta$ & 8 \\
$\phi$ & 8 \\
$E_{T}$ & 16 \\
$E_{T}^{N N}$ & 8 \\
EMfract $_{\text {seed }}$ & 8 \\
EMfract $_{N N}$ & 8 \\
Iso fract & 8 \\
$\eta_{\text {leadingTT }}$ & 8 \\
$\phi_{\text {leadingTT }}$ & 8 \\
Saturated EM tower $_{\text {Saturated Total tower }}$ & 1 \\
$\eta$ or $\phi$ NN & 1 \\
position NN $^{2}$ & 1 \\
\hline
\end{tabular}

TABLE XXXIX

\section{EM OUTPUT TO L2GLOBAL FOR TRIGGER LISTS GREATER THAN V12.}

\section{C.0.5 Level-2 jet algorithm}

The jet algorithm forms jet candidate objects by clustering the total transverse energy of trigger towers in a $5 \times 5$ array $(\Delta \eta \times \Delta \phi)$. The algorithm is outlined as follows: (102):

- use the L1 bit mask to create a list of seed trigger towers with total $\mathrm{E}_{T} \geq 2.0 \mathrm{GeV}$;

- calculate cluster total $\mathrm{E}_{T}$ using 5x5 trigger tower arrays centered around the seeds;

- sort the list of jet objects in descending order of $\mathrm{E}_{T}$;

- when two jets overlap, remove the lower $\mathrm{E}_{T}$ jet if:

- $\Delta p h i$ or $\Delta e t a$ between the centers of two adjacent clusters is less than four trigger towers; 


\section{Appendix C (Continued)}

- if the keep_row variable is set to zero, $\Delta p h i$ and $\Delta e t a$ between the centers of two adjacent clusters is less than four trigger towers;

- if the keep_row and keep_corner variable is set to zero, $\Delta p h i$ or $\Delta e t a$ between the centers of two adjacent clusters is less than five trigger towers;

- calculate the energy weighted center $\eta$ and $\phi$ in units of 0 to 159 :

$$
-\eta_{\text {weighted }}=\frac{\sum_{i} E_{T_{i}} \eta_{i}}{\sum_{i} E_{T_{i}}} \quad \phi_{c m}=\frac{\sum_{i} E_{T_{i}} \phi_{i}}{\sum_{i} E_{T_{i}}}
$$

- evaluate the $\eta$ and $\phi$ position of the lead trigger tower;

- set a bit if the total $\mathrm{E}_{T}$ of a trigger tower associated with the jet object is larger than the saturation balue of $61.75 \mathrm{GeV}$;

- pass the list of L2jet candidates to L2GBL.

Table XL lists the parameters per jet object passed to L2GBL. 


\begin{tabular}{|c|c|}
\hline variable & Bits \\
\hline$\eta$ & 8 \\
$\phi$ & 8 \\
$E_{T}$ & 16 \\
$\eta_{\text {center }}$ & 8 \\
$\phi_{\text {center }}$ & 8 \\
$\eta_{\text {leadingTT }}$ & 8 \\
$\phi_{\text {leadingTT }}$ & 8 \\
Saturated EM tower & 1 \\
Saturated Total tower & 1 \\
$E_{T}$ is zero & 1 \\
\hline
\end{tabular}

TABLE XL JET OUTPUT TO L2GBL. 


\section{Appendix D}

\section{LEVEL-2 ELECTROMAGNETIC FILTERS AND TOOLS}

The L2 trigger decisions are formed in L2GBL. The trigger list for L2 uses scripts parsed to L2GBL. There is one script for every L1 trigger bit. Each script has a list of filters and the number of times that each filter has to pass the selection criteria. Each filter usually calls one tool, but filters can also call multiple tools or even other filters. The tools generate a list of objects that satisfy some basis criteria. The filters examine whether the objects provided by the tools pass the selection criteria determined by the trigger list.

The following are the parameters of a L2 EM filter (103):

- MAXEM - maximum number of EM objects allowed in filter;

- EMFRAC - EM fraction;

- ISOFRAC - isolation fraction;

- MINET - minimum transverse energy of the EM object;

- TOOL - the name of the EM tool used by the filter.

The objects satisfying the conditions of the EM filter can be passed into another filter, e.g. the invariant mass filter. The following are the conditions of an EM Tool (103):

- MAXEM - maximum number of EM objects allowed in the tool;

- REQUIRECPS - require match in the central preshower detector; 
- CPSWINDOWIETA - $\Delta \eta$ window for matching the EM object and a CPS cluster;

- CPSWINDOWIPHI - $\Delta \phi$ window for matching the EM object and a CPS cluster;

- REQUIRETRACK - require track matching with the EM object;

- TRACKFILTER - identify the type of tracks to use (e.g. CTT, STT, STT-IP);

- TRACKWINDOWIPHI - $\Delta \phi$ window for matching the EM object and a track;

- MINET - minimum transverse energy of the EM object;

- MINNEIGHBORET - minimum $\mathrm{E}_{T}$ value of the NN for the EM object to stay clustered;

- MINNEIGHBORETACENET - minimum $\mathrm{E}_{T}$ value of the NN in $\eta$ for CC;

- MINNEIGHBORPHICENET - minimum $\mathrm{E}_{T}$ value of the NN in $\phi$ for CC;

- MINNEIGHBORETAFWDET - minimum $\mathrm{E}_{T}$ value of the NN in $\eta$ for EC;

- MINNEIGHBORPHIFWDET - minimum $\mathrm{E}_{T}$ value of the NN in $\phi$ for EC;

- MINSINGLETOWEREMFRAC - minimum EM fraction to separate EM cluster into two single-trigger-tower EM objects;

- MINSINGLETOWERET - minimum transverve energy of a single-trigger-tower EM object. 


\section{Appendix E}

\section{SYSTEM8}

The System8 method is used to measure the b-tagging efficiency purely from data. It allows for the determination of selection efficiencies for signal and one or more backgrounds by measuring the inclusive selection efficiencies for one or more samples with varying signal and background compositions. The problem can be represented by a system of equations. The system is solvable if:

- the samples have different fractions of signal and backgrounds;

- the selection criteria have different efficiencies for these signal and backgrounds;

- the different selection criteria are decorrelated to allow the factorization of the efficiencies;

- the number of constraints $\geq$ the number of unknowns.

Two data samples are used for the measurement of the $b$-tagging efficiency, the muon-in-jet $(n)$ and the muon-in-jet away-jet-tagged sample $(p)$. The signal is given by $b$ jets, and only one background is considered, non- $b$ jets ( $c$ and light-quark jets). The two selection criteria used are the JLIP $b$ tagging and the SLT $b$ tagging, that requires the presence of a muon with $\Delta R(\mu, j e t)<0.5$ and $p_{T}^{r e l}>0.7 \mathrm{GeV}$, where $p_{T}^{\text {rel }}$ refers to the fraction of the muon momentum transverse to the momentum of the jet-muon system. A system of eight equations can be formed: 


\section{Appendix E (Continued)}

\begin{tabular}{|c|c|c|c|}
\hline$n$ & $=$ & $n_{b}+$ & $n_{n o n-b}$ \\
\hline$p$ & $=$ & $p_{b}+$ & $p_{\text {non-b}}$ \\
\hline$n^{J L I P}$ & $=\varepsilon_{b}^{J L I P}$ & $n_{b}+\varepsilon_{\text {non-b }}^{J L I P}$ & $n_{\text {non-b}}$ \\
\hline$p^{J L I P}$ & $=\varepsilon_{b}^{J L I P}$ & $p_{b}+\varepsilon_{n o n-b}^{J L I P}$ & $p_{n o n-b}$ \\
\hline$n^{S L T}$ & $=$ & $\varepsilon_{b}^{S L T} n_{b}+$ & $\varepsilon_{n o n-b}^{S L T} n_{n o n-b}$ \\
\hline$p^{S L T}$ & $=\beta$ & $\varepsilon_{b}^{S L T} p_{b}+\alpha$ & $\varepsilon_{\text {non-b }}^{S L T} p_{\text {non-b }}$ \\
\hline$n^{J L I P, S L T}$ & $=\varepsilon_{b}^{J L I P}$ & $\varepsilon_{b}^{S L T} n_{b}+\varepsilon_{n o n-b}^{J L I P}$ & $\varepsilon_{\text {non-b}}^{S L T} n_{n o n-b}$ \\
\hline$p^{J L I P, S L T}$ & $=\beta \varepsilon_{b}^{J L}$ & ${ }_{=}^{S L T} p_{b}+\alpha \varepsilon_{n o n}^{J L}$ & ${ }_{n-b}^{I P} \varepsilon_{\text {non-b}}^{S L T} p_{n o n-b}$ \\
\hline
\end{tabular}

The terms on the left hand side of the equations are measured in data and correspond to the total number of jets in each sample before tagging ( $n$ for the muon-in-jet sample and $p$ for the muon-in-jet away-jet-tagged sample) and after applying the corresponding tagger $\left(n^{J L I P}\right.$, $n^{S L T}, n^{J L I P, S L T}, p^{J L I P}, p^{S L T}$ and $\left.p^{J L I P, S L T}\right)$. The eight unknowns on the right hand side of the equations consist of the number of $b$ and non- $b$ jets in the two samples $\left(n_{b}, n_{n o n-b}, p_{b}\right.$, $\left.p_{n o n-b}\right)$, and the tagging efficiencies for taggable $b$ and non- $b$ jets for the two tagging algorithms JLIP and SLT. The system is solved for each $\left(p_{T}, \eta\right)$ bin separately. 
The $b$-tagging efficiency measurement is affected by uncertainties due to the assumption of the decorrelation between the JLIP and the SLT algorithm for $b$ jets

$$
\kappa_{b}=\frac{\varepsilon_{b}^{J L I P, S L T}}{\varepsilon_{b}^{J L I P} \varepsilon_{b}^{S L T}}
$$

for non- $b$-jets

$$
\kappa_{c l}=\frac{\varepsilon_{n o n-b}^{J L I P, S L T}}{\varepsilon_{n o n-b}^{J L I P} \varepsilon_{n o n-b}^{S L T T}}
$$

the sample dependence of the JLIP tagging efficiency for $b$ jets

$$
\beta=\frac{\varepsilon_{b}^{J L I P} \text { measured in the muon-in-jet away-jet-tagged sample }}{\varepsilon_{b}^{J L I P} \text { measured in the muon-in-jet sample }},
$$

and for non- $b$ jets

$$
\alpha=\frac{\varepsilon_{\text {non-b }}^{J L I P} \text { measured in the muon-in-jet away-jet-tagged sample }}{\varepsilon_{\text {non-b}}^{J L I P} \text { measured in the muon-in-jet sample }},
$$

particularly originating from the assumption that the non- $b$ background composition (i.e. the fraction of $c$ and light jets) of the two samples is the same. 


\section{Appendix F}

\section{BAYESIAN LIMIT CALCULATION}

The 95\% Confidence Level upper bound on the cross section is determined (104) by numerically solving the equation:

$$
\int_{0}^{\sigma^{95}} P(\sigma \mid N) d \sigma=0.95
$$

$\mathrm{P}(\sigma \mid \mathrm{N})$ is the probability that the cross section of a given value, $\sigma$ (in this analysis $\sigma$ is

$\sigma(\mathrm{ZH}) \times \mathrm{Br}(\mathrm{H} \rightarrow b \bar{b})$, is true, given the number of total events in data $(\mathrm{N})$ is true, after integrating out the luminosity $(\mathrm{L})$, the efficiency to pass all selection cuts $(\varepsilon)$, and total background (B):

$$
P(\sigma \mid N)=\int_{0}^{\infty} d L \int_{0}^{1} d \varepsilon \int_{0}^{\infty} d B P(N \mid \sigma, L, \varepsilon ; B) P(\sigma) P(L) P(\varepsilon) P(B)
$$

The total efficiency, $\varepsilon$, is the number of events passing all selection cuts divide by the initial generated events times the branching ratio of $Z \rightarrow e^{+} e^{-}$.

The probability distributions of the measured luminosity, the measured total efficiency, and the total MC simulated background processes are given by Gaussian distributions.

$$
\begin{aligned}
& P(L)=\frac{1}{\sqrt{2 \pi} \sigma_{L_{o}}} \varepsilon^{-\frac{\left(L-L_{o}\right)^{2}}{2 \sigma_{L_{o}}^{2}}} \\
& P(\varepsilon)=\frac{1}{\sqrt{2 \pi} \sigma_{\varepsilon_{o}}} e^{-\frac{\left(\varepsilon-\varepsilon_{o}\right)^{2}}{2 \sigma_{\varepsilon_{o}}^{2}}}
\end{aligned}
$$




\section{Appendix F (Continued)}

$$
P(B)=\frac{1}{\sqrt{2 \pi} \sigma_{B_{o}}} e^{-\frac{\left(B-B_{o}\right)^{2}}{2 \sigma_{B_{o}}^{2}}}
$$

$L_{o}, \varepsilon_{o}, B_{o}$ are the luminosity measurement, the signal efficiency times the branching ratio of $Z \rightarrow e^{+} e^{-}$, and the total number of background events. The three measurements and their corresponding uncertainties $\left(\sigma_{L_{o}}, \sigma_{\varepsilon_{o}}, \sigma_{B_{o}}\right)$ are passed to the Bayesian limit calculator.

$\mathrm{P}(\sigma)$ is chosen as $1 / \sigma_{\max }$ if $0 \leq \sigma \leq \sigma_{\max }$, otherwise $\mathrm{P}(\sigma)$ is zero. $\sigma_{\max }$ is an arbitrary large allowed maximum cross section. This choice for the cross section incorporates an absence of knowledge about the cross section. Since the probability of the cross section has no functional dependence (constant) on the probability equation, it is ignored (set to one).

$\mathrm{P}(\mathrm{N} \mid \sigma, \mathrm{L}, \varepsilon ; \mathrm{B})$ is the probability that the number of events measured in data, $\mathrm{N}$, is true given the signal MC cross section, luminosity, total efficiency, and total MC simulated background is true. $\mathrm{P}(\mathrm{N} \mid \sigma, \mathrm{L}, \varepsilon ; \mathrm{B})$ is modeled by a Poisson distribution:

$$
P(N \mid \sigma, L, \varepsilon ; B)=\frac{e^{-(L \varepsilon \sigma+B)}(L \varepsilon \sigma+B)^{N}}{N !}
$$

$\mathrm{L} \varepsilon \sigma+\mathrm{B}$ is the total amount of expected signal event plus the total amount of background events.

Bayes' Theorem relates what one knows about parameters (prior probabilities) before performing an experiment, to knowledge of the parameters (post probabilities) after performing the experiment. 
Bayes' Theorem is:

$$
P(\text { model } \mid \text { data })=\frac{P(\text { data } \mid \text { model }) P(\text { model })}{P(\text { data })}
$$

$\mathrm{P}$ (model) is $\mathrm{P}$ (signal and background), the probability of the number of signal and background events are true, or $\mathrm{P}(\sigma, L, \varepsilon ; \mathrm{B}) . \sigma, L, \varepsilon$, and $\mathrm{B}$ are assumed to be uncorrelated giving $\mathrm{P}(\sigma, L, \varepsilon ; \mathrm{B})=\mathrm{P}(\sigma) \mathrm{P}(L) \mathrm{P}(\varepsilon) \mathrm{P}(\mathrm{B})$. Therefore, Equation F.2 can be rewritten as (dropping the integrals):

$$
P(\sigma, L, \varepsilon ; B \mid N)=P(N \mid \sigma, L, \varepsilon ; B) P(\sigma, L, \varepsilon ; B) .
$$




\section{Appendix G}

\section{EVENT DISPLAYS}

The following event displays (Figure 119 to Figure 123) show the five events in data which passed the double b-tag selection cut. The details of the electron and jet objects are given. The event display on the left of each figure shows the calorimeter transverse energy deposits in a $\eta$ - $\phi$ view. The event display on the right of each figure shows the tracks and calorimeter transverse energy deposits in a $r$ - $\phi$ view. 

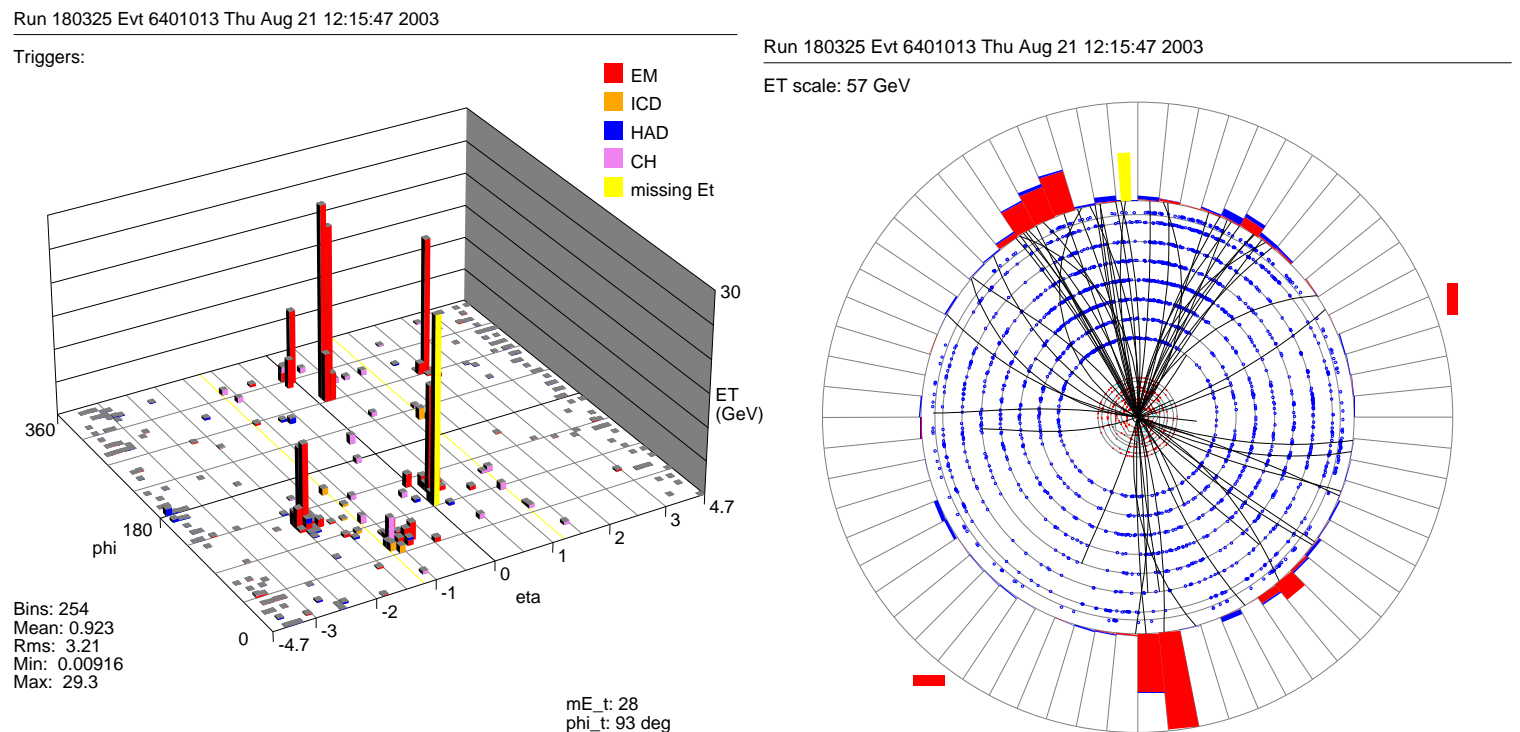

Figure 119. First of the five double b-tagged events.

Di-electron Inv. Mass=100.7; Double b-jet Inv. Mass=59.3;

Electron1: $p_{T}=74.2, \eta_{\text {detector }}=0.20, \phi_{\text {detector }}=4.82$, no track match;

Electron2: $p_{T}=30.4, \eta_{\text {detector }}=1.94, \phi_{\text {detector }}=4.77$, track $p_{T}=33.01 ;$ Jet1: $p_{T}=54.9$, $\eta_{\text {detector }}=0.17, \phi_{\text {detector }}=1.99$, JLIP prob. $=0.001$

Jet2: $p_{T}=49.8, \eta_{\text {detector }}=-1.92, \phi_{\text {detector }}=2.14$, not taggable;

Jet3: $p_{T}=33.6, \eta_{\text {detector }}=-0.91, \phi_{\text {detector }}=1.16, \mathrm{JLIP}$ prob. $=0.006 ; E_{T}^{\text {miss }}=18.4$, $E_{T}^{\text {miss }} \phi=1.67, P V_{Z}=17.1$ 


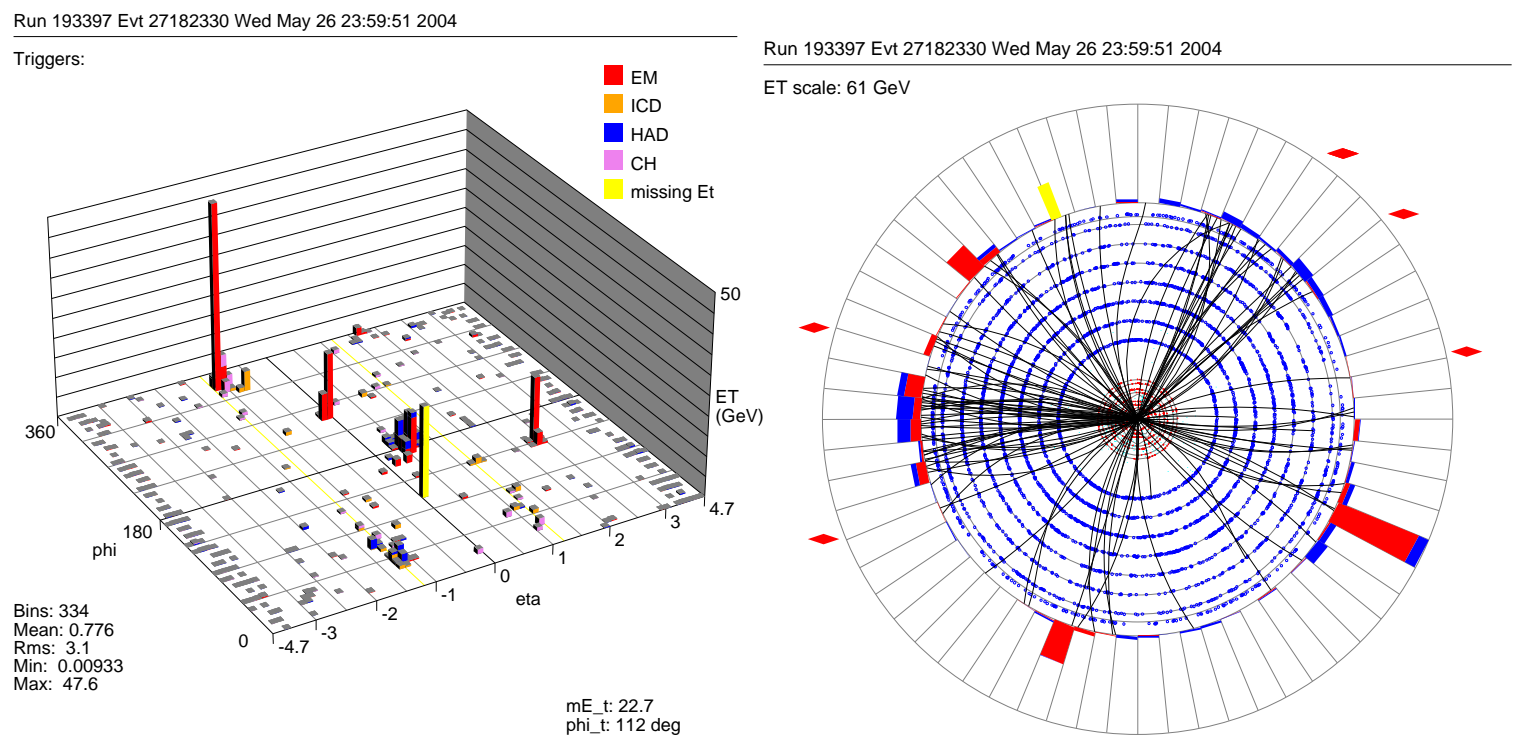

Figure 120. Second of the five double b-tagged events.

Di-electron Inv. Mass=87.0; Double b-jet Inv. Mass=165.4;

Electron1: $p_{T}=29.3, \eta_{\text {detector }}=-0.08, \phi_{\text {detector }}=4.40$, track $p_{T}=20.4$;

Electron2: $p_{T}=22.8, \eta_{\text {detector }}=2.37, \phi_{\text {detector }}=2.37$, track $p_{T}=4.88$;

Jet1: $p_{T}=120.0, \eta_{\text {detector }}=-1.09, \phi_{\text {detector }}=5.99$, not taggable;

Jet2: $p_{T}=86.9, \eta_{\text {detector }}=0.50, \phi_{\text {detector }}=3.23$, JLIP prob. $=0.001$;

Jet3: $p_{T}=41.1, \eta_{\text {detector }}=-1.18, \phi_{\text {detector }}=0.91$, JLIP prob. $=0.001$;

$E_{T}^{m i s s}=21.7, E_{T}^{\text {miss }} \phi=2.15, P V_{Z}=-25.6$ 


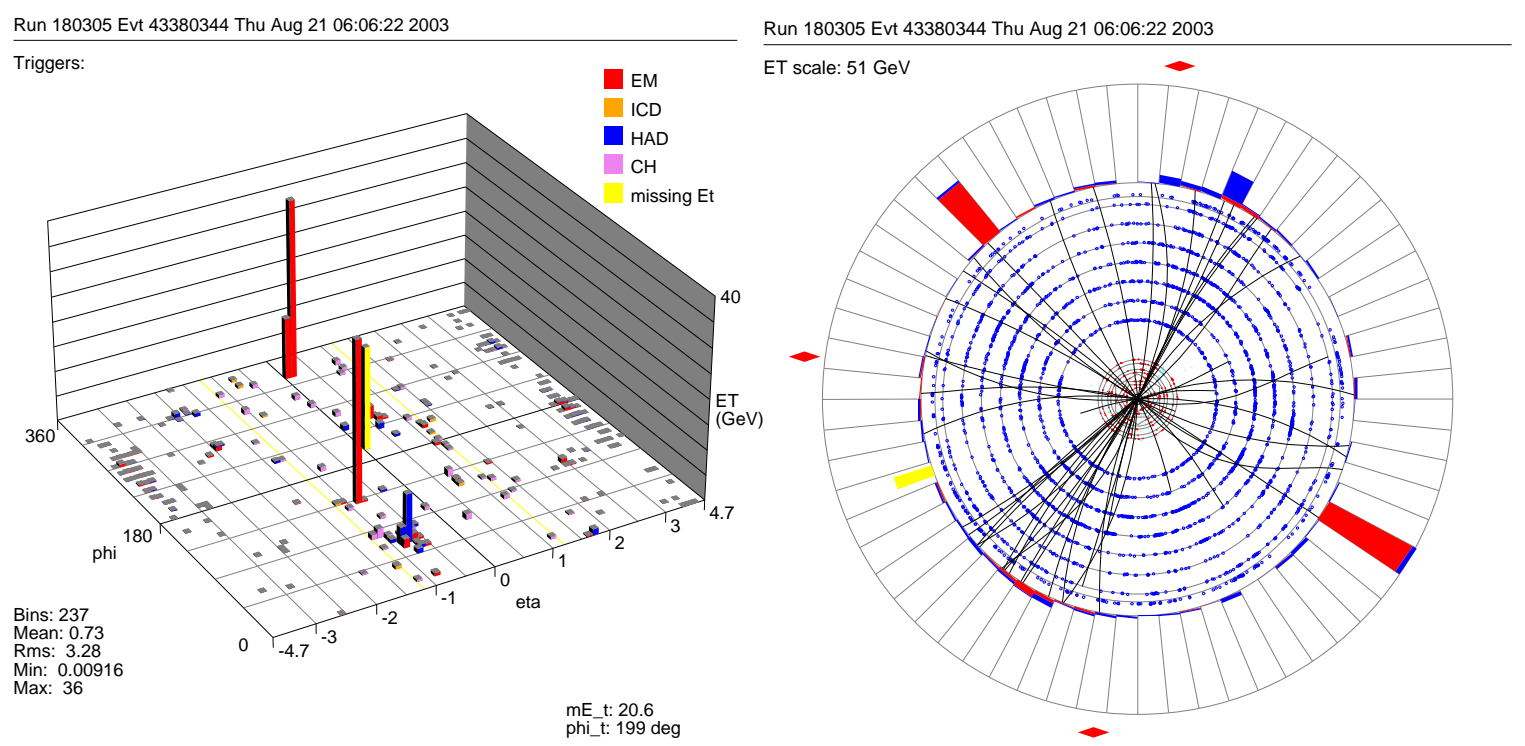

Figure 121. Third of the five double b-tagged events.

Di-electron Inv. Mass $=88.7$; Double b-jet Inv. Mass $=83.8$

Electron1: $p_{T}=53.2, \eta_{\text {detector }}=0.10, \phi_{\text {detector }}=5.72$, track $p_{T}=35.5$;

Electron2: $p_{T}=31.6, \eta_{\text {detector }}=-0.83, \phi_{\text {detector }}=2.31$, track $p_{T}=23.9$;

Jet1: $p_{T}=43.3, \eta_{\text {detector }}=-0.72, \phi_{\text {detector }}=1.21$, JLIP prob. $=0.002$;

Jet2: $p_{T}=28.9, \eta_{\text {detector }}=0.48, \phi_{\text {detector }}=4.25$, JLIP prob. $=0.026$;

$$
E_{T}^{m i s s}=10.4, E_{T}^{m i s s} \phi=3.01, P V_{Z}=29.9
$$




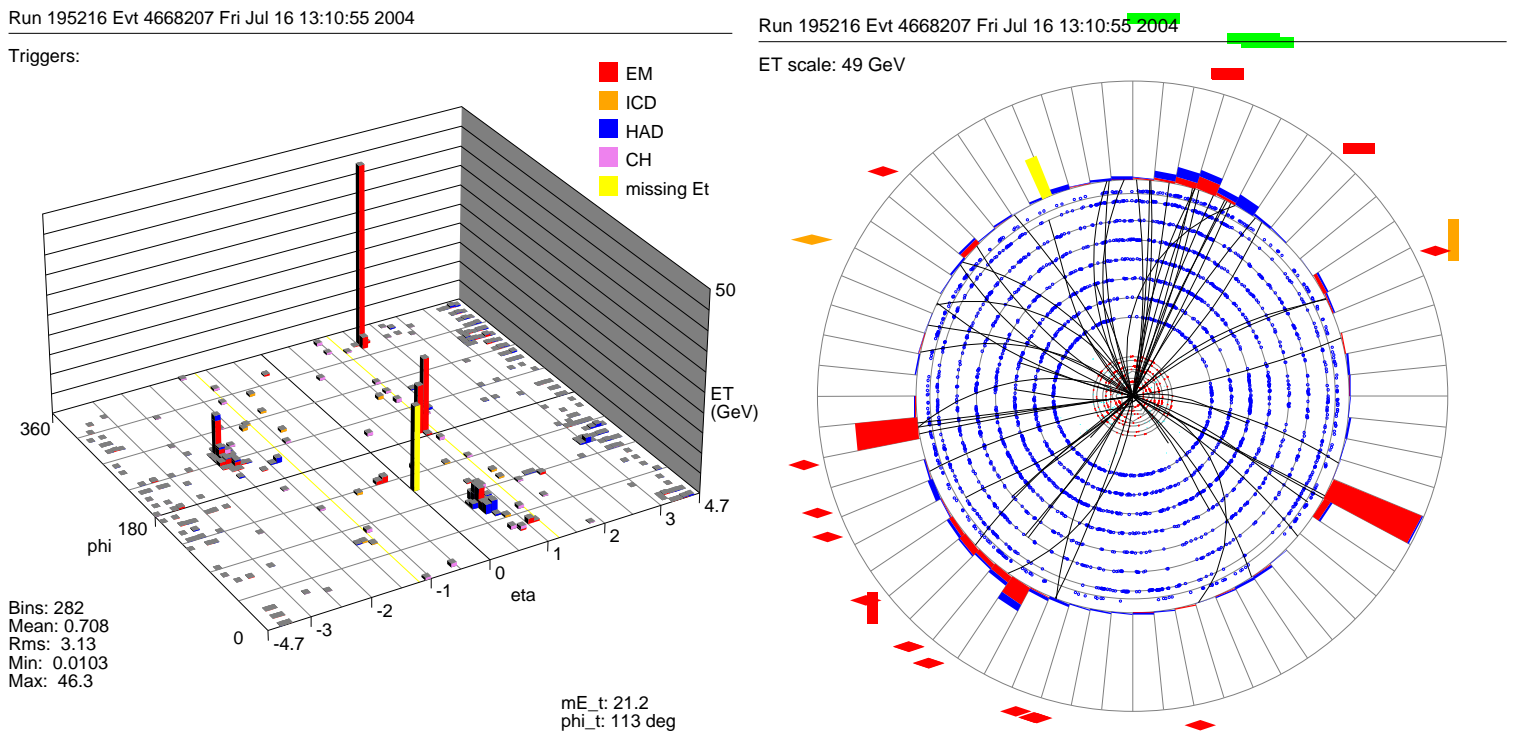

Figure 122. Four of the five double b-tagged events.

Di-electron Inv. Mass=85.3; Double b-jet Inv. Mass $=223$;

Electron1: $p_{T}=52.5, \eta_{\text {detector }}=1.55, \phi_{\text {detector }}=5.81$, tracks $p_{T}=34.4$;

Electron2: $p_{T}=34.8, \eta_{\text {detector }}=1.00, \phi_{\text {detector }}=3.28$, track $p_{T}=26.3$;

Jet1: $p_{T}=63.0, \eta_{\text {detector }}=0.69, \phi_{\text {detector }}=1.29$, JLIP prob. $=0.006$;

Jet2: $p_{T}=44.8, \eta_{\text {detector }}=-2.01, \phi_{\text {detector }}=4.17$, JLIP prob. $=0.036$;

$E_{T}^{m i s s}=14.7, E_{T}^{\text {miss }} \phi=2.07, P V_{Z}=-3.2$ 


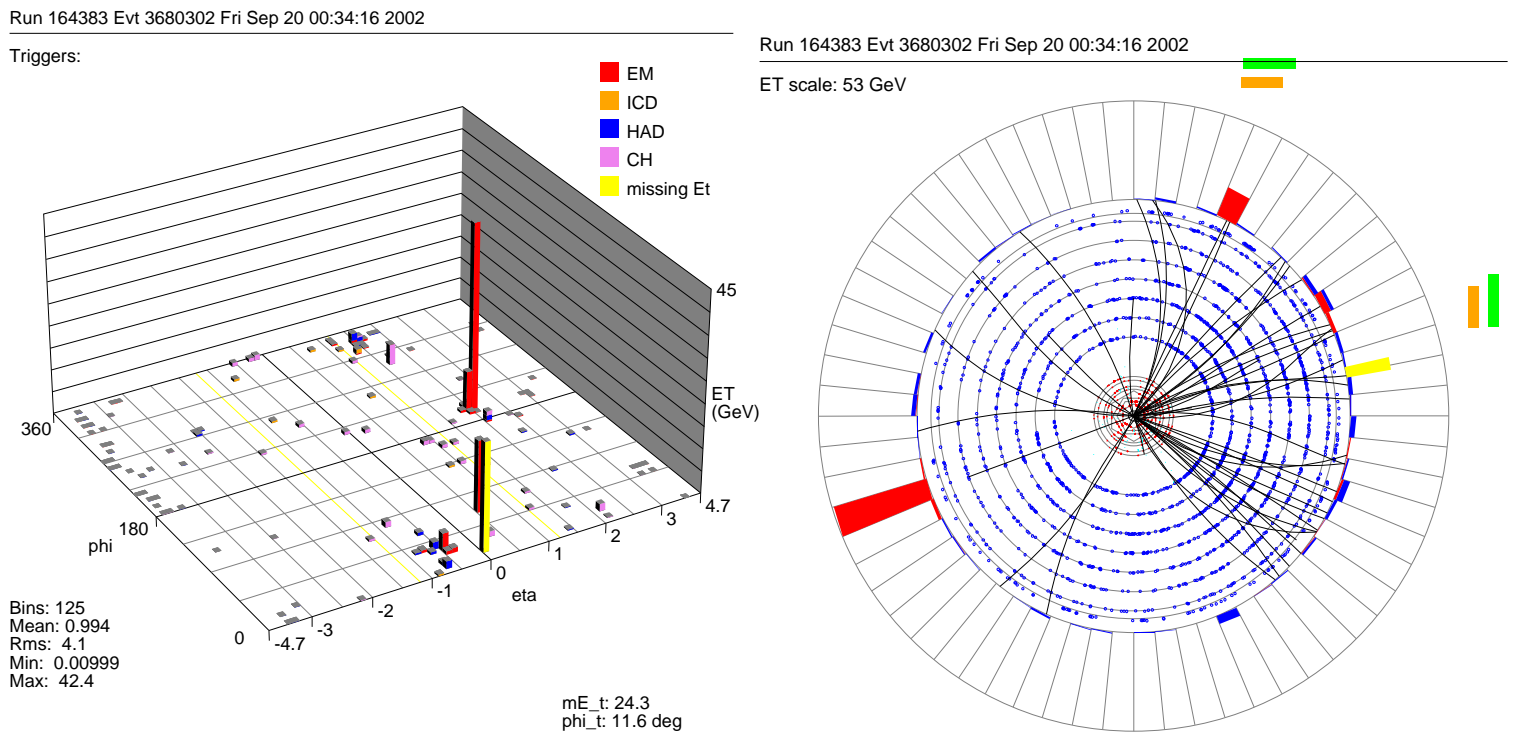

Figure 123. Fifth of the five double b-tagged events.

Di-electron Inv. Mass $=89.6$; Double b-jet Inv. Mass $=65.4$;

Electron1: $p_{T}=62.7, \eta_{\text {detector }}=2.02, \phi_{\text {detector }}=3.49$, track $p_{T}=433$;

Electron2: $p_{T}=20.0, \eta_{\text {detector }}=0.55, \phi_{\text {detector }}=1.13$, track $p_{T}=19.2$;

Jet1: $p_{T}=26.2, \eta_{\text {detector }}=-0.52, \phi_{\text {detector }}=0.61$, JLIP prob. $=0.020$;

Jet2: $p_{T}=20.7, \eta_{\text {detector }}=1.51, \phi_{\text {detector }}=6.03$, JLIP prob. $=0.026$;

$E_{T}^{\text {miss }}=18.6, E_{T}^{\text {miss }} \phi=0.96, P V_{Z}=12.4$ 


\section{CITED LITERATURE}

1. Griffiths, D.: Introduction to Elementary Particles., John Wiley \& Sons, 1987.

2. Quigg, C.: Gauge Theories of the Strong, Weak, and Electromagnetic Interactions. Westview Press, 1983.

3. Gell-Man, M.: Phys. Lett., 8 214, 1964.

4. Zweig, G.: CERN Report 8182/TH., 401, 1964.

5. Pennington, M.: Rep. Prog. Phys., 46 393, 1983.

6. Muta, T.: Foundations of Quantum Chromodynamics. World Scientific Publishing Co. 1987.

7. Fritzsch H., et al.: Phys. Lett., 47B 365, 1973.

8. Fritzsh H., et al.: Phys. Lett., 47B 365, 1973.

9. Gross, D.: Phys. Rev. Lett., 30 1343, 1973.

10. Politzer, H.: Phys. Rev Lett., 30 1346, 1973.

11. Field, R.: Applications of Perturbative QCD. Addison-Wesley Publishing Co., 1989.

12. Yao, W. et al.: Journal of Physics, G 33 1, 2006.

13. Higgs, P.: Broken Symmetries, Massless Particles and Gauge Fields. Phys. Lett., 12 132, 1964.

14. Higgs, P.: Broken Symmetries and the Masses of Gauge Bosons. Phys. Lett., 13 508, 1964.

15. Higgs, P.: Spontaneous Symmetry Breaking without Massless Bosons. Phys. Rev., 145 1156, 1966. 
16. The LEP Electroweak Working Group. 2006. http://lepewwg.web.cern.ch/LEPEWWG/.

17. Abbiendi, G. et al: Phys. Lett. B., 565 61, 2003.

18. Spira, M.: Higgs boson production and decay at the Tevatron. hep-ph/9810289. 1998.

19. Fermi National Accelerator Laboratory. www.fnal.gov.

20. Thompson, J: Introduction to Colliding Beams at Fermilab. FERMILAB-TM-1909. 1994.

21. Accelerator Concepts Rookie Books. 2003. http://www-bdnew.fnal.gov/operations/rookie_books/rbooks.html.

22. Abazov, V. et al: The Upgraded DØ Detector. Fermilab-Pub-05/341-E, 2005.

23. Edwards, T. et al.: Determination of the Effective Inelastic $p \bar{p}$ Cross Section for the DØ Run II Luminosity Measurement. FERMILAB-TM-2278-E, 2004.

24. DØ Collaboration: DØ Silicon Tracker Technical Design Report. 1994. http://d0server1.fnal.gov/projects/silicon/www/tdr_final.ps.

25. DØ Collaboration: The DØ Upgrade: Central Tracker Technical Design Report. 1999. http://d0server1.fnal.gov/users/stefan/www/CFT_TDR/CFT_TDR.ps.

26. Adams, M. et al.: Design Report of the Central Preshower Detector for the DØ Upgrade. http://d0server1.fnal.gov/users/qianj/CPS/doc/dn3104.pdf.

27. Gordeev, A. et al.: Technical Design Report of the Forward Preshower Detector for the DØ Upgrade. DØ Note 3445, 1998.

28. Ferbel, T. : Experimental Techniques in High Energy Physics. Addison-Wesley, 1987.

29. Baldin, B. et al.: Technical Design Report of the Central Muon System. DØ Note 3365, 1997.

30. Alexeev, G. et al.: Technical Design Report of the DØ Forward Muon Tracking Detector Based on Mini-Drift Tubes. DØ Note 3366, 1997. 
31. Blazey, J.: The DØ Run II Trigger.

http://niuhep.physics.niu.edu/ blazey/rt.ps.

32. Abolins, M. et al.: DØ Run II Level 1 Trigger Framework Technical Design Report. 1998. http://www.pa.msu.edu/hep/d0/ftp/11/framework/11fw_tdr_05june98.txt.

33. Linnemann, J.: D $\varnothing$ Run II Level 2 Trigger. http://www.pa.msu.edu/hep/d0/12/index.html.

34. Edmunds, D. et al.: Technical Design Report for the Level 2 Global Processor. 1998. www.pa.msu.edu/hep/d0/ftp/l2/overview/globaltdr/global_tdr.ps.

35. Boehnlien, A. et al.: Decription of the DØ L3 Trigger software components. DØ Note 3630, 1999.

36. Snyder, S.: COOR. 2006.

http://www-d0.fnal.gov/d0dist/dist/packages/coor/devel/doc/coorover.ps.

37. Angstadt, R. et al.: The DØ L3 Trigger Data Acquisition System. IEEE Transactions on Nuclear Science, Volume 51 Issue 3, 2004.

38. VITA: VME Technology.

http://www.vita.com/vmefaq.html.

39. Cisco Catalyst 6509 Switch.

http://www.cisco.com/.

40. DØ Collaboration: DØ Reco. 2004.

http://www-d0.fnal.gov/computing/algorithms/status/p14.html.

41. Khanov, A.: HTF: Histogramming Method for Finding Tracks. DØ Note 3778, 2000.

42. Borisov, G.: Technical Details of AA Tracking. 2004.

www-d0.fnal.gov/global_tracking/talks/20030228/talk-adm-030228.ps.

43. Greenlee, H.: The DØ Kalman Track Fit. DØ Note 4303, 2004.

44. Garcia-Bellido, A. et al.: Primary Vertex certification p14. DØ Note 4320, 2004. 
45. Schwartzman, A. and Narain, M.: Probabilistic Primary Vertex Selection. DØ Note 4042, 2002.

46. Fleuret, F.: The DØ Electron/Photon Analysis Package EMAnalyze. 2001. http://www-d0.fnal.gov/d0dist/dist/releases/p14.06.01/em_analyze/doc/ EMAnalyze_doc.ps.

47. Blazey, J. et al.: Run II Jet Physics. hep-ex/0005012, 2000.

48. Andrieu, B. and Busato, E.:Jet Algorithms in the DØ Run II Software: Description and User's Guide. DØ Note 4457, 2004.

49. Agram, J.-L. et al.: Jet Energy Scale at DØ Run II. DØ Note 4720, 2005.

50. Block, D. and Clement, B.: Jet LIfetime Probability b-tag Certification. http://www-d0.fnal.gov/phys_id/bid/d0_private/certification/p14Pass2/JLIP/ Jetprob_v1.html.

51. Bloch, D. et al: Performance of the JLIP b-tagger in p14. DØ Note 4348, 2004.

52. Bloch, D. and Clement, B.: Update of the JLIP b-tagger Performance in p14/pass2 with JES 5.3. DØ Note 4824, 2005.

53. Feligioni, L. et al: Taggability in Data and Monte Carlo. http://www-d0.fnal.gov/phys_id/bid/d0_private/certification/p14/secvertex/ secvertex_v1.html\#tagga.

54. Sjoestrand, T. et al.: PYTHIA 6.3 Physics and Manual. hep-ph/0308153, 2003.

55. Mangan, M.: ALPGEN V1.3. http://mlm.home.cern.ch/mlm/alpgen/.

56. Campbell, J. and Ellis, K.: MCFM - Monte Carlo for FeMtobarn processes. 2006. http://mcfm.fnal.gov/.

57. CTEQ: CTEQ5 Parton Distributions. Eur. Phys. J., C12:375-392, 2000.

58. Altarelli, G. and Parisi, G.: Asymptotic freedom in parton language. Nucl. Phys. B, 126:298, 1977. 
59. Gribov, V. and Lipatov, L.: Sov. J. Nucl. Phys., 15:438, 1972.

60. Dokshitzer, Y.: Sov. J. Phys. JETP, 46:641, 1977.

61. Fisyak, Y. and Womersley, J.: DØ GEANT Simulation of the Total Apparatus Response. DØ Note 3191, 1997.

62. CERN: GEANT - Detector Description and Simulation Tool. 2003. http://wwwasd.web.cern.ch/wwwasd/geant/.

63. Eno, S. et al.: DØ Sim. 2001. http://www-d0.fnal.gov/computing/MonteCarlo/simulation/d0sim.html.

64. Bassler, U. et al.: Technical Description of the T42 Algorithm for the Calorimeter Noise Suppression.DØ Note 4146, 2003.

65. Suyong, C.: 2003

http://www-d0.fnal.gov/ suyong/d0_private/athenaweb/athena.htm.

66. Duflot, L. et al.: cal_event_quality package. DØ Note 4514, 2004.

67. Stone, A.: 2005. http://nippon.fnal.gov/ alstone/D0Work/Athena/badruns/badruns.html.

68. Stone, A.: 2005. http://nippon.fnal.gov/ alstone/D0Work/Athena/lumi/lumi.html.

69. Luminosity ID group: 2005. http://www-d0.fnal.gov/phys_id/luminosity/data_access.

70. Snow, G.: Adjustments to the Meaured Integrated Luminosity for RunIIa. DØ Note 5139, 2006.

71. Jain, S.: Scale and Over-smearing for MC Electron. DØ Note 4402, 2004.

72. Agelou, M. et al: DØ Top Analysis and Data Sample for the Winter Conference. DØ Note $\underline{4419}, 2004$.

73. Campbell, J. et al.: Production of a $\mathrm{Z}$ boson and two jets with one heavy-quark tag. hep-ph/0510362, 2005. 
74. Heinmiller, J. et al.: Jet Reconstruction Efficiency. DØ Note 4837, 2005.

75. Grivez, J. and Makovec, N.: Shifting, Smearing, and Removing Simulated Jets. DØ Note 4914, 2005.

76. Ciccolini, M. et al.: Electroweak Radiative Corrections to Associated WH and ZH Production at Hadron Colliders. hep-ph/0306234, 2003.

77. Bonciani, R. et al.: Nucl. Physics. B, 529 424, 1998.

78. Cacciari, M. et al.: JHEP, 0404 068, 2004.

79. Kidonakis, N. and Vogt, R.: Phys. Rev. D, 68 114014, 2003.

80. Nunnemann, T.: MCFM Cross Sections: 2004. http://www-clued0.fnal.gov/ nunne/cross-sections/mcfm_cross-sections.html.

81. Bloch, D. et al.: SystemD or how to get signal, backgrounds, and their efficiencies with real data. DØ Note 4159, 2003.

82. Bertram, I. et al.: Fermilab-TM-2104, 2000.

83. Yao, W.-M. et al.: Journal of Physics G, 33 1, 2006.

84. D. Acosta et al., The CDF Collaboration: Search for Higgs Bosons Decaying into $b \bar{b}$ and Produced in Association with a Vector Boson in $p \bar{p}$ Collisions at $\sqrt{s}=1.8 \mathrm{TeV}$. Phys. Rev. Lett. 95 051801, 2005.

85. Gregorio, B.: Search for the SM Higgs boson at the Tevatron. ICHEP, 2006.

86. Yen, C.-S.: G-link: A Chipset for Gigabit-Rate Data Communication. Hewlett-Packard Journal, 1992.

87. Electronics Systems Engineering: VRB Transition Module. http://www-ese.fnal.gov/SVX/Production/SVX_Web/VTM/VTM_Spec.pdf.

88. Bernard, R. et al: FIC Module Specifications. http://www-d0online.fnal.gov/www/groups/trigger/l2/cards/fic/doc/fic.pdf. 
89. Electronics Systems Engineering: VME Readout Buffer. http://www-ese.fnal.gov/eseproj/svx/vrb/vrb.htm.

90. Cypress Semiconductor Corporation.

http://www.cypress.com

91. Snow, G. et al: CIC and SFO. http://unlhep2.unl.edu/ GSNOW/CIC_SFO.html.

92. Baden, D. et al.: MBT Technical Design Report. http://galileo.phys.virginia.edu/ rjh2j/l2beta/alpha_docs/mbt-tdr-v6.ps.

93. Baden, D. et al.: DØ Level 2 Magic Bus.

http://galileo.phys.virginia.edu/ rjh2j/12beta/beta_docs/d0_magicbus.pdf.

94. Hirosky, B.: Level 2 beta Project.

http://galileo.phys.virginia.edu/ rjh2j/12beta/.

95. Pulse Research Lab.

http://www.pulseresearchlab.com/faqs/ecl_ques/ecl_Q1-Q4.htm\#Q3:

96. Edmunds, D. et al: Serial Command Link Description D-Zero Run II Trigger DAQ System. http://www.pa.msu.edu/hep/d0/ftp/scl/scl_description.pdf.

97. Chapin, D.: The DØ Level 3 Data Acquisition System.

http://www-d0.fnal.gov/runcoor/DAQ/Tutorials/2004/2004-05-

18_Chapin_L3DAQ.pdf.

98. Hewlett-Packard Development Company. http://h18002.www1.hp.com/alphaserver/technology/chipdocs.html?jumpid=reg_R1002_USEN.

99. Huang, Z. and Miller, S.: Level 2 Process Board Technical Reference Manual. 1998. http://www-d0online.fnal.gov/www/groups/trigger/12/online/uic_alpha/ 12alpha/alphaboard_technical.ps

100. International Engineering Consortium. http://www.iec.org/online/tutorials/compact_pci/index.html. 
101. Advantech.

http://www.advantech.com.

102. Adams, M. et al: Level-2 Calorimeter Preprocessor Technical Design Report. DØ Note $3651,1999$.

103. Casey, D.: Writing Filter and Tools for L2Global v0.5. 2000.

http://www-d0online.fnal.gov/www/groups/trigger/l2/global/WritingL2Filters.pdf.

104. Hobbs, J.: Simple Limit Calculator.

http://www-clued0.fnal.gov/ hobbs/limit_calc/limit_calc.html. 